quatrième série-tome 42 fascicule 3 mai-juin 2009

$$
\begin{aligned}
& \text { ANNALES } \\
& \text { SCIENTIFIQUES } \\
& \text { de } \\
& \text { L'ECOLE } \\
& \text { NORMALE } \\
& \text { SUPÉRIEURE }
\end{aligned}
$$

Francis C. S. BROWN

Multiple zeta values and periods of moduli spaces $\overline{\mathfrak{M}}_{0, n}$ 
Ann. Scient. Éc. Norm. Sup.

$4^{\text {e }}$ série, t. 42,2009 , p. 371 à 489

\title{
MULTIPLE ZETA VALUES AND PERIODS OF MODULI SPACES $\overline{\mathfrak{M}}_{0, n}$
}

\author{
BY FrancIS C. S. BROWN
}

Abstract. - We prove a conjecture due to Goncharov and Manin which states that the periods of the moduli spaces $\mathfrak{M}_{0, n}$ of Riemann spheres with $n$ marked points are multiple zeta values. We do this by introducing a differential algebra of multiple polylogarithms on $\mathfrak{M}_{0, n}$ and proving that it is closed under the operation of taking primitives. The main idea is to apply a version of Stokes' formula iteratively to reduce each period integral to multiple zeta values.

We also give a geometric interpretation of the double shuffle relations, by showing that they are two extreme cases of general product formulae for periods which arise by considering natural maps between moduli spaces.

RÉSUMÉ. - Nous démontrons une conjecture de Goncharov et Manin qui prédit que les périodes des espaces de modules $\mathfrak{M}_{0, n}$ des courbes de genre 0 avec $n$ points marqués sont des valeurs zêta multiples. Nous introduisons une algèbre différentielle de fonctions polylogarithmes multiples sur $\mathfrak{M}_{0, n}$ dans laquelle il existe des primitives. L'idée principale est d'appliquer une version de la formule de Stokes récursivement pour réduire chaque intégrale de périodes à une combinaison linéaire de valeurs zêta multiples.

Nous donnons également une interprétation géométrique des double relations de mélange pour les valeurs zêta multiples. En considérant des applications naturelles entre les espaces des modules, on déduit des formules de produit générales entre leurs périodes. Les doubles relations de mélange s'obtiennent comme deux cas particuliers de cette construction.

\section{Introduction}

Let $n=\ell+3 \geq 4$, and let $\mathfrak{M}_{0, n}$ denote the moduli space of curves of genus 0 with $n$ marked points. There is a smooth compactification $\overline{\mathfrak{M}}_{0, n}$, defined by Deligne, Knudsen and Mumford, such that the complement

$$
\overline{\mathfrak{M}}_{0, n} \backslash \mathfrak{M}_{0, n}
$$


is a normal crossing divisor. Let $A, B \subset \overline{\mathfrak{M}}_{0, n} \backslash \mathfrak{M}_{0, n}$ denote two sets of boundary divisors which share no irreducible components. In [27], Goncharov and Manin show that the relative cohomology group

$$
H^{\ell}\left(\overline{\mathfrak{M}}_{0, n} \backslash A, B \backslash B \cap A\right)
$$

defines a mixed Tate motive which is unramified over $\mathbb{Z}$.

On the other hand, let $n_{1}, \ldots, n_{r} \in \mathbb{N}$, and suppose that $n_{r} \geq 2$. The multiple zeta value $\zeta\left(n_{1}, \ldots, n_{r}\right)$ is the real number defined by the convergent sum

$$
\zeta\left(n_{1}, \ldots, n_{r}\right)=\sum_{0<k_{1}<\cdots<k_{r}} \frac{1}{k_{1}^{n_{1}} \ldots k_{r}^{n_{r}}} .
$$

Its weight is the quantity $n_{1}+\cdots+n_{r}$, and its depth is the number of indices $r$. We will say that the period $2 i \pi$ has weight 1 . A very general conjecture [25] claims that the periods of any mixed Tate motive unramified over $\mathbb{Z}$ are multiple zeta values. In the case of the motives (1.1) arising from moduli spaces, this says the following. Consider a real smooth compact submanifold $X_{B} \subset \overline{\mathfrak{M}}_{0, n}$ of dimension $\ell$, whose boundary is contained in $B$ and which does not meet $A$. It represents a class in $H_{\ell}\left(\overline{\mathfrak{M}}_{0, n}, B\right)$. Let $\omega_{A} \in \Omega^{\ell}\left(\overline{\mathfrak{M}}_{0, n} \backslash A\right)$ denote an algebraic form with singularities contained in $A$. In [27], Goncharov and Manin conjectured that the integral

$$
I=\int_{X_{B}} \omega_{A}
$$

is a linear combination of multiple zeta values, and proved that every multiple zeta value can occur as such a period integral. In this paper, we develop some general methods for computing periods and prove this conjecture as an application.

Theorem 1.1. - The integral I is a $\mathbb{Q}[2 \pi i]$-linear combination of multiple zeta values of weight at most $\ell$.

This theorem thus lends weight to the conjecture on the periods of all mixed Tate motives which are unramified over $\mathbb{Z}$.

The rough idea of our method is as follows. The set of real points $\mathfrak{M}_{0, n}(\mathbb{R})$ is tesselated by a number of open cells $X_{n}$ which can naturally be identified with a Stasheff polytope, or associahedron. First consider the case where the domain of integration in (1.3) is a single cell $X_{n}$ (this actually suffices for the version of the conjecture considered in [27]). The key is then to apply a version of Stokes' theorem to the closed polytope $\bar{X}_{n} \subset \overline{\mathfrak{M}}_{0, n}(\mathbb{R})$. Since each face of $\bar{X}_{n}$ is itself a product of associahedra $\bar{X}_{a} \times \bar{X}_{b}$, we repeatedly take primitives to obtain a cascade of integrals over associahedra of smaller and smaller dimension. In order to do this, we need to construct a graded algebra $L\left(\mathfrak{M}_{0, n}\right)$ of multiple polylogarithm functions on $\mathfrak{M}_{0, n}$ in which primitives exist. At each stage of the induction, the dimension of the domain of integration decreases by one, and the weight of the integrand increases by one. At the final stage, we evaluate a multiple polylogarithm at the point 1 , and this gives a linear combination of multiple zeta values. This gives an effective algorithm for computing such integrals. Our approach also works in greater generality, and our results should extend without difficulty, for example, to the case of configuration spaces related to other Coxeter groups. 


\subsection{General overview}

This paper is essentially a study of the de Rham theory of the motivic fundamental group of $\mathfrak{M}_{0, n}$. Previously, the focus has mainly been on the projective line minus roots of unity, and in particular $\mathfrak{M}_{0,4} \cong \mathbb{P}^{1} \backslash\{0,1, \infty\}$ ([13], [14], [25, 26], [45]). The advantage of considering the moduli spaces $\mathfrak{M}_{0, n}$ is that we can bring to bear the full richness of their geometry. We show, for example, that the double shuffle relations for multiple zeta values are just two special cases of generalised product relations arising naturally from functorial maps between moduli spaces.

An essential part of this work is devoted to multiple polylogarithms, which are functions first defined by Goncharov for all $n_{1}, \ldots, n_{\ell} \in \mathbb{N}$ by the power series:

$$
\operatorname{Li}_{n_{1}, \ldots, n_{\ell}}\left(x_{1}, \ldots, x_{\ell}\right)=\sum_{0<k_{1}<\cdots<k_{\ell}} \frac{x_{1}^{k_{1}} \ldots x_{\ell}^{k_{\ell}}}{k_{1}^{n_{1}} \ldots k_{\ell}^{n_{\ell}}}, \quad \text { where } \quad\left|x_{i}\right|<1 .
$$

By analytic continuation, they define multi-valued functions on $\mathfrak{M}_{0, n}$, where $n=\ell+3$. One of our main objects of study in this paper is the larger set $L\left(\mathfrak{M}_{0, n}\right)$ of all homotopy-invariant iterated integrals on $\mathfrak{M}_{0, n}$. It forms a differential algebra of multi-valued functions on $\mathfrak{M}_{0, n}$, in which the set of functions (1.4) is strictly contained. From the point of view of differential Galois theory, $L\left(\mathfrak{M}_{0, n}\right)$ defines a maximal unipotent Picard-Vessiot theory on $\mathfrak{M}_{0, n}$. We then define the universal algebra of multiple polylogarithms $B\left(\mathfrak{M}_{0, n}\right)$ to be a modified version of Chen's reduced bar construction. It is a differential graded Hopf algebra which is an abstract algebraic version of $L\left(\mathfrak{M}_{0, n}\right)$. One of our key results states that the de Rham cohomology of $B\left(\mathfrak{M}_{0, n}\right)$ is trivial. From this we deduce the existence of primitives in $L\left(\mathfrak{M}_{0, n}\right)$. We also need to understand the regularised restriction of polylogarithms to the faces of $\bar{X}_{n}$. This requires a canonical regularisation theorem, and amounts to studying what happens when singularities of an iterated integral collide. We are thus led to work on certain blow-ups of $\mathfrak{M}_{0, n}$, described below. It follows that the structure of $L\left(\mathfrak{M}_{0, n}\right)$, and hence the function theory of multiple polylogarithms, is intimately related to the combinatorics of the associahedron.

\subsection{Detailed summary of results}

In Section 2, we review some aspects of the geometry of the moduli spaces $\mathfrak{M}_{0, n}$, and study certain blow-ups obtained from them. Let $S$ denote a set with $n$ elements, each labelling a marked point on the projective line $\mathbb{P}^{1}$, and write $\mathfrak{M}_{0, S}=\mathfrak{M}_{0, n}$. A dihedral structure on $S$ is an identification of $S$ with the set of edges (or vertices) of an unoriented $n$-gon. For each such dihedral structure $\delta$, we embed $\mathfrak{M}_{0, S}$ in the affine space $\mathbb{A}^{\ell}$, where $\ell=n-3$, and blow up parts of the boundary in $\mathbb{A}^{\ell} \backslash \mathfrak{M}_{0, S}$ to obtain an intermediary space

$$
\mathfrak{M}_{0, S} \subset \mathfrak{M}_{0, S}^{\delta} \subset \overline{\mathfrak{M}}_{0, S},
$$

where $\mathfrak{M}_{0, S}^{\delta}$ is an affine scheme defined over $\mathbb{Z}$. We prove that the set of $\mathfrak{M}_{0, S}^{\delta}$, for varying $\delta$, forms a set of smooth affine charts on $\overline{\mathfrak{M}}_{0, S}$. In order to define them, we introduce dihedral coordinates, which are one of the key tools used throughout this paper. These are functions

$$
u_{i j}: \mathfrak{M}_{0, S} \rightarrow \mathbb{P}^{1} \backslash\{0,1, \infty\}, \quad \text { where } \quad\{i, j\} \in \chi_{S, \delta},
$$


indexed by the set of chords $\chi_{S, \delta}$ in the $n$-gon defined by $\delta$. Together, they define an embedding $\left(u_{i j}\right)_{\chi_{S, \delta}}: \mathfrak{M}_{0, S} \rightarrow \mathbb{A}^{n(n-3) / 2}$, and the scheme $\mathfrak{M}_{0, S}^{\delta}$ is the Zariski closure of the image of this map. For example, in the case $n=5$, we can identify $\mathfrak{M}_{0, S}=\left\{\left(t_{1}, t_{2}\right) \in \mathbb{P}^{1} \times \mathbb{P}^{1}\right.$ : $\left.t_{1} t_{2}\left(1-t_{1}\right)\left(1-t_{2}\right)\left(t_{1}-t_{2}\right) \neq 0, t_{1}, t_{2} \neq \infty\right\}$. The pentagon $(S, \delta)$ has five chords, labelled $\{13,24,35,41,52\}$ (fig. 1), and we have

$$
u_{13}=1-t_{1}, \quad u_{24}=\frac{t_{1}}{t_{2}}, \quad u_{35}=\frac{t_{2}-t_{1}}{t_{2}\left(1-t_{1}\right)}, \quad u_{41}=\frac{1-t_{2}}{1-t_{1}}, \quad u_{52}=t_{2} .
$$

The scheme $\mathfrak{M}_{0,5}^{\delta}$ is then defined by the five cyclically symmetric equations in $\mathbb{A}^{5}$ :

$$
u_{13}+u_{24} u_{52}=1, u_{24}+u_{35} u_{13}=1, \ldots, u_{52}+u_{13} u_{14}=1 .
$$

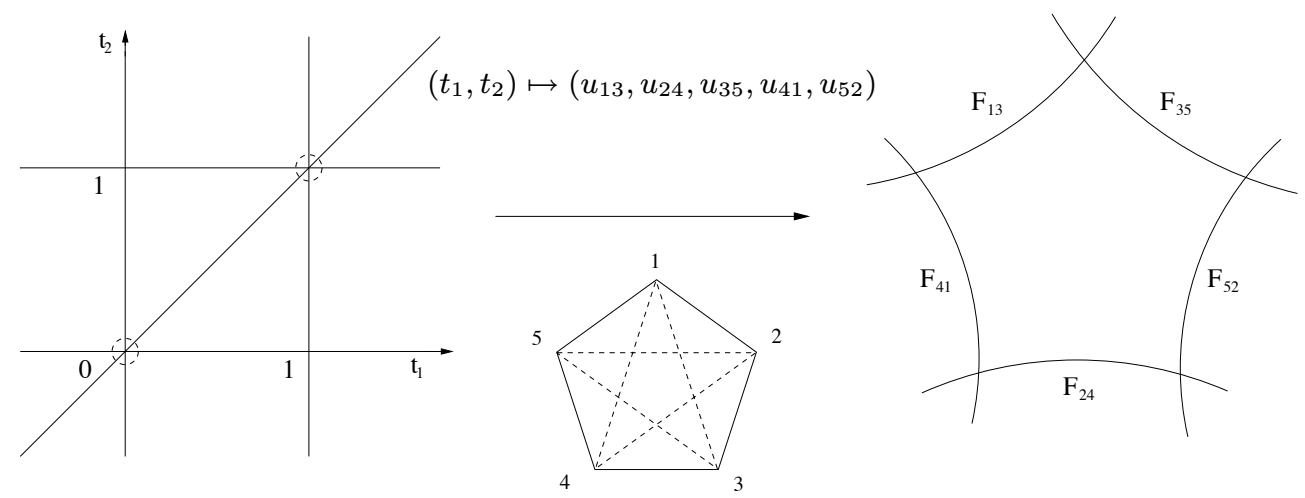

FIGURE 1. Dihedral coordinates on $\mathfrak{M}_{0,5}$. The scheme $\mathfrak{M}_{0,5}^{\delta}$ (right) is defined to be the Zariski closure of the image of the embedding $\left\{u_{i j}\right\}: \mathfrak{M}_{0,5} \hookrightarrow \mathbb{A}^{5}$ defined by the set of dihedral coordinates, which are indexed by chords in a pentagon (middle). This map has the effect of blowing up the points $(0,0)$ and $(1,1)$. A cell $X_{S, \delta}$ is given by the region $0<t_{1}<t_{2}<1$ (left). After blowing-up it becomes a pentagon with sides $F_{i j}=\left\{u_{i j}=0\right\}$.

Now consider the set of real points $\mathfrak{M}_{0, S}(\mathbb{R})$. There is a bounded cell $X_{S, \delta} \subset \mathfrak{M}_{0, S}(\mathbb{R})$ defined by the region $\left\{0<u_{i j}<1\right\}$. One shows that $\mathfrak{M}_{0, S}(\mathbb{R})$ is the disjoint union of the open cells $X_{S, \delta}$ of dimension $\ell=n-3$, as $\delta$ runs over the set of dihedral structures on $S$, so a dihedral structure corresponds to choosing a connected component of $\mathfrak{M}_{0, S}(\mathbb{R})$. The closure of the cell $\bar{X}_{S, \delta}$ satisfies

$$
\bar{X}_{S, \delta}=\left\{0 \leq u_{i j} \leq 1\right\} \subset \mathfrak{M}_{0, S}^{\delta}(\mathbb{R}),
$$

and $\mathfrak{M}_{0, S}^{\delta} \backslash \mathfrak{M}_{0, S}$ is the union of all divisors meeting the boundary of $X_{S, \delta}$. Therefore $\bar{X}_{S, \delta}$ is a convex polytope, and its boundary divisors give an explicit algebraic model of the associahedron. It is well-known that the combinatorics of the associahedron is given by triangulations of polygons. But because dihedral coordinates are already defined in terms of polygons, the main combinatorial properties of the associahedron, and its dihedral symmetry, follow 
immediately from properties of the coordinates $u_{i j}$. In particular, the face $F_{i j}=\left\{u_{i j}=0\right\}$ of $\bar{X}_{S, \delta}$ is a product

$$
F_{i j} \cong \bar{X}_{T_{1}, \delta_{1}} \times \bar{X}_{T_{2}, \delta_{2}}
$$

where $\left(T_{1}, \delta_{1}\right),\left(T_{2}, \delta_{2}\right)$ are two smaller polygons obtained by cutting the $n$-gon $S$ along the chord $\{i, j\}$ (fig. $3, \S 2.2$ ). In this way, a vertex $v$ of $\bar{X}_{S, \delta}$ corresponds to a complete triangulation $\alpha$ of the $n$-gon by $\ell$ chords. We also introduce explicit vertex coordinates $x_{1}^{\alpha}, \ldots, x_{\ell}^{\alpha}$ which are a certain subset of the set of all dihedral coordinates. These form a system of normal parameters in the neighbourhood of the vertex $v \in \bar{X}_{S, \delta}$ corresponding to $\alpha$, such that $\mathfrak{M}_{0, S}(\mathbb{R}) \subset \overline{\mathfrak{M}}_{0, S}(\mathbb{R})$ is locally the complement of the normal crossing divisor $x_{1}^{\alpha} \ldots x_{\ell}^{\alpha}=0$. These systems of coordinates (in the sense of differential geometry) are precisely what is needed for solving differential equations on $\mathfrak{M}_{0, S}$ and regularising logarithmic singularities of multiple polylogarithms.

In Section 3, we define an abstract algebra of iterated integrals on $\mathfrak{M}_{0, S}$ using a variant of Chen's reduced bar construction. Since this construction exists in far greater generality, we consider the complement of an arbitrary affine hyperplane arrangement defined over a field $k$ of characteristic 0 . So let

$$
M=\mathbb{A}^{\ell} \backslash \bigcup_{i=1}^{N} H_{i},
$$

where $H_{1}, \ldots, H_{N}$ is any set of hyperplanes in $\mathbb{A}^{\ell}$. Let $t_{1}, \ldots, t_{\ell}$ denote coordinates on $\mathbb{A}^{\ell}$, and let $\mathcal{O}_{M}$ denote the ring of regular functions on $M$. It is a differential $k$-algebra with respect to the coordinate derivations $\partial / \partial t_{i}$, for $1 \leq i \leq \ell$. Let

$$
\omega_{i}=\frac{d \alpha_{i}}{\alpha_{i}}, \quad \text { for } 1 \leq i \leq N,
$$

denote the logarithmic 1-form corresponding to $H_{i}$, where $\alpha_{i}$ is a defining equation for $H_{i}$. The version of the bar construction $B(M)$ we consider is defined as follows. Let $V_{m}(M)$ denote the $k$-vector space generated by linear combinations of symbols

$$
\sum_{I=\left(i_{1}, \ldots, i_{m}\right)} c_{I}\left[\omega_{i_{1}}|\ldots| \omega_{i_{m}}\right], \quad c_{I} \in k
$$

which satisfy the integrability condition:

$$
\sum_{I} c_{I} \omega_{i_{1}} \otimes \cdots \otimes\left(\omega_{i_{j}} \wedge \omega_{i_{j+1}}\right) \otimes \cdots \otimes \omega_{i_{m}}=0 \quad \text { for all } 1 \leq j<m .
$$

We then set $B(M)=\mathcal{O}_{M} \otimes_{k} \bigoplus_{m \geq 0} V_{m}(M)$, where $V_{0}(M)=k$. This is a graded Hopf algebra over $\mathcal{O}_{M}$ which is related to the zero ${ }^{\text {th }}$ cohomology group of the bar complex $\mathcal{O}(M) \otimes_{k} H^{0}\left(B\left(\Omega^{\bullet} \mathcal{O}_{M}\right)\right)$ studied by Chen [10] (see also [31]). We systematically drop the $H^{0}$ from the notation and simply write $B(M)$. Using the 1-part of the coproduct on $B(M)$, we define the action of $\ell$ commuting derivations $\partial_{i}$ on $B(M)$, and show that $\left(B(M), \partial_{i}\right)$ defines a differential extension of $\left(\mathcal{O}_{M}, \partial / \partial t_{i}\right)$. Its cohomology will be denoted $H_{\mathrm{DR}}^{\bullet}(B(M))$. The possibility of using iterated integrals to construct a Picard-Vessiot theory on manifolds was first suggested by Chen [9]. 
Theorem 1.2. $-B(M)$ is an infinite unipotent Picard-Vessiot extension of $\mathcal{O}_{M}$. In other words, it has no non-trivial differential ideals and its ring of constants is $k$. It is therefore a polynomial algebra. Furthermore, B( $M)$ contains 1-primitives:

$$
H_{\mathrm{DR}}^{1}(B(M))=0 .
$$

It follows that every unipotent extension of $B(M)$ is trivial, and it is the smallest extension of $\mathcal{O}_{M}$ with this property. Equivalently, $B(M)$ is the limit

$$
B(M)=\lim _{\rightarrow} U
$$

where $U$ ranges over all unipotent extensions $U$ of $\mathcal{O}_{M}$. In this sense it is universal, and it follows that its differential Galois group is a pro-unipotent group. Now if we identify $\mathfrak{M}_{0, S}$ with the affine hyperplane configuration

$$
\mathfrak{M}_{0, S} \cong\left\{\left(t_{1}, \ldots, t_{\ell}\right) \in \mathbb{A}^{\ell}: \quad t_{i} \neq 0,1, \quad t_{i}-t_{j} \neq 0\right\},
$$

then we define the universal algebra of polylogarithms on $\mathfrak{M}_{0, S}$ to be $B\left(\mathfrak{M}_{0, S}\right)$. In general, it is difficult to construct words (1.7) satisfying the integrability condition (1.8) since they rapidly become very complicated as the weight increases. In order to overcome this problem, we consider two affine hyperplane arrangements, one of which fibres linearly over the other. Therefore, let $M \subset \mathbb{A}^{\ell}$ and $M^{\prime} \subset \mathbb{A}^{\ell-1}$ denote two affine arrangements, and consider a linear projection

$$
\pi: M \rightarrow M^{\prime}
$$

with constant fibres $F$, where $F$ is the affine line $\mathbb{A}^{1}$ minus a number of marked points. We then prove that there is a tensor product decomposition

$$
B(M) \cong B\left(M^{\prime}\right) \otimes_{\mathcal{O}_{M^{\prime}}} B_{M^{\prime}}(F),
$$

where $B_{M^{\prime}}(F)$ is a free shuffle algebra which can be described explicitly. In the case of moduli spaces $\mathfrak{M}_{0, S}$, we apply this argument to the fibration map:

$$
\mathfrak{M}_{0, n} \longrightarrow \mathfrak{M}_{0, n-1}
$$

and use induction to deduce that $B\left(\mathfrak{M}_{0, S}\right)$ is a tensor product of free shuffle algebras. As a result, one can write down a basis for $B\left(\mathfrak{M}_{0, S}\right)$, and one deduces that the higher cohomology groups of $B\left(\mathfrak{M}_{0, S}\right)$ vanish.

Theorem 1.3. - The de Rham cohomology of $B\left(\mathfrak{M}_{0, S}\right)$ is trivial:

$$
H_{\mathrm{DR}}^{i}\left(B\left(\mathfrak{M}_{0, S}\right)\right)=0 \quad \text { for all } \quad i \geq 1 .
$$

A similar result holds for any hyperplane arrangement of fibre type, i.e., one which can be obtained as a sequence of such fibrations. In an appendix we also prove that $H_{\mathrm{DR}}^{i}(B(M))$, for $i \geq 1$, vanishes for all arrangements $M$ which have quadratic cohomology. The proofs only use simple arguments of differential algebra. Theorem 1.3 holds because $\mathfrak{M}_{0, S}$ is a $K(\pi, 1)$-rational space. An equivalent theorem is due to Hain and MacPherson ([33], [38]).

Given any point $z_{0} \in \mathfrak{M}_{0, S}(\mathbb{C})$ we define a realisation

$$
\begin{aligned}
\rho_{z_{0}}: B\left(\mathfrak{M}_{0, S}\right) & \stackrel{\sim}{\longrightarrow} L_{z_{0}}\left(\mathfrak{M}_{0, S}\right) \\
\sum_{I} f_{I}\left[\omega_{i_{1}}|\ldots| \omega_{i_{m}}\right] & \mapsto \sum_{I} f_{I} \int_{z_{0}}^{z} \omega_{i_{m}} \ldots \omega_{i_{1}},
\end{aligned}
$$

$4^{\text {e }}$ SÉRIE - TOME $42-2009-\mathrm{N}^{\mathrm{o}} 3$ 
given by iterated integration along any path $\gamma:[0,1] \rightarrow \mathfrak{M}_{0, S}(\mathbb{C})$ which begins at $z_{0}$ and ends at a variable point $z \in \mathfrak{M}_{0, S}(\mathbb{C})$. The integrability condition (1.8) ensures that the iterated integral (1.9) only depends on the homotopy class of $\gamma$. It therefore defines a multivalued function of the parameter $z$, i.e., a holomorphic function on the universal covering space of $\mathfrak{M}_{0, S}(\mathbb{C})$. Here, $L_{z_{0}}\left(\mathfrak{M}_{0, S}\right)$ is a differential graded algebra of multi-valued functions on $\mathfrak{M}_{0, S}$. We deduce from the previous theorem that $\ell$-forms with coefficients in $L_{z_{0}}\left(\mathfrak{M}_{0, S}\right)$ have primitives in $L_{z_{0}}\left(\mathfrak{M}_{0, S}\right)$.

The realisation $\rho_{z_{0}}$ is not quite good enough, however. We actually need a realisation $\rho_{z_{0}}: B\left(\mathfrak{M}_{0, S}\right) \rightarrow L_{z_{0}}\left(\mathfrak{M}_{0, S}\right)$, where the base point $z_{0}$ does not lie in $\mathfrak{M}_{0, S}(\mathbb{C})$. The point $z_{0}$ can be replaced with a tangential base point in the sense of [13], but our approach consists instead of viewing $z_{0}$ as the corner of a manifold with corners. This gives rise to divergent integrals, and to deal with this requires a regularisation procedure. The best approach is to consider the generating series of all such iterated integrals, and regularise them all simultaneously. Such a generating series satisfies a formal differential equation, and to solve it requires a generalised Fuchs' theorem in several variables in the unipotent case. For want of a suitable reference, we develop the necessary theory from scratch in Section 4. We also study the regularisation of logarithmic singularities along the boundary of any manifold with corners. Section 5 is devoted to a detailed study of the case of a one dimensional arrangement $\mathbb{P}^{1} \backslash\left\{\sigma_{0}, \ldots, \sigma_{N}, \infty\right\}$. In this case, the bar construction can be written down explicitly (it is a free shuffle algebra), and the corresponding iterated integrals are known as hyperlogarithms, which go back to Poincaré and Lappo-Danilevsky.

In Section 6, we apply all the results developed previously to the case of the moduli spaces $\mathfrak{M}_{0, S}$ to obtain the necessary regularisation results. The generating series of multiple polylogarithms can be described as follows. To each dihedral coordinate, or chord, is associated a logarithmic one-form

$$
\omega_{i j}=d \log u_{i j}, \quad \text { for } \quad\{i, j\} \in \chi_{S, \delta}
$$

It is symmetric in $i$ and $j$. Let $\delta_{i j}$, for $\{i, j\} \in \chi_{S, \delta}$, denote a set of symbols satisfying $\delta_{i j}=\delta_{j i}$, and consider the formal 1-form

$$
\Omega_{S, \delta}=\sum_{\{i, j\} \in \chi_{S, \delta}} \delta_{i j} \omega_{i j}
$$

This is a homogeneous version of the Knizhnik-Zamolodchikov form [36, 37, 18]. The integrability of $\Omega_{S, \delta}$ is equivalent to certain quadratic relations in the $\delta_{i j}$, which we call the dihedral braid relations. In the special case $\mathfrak{M}_{0,5}$, these reduce to the relations:

$$
\left[\delta_{i j}, \delta_{k l}\right]=0
$$

for any pair of chords $\{i, j\},\{k, l\}$ which do not cross, and the pentagonal relation

$$
\left[\delta_{13}, \delta_{24}\right]+\left[\delta_{24}, \delta_{35}\right]+\left[\delta_{35}, \delta_{41}\right]+\left[\delta_{41}, \delta_{52}\right]+\left[\delta_{52}, \delta_{13}\right]=0
$$


Let us fix a dihedral structure $\delta$ on $S$, and let $\widehat{\mathfrak{B}}_{S, \delta}(\mathbb{C})$ denote the ring of non-commutative formal power series in the symbols $\delta_{i j}$ with coefficients in $\mathbb{C}$, modulo the dihedral braid relations. Then we can consider the formal differential equation

$$
d L=\Omega_{S, \delta} L,
$$

where $L$ takes values in $\widehat{\mathfrak{B}}_{S, \delta}(\mathbb{C})$.

Theorem 1.4. - Let $v$ denote a vertex of the associahedron $\bar{X}_{S, \delta}$, and let $F_{v}$ denote the set of faces meeting $v$. Then there is a unique solution $L_{v, \delta}$ of (1.11) such that

$$
L_{v, \delta}(z)=f_{v, \delta}(z) \exp \left(\sum_{\{i, j\}: F_{i j} \in F_{v}} \delta_{i j} \log u_{i j}\right),
$$

where $f_{v, \delta}(z)$ is holomorphic in a neighbourhood of $v \in \mathfrak{M}_{0, S}^{\delta}$, and $f_{v, \delta}(v)=1$.

In other words, the function $L_{v, \delta}(z)$ is holomorphic on an open set of $\mathfrak{M}_{0, S}^{\delta}(\mathbb{C})$ which contains the real cell $X_{S, \delta}$, and has explicitly given monodromy around each face $u_{i j}=0$ of the associahedron $\bar{X}_{S, \delta}$ which meets the vertex $v$. The differential equation (1.11) is closely related to the Knizhnik-Zamolodchikov equation. Solutions to the latter equation are usually constructed by induction using fibration maps between configuration spaces. The previous theorem, however, is proved directly using the generalised Fuchs' theorem developed in Section 4. This approach has many advantages: firstly, there are no coherence conditions to verify; secondly, we obtain a direct geometric interpretation of Drinfeld's asymptotic zones, which were studied by Kapranov; and thirdly, the functoriality of the solution $L_{v, \delta}(z)$ with respect to maps between moduli spaces follows automatically. As a result, we obtain a direct definition of an associator on $\mathfrak{M}_{0, S}$ by considering the quotient of two different solutions:

$$
Z^{v, v^{\prime}}=\left(L_{v, \delta}(z)\right)^{-1} L_{v^{\prime}, \delta}(z) \in \widehat{\mathfrak{B}}_{S, \delta}(\mathbb{C}) .
$$

Here, $z$ is any point in an open neighbourhood of $X_{S, \delta}$ in $\mathfrak{M}_{0, S}^{\delta}$. The quotient is necessarily constant. The main properties of Drinfeld's associator can be derived immediately. Using the previous theorem, we deduce an expression for the monodromy of $L_{v, \delta}(z)$ and its regularisation in terms of the series $Z^{v, v^{\prime}}(\S 6.5)$. Then, using explicit expressions for hyperlogarithms, we deduce the following result, which was first proved by Le and Murakami, following Kontsevich.

THEOREM 1.5. - The coefficients of the series $Z^{v, v^{\prime}}$ are multiple zeta values.

It follows that the holonomy of the moduli spaces $\mathfrak{M}_{0, S}$ can be expressed using multiple zeta values and the constant $2 \pi i$. Now define $L^{v, \delta}\left(\mathfrak{M}_{0, S}\right)$ to be the differential algebra generated by the coefficients of the series $L_{v, \delta}(z)$. We can then define the sought-after realisation $\rho_{v, \delta}$ which is regularised at the vertex $v$ of $\bar{X}_{S, \delta}$ :

$$
\rho_{v, \delta}: B\left(\mathfrak{M}_{0, S}\right) \stackrel{\sim}{\longrightarrow} L^{v, \delta}\left(\mathfrak{M}_{0, S}\right),
$$

and which is defined over the field $k=\mathbb{Q}$. From this we deduce the main regularisation theorem, which describes the regularised restriction of a multiple polylogarithm to the face of the associahedron in terms of multiple zeta values. 
Theorem 1.6. - Let $F_{i j}$ denote a face of $\bar{X}_{S, \delta}$ isomorphic to a product $\bar{X}_{T_{1}, \delta_{1}} \times \bar{X}_{T_{2}, \delta_{2}}$ as in (1.6) above. Then if the vertex $v$ corresponds to the pair $\left(v_{1}, v_{2}\right)$,

$$
\operatorname{Reg}\left(L^{v, \delta}\left(\mathfrak{M}_{0, S}\right), F_{i j}\right) \otimes_{\mathbb{Q}} \mathcal{Z} \cong L^{v_{1}, \delta_{1}}\left(\mathfrak{M}_{0, T_{1}}\right) \otimes_{\mathbb{Q}} L^{v_{2}, \delta_{2}}\left(\mathfrak{M}_{0, T_{2}}\right) \otimes_{\mathbb{Q}} \mathcal{Z}
$$

In other words, the regularisation of multiple polylogarithms along divisors at infinity is completely determined by the combinatorics of the associahedron.

In Section 7, we study period integrals on $\mathfrak{M}_{0, S}(\mathbb{R})$ in terms of dihedral coordinates. We first show that, up to multiplication by a rational number, there is a unique algebraic $\ell$-form $\omega_{S, \delta}$, which has neither zeros nor poles on $\mathfrak{M}_{0, S}^{\delta}$. This form is invariant under the natural action of the dihedral group. We deduce that one can write an arbitrary integral (1.3) as a linear combination of integrals

$$
I_{S, \delta}\left(\alpha_{i j}\right)=\int_{\bar{X}_{S, \delta}} \prod_{\{i, j\} \in \chi_{S, \delta}} u_{i j}^{\alpha_{i j}} \omega_{S, \delta},
$$

for some fixed dihedral structure $\delta$, where the indices $\alpha_{i j} \in \mathbb{Z}$. Such an integral converges if and only if the coefficients $\alpha_{i j}$ are all non-negative. In explicit coordinates, (1.12) can be written as a generalized Selberg integral

$$
I_{S, \delta}\left(\alpha_{i j}\right)=\int_{[0,1]^{\ell}} \prod_{i=1}^{\ell} x_{i}^{a_{i}}\left(1-x_{i}\right)^{b_{i}} \prod_{i<j}\left(1-x_{i} x_{i+1} \ldots x_{j}\right)^{c_{i j}} d x_{1} \ldots d x_{\ell} .
$$

Particular subfamilies of these kinds of integrals have been considered by various authors in connection with the Diophantine approximation of zeta values (see, e.g., [54, 55, 21]). Terasoma has also computed the Taylor expansions (with respect to the exponents) of certain families of such integrals, and proved they are multiple zeta values [49]. The advantage of the blown-up integral representation (1.12) is that all poles of the integrand have been pushed to infinity, which allows an algebraic interpretation of the integrals as periods, and a systematic procedure for computing them, which is detailed in Section 8 and summarised below. As a further application of dihedral coordinates, we give an explicit formula for the order of vanishing of any form

$$
\prod_{\{i, j\} \in \chi_{S, \delta}} u_{i j}^{\alpha_{i j}} \omega_{S, \delta}
$$

along the divisors at infinity in $\overline{\mathfrak{M}}_{0, S}$. Using this formula we retrieve a result, due to Goncharov and Manin, which gives the singular locus of a certain family of forms which correspond directly to multiple zeta values. Our method exploits the action of the symmetric group on $\mathfrak{M}_{0, S}$, and completely avoids the delicate calculation of blow-ups and the cancellation of singularities studied in [27]. In $\S 7.5$, we show how functorial maps

$$
f: \mathfrak{M}_{0, S} \longrightarrow \mathfrak{M}_{0, T_{1}} \times \mathfrak{M}_{0, T_{2}}
$$

where $T_{1}$ and $T_{2}$ satisfy certain conditions (\$2.10), give rise to generalised product formulae between multiple zeta values. More precisely, given any such map $f$, there is a set of dihedral structures $G_{f}$ on $S$ such that the following formula holds:

$$
\int_{X_{T_{1}, \delta_{1}}} \omega_{1} \times \int_{X_{T_{2}, \delta_{2}}} \omega_{2}=\sum_{\gamma \in G_{f}} \int_{X_{S, \gamma}} f^{*}\left(\omega_{1} \otimes \omega_{2}\right) .
$$


This expresses a product of periods as a $\mathbb{Q}$-linear combination of other periods. We compute two explicit examples of such maps $f$; one where $G_{f}$ is as large as possible, and the other when $G_{f}$ reduces to a single element. In the first case, $G_{f}$ is the set of $(p, q)$ shuffles where $p=\operatorname{dim} \mathfrak{M}_{0, T_{1}}$ and $q=\operatorname{dim} \mathfrak{M}_{0, T_{2}}$, and (1.13) gives rise to the shuffle product for multiple zeta values. In the second case, we show that (1.13), on applying an identity due to Cartier, gives rise to the stuffle relations for multiple zeta values. Thus both shuffle and stuffle relations can be regarded as two extreme cases of generalised product relations of geometric origin on moduli spaces.

The above results are put together in Section 8, where we give a proof of Theorem 1.1 using Stokes' formula as described above. We summarise the main points of the argument here. The regularisation results of Section 6 provide the existence of a graded algebra of multivalued functions $L\left(\mathfrak{M}_{0, S}\right)$ with the following properties:

1. The graded part of weight 0 of $L\left(\mathfrak{M}_{0, S}\right)$ consists of all regular algebraic functions on $\mathfrak{M}_{0, S}$ with coefficients in $\mathbb{Q}$.

2. Primitives of $\ell$-forms exist in $L\left(\mathfrak{M}_{0, S}\right)$, and increase the weight by one.

3. The restriction of a function $f \in L\left(\mathfrak{M}_{0, S}\right)$ to a face of $\bar{X}_{S, \delta}$ is a product of multiple zeta values with functions in $L\left(\mathfrak{M}_{0, T_{1}}\right) L\left(\mathfrak{M}_{0, T_{2}}\right)$.

The argument for computing the period integrals is then by an inductive application of Stokes' theorem over the associahedron $\bar{X}_{S, \delta}$. At each stage, we must compute

$$
I=\int_{\bar{X}_{S, \delta}} f \omega_{S, \delta}
$$

where $f \in L\left(\mathfrak{M}_{0, S}\right)$ is a function which is allowed logarithmic singularities along the boundary $\partial \bar{X}_{S, \delta}$, but which has no polar singularities. Such an integral necessarily converges, and it follows from property (2) that there exists a primitive $F$ with coefficients in $L\left(\mathfrak{M}_{0, S}\right)$ such that $d F=f$. However, such primitives are not unique, and we may inadvertently have introduced extra poles. We show, however, that there exists a primitive $F$ with no poles along $\bar{X}_{S, \delta}$, and it then follows that $F$ extends continuously to the boundary $\partial \bar{X}_{S, \delta}$. The essential remark is that the one-form

$$
\log x d x \quad \text { where } x \geq 0,
$$

has a logarithmic singularity at the point $x=0$, but that its primitive $x \log x-x$ extends continuously to the point 0 . We can therefore restrict the primitive $F$ to the faces of the associahedron by property (3), and proceed by induction using Stokes' formula and (1.6) without any further difficulty. In $\S 8.5$ we show how the same strategy can be used to compute all relative periods (1.3) of moduli spaces $\mathfrak{M}_{0, S}$ when the integrand is an algebraic $\ell$-form, and finish with some simple examples in $\S 8.6$. The paper is completely self-contained, apart from some properties of iterated integrals which are very clearly presented in [31], and some remarks on framed motives in $\S 7.2$.

We expect that the ideas and methods introduced in this paper should have applications in the following situations. First of all, one can consider more general hyperplane configurations associated to other root systems or Coxeter groups, and consider the corresponding polylogarithm algebras, periods and associators. Notably, one can introduce $N^{\text {th }}$ roots of unity to obtain a tower of spaces over $\mathbb{P}^{1} \backslash\left\{0, e^{2 i \pi k / N}, \infty\right\}$ which are finite covers of $\mathfrak{M}_{0, S}$ and construct a similar theory giving a higher dimensional version of [14, 45]. Furthermore, 
in perturbative quantum field theory, it is generally believed that certain renormalised period integrals one derives from a large class of Feynman diagrams should give multiple zeta values. After blowing up, these are integrals of rational algebraic forms over algebraic convex polytopes. It would be very interesting to try to apply the methods of this paper to such integrals.

This paper was written during my doctoral thesis at the university of Bordeaux. I am very grateful to Richard Hain for his many detailed comments regarding an earlier version of this manuscript, Joseph Oesterlé for a thorough reading of $\S 2$, and especially to Pierre Cartier, without whose many suggestions, good humour, and continuous encouragement, this paper would not have reached its present form.

\section{Dihedral coordinates on $\mathfrak{M}_{0, n}$}

2.1. - Let $n \geq 4$, and let $S$ denote a set with $n$ elements. Let $\mathfrak{M}_{0, S}$ denote the moduli space of curves of genus 0 with $n$ points labelled with elements of $S$. If $\left(\mathbb{P}^{1}\right)_{*}^{S}$ denotes the set of all $n$-tuples of distinct points $z_{s} \in \mathbb{P}^{1}$, for $s \in S$, then

$$
\mathfrak{M}_{0, S}=\mathrm{PSL}_{2} \backslash\left(\mathbb{P}^{1}\right)_{*}^{S},
$$

where $\mathrm{PSL}_{2}$ is the algebraic group of automorphisms of $\mathbb{P}^{1}$ and acts by Möbius transformations. The quotient $\mathfrak{M}_{0, S}$ is an affine variety of dimension $\ell=n-3$. A point in $\mathfrak{M}_{0, S}(\mathbb{C})$ is therefore an injective map $S \hookrightarrow \mathbb{P}^{1}(\mathbb{C})$ considered up to the action of $\mathrm{PSL}_{2}(\mathbb{C})$. If $S=\left\{s_{1}, \ldots, s_{n}\right\}$, then we frequently write $i$ instead of $s_{i}$, and denote $\mathfrak{M}_{0, S}$ by $\mathfrak{M}_{0, n}$.

We wish to write down the set of regular functions on $\mathfrak{M}_{0, S}$, or, equivalently, the set of $\mathrm{PSL}_{2}$-invariant regular functions on $\left(\mathbb{P}^{1}\right)_{*}^{n}$. Let $i, j, k, l$ denote any distinct indices in $S$. Recall that the cross-ratio is defined by the formula:

$$
[i j \mid k l]=\frac{\left(z_{i}-z_{k}\right)\left(z_{j}-z_{l}\right)}{\left(z_{i}-z_{l}\right)\left(z_{j}-z_{k}\right)}
$$

The cross-ratios do not depend on the choice of coordinates $z_{i}$ and are $\mathrm{PSL}_{2}$-invariant. We therefore have a set of maps $[i j \mid k l]: \mathfrak{M}_{0, S} \rightarrow \mathfrak{M}_{0,4} \cong \mathbb{P}^{1} \backslash\{0,1, \infty\}$. The symmetric group on four letters $\mathfrak{S}_{4}$ acts on each cross-ratio via the group of anharmonic substitutions $\langle z \mapsto 1-z, z \mapsto 1 / z\rangle \cong \mathfrak{S}_{3} \cong \mathfrak{S}_{4} / V$, where $V$ is the Vierergruppe. We have:

$$
\begin{gathered}
{[i j \mid k l]=1-[i k \mid j l], \quad \text { and }[i j \mid l k]=[i j \mid k l]^{-1}=[j i \mid k l],} \\
\quad \text { and }[i j \mid k l]=[k l \mid i j]=[j i \mid l k]=[l k \mid j i] .
\end{gathered}
$$

For any five distinct indices $i, j, k, l, m \in S$ there is also the multiplicative relation:

$$
[i j \mid k l]=[i j \mid k m] \cdot[i j \mid m l] .
$$

In order to make explicit computations, it will be convenient to fix a system of coordinates on $\mathfrak{M}_{0, S}$ from the beginning. This breaks the symmetry, so we assume here that $S=\{1, \ldots, n\}$. Since the action of $\mathrm{PSL}_{2}$ is triply transitive on $\mathbb{P}^{1}$, we can place the coordinates $z_{1}$ at $1, z_{2}$ at $\infty$, and $z_{3}$ at 0 . We define simplicial coordinates $t_{1}, \ldots, t_{\ell}$ on $\mathfrak{M}_{0, S}$ by setting

$$
t_{1}=z_{4}, \ldots, t_{\ell}=z_{n}
$$


This identifies $\mathfrak{M}_{0, S}$ with the complement of the affine hyperplane configuration:

$$
\mathfrak{M}_{0, S} \cong\left\{\left(t_{1}, \ldots, t_{\ell}\right) \in \mathbb{A}^{\ell}: t_{i} \notin\{0,1\}, \quad t_{i} \neq t_{j} \text { for all } i \neq j\right\} .
$$

If we now perform the change of variables

$$
t_{1}=x_{1} \ldots x_{\ell}, t_{2}=x_{2} \ldots x_{\ell}, \ldots, t_{\ell}=x_{\ell},
$$

then we can identify $\mathfrak{M}_{0, S}$ with the open complement of hypersurfaces:

$$
\mathfrak{M}_{0, S} \cong\left\{\left(x_{1}, \ldots, x_{\ell}\right) \in \mathbb{A}^{\ell}: x_{i} \notin\{0,1\}, \quad x_{i} \ldots x_{j} \neq 1 \text { for all } i<j\right\} .
$$

The coordinates $x_{1}, \ldots, x_{\ell}$ will be referred to as cubical coordinates and are well-suited to the study of polylogarithms on $\mathfrak{M}_{0, S}(\S 6)$. Simplicial and cubical coordinates are two extremal cases of more general systems of coordinates which we define in an invariant manner in $\S 2.10$. We shall pass freely between the two systems, especially when making comparisons with formulae existing in the literature. The change of coordinates (2.4) has the effect of blowing up the origin; the boundary divisors at the origin in (2.5) cross normally, but do not in (2.3).

\subsection{Dihedral coordinates on $\mathfrak{M}_{0, S}$}

Let $S$ be a finite set with $n \geq 4$ elements.

Definition 2.1. - A cyclic structure $\gamma$ on $S$ is a cyclic ordering of the elements of $S$, or equivalently, an identification of the elements of $S$ with the edges of an oriented $n$-gon modulo rotations. A dihedral structure $\delta$ on $S$ is an identification with the edges of an unoriented $n$-gon modulo dihedral symmetries.

When we write $S=\left\{s_{1}, \ldots, s_{n}\right\}$, it will carry the obvious dihedral structure unless stated otherwise. In this case, the group of permutations $\mathfrak{S}_{S}$ can be identified with the symmetric group $\mathfrak{S}_{n}$. The set of cyclic (resp. dihedral) structures on $S$ is then indexed by the set of cosets $\mathfrak{S}_{n} / C_{n}$ (resp. $\mathfrak{S}_{n} / D_{2 n}$ ), where $C_{n}$ and $D_{2 n}$ denote the cyclic and dihedral groups of orders $n$ and $2 n$ respectively. We will often represent a dihedral structure as a regular $n$-gon $(S, \delta)$ with edges labelled $1,2, \ldots, n$ in order. A number in parentheses $(i)$, where $i \in \mathbb{Z} / n \mathbb{Z}$, will denote the pair of adjacent edges $\{i, i+1\}$. We will represent this on the $n$-gon $(S, \delta)$ by labelling the vertices with the elements $(1),(2), \ldots,(n)$; the convention is that the vertex labelled $(i)$ meets the edges labelled $i$ and $i+1$ modulo $n$ (Figures 2 and 3 ).

Given a dihedral structure $\delta$ on $S$, we define coordinates on $\mathfrak{M}_{0, S}$ using a certain subset of the set of all cross-ratios as follows. Let $\chi_{S, \delta}$ denote the set of all $n(n-3) / 2$ unordered pairs $\{i, j\}, 1 \leq i, j \leq n$ such that $i, j, i+1, j+1$ are distinct modulo $n$ (i.e., $i, j$ are not consecutive modulo $n$ ). Each element $\{i, j\} \in \chi_{S, \delta}$ will be depicted as a chord joining the vertices $i$ and $j$ in the regular $n$-gon (fig. 2). We set

$$
u_{i j}=[i i+1 \mid j+1 j] \quad \text { for each } \quad\{i, j\} \in \chi_{S, \delta} .
$$

A priori, $u_{i j}$ seems to depend on the ordered quadruple $(i, i+1, j, j+1)$, but one verifies from (2.1) that it is invariant on interchanging both $i \leftrightarrow i+1$ and $j \leftrightarrow j+1$, and is also invariant under $i \leftrightarrow j$. It therefore only depends on the chord $\{i, j\}$ and in particular is symmetric in $i$ and $j$. Consequently, we obtain a regular morphism

$$
\left(u_{i j}\right)_{\{i, j\} \in \chi_{S, \delta}}: \mathfrak{M}_{0, S} \longrightarrow \mathfrak{M}_{0,4}^{n(n-3) / 2} \subset \mathbb{A}^{n(n-3) / 2} .
$$

$4^{\mathrm{e}}$ SÉRIE - TOME $42-2009-\mathrm{N}^{\mathrm{O}} 3$ 
A simple calculation in simplicial coordinates gives the explicit formulae:
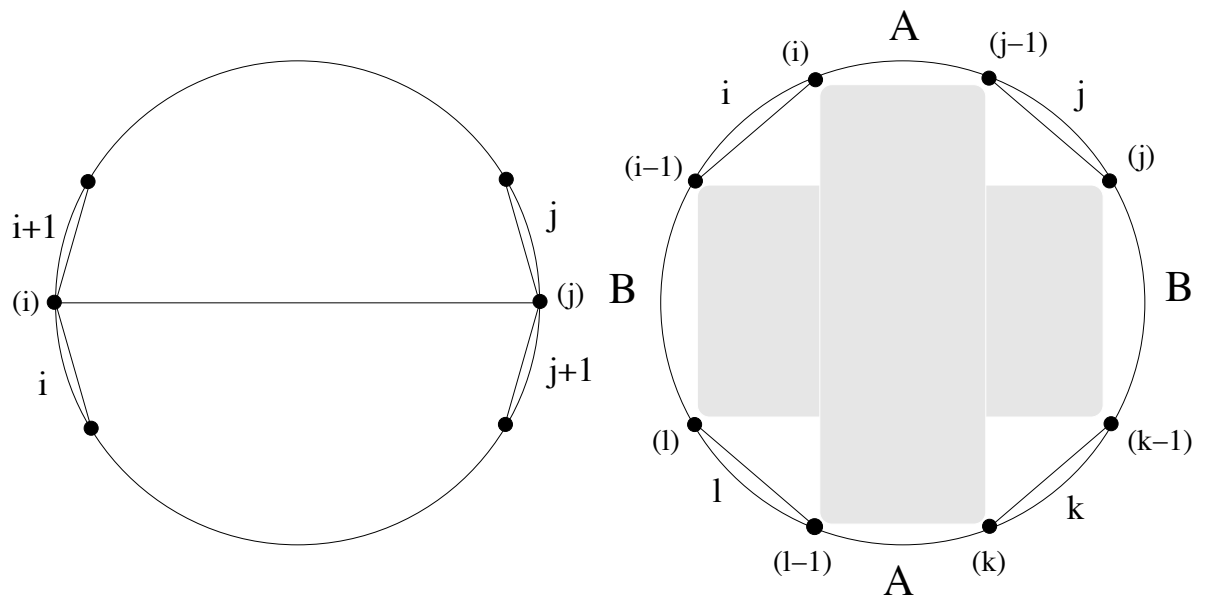

FIGURE 2. Part of an oriented regular $n$-gon inscribed in a circle. Its edges are labelled with the elements of $S$, and its vertices are labelled with elements of $S$ in parentheses. Left - a chord $\{i, j\} \in \chi_{S, \delta}$ meets four edges $i, i+1, j, j+1$ which define the dihedral coordinate $u_{i j}=[i i+1 \mid j+1 j]$. Changing the orientation of the $n$-gon does not alter $u_{i j}$ by the last equation in (2.1). Right - a set of four edges $i, j, k, l$ breaks the $n$-gon into four regions as in Lemma 2.2, and defines a pair $A, B \subset \chi_{S, \delta}$ of completely crossing chords, depicted by the shaded rectangles (Corollary 2.3).

$$
\begin{gathered}
u_{13}=1-t_{1}, \quad u_{2 n}=t_{\ell}, \quad u_{3 n}=\frac{t_{\ell}-t_{1}}{t_{\ell}\left(1-t_{1}\right)}, \\
u_{1 i}=\frac{1-t_{i-2}}{1-t_{i-3}}, 4 \leq i \leq n-1, \quad u_{2 i}=\frac{t_{i-3}}{t_{i-2}}, 4 \leq i \leq n-1, \\
u_{3 i}=\frac{t_{i-2}}{t_{i-3}} \frac{\left(t_{i-3}-t_{1}\right)}{\left(t_{i-2}-t_{1}\right)}, 5 \leq i \leq n-1, \quad u_{i n}=\frac{\left(1-t_{i-3}\right)\left(t_{i-2}-t_{\ell}\right)}{\left(t_{\ell}-t_{i-3}\right)\left(1-t_{i-2}\right)}, 4 \leq i \leq n-2 \\
u_{i j}=\frac{\left(t_{i-3}-t_{j-2}\right)\left(t_{i-2}-t_{j-3}\right)}{\left(t_{i-3}-t_{j-3}\right)\left(t_{i-2}-t_{j-2}\right)}, 4 \leq i<j \leq n-1 \text { and } j>i+1 .
\end{gathered}
$$

We will also require the following useful formulae:

$$
\begin{aligned}
t_{1} & =u_{24} \ldots u_{2 n}, \quad \ldots, \quad t_{\ell-1}=u_{2 n-1} u_{2 n}, \quad t_{\ell}=u_{2 n}, \\
1-t_{1} & =u_{13}, \quad 1-t_{2}=u_{13} u_{14}, \quad \ldots, \quad 1-t_{\ell}=u_{13} \ldots u_{1 n-1} .
\end{aligned}
$$

Similarly, the set of cubical coordinates $\left(x_{1}, \ldots, x_{\ell}\right)=\left(u_{24}, \ldots, u_{2 n}\right)$ is completely determined by the functions $u_{i j}$, and the following lemma shows that every cross-ratio can be written in terms of the functions $u_{i j}$, for $\{i, j\} \in \chi_{S, \delta}$.

Lemma 2.2. - Let $i, j, k, l$ be distinct indices modulo $n$ in dihedral order. Then

$$
[i j \mid k l]=\prod_{a=i}^{j-1} \prod_{b=k}^{l-1} u_{a b}^{-1} .
$$

Using (2.1), we can write any cross-ratio as a product of $u_{a b}$ or their inverses. 
Proof. - Suppose first that $1 \leq i<j<k<l \leq n$. Using the definition of $u_{a b}$, $u_{a k} \ldots u_{a l-1}=[a a+1 \mid k+1 k][a a+1 \mid k+2 k+1] \ldots[a a+1 \mid l l-1]=[a a+1 \mid l k]$, by repeated application of (2.2). Likewise, using (2.1) and (2.2),

$$
\prod_{a=i}^{j-1} \prod_{b=k}^{l-1} u_{a b}=[i i+1 \mid l k][i+1 i+2 \mid l k] \ldots[j-1 j \mid k+1 k]=[i j \mid l k] .
$$

The formula is clearly invariant under cyclic rotations. Therefore, given any four indices $i, j, k, l$ in arbitrary position, we can reduce to this case by applying the inversion (2.1), which allows us to interchange $i, j$, or $k, l$ or both pairs $(i, j),(k, l)$.

It follows from invariant theory that every $\mathrm{PSL}_{2}$-invariant regular function on $\left(\mathbb{P}^{1}\right)_{*}^{n}$ is a polynomial in the cross-ratios $[i j \mid k l]$. We deduce from Lemma 2.2 that the ring of regular functions on $\mathfrak{M}_{0, S}$ is generated by the dihedral coordinates $u_{i j}$, for $\{i, j\} \in \chi_{S, \delta}$, and their inverses.

We can write down a generating set for all algebraic relations between the coordinates $u_{i j}$ in a dihedrally-invariant manner. We say that two chords $\{i, j\}$ and $\{k, l\} \in \chi_{S, \delta}$ cross if they intersect in the interior of the polygon $(S, \delta)$. We write this

$$
\{i, j\} \sim_{\times}\{k, l\} .
$$

Given a subset $A \subset \chi_{S, \delta}$, let $A^{\times}$denote the set of chords in $\chi_{S, \delta}$ which cross every chord in $A$. We say that two sets $A, B \subset \chi_{S, \delta}$ cross completely if $A^{\times}=B$ and $B^{\times}=A$, i.e.,

$$
a \in A \Longleftrightarrow a \sim_{\times} b \quad \text { for all } b \in B
$$

and vice versa (fig. 2). If, for example, $A$ is the single chord $\{i, j\}$, and $B$ is the set of all chords crossing $\{i, j\}$, then $A$ and $B$ cross completely.

COROLlaRY 2.3. - For every two sets of chords $A, B \subset \chi_{S, \delta}$ which cross completely,

$$
u_{A}+u_{B}=1
$$

where $u_{A}=\prod_{a \in A} u_{a}$ and $u_{B}=\prod_{b \in B} u_{b}$.

Proof. - One can verify that $A$ and $B$ cross completely if and only if there exist four elements $\{i, j, k, l\} \subset S$ in dihedral order (fig. 2) such that

$$
\begin{aligned}
& A=\left\{\{p, q\} \in \chi_{S, \delta}: \quad i \leq p<j \quad \text { and } \quad k \leq q<l\right\} \\
& B=\left\{\{p, q\} \in \chi_{S, \delta}: \quad j \leq p<k \quad \text { and } \quad l \leq q<i\right\} .
\end{aligned}
$$

By Lemma 2.2 and (2.1), $u_{A}=[i j \mid k l]^{-1}=[i j \mid l k]$. Likewise, $u_{B}=[l i \mid j k]^{-1}=[i l \mid j k]$. It follows that $u_{A}+u_{B}=[i j \mid l k]+[i l \mid j k]=1$ by $(2.2)$. 


\subsection{The dihedral extension $\mathfrak{M}_{0, S}^{\delta}$}

Recall that $\mathfrak{M}_{0, S}$ is the affine scheme over $\mathbb{Z}$ in simplicial coordinates:

$$
\mathfrak{M}_{0, S}=\operatorname{Spec} \mathbb{Z}\left[t_{i}, \frac{1}{t_{i}}, \frac{1}{1-t_{i}}, \frac{1}{t_{i}-t_{j}}, 1 \leq i<j \leq \ell\right],
$$

where $\ell=|S|-3$, and we have the convention $\mathfrak{M}_{0, S}=\operatorname{Spec} \mathbb{Z}$ if $|S|=3$. Its dihedral extension $\mathfrak{M}_{0, S}^{\delta}$ is the affine scheme defined by the equations (2.10).

Definition 2.4. - Let $I_{S, \delta}^{\chi} \subset \mathbb{Z}\left[u_{i j}\right]$ denote the ideal generated by the identities (2.10). Let the dihedral extension $\mathfrak{M}_{0, S}^{\delta}$ of $\mathfrak{M}_{0, S}$ be the affine scheme

$$
\mathfrak{M}_{0, S}^{\delta}=\operatorname{Spec} \mathbb{Z}\left[u_{i j}:\{i, j\} \in \chi_{S, \delta}\right] / I_{S, \delta}^{\chi} .
$$

By Lemma 2.2, we could also define $\mathfrak{M}_{0, S}^{\delta}$ as follows:

$$
\mathfrak{M}_{0, S}^{\delta}=\operatorname{Spec} \mathbb{Z}[[i j \mid l k], \text { where } i, j, k, l \in S \text { are in dihedral order }] / I_{S, \delta},
$$

where $I_{S, \delta}$ is the ideal generated by the identities $[i j \mid l k]+[i l \mid j k]=1$ and (2.2).

For every chord $\{i, j\} \in \chi_{S, \delta}$ we define a divisor

$$
D_{i j}=\left\{u_{i j}=0\right\} \subset \mathfrak{M}_{0, S}^{\delta} .
$$

The scheme $\mathfrak{M}_{0, S}$ is retrieved from $\mathfrak{M}_{0, S}^{\delta}$ by removing all divisors $D_{i j},\{i, j\} \in \chi_{S, \delta}$.

Lemma 2.5. - There is a canonical open immersion $i_{\delta}: \mathfrak{M}_{0, S} \rightarrow \mathfrak{M}_{0, S}^{\delta}$ whose image is the complement $\mathfrak{M}_{0, S}^{\delta} \backslash \cup_{\{i, j\} \in \chi_{S, \delta}} D_{i j}$.

Proof. - Let $R_{u}=\mathbb{Z}\left[u_{i j},\{i, j\} \in \chi_{S, \delta}\right] / I_{S, \delta}^{\chi}$. Equations (2.8) define a map

$$
\phi: R_{u}\left[u_{i j}^{-1},\{i, j\} \in \chi_{S, \delta}\right] \longrightarrow \mathbb{Z}\left[t_{i}, \frac{1}{t_{i}}, \frac{1}{1-t_{i}}, \frac{1}{t_{i}-t_{j}}\right] .
$$

The way $\phi\left(u_{i j}\right)$ is defined is first to map $u_{i j}$ to the cross-ratio $[i i+1 \mid j+1 j]$, and then to set $z_{1}=1, z_{2}=\infty, z_{3}=0$ and $z_{i+3}=t_{i}$ for $1 \leq i \leq \ell$. The fact that $\phi$ is well-defined then follows from the proof of Lemma 2.2: each relation $\prod_{a \in A} u_{a}+\prod_{b \in B} u_{b}-1$ maps to an identity on cross-ratios of the form $[i j \mid l k]+[i l \mid j k]-1=0$.

In the other direction, we define a map

$$
\psi: \mathbb{Z}\left[t_{i}, \frac{1}{t_{i}}, \frac{1}{1-t_{i}}, \frac{1}{t_{i}-t_{j}}\right] \longrightarrow R_{u}\left[u_{i j}^{-1},\{i, j\} \in \chi_{S, \delta}\right]
$$

by $\psi\left(t_{i}\right)=u_{2 i+3} \ldots u_{2 n}$, for $1 \leq i \leq \ell$. It follows immediately from the second line of (2.8) that $\phi \circ \psi=1$. That $\psi \circ \phi=1$ can be verified case by case. Here we only do the generic case (the fourth line of (2.8)). Therefore, let $4 \leq i<j \leq n$, and $j>i+1$. Then $\psi \circ \phi\left(u_{i j}\right)$ is given by:

$$
\begin{aligned}
& \psi\left(\frac{\left(t_{i-3}-t_{j-2}\right)\left(t_{i-2}-t_{j-3}\right)}{\left(t_{i-3}-t_{j-3}\right)\left(t_{i-2}-t_{j-2}\right)}\right)=\frac{\left(u_{2 i} \ldots u_{2 n}-u_{2 j+1} \ldots u_{2 n}\right)\left(u_{2 i+1} \ldots u_{2 n}-u_{2 j} \ldots u_{2 n}\right)}{\left(u_{2 i} \ldots u_{2 n}-u_{2 j} \ldots u_{2 n}\right)\left(u_{2 i+1} \ldots u_{2 n}-u_{2 j+1} \ldots u_{2 n}\right)} \\
& =\frac{\left(u_{2 i} \ldots u_{2 j}-1\right)\left(u_{2 i+1} \ldots u_{2 j-1}-1\right)}{\left(u_{2 i} \ldots u_{2 j-1}-1\right)\left(u_{2 i+1} \ldots u_{2 j}-1\right)}=\frac{\prod_{I_{1}} u_{a b} \prod_{I_{2}} u_{a b}}{\prod_{I_{3}} u_{a b} \prod_{I_{4}} u_{a b}}=\prod_{3 \leq a \leq i-1} u_{a j}^{-1} \prod_{3 \leq a \leq i} u_{a j}=u_{i j}
\end{aligned}
$$


where $I_{1}=\{3 \leq a \leq i-1, j+1 \leq b \leq 1\}, I_{2}=\{3 \leq a \leq i, j \leq b \leq 1\}$, $I_{3}=\{3 \leq a \leq i-1, j \leq b \leq 1\}$, and $I_{4}=\{3 \leq a \leq i, j+1 \leq b \leq 1\}$, and where the indices are taken modulo $n$. The other cases are similar.

In order to describe the configuration of the divisors $D_{i j}$, consider cutting the regular $n$-gon along the chord $\{i, j\}$ joining vertices $(i)$ and $(j)$. This partitions the set of edges of $S$ into two sets $S_{1}$ and $S_{2}$ and breaks the $n$-gon into two smaller polygons. Their sets of edges are $S_{1} \cup\{e\}$ and $S_{2} \cup\{e\}$, where $e$ is the new edge given by the chord $\{i, j\}$ (fig. 3). Each set inherits a dihedral structure $\delta_{k}$ for $k=1,2$, and $\chi_{S, \delta}$ is a disjoint union:

$$
\chi_{S, \delta}=\chi_{S_{1} \cup\{e\}, \delta_{1}} \sqcup \chi_{S_{2} \cup\{e\}, \delta_{2}} \sqcup\{i, j\} \sqcup \bigcup_{\{k, l\} \sim_{\times}\{i, j\}}\{k, l\} .
$$
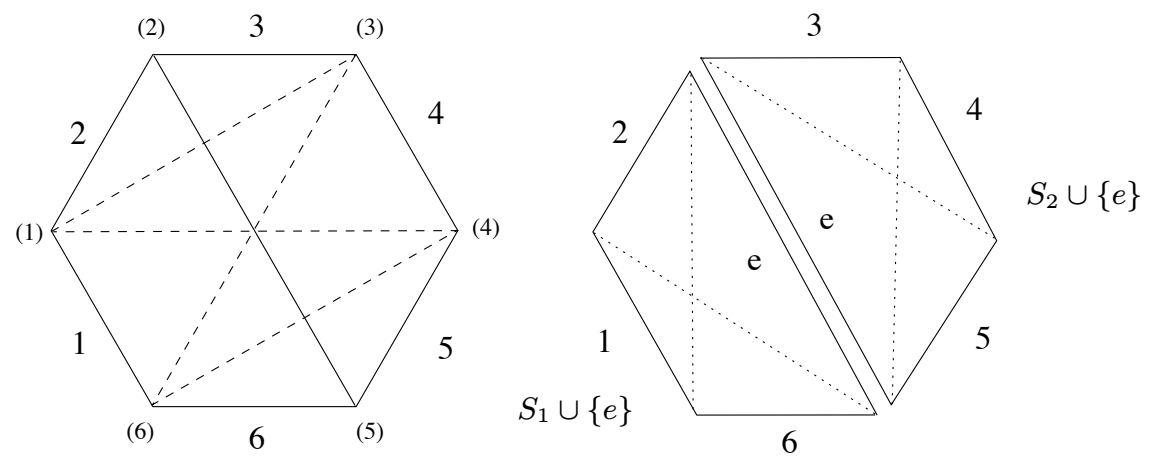

FIGURE 3. Decomposition of the hexagon on setting $u_{25}=0$. The variables corresponding to chords which cross $\{2,5\}$, namely $u_{13}, u_{46}, u_{14}, u_{36}$, are all equal to 1 (left). The system (2.10) splits into the pair of equations, $u_{15}=1-u_{26}$ and $u_{35}=1-u_{24}$, which identifies $D_{25}$ with $\mathfrak{M}_{0,4}^{\delta} \times \mathfrak{M}_{0,4}^{\delta}$.

Lemma 2.6. - The decomposition (2.14) gives a canonical isomorphism

$$
D_{i j} \cong \mathfrak{M}_{0, S_{1} \cup\{e\}}^{\delta_{1}} \times \mathfrak{M}_{0, S_{2} \cup\{e\}}^{\delta_{2}} .
$$

Proof. - Equations (2.10) imply in particular that

$$
u_{a b}+\prod_{\{c, d\} \sim_{x}\{a, b\}} u_{c d}=1 \quad \text { for all }\{a, b\} \in \chi_{S, \delta} .
$$

Therefore, setting $u_{i j}=0$ implies that $u_{k l}=1$ for all chords $\{k, l\}$ which cross $\{i, j\}$. The system of equations (2.10) then decomposes into two disjoint sets, each one containing all variables $u_{a b}$, where $\{a, b\} \in \chi_{S_{1} \cup\{e\}, \delta_{1}}$, or $\chi_{S_{2} \cup\{e\}, \delta_{2}}$ respectively. To see this, consider the equation

$$
u_{A}+u_{B}=1,
$$

where $A, B \subset \chi_{S, \delta}$ cross completely, and where we write $u_{I}=\prod_{i \in I} u_{i}$ for any subset $I \subset \chi_{S, \delta}$. Consider the decomposition (2.14) above, and set $A_{i}=A \cap \chi_{S_{i} \cup\{e\}, \delta_{i}}$ for $i=1,2$. 
It follows from the calculation above that, since $u_{i j}=0$,

$$
u_{A}= \begin{cases}0 & \text { if }\{i, j\} \in A \\ u_{A_{1}} u_{A_{2}} & \text { otherwise. }\end{cases}
$$

A similar formula holds for $u_{B}$. The picture below depicts the three possible cases which can occur, up to exchanging $i, j$ or $A, B$. If neither $A_{1}$ nor $A_{2}$ is empty, the set $A$ contains chords on either side of the chord $\{i, j\}$ (case I). It follows that $\{i, j\} \in A$, and therefore $u_{A}=0$. Since $B=A^{\times}$, it follows that $B \subset\{i, j\}^{\times}$, and so $u_{k l}=1$ for all $\{k, l\} \in B$. Thus (2.16) reduces to $0+1=1$. Therefore we can assume without loss of generality that $A_{1}=\varnothing$ (see cases II and III), and so $u_{A}=u_{A_{2}}$ by (2.17). It is clear that $B_{1}=\varnothing$, and so $u_{B}=u_{B_{2}}$.
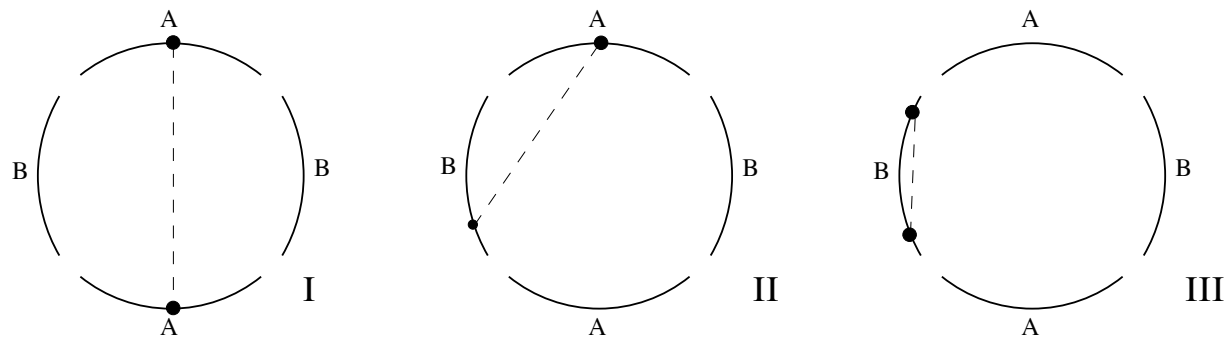

It follows that Equation (2.16) reduces to $u_{A_{2}}+u_{B_{2}}=1$, which is a defining equation for

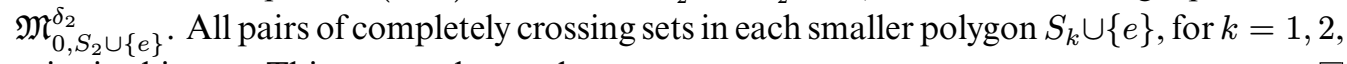
arise in this way. This proves the result.

It follows from the proof of the lemma that $D_{i j}$ and $D_{k l}$ have non-empty intersection if and only if the chords $\{i, j\}$ and $\{k, l\}$ do not cross. By (2.15), $u_{i j}$ and $u_{k l}$ cannot simultaneously be zero if $\{i, j\} \sim_{\mathrm{x}}\{k, l\}$. We are therefore led to consider partial decompositions of the $n-\operatorname{gon}(S, \delta)$ by $k$ non-crossing chords.

Definition 2.7. - For each integer $1 \leq k \leq \ell$, let $\chi_{S, \delta}^{k}$ denote the set of $k$ distinct chords $\alpha=\left\{\left\{i_{1}, j_{1}\right\}, \ldots,\left\{i_{k}, j_{k}\right\}\right\}$ in the $n$-gon $(S, \delta)$, such that no pair of chords in $\alpha$ cross. For each such $\alpha \in \chi_{S, \delta}^{k}$, let $D_{\alpha}$ denote the subvariety defined by the equations $u_{i_{1} j_{1}}=\cdots=$ $u_{i_{k} j_{k}}=0$, i.e., $D_{\alpha}=\bigcap_{m=1}^{k} D_{i_{m} j_{m}}$.

It follows by induction using the previous lemma that the codimension of $D_{\alpha}$, for $\alpha \in \chi_{S, \delta}^{k}$, is exactly $k$, and that every codimension- $k$ intersection of divisors $D_{i j}$ arises in this manner. Any set of $k$ chords $\alpha \in \chi_{S, \delta}^{k}$ splits the polygon into $k+1$ pieces, and we have:

$$
D_{\alpha} \cong \prod_{m=1}^{k+1} \mathfrak{M}_{0, S_{m}}^{\delta_{m}}
$$

where $\left(S_{m}, \delta_{m}\right)$ are given by the set of all edges of each small polygon in the $k$-decomposition $\alpha$, with the induced dihedral structures (fig. 4). 


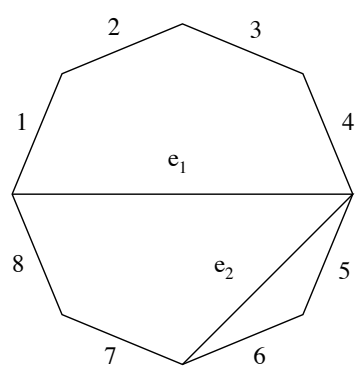

FIGURE 4. A partial decomposition $\alpha \in \chi_{8, \delta}^{2}$ of an octagon gives an isomorphism of $D_{\alpha}$ with $\mathfrak{M}_{0,5}^{\delta_{1}} \times \mathfrak{M}_{0,4}^{\delta_{2}} \times \mathfrak{M}_{0,3}^{\delta_{3}}=\mathfrak{M}_{0,5}^{\delta_{1}} \times \mathbb{A}^{1} \times\{\mathrm{pt}\}$.

REMARK 2.8. - The set of all polygons equipped with the operation of gluing sides together forms what is known as the mosaic operad [15]. This says that, given two polygons with edges labelled $S_{1} \cup\{e\}$ and $S_{2} \cup\{e\}$ respectively, there is an operation of gluing along the common edge $e$, which gives rise to a map

$$
\chi_{S_{1} \cup\{e\}, \delta_{1}}^{k} \times \chi_{S_{2} \cup\{e\}, \delta_{2}}^{l} \rightarrow \chi_{S_{1} \cup S_{2}, \delta}^{k+l+1} .
$$

This corresponds to the decomposition of Lemma 2.6.

\subsection{Forgetful maps between moduli spaces and projections}

Let $T$ denote any subset of $S$ such that $|T| \geq 3$. There is a natural map

$$
f_{T}: \mathfrak{M}_{0, S} \longrightarrow \mathfrak{M}_{0, T}
$$

obtained by forgetting the marked points of $S$ which do not lie in $T$. Now suppose that $S$ has dihedral structure $\delta$. Then $T$ inherits a dihedral structure which we denote $\delta_{T}$. Identifying $S$ with the set of edges of the $n$-gon $(S, \delta)$, we obtain a map:

$$
f_{T}: \chi_{S, \delta} \rightarrow \chi_{T, \delta_{T}}
$$

which contracts all edges in $S \backslash T$ and combines the corresponding chords (fig. 5).

Lemma 2.9. - The map (2.19) extends to give a map $f_{T}: \mathfrak{M}_{0, S}^{\delta} \longrightarrow \mathfrak{M}_{0, T}^{\delta_{T}}$ such that

$$
f_{T}^{*}\left(u_{k l}\right)=\prod_{\{a, b\} \in f_{T}^{-1}(\{k, l\})} u_{a b} .
$$

Proof. - By (2.12), $f_{T}^{*}$ is induced by the map:

$$
f_{T}^{*}: \mathbb{Z}\left[[i j \mid l k]: i, j, k, l \in T^{\delta_{T}}\right] / I_{T, \delta_{T}} \longrightarrow \mathbb{Z}\left[[i j \mid l k]: i, j, k, l \in S^{\delta}\right] / I_{S, \delta},
$$

where $i, j, k, l \in T^{\delta_{T}}$ (resp. $S^{\delta}$ ) denotes four elements in $T$ (resp. $S$ ) in dihedral order. Formula (2.20) follows immediately from Lemma 2.2.

Remark 2.10. - If $\{i, j\} \in \chi_{S, \delta}$, let $T$ denote the four element set $T=\{i, i+1, j, j+1\}$. Then the dihedral coordinate $u_{i j}$ is by definition a forgetful map:

$$
\mathfrak{M}_{0, S}^{\delta} \stackrel{f_{T}}{\longrightarrow} \mathfrak{M}_{0, T}^{\delta_{T}} \cong \mathbb{A}^{1} .
$$




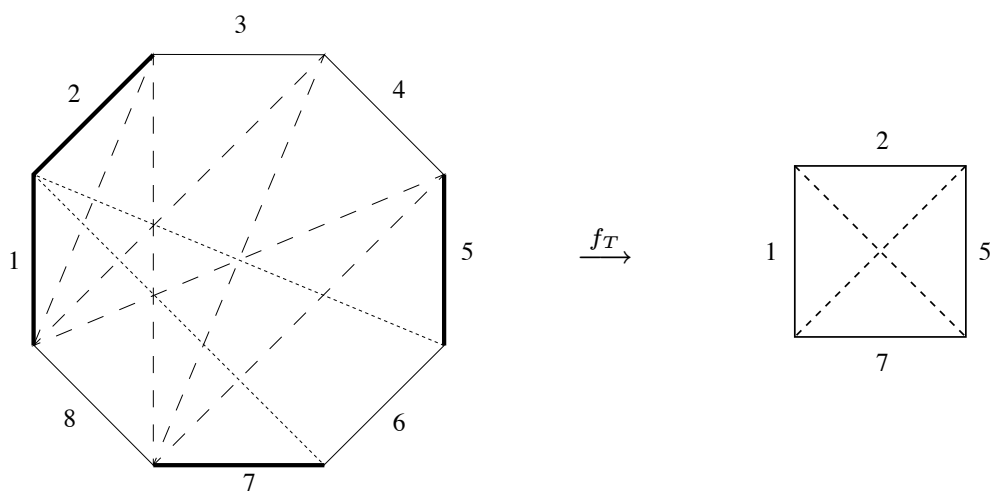

Figure 5. The forgetful map $f_{T}$ contracts edges labelled $3,4,6,8$. The dihedral coordinates corresponding to the two chords in the square are pulled back by $f_{T}^{*}$ to $u_{15} u_{16}$ and $u_{27} u_{37} u_{47} u_{28} u_{38} u_{48}$.

If $T_{1}, T_{2}$ are two subsets of $S$ such that $\left|T_{1} \cap T_{2}\right| \geq 3$, we obtain a map

$$
f_{T_{1}} \times f_{T_{2}}: \mathfrak{M}_{0, S}^{\delta} \longrightarrow \mathfrak{M}_{0, T_{1}}^{\delta_{1}} \times_{\mathfrak{M}_{0, T_{1} \cap T_{2}}^{\delta^{\prime}}} \mathfrak{M}_{0, T_{2}}^{\delta_{2}}
$$

where $\delta_{1}=\delta_{T_{1}}, \delta_{2}=\delta_{T_{2}}$, and $\delta^{\prime}=\delta_{T_{1} \cap T_{2}}$. Recall that $\mathfrak{M}_{0,3}^{\delta}=\operatorname{Spec} \mathbb{Z}$.

For future reference, we will need to consider what happens when $\left|T_{1} \cap T_{2}\right|=2$. Suppose that the elements of $T_{k}$ are consecutive with respect to $\delta$, for $k=1,2$. Then two cases can occur: either $T_{1} \cap T_{2}$ consists of two consecutive elements $\{i, i+1\}$ where $i \in S$, or $T_{1} \cap T_{2}$ has two components and $T_{1} \cap T_{2}=\{a, b\}$, where $a, b \in S$ are non-consecutive. We only consider the first case here. This corresponds to choosing a directed chord $\{i, j\} \in \chi_{S, \delta}$, and cutting along it. Let $S_{1}$ and $S_{2}$ denote the corresponding partition of the set $S$ viewed as edges of the $n$-gon (fig. 3), and consider the larger overlapping sets defined by $T_{1}=S_{1} \cup\{i, i+1\}$ and $T_{2}=S_{2} \cup\{i, i+1\}$. A product of forgetful maps gives

$$
f_{T_{1}} \times f_{T_{2}}: \mathfrak{M}_{0, S}^{\delta} \longrightarrow \mathfrak{M}_{0, T_{1}}^{\delta_{1}} \times \mathfrak{M}_{0, T_{2}}^{\delta_{2}} .
$$

The dimension of the product on the right hand side is $\left(\left|T_{1}\right|-3\right)+\left(\left|T_{2}\right|-3\right)$, which is $\ell-1$, one less than on the left. Suppose that the chord $\{i, j\}=\{i, i+2\}$ is short. In that case, one of the sets, say $T_{2}$, has just three elements, and $\mathfrak{M}_{0, T_{2}}^{\delta_{2}}$ reduces to a point. The complement $S \backslash T_{1}$ is a single point. We write $S=\left\{s_{1}, \ldots, s_{n}\right\}$, and let $\left\{s_{n}\right\}=S \backslash T_{1}$. In that case, the restriction of $f_{T_{1}}$ to $\mathfrak{M}_{0, S}$ :

$$
f_{T_{1}}: \mathfrak{M}_{0,\left\{s_{1}, \ldots, s_{n}\right\}} \rightarrow \mathfrak{M}_{0,\left\{s_{1}, \ldots, s_{n-1}\right\}}
$$

is a fibration with one-dimensional fibres which are isomorphic to the punctured projective line $\mathbb{P}^{1} \backslash\left\{s_{1}, \ldots, s_{n-1}\right\}$. In general, the restriction of the map (2.22) to the open set $\mathfrak{M}_{0, S}$ is not a fibration, but almost. Let us compute it in cubical coordinates. By applying a dihedral symmetry, we can assume $i=2$. By (2.9), we have $u_{2 j}=x_{m}$, where $m=j-3$. One verifies that

$$
\begin{aligned}
& \mathfrak{M}_{0, T_{1}} \cong\left\{\left(x_{1}, \ldots, x_{m-1}\right): x_{i} \notin\{0,1\}, \quad x_{i} \ldots x_{j} \neq 1 \quad \text { for } i<j\right\} \\
& \mathfrak{M}_{0, T_{2}} \cong\left\{\left(x_{m+1}, \ldots, x_{\ell}\right): x_{i} \notin\{0,1\}, \quad x_{i} \ldots x_{j} \neq 1 \quad \text { for } i<j\right\}
\end{aligned}
$$


and the map $f_{T_{1}} \times f_{T_{2}}: \mathfrak{M}_{0, S} \rightarrow \mathfrak{M}_{0, T_{1}} \times \mathfrak{M}_{0, T_{2}}$ is just projection onto $x_{m}=0$ :

$$
f_{T_{1}} \times f_{T_{2}}:\left(x_{1}, \ldots, x_{\ell}\right) \mapsto\left(\left(x_{1}, \ldots, x_{m-1}\right),\left(x_{m+1}, \ldots, x_{\ell}\right)\right) .
$$

We can therefore think of $(2.22)$ as a coordinate projection in cubical coordinates. Referring to Figure 10, we see that in $\mathfrak{M}_{0,6}$ there are two such types of map, one given by projection onto $\mathfrak{M}_{0,5}$ (set $x_{1}=0$ or $x_{3}=0$ ), and the other given by projection onto $\mathfrak{M}_{0,4} \times \mathfrak{M}_{0,4}$ (set $x_{2}=0$ ). Restricting (2.22) to the divisor $u_{i j}=0$, we retrieve the isomorphism $D_{i j} \cong$ $\mathfrak{M}_{0, T_{1}}^{\delta_{1}} \times \mathfrak{M}_{0, T_{2}}^{\delta_{2}}$ which was defined in Lemma 2.6.

REMARK 2.11. - One can make the map $f_{T_{1}} \times f_{T_{2}}: \mathfrak{M}_{0, S} \rightarrow \mathfrak{M}_{0, T_{1}} \times \mathfrak{M}_{0, T_{2}}$ into a fibration by restricting it to an open subset $U_{S} \subset \mathfrak{M}_{0, S}$. One obtains a map $f_{T_{1}} \times f_{T_{2}}$ : $U_{S} \rightarrow V_{S}$, where $V_{S} \subset \mathfrak{M}_{0, T_{1}} \times \mathfrak{M}_{0, T_{2}}$, whose fibres are isomorphic to $\mathbb{A}^{1}$ with $N$ points removed. The $N$ removed points correspond to the set of chords $\{k, l\}$ which cross $\{i, j\}$, plus the chord $\{i, j\}$ itself.

For example, consider the case $\mathfrak{M}_{0,6}$, where we write the cubical coordinates $\left(x_{1}, x_{2}, x_{3}\right)$ as $(x, y, z)$. Then $U_{S}=\mathfrak{M}_{0,6} \backslash\{x=z\}$, and $V_{S}=\left\{(x, z) \in \mathbb{A}^{2}: x, z \neq 0,1: x \neq z^{ \pm 1}\right\}$. Then the fibration map $(x, y, z) \mapsto(x, z): U_{S} \rightarrow V_{S}$ has fibres $\left\{y \in \mathbb{A}^{1}: y \notin\left\{0,1, x^{-1}, z^{-1},(x z)^{-1}\right\}\right\}$. The removed points in the fibre are given by the five equations $u_{25}=0, u_{13}=u_{14}=u_{36}=$ $u_{46}=1$ (see Figure 3).

\subsection{Smoothness and irreducibility of $\mathfrak{M}_{0, S}^{\delta}$.}

Let $S=\{1, \ldots, n\}$, with canonical dihedral structure $\delta$, and let $S_{1}=S \backslash\{n\}$, $S_{2}=\{1,2, n-1, n\}$, with induced dihedral structures $\delta_{1}, \delta_{2}$. This gives a product of forgetful maps

$$
f_{S_{1}} \times f_{S_{2}}: \mathfrak{M}_{0, S}^{\delta} \longrightarrow \mathfrak{M}_{0, S_{1}}^{\delta_{1}} \times \mathfrak{M}_{0, S_{2}}^{\delta_{2}} \cong \mathfrak{M}_{0, S_{1}}^{\delta_{1}} \times \mathbb{A}^{1}
$$

We will show that this map induces an isomorphism between an open subset of $\mathfrak{M}_{0, S}^{\delta}$ and an open subset of $\mathfrak{M}_{0, S_{1}}^{\delta_{1}} \times \mathbb{A}^{1}$, and deduce that $\mathfrak{M}_{0, S}^{\delta}$ is smooth and irreducible by induction. Note that $f_{S_{1}}: \mathfrak{M}_{0, S} \rightarrow \mathfrak{M}_{0, S_{1}}$ is just the map which forgets the $n^{\text {th }}$ marked point, and its fibre is isomorphic to the projective line minus $n-1$ points.

Let us define

$$
R_{u}=\mathbb{Z}\left[u_{i j}:\{i, j\} \in \chi_{S, \delta}\right] / I_{S, \delta}^{\chi} \quad \text { and } \quad R_{v}=\mathbb{Z}\left[v_{i j}:\{i, j\} \in \chi_{S_{1}, \delta_{1}}\right] / I_{S_{1}, \delta_{1}}^{\chi},
$$

so that $u_{i j}$, for $1 \leq i, j \leq n$ are dihedral coordinates on $\mathfrak{M}_{0, S}^{\delta}=\operatorname{Spec} R_{u}$ and $v_{i j}$, for $1 \leq i, j \leq n-1$ are dihedral coordinates on $\mathfrak{M}_{0, S_{1}}^{\delta_{1}}=\operatorname{Spec} R_{v}$. Let

$$
v=\left(1-v_{13} v_{14} \ldots v_{1 n-2} t\right) \ldots\left(1-v_{1 n-3} v_{1 n-2} t\right)\left(1-v_{1 n-2} t\right) \in R_{v}[t],
$$

and write $u=u_{2 n} u_{3 n} \ldots u_{n-3 n} \in R_{u}$. Note that $u=1-u_{1 n-1} u_{1 n-2}$ in $R_{u}$.

Proposition 2.12. - The map $f_{S_{1}} \times f_{S_{2}}$ induces an isomorphism

$$
\mathfrak{M}_{0, S}^{\delta} \backslash\{u=0\} \cong\left(\mathfrak{M}_{0, S_{1}}^{\delta_{1}} \times \mathbb{A}^{1}\right) \backslash\{v=0\} .
$$


Proof. - Using (2.20), the morphism $\left(f_{S_{1}} \times f_{S_{2}}\right)^{*}$ is given by:

$$
\begin{aligned}
\phi: R_{v}[t]\left[v^{-1}\right] & \longrightarrow R_{u}\left[u^{-1}\right], \\
\phi(t) & =u_{1 n-1}, \\
\phi\left(v_{i n-1}\right) & =u_{i n-1} u_{i n}, \quad \text { for } 2 \leq i \leq n-3, \\
\phi\left(v_{i j}\right) & =u_{i j}, \quad \text { if } i, j \neq n-1 .
\end{aligned}
$$

One checks from the definitions that

$$
\phi(v)=\left(1-u_{13} u_{14} \ldots u_{1 n-1}\right) \ldots\left(1-u_{1 n-2} u_{1 n-1}\right)=u_{2 n}^{n-4} u_{3 n}^{n-5} \ldots u_{n-3 n} .
$$

We define an inverse map:

$$
\begin{aligned}
\psi: R_{u}\left[u^{-1}\right] & \longrightarrow R_{v}[t]\left[v^{-1}\right] . \\
\psi\left(u_{1 n-1}\right) & =t \\
\psi\left(u_{2 n} u_{3 n} \ldots u_{i n}\right) & =1-v_{1 i+1} \ldots v_{1 n-2} t, \quad \text { for } 2 \leq i \leq n-2, \\
\psi\left(u_{i n-1} u_{i n}\right) & =v_{i n-1}, \\
\psi\left(u_{i j}\right) & =v_{i j}, \quad \text { if } i, j \notin\{n-1, n\} .
\end{aligned}
$$

These equations determine $\psi$ uniquely, using the fact that $\psi$ is multiplicative, and the fact that $u$ and $v$ are invertible. To prove that $\psi$ is well-defined, one must show that $\psi$ maps every relation $\prod_{a \in A} u_{a}+\prod_{b \in B} u_{b}-1$ to 0 , where $A, B$ are sets of completely crossing chords in $(S, \delta)$. One checks that the image under $\psi$ of such a relation is of degree at most one in the variable $t$, so it suffices to check the cases $t=0$, and $t=1$. Setting $t=0$ in the definition of $\psi$ gives rise to a map:

$$
\psi_{0}: R_{u}\left[u^{-1}\right] \longrightarrow R_{v}\left[v^{-1}\right],
$$

where $\psi_{0}\left(u_{1 n-1}\right)=0, \psi_{0}\left(u_{i j}\right)=1$ if $i=n$ or $j=n$, and $\psi_{0}\left(u_{i j}\right)=v_{i j}$ for all other $i, j$. This map is well-defined since it is nothing other than the restriction to an open subset of the inclusion map:

$$
\mathfrak{M}_{0, S_{1}}^{\delta_{1}} \cong D_{1 n-1}=\left\{u_{1 n-1}=0\right\} \rightarrow \mathfrak{M}_{0, S}^{\delta} \text {. }
$$

Similarly, setting $t=1$ in the definition of $\psi$ gives rise to another map

$$
\psi_{1}: R_{u}\left[u^{-1}\right] \longrightarrow R_{v}\left[v^{-1}\right]
$$

where $\psi_{1}\left(u_{n n-2}\right)=0, \psi\left(u_{i j}\right)=1$ if $i=n-1$ or $j=n-1$, and $\psi\left(u_{i n}\right)=v_{i n-1}$ and $\psi\left(u_{i j}\right)=v_{i j}$ for all other $i, j$. This is just the inclusion $D_{n n-2} \rightarrow \mathfrak{M}_{0, S}^{\delta}$, which proves that $\psi_{1}$ is also well-defined.

It is clear from the definitions that $\psi \circ \phi$ is the identity on $\left(R_{v} \otimes_{\mathbb{Z}} \mathbb{Z}[t]\right)\left[v^{-1}\right]$. To show that $\phi \circ \psi$ is the identity on $R_{u}\left[u^{-1}\right]$, it suffices to verify that

$$
\phi \circ \psi\left(u_{2 n} u_{3 n} \ldots u_{i n}\right)=\phi\left(1-v_{1 i+1} \ldots v_{1 n-2} t\right)=1-u_{1 i+1} \ldots u_{1 n-1}=u_{2 n} \ldots u_{i n} .
$$

The last equality holds since $\{\{1, i+1\}, \ldots,\{1, n-1\}\}$ and $\{\{2, n\}, \ldots,\{i, n\}\}$ are completely crossing chords. Thus $\phi$ and $\psi$ are isomorphisms, inverse to one another, which completes the proof.

For every vertex $(i)$ in $(S, \delta)$, let $Z_{i}=\cup_{j} D_{i j}$, where the union is over all chords $\{i, j\} \in \chi_{S, \delta}$ meeting $(i)$. Let $U_{i}=\mathfrak{M}_{0, S}^{\delta} \backslash Z_{i}$ denote the open complement. 
Lemma 2.13. - The sets $U_{i}$, for $i \in S$, form an open cover of $\mathfrak{M}_{0, S}^{\delta}$.

Proof. - It suffices to show that $\bigcap_{i \in S} Z_{i}=\varnothing$. Let $S=\{1, \ldots, n\}$. We have

$$
\bigcap_{i \in S} Z_{i}=\bigcap_{i \in S} \bigcup_{j:\{i, j\} \in \chi_{S, \delta}} D_{i j}=\bigcup_{j_{1}, \ldots, j_{n}}\left(D_{1 j_{1}} \cap \cdots \cap D_{n j_{n}}\right)
$$

where the union is over $j_{1}, \ldots, j_{n}$ such that $\left\{1, j_{1}\right\}, \ldots,\left\{n, j_{n}\right\}$ are chords of $S$. At least two of these chords must necessarily cross (otherwise, there would exist a triangulation of an $n$-gon with a chord passing through each vertex, which is clearly impossible). Since the intersection of divisors $D_{i j}, D_{k l}$ corresponding to pairs of chords which cross is empty, it follows that $D_{1 j_{1}} \cap \cdots \cap D_{n j_{n}}=\varnothing$, for all such $j_{1}, \ldots, j_{n}$, and hence $\bigcap_{i \in S} Z_{i}=\varnothing$.

COROLLARY 2.14. - The scheme $\mathfrak{M}_{0, S}^{\delta}$ is integral and smooth.

Proof. - If $|S|=4$, then $\mathfrak{M}_{0, S}^{\delta} \cong \mathbb{A}^{1}$, which is clearly integral and smooth. By induction, assume that $\mathfrak{M}_{0, S}^{\delta}$ is integral and smooth for all $|S|=n-1$. If $|S|=n$, then it follows from Proposition 2.12 that the open set $\mathfrak{M}_{0, S}^{\delta} \backslash\{u=0\}$ is integral and smooth. The divisor $\{u=0\}$ is by definition $D_{2 n} \cup \cdots \cup D_{n-3 n}$ which is contained in $Z_{n}=D_{2 n} \cup \cdots \cup D_{n-2 n}$, and therefore $U_{n} \subset \mathfrak{M}_{0, S}^{\delta} \backslash\{u=0\}$. It follows that $U_{n}$ is integral and smooth, and likewise for every $U_{i}$ for $i \in S$, by dihedral symmetry. By the previous lemma, these sets form an open cover of $\mathfrak{M}_{0, S}^{\delta}$, which proves the corollary.

THEOREM 2.15. - The affine scheme $\mathfrak{M}_{0, S}^{\delta}$ is integral and smooth, and the divisors $D_{i j}$, for $\{i, j\} \in \chi_{S, \delta}$, are smooth and normal crossing.

Proof. - We know from Lemma 2.6 that each divisor $D_{i j}$ is isomorphic to a product of spaces $\mathfrak{M}_{0, S^{\prime}}^{\delta^{\prime}}$ and is therefore smooth. The fact that they cross normally follows by induction from the isomorphism (2.23) of proposition 2.12 .

Let $\alpha=\left\{\left\{i_{1}, j_{1}\right\}, \ldots,\left\{i_{k}, j_{k}\right\}\right\} \in \chi_{S, \delta}^{k}$ denote a decomposition of $(S, \delta)$, where $1 \leq k \leq \ell$. It suffices to check that the divisors $D_{i j}$, where $\{i, j\} \in \alpha$, cross normally in an open neighbourhood of $D_{\alpha}$ which is contained in $U_{m}$, for each $1 \leq m \leq n$. Without loss of generality we can assume that $m=n$. There are three cases. First of all, if $(n) \in(S, \delta)$ is a vertex which is an endpoint of a chord in $\alpha$, then $D_{\alpha} \cap U_{n}=\varnothing$, and there is nothing to check. Therefore we can assume that the vertex $(n)$ meets no chord in $\alpha$. Suppose that $\alpha$ contains the short chord $\{1, n-1\}$, and suppose that $\left\{i_{k}, j_{k}\right\}=\{1, n-1\}$. Under the

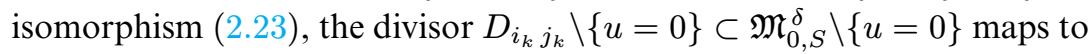

$$
\mathfrak{M}_{0, S_{1}}^{\delta_{1}} \times\{t=0\} \subset\left(\mathfrak{M}_{0, S_{1}}^{\delta_{1}} \times \mathbb{A}^{1}\right) \backslash\{v=0\},
$$

and the remaining divisors $D_{i_{r} j_{r}} \cap U$, for $1 \leq r \leq k-1$, map to a set of $k-1$ divisors $\left(D_{i_{r}^{\prime} j_{r}^{\prime}} \times \mathbb{A}^{1}\right) \backslash\{v=0\} \subset\left(\mathfrak{M}_{0, S_{1}}^{\delta_{1}} \times \mathbb{A}^{1}\right) \backslash\{v=0\}$ which cross normally, by induction hypothesis. Finally, if $(n)$ meets no chord in $\alpha$, and the short chord $\{1, n-1\}$ is not in $\alpha$, the same argument applies, except that all divisors in $\alpha$ map to divisors of the form $\left(D_{i_{r}^{\prime} j_{r}^{\prime}} \times \mathbb{A}^{1}\right) \backslash\{v=0\}$, and the conclusion is the same. Since the open sets $U_{1}, \ldots, U_{n}$ cover $\mathfrak{M}_{0, S}^{\delta}$, this proves that the divisors $D_{i j}$ are normal crossing. 


\subsection{Normal vertex coordinates on $\mathfrak{M}_{0, S}(\mathbb{C})$}

The previous theorem gives normal coordinates in the neighbourhood of any intersection of divisors $D_{\alpha}$ on $\mathfrak{M}_{0, S}^{\delta}$. Unfortunately, these coordinates are not canonical. For example, if $S=\{1, \ldots, 6\}$ and $\alpha=\{\{1,3\},\{1,5\},\{3,5\}\}$, then Proposition 2.12 yields, for example, a set of normal coordinates of the form $\left(u_{15}, u_{13} u_{36}, u_{35}\right)$, which are not symmetric. It is therefore reasonable to ask whether the dihedral coordinates $\left(u_{15}, u_{13}, u_{35}\right)$ themselves form a coordinate neighbourhood of $D_{\alpha}=\left\{u_{15}=u_{13}=u_{35}=0\right\}$. It turns out that they actually define a 2:1 étale map to $\mathbb{A}^{3}$ on a certain open subset of $\mathfrak{M}_{0, S}^{\delta}$. In this section, we study canonical normal coordinates in the neighbourhood of each subvariety $D_{\alpha}$, for general $\alpha \in \chi_{S, \delta}^{k}$.

We first consider two useful relations satisfied by the dihedral coordinates $u_{i j}$ on $\mathfrak{M}_{0, S}$. We frequently use the following notation: for any two sets $I, J \subset S$, we write

$$
u_{I J}=\prod_{i \in I, j \in J} u_{i j}
$$

Also, given two consecutive indices $i, i+1$ modulo $n$, we adopt the convention that $u_{i+1}=$ 0 . This is compatible with the decomposition of Lemma 2.6: after cutting along a chord $\{i, j\} \in \chi_{S, \delta}$, the vertices $i$ and $j$ become adjacent in each small polygon, and indeed, $u_{i j}=0$ is the equation of the corresponding divisor (Fig. 4).

Lemma 2.16. - Let $\{p, q\} \in \chi_{S, \delta}$. Then any three of the four coordinates $u_{p q}, u_{p q+1}$, $u_{p+1 q}, u_{p+1 q+1}$ determine the fourth, and we have the butterfly relation on $\mathfrak{M}_{0, S}$ :

$$
\frac{u_{p q}\left(1-u_{p q+1}\right)}{1-u_{p q} u_{p q+1}}=\frac{1-u_{p+1 q+1}}{1-u_{p+1 q+1} u_{p+1 q}}
$$

where $u_{p+1 q}=0\left(u_{p q+1}=0\right)$ if $p+1$ and $q$, (respectively $\left.q+1, p\right)$ are consecutive.

Proof. - Let $A$ and $B$ be the subsets of vertices $S$ pictured in the diagram below (left). Then (2.10) implies the following equations:

$$
\begin{aligned}
1-u_{p q+1} & =u_{A p+1} u_{A B} u_{A q} \\
1-u_{p q} u_{p q+1} & =u_{A p+1} u_{A B} \\
1-u_{p+1 q+1} & =u_{p B} u_{A B} u_{A q} u_{p q} \\
1-u_{p+1 q+1} u_{p+1 q} & =u_{p B} u_{A B} .
\end{aligned}
$$

Identity (2.27) follows by substitution.

Lemma 2.17. - Let $p, q, r$ denote three non-consecutive elements of $S$, and set $\pi_{r}=\prod_{p<i<q} u_{i r}$. Then the triangle relation holds on $\mathfrak{M}_{0, S}$ :

$$
\frac{1-u_{p q}}{\left(1-u_{p r}\right)\left(1-u_{q r}\right)}=\frac{\pi_{r}}{\left(1-\pi_{r} u_{p r}\right)\left(1-\pi_{r} u_{q r}\right)} .
$$

If we regard this as a quadratic equation for $\pi_{r}$ in $\mathbb{Q}\left(u_{p q}, u_{p r}, u_{q r}\right)$, then the discriminant is non-zero in a Zariski-open neighbourhood of $u_{p r}=u_{q r}=u_{p q}=0$. 

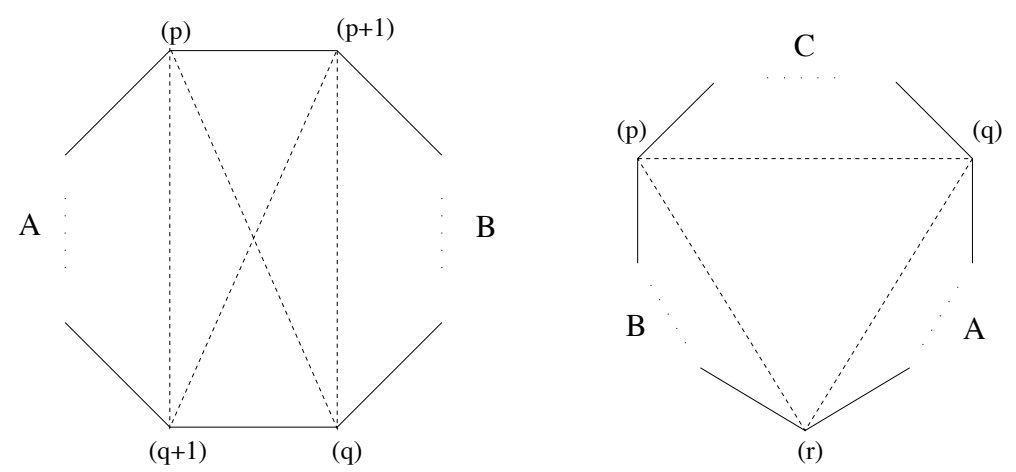

FIGURE 6. Proof of the butterfly (left) and triangle relation (right).

Proof. - Let $A, B$ and $C$ be the subsets of vertices $S$ pictured in the diagram above (right). Then (2.10) implies the following equations:

$$
\begin{aligned}
1-u_{p q} & =u_{B C} \pi_{r} u_{A C}, \\
1-u_{p r} & =u_{B C} u_{B q} u_{A B}, \\
1-u_{q r} & =u_{A C} u_{A p} u_{A B}, \\
1-u_{p r} \pi_{r} & =u_{B q} u_{A B}, \\
1-u_{q r} \pi_{r} & =u_{A p} u_{A B} .
\end{aligned}
$$

The identity (2.28) follows by substitution. One verifies by straightforward computation that the discriminant of $(2.28)$ is

$$
\Delta_{p q, r}=\left(1-u_{p q} u_{q r}+u_{q r} u_{p r}-u_{p r} u_{p q}\right)^{2}-4\left(1-u_{p q}\right)^{2} u_{p r} u_{q r},
$$

from which the last statement follows.

Let $\alpha \in \chi_{S, \delta}^{\ell}$ denote a triangulation of the $n$-gon $(S, \delta)$. An internal triangle of $\alpha$ is a triple $p, q, r \in S$ such that $p, q, r$ are non-adjacent, and $\{p, q\},\{p, r\}$, and $\{q, r\}$ are in $\alpha$. A free vertex of $\alpha$ is a vertex $i \in S$ such that $\{i, k\} \notin \alpha$ for all $\{i, k\} \in \chi_{S, \delta}$. If $t$ denotes the number of internal triangles in $\alpha$, and $v$ denotes the number of free vertices in $\alpha$, then it is easy to show that $v=2+t$. A triangulation of the $n$-gon has no internal triangles if and only if it has exactly two free vertices.

Definition 2.18. - Let $\alpha \in \chi_{S, \delta}^{\ell}$, and choose an ordering on the set of chords $\left\{i_{1}, j_{1}\right\}, \ldots,\left\{i_{\ell}, j_{\ell}\right\}$ in $\alpha$. Then the set of vertex coordinates ${ }^{(1)}$ corresponding to the ordered triangulation $\alpha$ is the set of variables:

$$
x_{1}^{\alpha}, \ldots, x_{\ell}^{\alpha},
$$

defined by setting $x_{k}^{\alpha}=u_{i_{k} j_{k}}$ for $1 \leq k \leq \ell$.

(1) The reason for this terminology will become apparent in $\S 2.7$. A triangulation $\alpha$ corresponds to the point $D_{\alpha}$ which is a vertex (corner) of the Stasheff polytope $\bar{X}_{S, \delta} \subset \mathfrak{M}_{0, S}^{\delta}(\mathbb{R})$ (see Fig. 10). 
If $\alpha=\{\{2,4\}, \ldots,\{2, n\}\}$ with the natural ordering, then $x_{k}^{\alpha}=x_{k}=u_{2 k+3}$ for $1 \leq k \leq \ell$, and we retrieve the cubical coordinates defined in (2.5) as a special case.

Let $\{i, j\} \in \chi_{S, \delta}$. Recall from (2.14) that there is a decomposition

$$
\chi_{S, \delta}=\chi^{\prime} \sqcup\{i, j\} \sqcup \bigcup_{\{k, l\} \sim_{\times}\{i, j\}}\{k, l\},
$$

where $\chi^{\prime}$ consists of all chords $\{a, b\}$ which do not cross $\{i, j\}$. The following lemma states that we can eliminate all dihedral coordinates $u_{k l}$, where $\{k, l\}$ crosses $\{i, j\}$.

Lemma 2.19. - Let $\{i, j\} \in \alpha$. On the open set $\mathfrak{M}_{0, S}^{\delta} \backslash\left\{u_{i j}=1\right\}$, every variable $u_{k l}$, where $\{k, l\} \in \chi_{S, \delta}$ crosses $\{i, j\}$, can be expressed as a rational function of $u_{i j}$, and the variables $u_{a b}$ where $\{a, b\} \in \chi^{\prime}$.

Proof. - The easiest way to see this is on the example $\alpha \in \chi_{8, \delta}^{5}$ depicted in Figure 7 (left), where $\{i, j\}=\{1,5\}$. Consider the following equations given by (2.10):

$$
\begin{aligned}
u_{28} & =1-u_{17} u_{16} u_{15} u_{14} u_{13}, \\
u_{28} u_{27} & =1-u_{16} u_{15} u_{14} u_{13}, \\
u_{28} u_{27} u_{26} & =1-u_{15} u_{14} u_{13} \\
u_{38} u_{28} & =1-u_{17} u_{16} u_{15} u_{14} \\
u_{38} u_{37} u_{28} u_{27} & =1-u_{16} u_{15} u_{14} \\
u_{38} u_{37} u_{36} u_{28} u_{27} u_{26} & =1-u_{15} u_{14}
\end{aligned}
$$

The identities (2.10) imply that

$$
1-u_{i j}=\prod_{\{k, l\} \sim \times\{i, j\}} u_{k l},
$$

and therefore all the variables on the left hand side in the equations above are invertible on $\mathfrak{M}_{0, S}^{\delta} \backslash\left\{u_{i j}=1\right\}$. All the variables on the right hand side lie in $\chi^{\prime} \cup\{i, j\}$. We can therefore solve for $u_{28}, u_{27}, u_{26}, u_{38}, u_{37}, u_{36}$ and so on, in turn. The general case is similar.

Let $\{i, j\} \in \chi_{S, \delta}$ denote a chord. Then $\{i, j\}$ partitions the set of edges of $(S, \delta)$ into two sets, $S_{1}$ and $S_{2}$. The chord itself corresponds to the four edges $E=\{i, i+1, j, j+1\}$. The sets $T_{1}=S_{1} \cup E$, and $T_{2}=S_{2} \cup E$ overlap in precisely the set $E$, and therefore $\left|T_{1} \cap T_{2}\right|=4$. Let $\delta_{E}$ denote the induced dihedral structure on $E$. By definition of the dihedral coordinate $u_{i j}$, there is an isomorphism $u_{i j}: \mathfrak{M}_{0, E}^{\delta_{E}} \cong \mathbb{A}^{1}$. Therefore (2.21) defines a map:

$$
\mathfrak{M}_{0, S}^{\delta} \longrightarrow \mathfrak{M}_{0, T_{1}}^{\delta_{1}} \times \mathbb{A}^{1} \mathfrak{M}_{0, T_{2}}^{\delta_{2}} .
$$

The chord $\{i, j\}$ is in both $\chi_{T_{1}, \delta_{1}}$ and $\chi_{T_{2}, \delta_{2}}$ (Fig. 7).

Proposition 2.20. - The map (2.30) defines an isomorphism

$$
\mathfrak{M}_{0, S}^{\delta} \backslash\left\{u_{i j}=1\right\} \cong \mathfrak{M}_{0, T_{1}}^{\delta_{1}} \backslash\left\{u_{i j}=1\right\} \times_{\mathbb{A}^{1} \backslash\{1\}} \mathfrak{M}_{0, T_{2}}^{\delta_{2}} \backslash\left\{u_{i j}=1\right\} .
$$


Proof. - The map $\left(f_{T_{1}} \times f_{T_{2}}\right)^{*}$ is given by:

$$
\begin{aligned}
\mathbb{Z}[[i j \mid l k]: i, j, k, l & \left.\in T_{1}^{\delta_{1}}\right] / I_{T_{1}, \delta_{1}} \otimes_{\mathbb{Z}\left[u_{i j}\right]} \mathbb{Z}\left[[i j \mid l k]: i, j, k, l \in T_{2}^{\delta_{2}}\right] / I_{T_{1}, \delta_{1}} \\
& \longrightarrow \mathbb{Z}\left[[i j \mid l k]: i, j, k, l \in S^{\delta}\right] / I_{S, \delta},
\end{aligned}
$$

with the notation used in the proof of Lemma 2.9. It can also be regarded as a map:

$$
\begin{gathered}
\mathbb{Z}\left[u_{a b}:\{a, b\} \in \chi_{T_{1}, \delta_{1}}\right] / I_{T_{1}, \delta_{1}}^{\chi} \otimes_{\mathbb{Z}\left[u_{i j}\right]} \mathbb{Z}\left[u_{a b}:\{a, b\} \in \chi_{T_{2}, \delta_{2}}\right] / I_{T_{2}, \delta_{2}}^{\chi} \\
\longrightarrow \mathbb{Z}\left[u_{a b}:\{a, b\} \in \chi_{S, \delta}\right] / I_{S, \delta}^{\chi},
\end{gathered}
$$

The previous lemma implies that the map $\left(f_{T_{1}} \times f_{T_{2}}\right)^{*}$ is surjective when we invert the coordinates $u_{k l}$ such that $\{k, l\}$ crosses $\{i, j\}$, i.e., on the open set $u_{i j} \neq 1$. Using this, one can write down an inverse map to $\left(f_{T_{1}} \times f_{T_{2}}\right)^{*}$, and check that it is indeed an inverse. We omit the details.
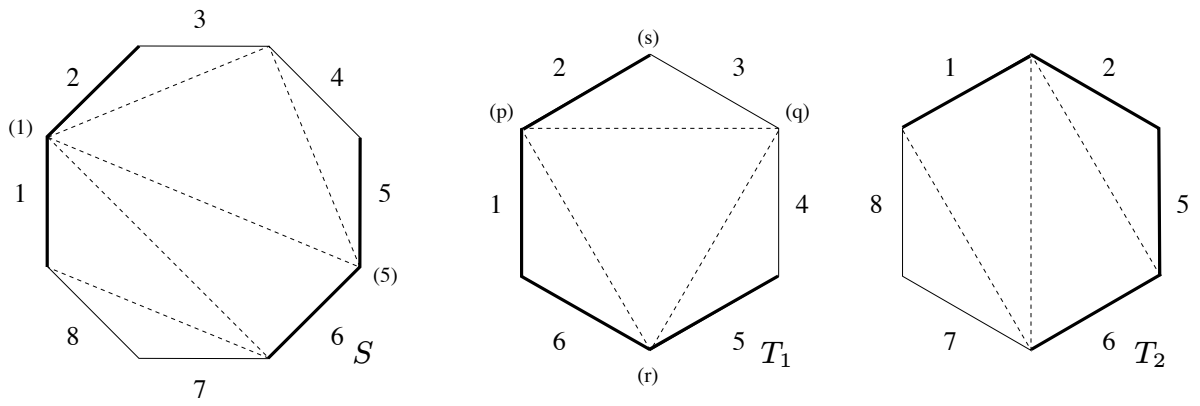

Figure 7. The induction step in the proof of Proposition 2.22. The chord $\{1,5\}$ in the octagon on the left distinguishes the four thick edges $E=\{1,2,5,6\}$. The two sets $T_{1}=\{1,2,3,4,5,6\}$ and $T_{2}=\{5,6,7,8,1,2\}$ intersect in $E$ and define the hexagons on the right. The right hand hexagon can be further decomposed into a pair of pentagons, but the middle hexagon has an internal triangle, so we have to invoke the triangle lemma 2.17. In this way, we reduce to the case of $\mathfrak{M}_{0,6}^{\delta}$ and $\mathfrak{M}_{0,5}^{\delta}$ only.

Each set of vertex coordinates defines an étale map on a certain Zariski-open subset obtained by iterating the map in the previous proposition. Let $\alpha \in \chi_{S, \delta}^{k}$ denote any partial $k$-decomposition of the $n$-gon and define:

$$
U_{\alpha}=\bigcap_{\{i, j\} \in \alpha}\left\{u_{i j} \neq 1\right\} \subset \mathfrak{M}_{0, S}^{\delta} .
$$

Since $D_{\alpha}=\left\{u_{i j}=0:\right.$ for $\left.\{i, j\} \in \alpha\right\}$, it follows that $D_{\alpha} \subset U_{\alpha}$, and $U_{\alpha}$ is an open neighbourhood of the subvariety $D_{\alpha}$ which contains the open set $\mathfrak{M}_{0, S}$.

Now let $\{i, j\} \in \alpha$, and consider the map (2.31). By restricting, we obtain two decompositions $\alpha_{1}, \alpha_{2}$, and Zariski-open sets $U_{\alpha_{1}}, U_{\alpha_{2}}$ on $T_{1}, T_{2}$ respectively.

REMARK 2.21. - The embedding (2.31) extends the isomorphism $D_{\alpha} \cong D_{\alpha_{1}} \times D_{\alpha_{2}}$. The product structure on each boundary stratum of $\mathfrak{M}_{0, S}^{\delta}$ therefore extends over a Zariski-open subset of the variety. 
Now for each $\alpha \in \chi_{S, \delta}^{k}$, we define a Zariski-open set

$$
U_{\alpha}^{\prime}=\bigcap_{\{p, q, r\} \in \alpha}\left\{\Delta_{p q, r} \neq 0\right\} \cap U_{\alpha} \subset \mathfrak{M}_{0, S}^{\delta}
$$

where the intersection is over all sets of (ordered) internal triangles $\{p, q, r\} \in \alpha$ and $\Delta_{p q, r}$ is defined by (2.29). It follows from (2.29) that $D_{\alpha} \subset U_{\alpha}^{\prime}$.

Proposition 2.22. - Let $\alpha \in \chi_{S, \delta}^{\ell}$ denote any ordered triangulation of the $n$-gon $(S, \delta)$. The set of vertex coordinates $\left\{x_{1}^{\alpha}, \ldots, x_{\ell}^{\alpha}\right\}$ defines a map

$$
\left(x_{1}^{\alpha}, \ldots, x_{\ell}^{\alpha}\right): U_{\alpha}^{\prime} \longrightarrow \mathbb{A}^{\ell}
$$

which is étale. It therefore defines a system of coordinates on $U_{\alpha}^{\prime}(\mathbb{R})$ or $U_{\alpha}^{\prime}(\mathbb{C})$.

Proof. - By iterating the decomposition (2.31), we obtain an embedding

$$
U_{\alpha} \hookrightarrow \prod_{i=1}^{N} U_{\alpha_{i}},
$$

where each $\alpha_{i}$ is a triangulation of a $k_{i}$-gon which cannot be decomposed any further. We can assume that each decomposition is strict, i.e., $k_{i} \geq 5$ for each $i$.

Two cases can occur. If there are no internal triangles, then necessarily $k_{i}=5$, and we can assume that $\alpha_{i}=\{\{1,3\},\{1,4\}\}$, so that $x_{1}^{\alpha_{i}}=u_{13}$, and $x_{2}^{\alpha_{i}}=u_{14}$. In that case, $(2.10)$ gives:

$$
u_{25}=1-u_{13} u_{14}, \quad u_{24} u_{25}=1-u_{13}, \quad \text { and } \quad u_{35} u_{25}=1-u_{14} .
$$

The variables on the left are invertible on $U_{\alpha_{i}}=\left\{u_{25} \neq 0\right\} \cap\left\{u_{24} \neq 0\right\} \cap\left\{u_{35} \neq 0\right\}$, so it follows that $x_{1}^{\alpha_{i}}, x_{2}^{\alpha_{i}}$ is a coordinate system on this open set, i.e., the map

$$
\left(x_{1}^{\alpha_{i}}, x_{2}^{\alpha_{i}}\right): U_{\alpha_{i}} \longrightarrow \mathbb{A}^{2}
$$

is certainly étale. On the other hand, if there is an internal triangle $\{p, q, r\}$, and the $k_{i}$-gon cannot be decomposed any further, then we are in the situation corresponding to $\mathfrak{M}_{0,6}$ pictured above (Fig. 6, middle). By symmetry, we can assume that $x_{1}^{\alpha_{i}}=u_{13}, x_{2}^{\alpha_{i}}=u_{15}$, and $x_{3}^{\alpha_{i}}=u_{35}$. By Equation (2.28), the variable $\pi_{r}=u_{25}$ depends quadratically on $x_{1}^{\alpha_{i}}, x_{2}^{\alpha_{i}}, x_{3}^{\alpha_{i}}$. It follows from the triangle lemma (2.17) and the definition of $U_{\alpha_{i}}^{\prime}$ that the map

$$
\left(x_{1}^{\alpha_{i}}, x_{2}^{\alpha_{i}}, x_{3}^{\alpha_{i}}\right): U_{\alpha_{i}}^{\prime} \longrightarrow \mathbb{A}^{3}
$$

is étale and two to one. This is because all remaining dihedral coordinates $u_{i j}$ are uniquely determined by $x_{1}^{\alpha_{i}}=u_{15}, \pi_{r}=u_{25}$, and $x_{3}^{\alpha_{i}}=u_{35}$ by applying the relation (2.10) and inverting coordinates which do not vanish on $U_{\alpha_{i}}^{\prime}$ in much the same way as above. From (2.34) we obtain an embedding $U_{\alpha}^{\prime} \hookrightarrow \prod_{i=1}^{N} U_{\alpha_{i}}^{\prime}$, which in turn gives rise to a commutative diagram

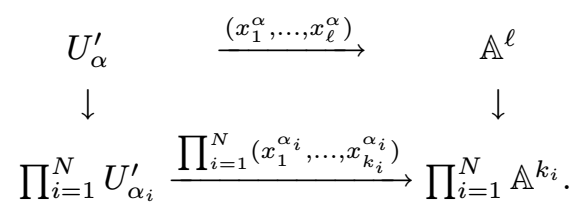

The vertical maps on the left and on the right are diagonal maps. We have shown that the horizontal map along the bottom is étale. It follows that the horizontal map along the top is étale, which completes the proof. 
If the triangulation $\alpha$ contains no internal triangles, then $U_{\alpha}^{\prime}=U_{\alpha}$, and the functions $x_{1}^{\alpha}, \ldots, x_{\ell}^{\alpha}$ give an isomorphism of $\mathfrak{M}_{0, S}$ with a Zariski open subset of $\mathbb{A}^{\ell}$.

COROLlary 2.23. - Let $\alpha \in \chi_{S, \delta}^{\ell}$, such that $\alpha$ has no internal triangles (and therefore two free vertices). Then the set $x_{1}^{\alpha}, \ldots, x_{\ell}^{\alpha}$ is a system of coordinates everywhere on $\mathfrak{M}_{0, S}$, and every cross-ratio $u_{i j}$ is a rational function of the $x_{i}^{\alpha}$.

We could also define $\mathfrak{M}_{0, S}^{\delta}$ using the set of equations (2.27). One can verify that if $\alpha \in \chi_{S, \delta}^{\ell}$ has no internal triangles, then all dihedral coordinates can be expressed in terms of the vertex coordinates $\left\{x_{i}^{\alpha}\right\}$ by repeatedly applying the butterfly lemma.

Lemma 2.24. - The sets $U_{\alpha}^{\prime}$, for $\alpha \in \chi_{S, \delta}^{\ell}$, cover $\mathfrak{M}_{0, S}^{\delta}$.

Proof. - For each partial decomposition $\beta \in \chi_{S, \delta}^{k}$, set

$$
N_{\beta}=\left\{u_{i j}=0 \text { for all }\{i, j\} \in \beta\right\} \cap\left\{u_{p q} \neq 0 \text { for all }\{p, q\} \notin \beta\right\},
$$

which is an open subset of $D_{\beta}=\left\{u_{i j}=0\right.$ for all $\left.\{i, j\} \in \beta\right\}$. It follows immediately from this definition that $\mathfrak{M}_{0, S}^{\delta}$ decomposes as a disjoint union:

$$
\mathfrak{M}_{0, S}^{\delta}=\mathfrak{M}_{0, S} \cup \bigcup_{k=1}^{\ell} \bigcup_{\beta \in \chi_{S, \delta}^{k}} N_{\beta}
$$

Let $\beta \in \chi_{S, \delta}^{k}$ denote any partial decomposition of the $n$-gon $(S, \delta)$. By adding chords, we can find a full triangulation $\alpha \in \chi_{S, \delta}^{\ell}$ which contains $\beta$, without creating any new internal triangles in $\alpha$. It follows from (2.29) and Lemma 2.17 that $N_{\beta} \subset U_{\alpha}^{\prime}$. Note that if $\beta$ is the empty triangulation, then $\mathfrak{M}_{0, S} \subset U_{\alpha}^{\prime}$ for any $\alpha$ which has no internal triangles. The decomposition (2.35) then implies that

$$
\mathfrak{M}_{0, S}^{\delta} \subset \bigcup_{\alpha \in \chi_{S, \delta}^{\ell}} U_{\alpha}^{\prime}
$$

In this manner, we obtain a second proof of the following theorem:

Theorem 2.25. - The affine varieties $\mathfrak{M}_{0, S}^{\delta}(\mathbb{R})$ or $\mathfrak{M}_{0, S}^{\delta}(\mathbb{C})$ are smooth and irreducible, and the divisors $D_{i j}$, for $\{i, j\} \in \chi_{S, \delta}$, are smooth and normal crossing.

Proof. - Let $\alpha \in \chi_{S, \delta}^{\ell}$. Proposition 2.22 states that the vertex coordinates $x_{1}^{\alpha}, \ldots, x_{\ell}^{\alpha}$ corresponding to $\alpha$ define an étale map $U_{\alpha}^{\prime} \rightarrow \mathbb{A}^{\ell}$. The image of $\mathfrak{M}_{0, S} \cap U_{\alpha}^{\prime}$ in $U_{\alpha}^{\prime}$ is precisely the complement of the normal crossing divisor

$$
x_{1}^{\alpha} \ldots x_{\ell}^{\alpha}=0
$$

(see Fig. 8). The theorem follows since the sets $U_{\alpha}^{\prime}$ cover $\mathfrak{M}_{0, S}^{\delta}$. 


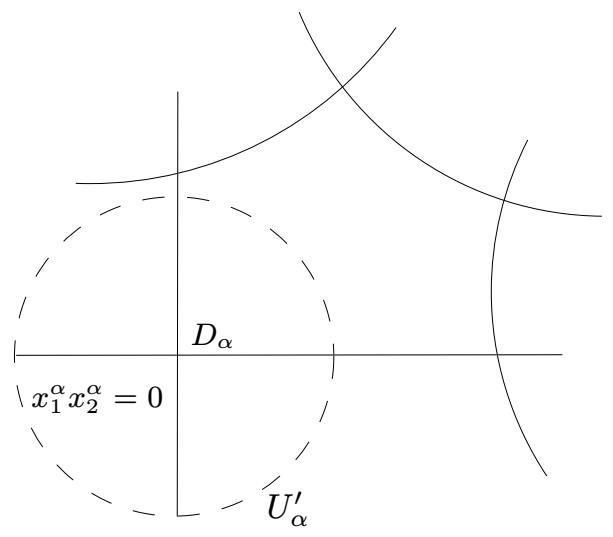

Figure 8 . The covering of $\mathfrak{M}_{0,5}^{\delta}$. To each vertex $\alpha \in \chi_{S, \delta}^{\ell}$, there is an open set $U_{\alpha}^{\prime}$ on which the set of vertex coordinates $\left\{x_{i}^{\alpha}\right\}$ cross normally. The sets $U_{\alpha}^{\prime}$ form a covering, which gives a second proof that the divisors $D_{i j}$ are smooth and normal crossing.

\subsection{The real moduli space $\mathfrak{M}_{0, S}^{\delta}(\mathbb{R})$}

Consider the moduli space of projective circles with $n$ ordered marked points:

$$
\mathfrak{M}_{0, S}(\mathbb{R})=\mathrm{PSL}_{2}(\mathbb{R}) \backslash \mathbb{P}^{1}(\mathbb{R})_{*}^{n} .
$$

The space $\mathfrak{M}_{0, S}(\mathbb{R})$ is not connected, but is a disjoint union of open cells which we define as follows. First, we fix a dihedral structure $\delta$ on $S$, which defines a set of dihedral coordinates $u_{i j}$ for $\{i, j\} \in \chi_{S, \delta}$. Let

$$
\bar{X}_{S, \delta}=\left\{u_{i j} \geq 0:\{i, j\} \in \chi_{S, \delta}\right\} \subset \mathfrak{M}_{0, S}^{\delta}(\mathbb{R}) .
$$

By (2.10), $\bar{X}_{S, \delta}$ is also defined by the equations $0 \leq u_{i j} \leq 1$ for all $\{i, j\} \in \chi_{S, \delta}$, and is therefore compact. We define the open cell $X_{S, \delta}$ to be the interior of $\bar{X}_{S, \delta}$ :

$$
X_{S, \delta}=\bar{X}_{S, \delta} \cap \mathfrak{M}_{0, S}=\left\{u_{i j}>0:\{i, j\} \in \chi_{S, \delta}\right\} .
$$

The sets $X_{S, \delta}$ and $\bar{X}_{S, \delta}$ are clearly preserved by the dihedral symmetries of $\delta$, so there is an action of the dihedral group $D_{2 n} \times \bar{X}_{S, \delta} \rightarrow \bar{X}_{S, \delta}$. Using explicit simplicial coordinates (2.9), one checks that the open set $X_{S, \delta}$ is homeomorphic to the simplex

$$
X_{S, \delta} \cong\left\{\left(t_{1}, \ldots, t_{\ell}\right) \quad: \quad 0<t_{1}<\cdots<t_{\ell}<1\right\} .
$$

It follows that $X_{S, \delta}$ is contractible, and moreover, that $X_{S, \delta}$ is a connected component of $\mathfrak{M}_{0, S}(\mathbb{R})$. After changing to cubical coordinates, we see that $X_{S, \delta}$ is the unit hypercube $\left\{\left(x_{1}, \ldots, x_{\ell}\right): x_{i} \in(0,1)\right\}=(0,1)^{\ell}$, which explains the nomenclature of each coordinate system (Fig. 9).

Each cell $X_{S, \delta}$ consists of the set of points $s_{1}, \ldots, s_{n} \in \mathbb{P}^{1}(\mathbb{R})$ such that $s_{1}, \ldots, s_{n}$ are in the dihedral order determined by $\delta$. Two components $X_{S, \delta}, X_{S, \delta^{\prime}}$ are disjoint if $\delta$ and $\delta^{\prime}$ are distinct, and the set of dihedral structures are permuted transitively by the symmetric group $\mathfrak{S}_{n}$. This implies the following tiling lemma. Devadoss has studied the exact gluing relations between the cells $X_{S, \delta}$ in this tiling [15]. 
Lemma 2.26. - The space $\mathfrak{M}_{0, S}(\mathbb{R})$ is the disjoint union of the $n ! / 2 n$ open cells $X_{S, \delta}$, as $\delta \in \mathfrak{S}_{n} / D_{2 n}$ ranges over the set of all dihedral structures on $S$.

It is now clear that the choice of a dihedral structure $\delta$ on $S$ is equivalent to the choice of a fundamental cell $X_{S, \delta} \in \pi_{0}\left(\mathfrak{M}_{0, S}(\mathbb{R})\right) \cong \mathfrak{S}_{n} / D_{2 n}$. The set of dihedral coordinates $u_{i j}$ corresponding to $\delta$ can be regarded as a natural set of functions which is stable under the action of the symmetry group of $X_{S, \delta}$.
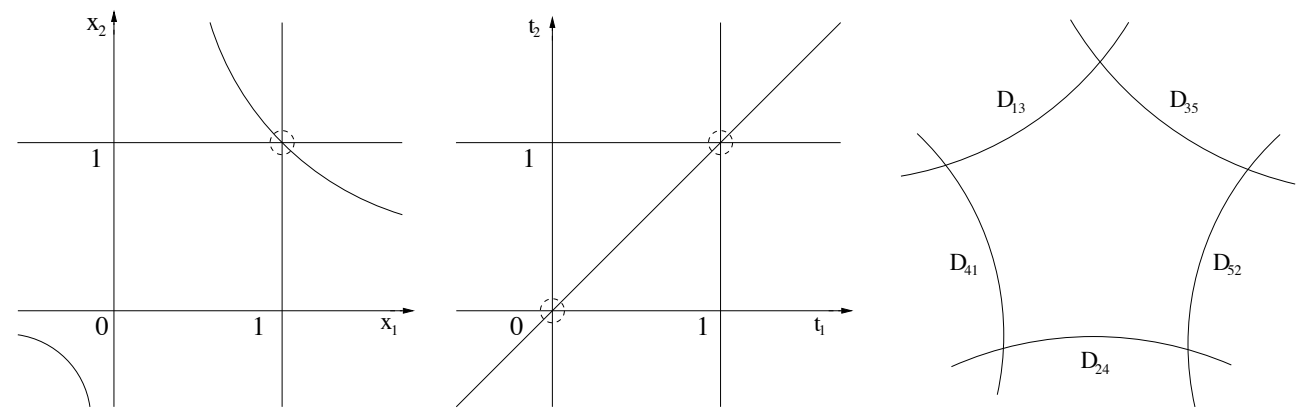

Figure 9. The set of real points $\mathfrak{M}_{0,5}(\mathbb{R})$ in cubical (left), simplicial (middle), and dihedral coordinates (right). The dotted circles denote points which are blown up when passing to dihedral coordinates. There are 5!/10 $=12$ regions $X_{S, \delta}$ when $|S|=5$.

Definition 2.27. - For each chord $\{i, j\} \in \chi_{S, \delta}$, we define the face $F_{i j}\left(\bar{X}_{S, \delta}\right)$ of $\bar{X}_{S, \delta}$ to be the closed subset $F_{i j}\left(\bar{X}_{S, \delta}\right)=D_{i j} \cap \bar{X}_{S, \delta} \subset \mathfrak{M}_{0, S}^{\delta}(\mathbb{R})$. Likewise, for each $\alpha \in \chi_{S, \delta}^{k}$, we define the codimension- $k$ face of $\bar{X}_{S, \delta}$ to be $F_{\alpha}\left(\bar{X}_{S, \delta}\right)=D_{\alpha} \cap \bar{X}_{S, \delta}$.

It follows from Lemma 2.5 that

$$
F_{i j}\left(\bar{X}_{S, \delta}\right) \cong \bar{X}_{T_{1} \cup\{e\}, \delta_{1}} \times \bar{X}_{T_{2} \cup\{e\}, \delta_{2}},
$$

where $T_{1} \cup T_{2}$ is the partition of (the set of edges) $S$ corresponding to the chord $e=\{i, j\}$. By Equation (2.18), each codimension- $k$ face $F_{\alpha}\left(\bar{X}_{S, \delta}\right)$ is a product

$$
F_{\alpha}\left(\bar{X}_{S, \delta}\right) \cong \prod_{m=1}^{k+1} \bar{X}_{S_{m}, \delta_{m}} .
$$

By repeatedly taking boundaries we obtain a stratification:

$$
\bar{X}_{S, \delta} \supseteq \partial \bar{X}_{S, \delta} \supseteq \partial^{2} \bar{X}_{S, \delta} \supseteq \cdots \supseteq \partial^{\ell} \bar{X}_{S, \delta},
$$

where the codimension- $k$ boundary of $\bar{X}_{S, \delta}$ is the union of its codimension- $k$ faces:

$$
\partial^{k} \bar{X}_{S, \delta}=\bigcup_{\alpha \in \chi_{S, \delta}^{k}} F_{\alpha}\left(\bar{X}_{S, \delta}\right) \quad \text { for } 1 \leq k \leq \ell .
$$

For each $n \geq 4$, the associahedron $K_{n-1}$, or Stasheff polytope [48], is a convex polytope of dimension $n-3$ whose codimension- $k$ faces are indexed by the partially ordered set of compatible bracketings on a set of $n-1$ elements. 
COROLlaRY 2.28. - The lattice of faces $\bar{X}_{S, \delta}$ is combinatorially equivalent to the associahedron $K_{n-1}$.

Proof. - The set of all codimension- $k$ faces $F_{\alpha}\left(\bar{X}_{S, \delta}\right)$ is indexed by $k$-triangulations of a regular $n$-gon, and the inclusion of one face in another is given by removing a chord. By taking the dual graph of a partial triangulation of an $n$-gon we obtain a planar tree. If we fix an edge $s_{1}$ of $S$, then each such tree is rooted, and defines, in a standard way, a bracketing of the ordered set $\left\{s_{2}, \ldots, s_{n}\right\}$ (fig. 11). We obtain in this way a bijection between faces of $\bar{X}_{S, \delta}$ and bracketings on a set of $n-1$ elements (this is beautifully illustrated in [16]).

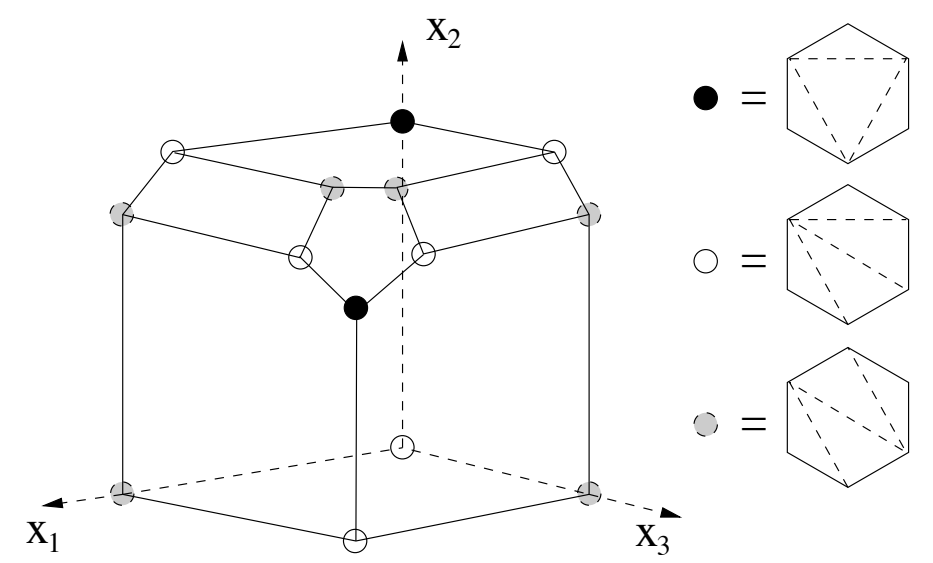

FIGURE 10. The associahedron or Stasheff polytope $\bar{X}_{6, \delta}=K_{5}$ in $\mathfrak{M}_{0,6}^{\delta}(\mathbb{R})$ obtained by truncating the unit cube in $\mathbb{R}^{3}$, or blowing up along $x_{1}=x_{2}=x_{3}=1$, and then $x_{1}=x_{2}=1$ and $x_{2}=x_{3}=1$. It has six faces $F_{13}, F_{24}, F_{35}, F_{46}, F_{51}, F_{62}$ which are pentagons $\bar{X}_{5, \delta}$, and three faces $F_{14}, F_{25}, F_{36}$ which are quadrilaterals $\bar{X}_{4, \delta_{1}} \times \bar{X}_{4, \delta_{1}}$. These are permuted by the group $D_{12}$. There are three types of vertices corresponding to three kinds of triangulation of a hexagon. The vertex coordinates defined in $\$ 2.6$ for each triangulation provide local affine charts in the neighbourhood of each vertex.

Since each face $F_{\alpha}$ is contractible, we can view $\bar{X}_{S, \delta}$ in the coordinates $u_{i j}$ as a dihedrallysymmetric algebraic model of the associahedron $K_{n-1}$. The fact that the divisors $D_{i j}$ cross normally implies that the associahedron is a simple polytope, i.e., each vertex is the intersection of exactly $\ell$ distinct faces.

REMARK 2.29. - As remarked earlier, $\mathfrak{M}_{0, S}^{\delta}$ can be obtained by blowing up a set of divisors bounding $X_{S, \delta}$. Since the operation of blowing up is non-commutative, we have to specify that the blow-ups occur along subvarieties in increasing order of dimension. A useful intuitive picture of the polytopes $\bar{X}_{S, \delta}$ can be obtained by blowing up the unit hypercube $[0,1]^{\ell}$ along the divisors $x_{i}=\cdots=x_{j}=1$ for $1 \leq i<j \leq \ell$. The set of real points in the blow-up can be visualised by truncating the unit hypercube along the hypersurfaces $x_{i} \ldots x_{j}=1-\varepsilon$, where $i<j$ for some fixed $\varepsilon>0$ which is sufficiently small (see fig. 10). Alternatively, one could truncate the simplicial model of $X_{S, \delta}$ to obtain another explicit construction of $K_{n-1}$ (see [16]). This involves a greater number of truncations, however. 


\subsection{The compactification $\overline{\mathfrak{M}}_{0, S}$ and its divisors at infinity}

The set of all cross-ratios defines an embedding:

$$
\{[i j \mid k l]\}: \mathfrak{M}_{0, S} \longrightarrow \mathfrak{M}_{0,4}^{\left(\begin{array}{c}
n \\
4
\end{array}\right)} \cong\left(\mathbb{P}^{1} \backslash\{0,1, \infty\}\right)^{\left(\begin{array}{c}
n \\
4
\end{array}\right)} .
$$

The coordinates $\{[i j \mid k l]\}$ satisfy identities (2.1) and (2.2). These identities define a projective scheme we denote

$$
\overline{\mathfrak{M}}_{0, S} \subset\left(\mathbb{P}^{1}\right)^{\left(\begin{array}{c}
n \\
4
\end{array}\right),}
$$

which is defined over $\mathbb{Z}$. This representation of $\overline{\mathfrak{M}}_{0, S}$ is degenerate since some coordinates are the same, but it is clearly invariant under the action of the symmetric group. For every cross-ratio $[i j \mid k l]$, with $i, j, k, l \in S$, there is a divisor

$$
D_{[i j \mid k l]}=\{[i j \mid k l]=0\} \subset \overline{\mathfrak{M}}_{0, S} .
$$

It follows from simple properties of the cross-ratio (2.1) that

$$
D_{[i j \mid k l]} \cap D_{[i k \mid j l]}=\varnothing, \text { and } D_{[i j \mid k l]} \cap D_{[i j \mid l k]}=\varnothing,
$$

and $D_{[i j \mid k l]}=D_{[k l \mid i j]}=D_{[j i \mid l k]}=D_{[l k \mid j i]}$. For each dihedral structure $\delta$ on $S$, define an open subset $U^{\delta} \subset \overline{\mathfrak{M}}_{0, S}$ by:

$$
U^{\delta}=\{[i j \mid k l] \neq 0 \text { for all } i, j, k, l \text { in dihedral order } \delta\} .
$$

Thus $U^{\delta}=\overline{\mathfrak{M}}_{0, S} \backslash Z_{\delta}$, where $Z_{\delta}$ is the union of divisors $D_{[i j \mid k l]}$ for every quadruple $(i, j, k, l)$ in dihedral order $\delta$.

Lemma 2.30. - There is an isomorphism $j_{\delta}: \mathfrak{M}_{0, S}^{\delta} \stackrel{\sim}{\longrightarrow} U^{\delta}$.

Proof. - By the relations (2.1), a set of coordinates on $U_{\delta}$ is given by the cross-ratios $[i j \mid l k]$ for all $(i, j, k, l)$ in dihedral order $\delta$. The result follows from the definition (2.12) of $\mathfrak{M}_{0, S}^{\delta}$.

For example, $\overline{\mathfrak{M}}_{0,4}$ is defined by 24 cross-ratios $[i j \mid k l]$, with $\{i, j, k, l\}=\{1,2,3,4\}$, each of which is equal to one of the 6 anharmonic ratios $\left\{x, 1-x, \frac{1}{x}, \frac{x-1}{x}, \frac{x}{x-1}, \frac{1}{1-x}\right\}$, where $x=\left[\begin{array}{ll}1 & 2 \mid 4\end{array}\right]$. Thus $\overline{\mathfrak{M}}_{0,4}$ is isomorphic to $\mathbb{P}^{1}$. The three distinct dihedral structures on a set with four elements give three embeddings of $\mathfrak{M}_{0,4}^{\delta} \cong \mathbb{A}^{1} \hookrightarrow \mathbb{P}^{1}$, whose images are $\mathbb{P}^{1} \backslash\{0\}$, $\mathbb{P}^{1} \backslash\{1\}$, and $\mathbb{P}^{1} \backslash\{\infty\}$.

Lemma 2.31. - The compactification $\overline{\mathfrak{M}}_{0, S}$ is covered by affine charts $\mathfrak{M}_{0, S}^{\delta}$, as $\delta$ ranges over the set of all dihedral structures on $S$ :

$$
\overline{\mathfrak{M}}_{0, S}=\bigcup_{\delta \in \mathfrak{S}_{n} / D_{2 n}} j_{\delta}\left(\mathfrak{M}_{0, S}^{\delta}\right)
$$

Proof. - To show that the sets $U^{\delta}=j_{\delta}\left(\mathfrak{M}_{0, S}^{\delta}\right)$ form an open affine covering of $\overline{\mathfrak{M}}_{0, S}$ is equivalent to proving that $\bigcap_{\delta} Z_{\delta}=\varnothing$, where $\delta$ ranges over all $N=n ! / 2 n$ dihedral structures on $S$. But

$$
\bigcap_{\delta} Z_{\delta}=\bigcap_{\delta} \bigcup_{(i, j, k, l) \in \delta^{4}} D_{[i j \mid k l]}=\bigcup_{\left(i_{m}, j_{m}, k_{m}, l_{m}\right) \in \delta_{m}^{4}}\left(D_{\left[i_{1} j_{1} \mid k_{1} l_{1}\right]} \cap \cdots \cap D_{\left[i_{N} j_{N} \mid k_{N} l_{N}\right]}\right),
$$


where $\delta^{4}$ denotes the set of all 4-tuples $(i, j, k, l)$ which are in dihedral order $\delta$, and where $\delta_{1}, \ldots, \delta_{N}$ are representatives of every dihedral structure on $S$. It suffices to show that for every $\left(i_{1}, j_{1}, k_{1}, l_{1}\right) \in \delta_{1}^{4}, \ldots,\left(i_{N}, j_{N}, k_{N}, l_{N}\right) \in \delta_{N}^{4}$, we have

$$
D_{\left[i_{1} j_{1} \mid k_{1} l_{1}\right]} \cap \cdots \cap D_{\left[i_{N} j_{N} \mid k_{N} l_{N}\right]}=\varnothing .
$$

Since for all $n \geq 4$,

$$
\#\{\text { dihedral structures }\}=N=\frac{n !}{2 n}>\left(\begin{array}{l}
n \\
4
\end{array}\right)=\#\{4 \text {-tuples }\{i, j, k, l\}\},
$$

there must, by the pigeonhole principle, be at least two sets of indices $\left\{i_{p}, j_{p}, k_{p}, l_{p}\right\}$, and $\left\{i_{q}, j_{q}, k_{q}, l_{q}\right\}$ in (2.44) which coincide. But since they necessarily occur in distinct dihedral orders, we have by (2.42) that $D_{\left[i_{p} j_{p} \mid k_{p} l_{p}\right]} \cap D_{\left[i_{q} j_{q} \mid k_{q} l_{q}\right]}=\varnothing$, which proves (2.44).

Theorem (2.25) implies the following corollary.

Corollary 2.32. - $\overline{\mathfrak{M}}_{0, S}$ is smooth and $\overline{\mathfrak{M}}_{0, S} \backslash \mathfrak{M}_{0, S}$ is a normal crossing divisor.

The irreducible components at infinity of $\overline{\mathfrak{M}}_{0, S} \backslash \mathfrak{M}_{0, S}$ can be described as follows.

Lemma 2.33. - Let $\delta$ denote a dihedral structure on $S$, and let $\{p, q\} \in \chi_{S, \delta}$. The chord $\{p, q\}$ partitions the set $S$, viewed as edges of the $n$-gon $(S, \delta)$, into two sets $P_{1} \cup P_{2}$. Then the divisor $j_{\delta}\left(D_{p q}\right) \subset j_{\delta}\left(\mathfrak{M}_{0, S}^{\delta}\right)$ is determined by the equations $[i j \mid k l]=0$ for all distinct indices $i, j, k, l$ such that

$$
\begin{aligned}
& \{i, k\} \subset P_{1} \text { and }\{j, l\} \subset P_{2}, \\
\text { or } \quad & \{j, l\} \subset P_{1} \text { and }\{i, k\} \subset P_{2} .
\end{aligned}
$$

Proof. - On the chart $j_{\delta}\left(\mathfrak{M}_{0, S}^{\delta}\right)$, these equations imply in particular that $u_{p q}=0$, and therefore determine the divisor $j_{\delta}\left(D_{p q}\right)$. That the remaining cross-ratios also vanish on $j_{\delta}\left(D_{p q}\right)$ follows from Lemma 2.2 .

It follows that two divisors $j_{\delta_{1}}\left(D_{1}\right)$ and $j_{\delta_{2}}\left(D_{2}\right)$ coincide on $j_{\delta_{1}}\left(\mathfrak{M}_{0, S}^{\delta_{1}}\right) \cap j_{\delta_{2}}\left(\mathfrak{M}_{0, S}^{\delta_{2}}\right)$ if and only if the corresponding partitions of $S$ agree.

Definition 2.34. - A partition $P_{1} \cup P_{2}=S$ is stable if $\left|P_{1}\right| \geq 2$ and $\left|P_{2}\right| \geq 2$. A dihedral structure $\delta$ on $S$ is compatible with $P_{1} \cup P_{2}$ if the elements of each set $P_{1}$ and $P_{2}$ are consecutive with respect to $\delta$. An irreducible divisor $D \subset \overline{\mathfrak{M}}_{0, S} \backslash \mathfrak{M}_{0, S}$ is said to be at finite distance with respect to a dihedral structure $\delta$, if $D \cap j_{\delta}\left(\mathfrak{M}_{0, S}^{\delta}\right) \neq \varnothing$.

Proposition 2.35. - There is a bijection between the irreducible components of the divisors at infinity of $\overline{\mathfrak{M}}_{0, S} \backslash \mathfrak{M}_{0, S}$, and stable partitions $S=P_{1} \cup P_{2}$. The component $D$ corresponding to this partition is canonically isomorphic to

$$
\overline{\mathfrak{M}}_{0, P_{1} \cup\{e\}} \times \overline{\mathfrak{M}}_{0, P_{2} \cup\{e\}},
$$

where e is a symbol. A divisor is at finite distance with respect to a dihedral structure $\delta$ if and only if $\delta$ is compatible with the corresponding partition of $S$. 
Proof. - The bijection between stable partitions and divisors follows immediately from the previous remarks and the covering (2.43). The last statement of the proposition holds by definition. It remains to prove the decomposition. Suppose that we are given a stable partition $S=P_{1} \cup P_{2}$, and let $D$ denote the corresponding divisor. Note that a dihedral structure $\delta$ on $S$, compatible with $P_{1} \cup P_{2}$, induces dihedral structures $\delta_{1}, \delta_{2}$ on the sets $P_{1} \cup$ $\{e\}$ and $P_{2} \cup\{e\}$ (compare Fig. 3), and every pair of $\delta_{1}, \delta_{2}$ arises in this way. It follows from Lemma 2.6 that:

$$
D \cap j_{\delta}\left(\mathfrak{M}_{0, S}^{\delta}\right) \cong \begin{cases}j_{\delta}\left(\mathfrak{M}_{0, P_{1} \cup\{e\}}^{\delta_{1}} \times \mathfrak{M}_{0, P_{2} \cup\{e\}}^{\delta_{2}}\right) & \text { if } \delta \text { is compatible with } P_{1} \cup P_{2}, \\ \varnothing & \text { otherwise. }\end{cases}
$$

If we identify $j_{\delta}\left(\mathfrak{M}_{0, P_{1} \cup\{e\}}^{\delta_{1}} \times \mathfrak{M}_{0, P_{2} \cup\{e\}}^{\delta_{2}}\right)$ with $j_{\delta_{1}}\left(\mathfrak{M}_{0, P_{1} \cup\{e\}}^{\delta_{1}}\right) \times j_{\delta_{2}}\left(\mathfrak{M}_{0, P_{2} \cup\{e\}}^{\delta_{2}}\right)$ in $\overline{\mathfrak{M}}_{0, P_{1} \cup\{e\}} \times \overline{\mathfrak{M}}_{0, P_{1} \cup\{e\}}$, we obtain:

$$
\begin{aligned}
D & =D \cap \overline{\mathfrak{M}}_{0, S}=D \cap \bigcup_{\delta} j_{\delta}\left(\mathfrak{M}_{0, S}^{\delta}\right) \cong \bigcup_{\delta_{1}, \delta_{2}} j_{\delta}\left(\mathfrak{M}_{0, P_{1} \cup\{e\}}^{\delta_{1}} \times \mathfrak{M}_{0, P_{2} \cup\{e\}}^{\delta_{2}}\right) \\
& \cong \bigcup_{\delta_{1}} j_{\delta_{1}}\left(\mathfrak{M}_{0, P_{1} \cup\{e\}}^{\delta_{1}}\right) \times \bigcup_{\delta_{2}} j_{\delta_{2}}\left(\mathfrak{M}_{0, P_{2} \cup\{e\}}^{\delta_{2}}\right)=\overline{\mathfrak{M}}_{0, P_{1} \cup\{e\}} \times \overline{\mathfrak{M}}_{0, P_{2} \cup\{e\}} .
\end{aligned}
$$

We introduce the following notation. Let $D$ denote the divisor given by a stable partition $S=P_{1} \cup P_{2}$. Then for any pair of indices $i, j \in S$, we set

$$
\mathbb{I}_{D}(i, j)=\mathbb{I}\left(\{i, j\} \subset P^{1}\right)+\mathbb{I}\left(\{i, j\} \subset P^{2}\right),
$$

where $\mathbb{I}(A \subset B)$ is the indicator function which takes the value 1 if a set $A$ is contained in $B$, and 0 otherwise.

Corollary 2.36. - Let $D$ denote the divisor corresponding to the stable partition $S=P_{1} \cup P_{2}$. The order of vanishing of any cross-ratio along $D$ is given by:

$$
\operatorname{ord}_{D}[i j \mid k l]=\frac{1}{2}\left[\mathbb{I}_{D}(i, k)+\mathbb{I}_{D}(j, l)-\mathbb{I}_{D}(i, l)-\mathbb{I}_{D}(j, k)\right] .
$$

Proof. - The formula is invariant under the action of $\mathfrak{S}(S)$ on divisors and crossratios. We can therefore fix a dihedral structure $\delta$ on $S$ and assume that $D=D_{2 a}$, where $a \in\{4, \ldots, n\}$. The formula is also compatible with (2.1) and additive with respect to (2.2). By Lemma 2.2, it therefore suffices to verify the formula for $[p p+1 \mid q+1 q]=u_{p q}$, where $\{p, q\} \in \chi_{S, \delta}$. It follows from (2.10) that $\operatorname{ord}_{D_{2 a}} u_{p q}$ is 1 if $\{p, q\}=\{2, a\}$ and is 0 otherwise. The partition corresponding to $D_{2 a}$ is $\{3,4, \ldots, a\} \cup\{a+1, \ldots, n, 1,2\}$, and it is easy to check that the formula holds in this case.

A stable partition $S=P_{1} \cup P_{2}$ is conveniently represented as the union of two circles, joined at a point $e$, with marked points corresponding to $P_{1}$ on the first circle, and those corresponding to $P_{2}$ on the other. Taking iterated intersections of divisors, one obtains bubble diagrams (fig. 11) [16]. Such a diagram defines a tree, and one retrieves the standard combinatorial description of strata in $\overline{\mathfrak{M}}_{0, S}$. If we take the dual graph, we obtain a partial decomposition of a polygon. Note that we can find dihedral structures $\delta$ for which the labellings of the outer edges are in dihedral order with respect to $\delta$. In this way, any bubble diagram corresponds to an intersection of divisors at finite distance on a certain number of affine pieces $\mathfrak{M}_{0, S}^{\delta}$ in $\overline{\mathfrak{M}}_{0, S}$. 

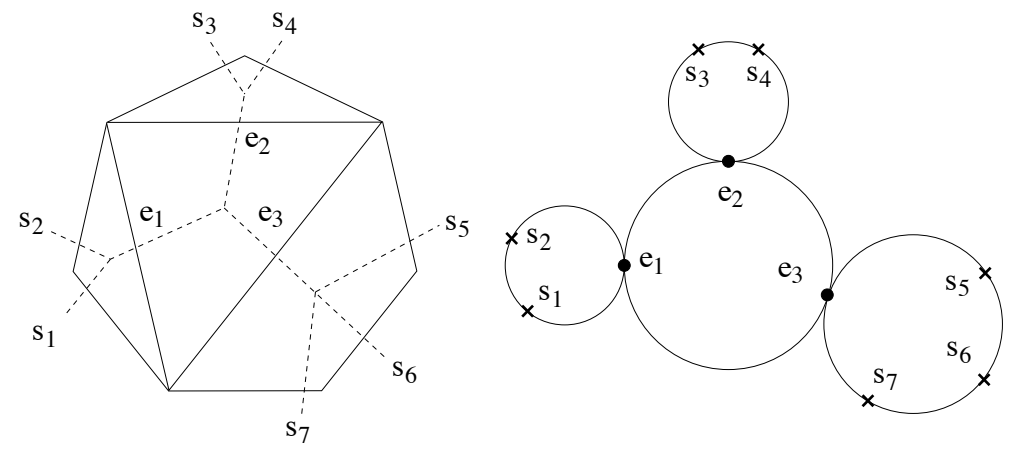

Figure 11. A partial decomposition of a heptagon (left), its dual graph (dotted lines), and the corresponding bubble diagram (right). If the tree is rooted at $s_{1}$, this corresponds to the bracketing $\left(s_{2},\left(\left(s_{3}, s_{4}\right),\left(s_{5}, s_{6}, s_{7}\right)\right)\right)$.

\subsection{Comparison with the Deligne-Mumford-Knudsen construction}

It remains to verify that our definition of $\overline{\mathfrak{M}}_{0, S}$ coincides with the construction $\overline{\mathfrak{M}}_{0, S}^{\mathrm{DMK}}$ due to Deligne-Mumford and Knudsen. The universal property for the latter implies that there is a morphism

$$
\overline{\mathfrak{M}}_{0, S} \longrightarrow \overline{\mathfrak{M}}_{0, S}^{\mathrm{DMK}} .
$$

One could probably check that this is an isomorphism using the combinatorial and geometric results proved for $\overline{\mathfrak{M}}_{0, S}$ above, but it would be preferable to prove the universal property directly for $\overline{\mathfrak{M}}_{0, S}$ using our definition in terms of the affine schemes $\mathfrak{M}_{0, S}^{\delta}$. We have not done this.

\subsection{Product maps}

The projection maps on $\mathfrak{M}_{0, S}$ defined above decrease the dimension by one. We will also need to consider various maps between products of moduli spaces $\mathfrak{M}_{0, T_{i}}$ which preserve the dimensions. These give rise to special coordinate systems on $\mathfrak{M}_{0, S}$ and will be used to define products on period integrals. Given two subsets $T_{1}, T_{2} \subset S$ such that $\left|T_{i}\right| \geq 4$, we consider maps of the form

$$
f=f_{T_{1}} \times f_{T_{2}}: \mathfrak{M}_{0, S} \longrightarrow \mathfrak{M}_{0, T_{1}} \times \mathfrak{M}_{0, T_{2}} .
$$

Such a map will be called a product map if

$$
\begin{aligned}
\left|T_{1} \cap T_{2}\right| & =3, \\
S & =T_{1} \cup T_{2} .
\end{aligned}
$$

In this case the dimensions on both sides of (2.46) are equal, since the equalities (2.47) imply that $|S|-3=\left|T_{1}\right|-3+\left|T_{2}\right|-3$. The map $f$ is an embedding, because we can place the three points in $T_{1} \cap T_{2}$ at 0,1 , and $\infty$, and each remaining marked point $s \in S$ is then uniquely determined by the map $f_{T_{i}}$ where $i \in\{1,2\}$ and $s \in T_{i}$. We can iterate this construction by further decomposing $T_{i}$ as a union of sets satisfying (2.47). Since the composition of two 
forgetful maps $f_{T}$ is itself a forgetful map, we obtain a family of subsets $T_{1}, \ldots, T_{k} \subset S$ such that $\left|T_{i}\right| \geq 4$, and a map

$$
f=\prod_{i=1}^{k} f_{T_{i}}: \mathfrak{M}_{0, S} \longrightarrow \prod_{i=1}^{k} \mathfrak{M}_{0, T_{i}} .
$$

This is an embedding by construction. The sets $T_{i}$ cover $S$, i.e., $S=\cup_{i=1}^{k} T_{i}$, and the equality of dimensions on the left and right hand sides of (2.48) implies that

$$
|S|+3(k-1)=\sum_{i=1}^{k}\left|T_{i}\right| .
$$

We can then regard $\mathfrak{M}_{0, S}$ as a dense open subscheme of $\prod_{i=1}^{k} \mathfrak{M}_{0, T_{i}}$, and we say that $f$ is a non-degenerate coordinate system on $\mathfrak{M}_{0, S}$. Any set of vertex coordinates $\left\{x_{i}^{\alpha}\right\}$ corresponding to a triangulation $\alpha \in \chi_{S, \delta}^{\ell}$, when $\alpha$ has no internal triangles, is an example of a non-degenerate coordinate system (this can be verified by induction). More precisely, if $x_{i}^{\alpha}=u_{p_{i} q_{i}}$, then we can cover $S$ with the sets $T_{i}=\left\{p_{i}, p_{i}+1, q_{i}, q_{i}+1\right\}$, and identify $\mathfrak{M}_{0, T_{i}}$ with $\mathbb{P}^{1} \backslash\{0,1, \infty\}$ using the coordinate $x_{i}^{\alpha}$, for $1 \leq i \leq \ell$. If, however, $\alpha$ has internal triangles, then the map $f$ corresponding to the set of vertex coordinates $x_{i}^{\alpha}$ is not an embedding, and therefore cannot be a non-degenerate coordinate system.

Let us fix a dihedral structure $\delta$ on $S$. This is equivalent, by $\S 2.7$, to choosing one of the open cells $X_{S, \delta}$ which covers $\mathfrak{M}_{0, S}(\mathbb{R})$. This induces a dihedral structure $\delta_{i}$ on each subset $T_{i} \subset S$, which in turn defines a fundamental cell $X_{T_{i}, \delta_{i}}$, for $1 \leq i \leq k$. By construction, $f_{T_{i}}\left(X_{S, \delta}\right) \subset X_{T_{i}, \delta_{i}}$, and therefore $f\left(X_{S, \delta}\right) \subset \prod_{i=1}^{k} X_{T_{i}, \delta_{i}}$. We define $G_{f}$ to be the set of all dihedral structures on $S$ which are compatible with the dihedral structures on each $T_{i}$ induced by $\delta$ : i.e.,

$$
G_{f}=\left\{\gamma \in \mathfrak{S}(S) / D_{2 n} \text { such that }\left.\gamma\right|_{T_{i}}=\delta_{i}\right\} .
$$

The precise relation between the domains $\prod_{i} X_{T_{i}, \delta_{i}}$ and $X_{S, \delta}$ is given by:

$$
f^{-1}\left(\prod_{i=1}^{k} X_{T_{i}, \delta_{i}}\right)=\coprod_{\gamma \in G_{f}} X_{S, \gamma} .
$$

Any point $x \in X_{S, \gamma}$ maps via $f$ into $\prod_{i} X_{T_{i}, \delta_{i}}$ if and only if $\left.\gamma\right|_{T_{i}}=\delta_{i}$. Identity (2.51) follows because the set of cells $X_{S, \gamma}$, for $\gamma \in \mathfrak{S}(S) / D_{2 n}$, covers $\mathfrak{M}_{0, S}(\mathbb{R})$ disjointly, by $\S 2.7$.

We consider two examples of such a map $f$, one for which $G_{f}$ is trivial, which gives rise to cubical coordinates, and the other for which $G_{f}$ is as large as possible, which defines simplicial coordinates. We will see later in $\S 7$ that these special cases give rise to the stuffle and shuffle relations for multiple zeta values, respectively.

We fix a dihedral structure $\delta$ on $S$, and write $S=\left\{s_{1}, \ldots, s_{n}\right\}$, as usual. Consider first of all the covering $S=\bigcup_{i=4}^{n} T_{i}$, where

$$
T_{i}=\left\{s_{2}, s_{3}, s_{i}, s_{i+1}\right\} \quad \text { for } \quad 4 \leq i \leq n,
$$

and all indices are modulo $n$, as usual. This defines a map

$$
f_{\square}: \mathfrak{M}_{0, S} \longrightarrow \prod_{i=4}^{n} \mathfrak{M}_{0, T_{i}},
$$


which satisfies condition (2.49). One verifies without difficulty that this is a non-degenerate coordinate system as defined above (or use the fact that $\left|T_{i} \cap T_{i+1}\right|=3$ for $4 \leq i \leq n-1$ ). We call $f_{\square}$ a system of cubical coordinates on $\mathfrak{M}_{0, S}$. In this case, $G_{f_{\square}}$ is trivial, since if $\gamma$ is a dihedral structure on $S$ compatible with all the dihedral structures $s_{2}<s_{3}<s_{i}<s_{i+1}$ on $T_{i}$ (or $s_{2}>s_{3}>s_{i}>s_{i+1}$ ), then we must have $s_{1}<\cdots<s_{n}<s_{1}\left(\right.$ or $\left.s_{1}>\cdots>s_{n}>s_{1}\right)$. Each moduli space $\mathfrak{M}_{0, T_{i}} \cong \mathfrak{M}_{0,4}$ is isomorphic to $\mathbb{P}^{1} \backslash\{0,1, \infty\}$ in six natural ways, corresponding to the six choices of cross-ratio on $\mathfrak{M}_{0,4}$. If we identify $\mathfrak{M}_{0, T_{i}}$ with $\mathbb{P}^{1} \backslash\{0,1, \infty\}$ using the coordinate $u_{2 i}=[23 \mid i+1 i]$, for $4 \leq i \leq n$, then, since $x_{i-3}=u_{2 i}$ by $(2.9)$, we retrieve the explicit cubical coordinates defined in $\S 2.1$. In other words, $(2.53)$ is just

$$
f_{\square}=\left(x_{1}, \ldots, x_{\ell}\right): \mathfrak{M}_{0, S} \rightarrow\left(\mathbb{A}^{1} \backslash\{0,1\}\right)^{\ell},
$$

and coincides with $(2.5)$. Each cell $X_{T_{i}, \delta_{i}}$ is the unit interval $(0,1)$ in these coordinates, and $X_{S, \delta}$ maps under $f_{\square}$ to $(0,1)^{\ell}$. In this case, Equation (2.51) simply states that a product of $\ell$ unit intervals is the unit $\ell$-dimensional hypercube.

Cubical coordinates come from a product map. If $k \geq 4$, we set

$$
S_{1}=\bigcup_{i=4}^{k} T_{i}=\left\{s_{2}, s_{3}, \ldots, s_{k+1}\right\} \quad \text { and } \quad S_{2}=\bigcup_{i=k+1}^{n} T_{i}=\left\{s_{k+1}, \ldots, s_{n}, s_{1}, s_{2}, s_{3}\right\} .
$$

Setting $m=k-3$, we define the cubical product map $m_{\square}$ to be

$$
\begin{aligned}
m_{\square}=f_{S_{1}} \times f_{S_{2}}: \mathfrak{M}_{0, S} & \longrightarrow \mathfrak{M}_{0, S_{1}} \times \mathfrak{M}_{0, S_{2}} \\
\left(x_{1}, \ldots, x_{\ell}\right) & \longmapsto\left(\left(x_{1}, \ldots, x_{m}\right),\left(x_{m+1}, \ldots, x_{\ell}\right)\right) .
\end{aligned}
$$

The cubical coordinates $f_{\square}$ defined above are obtained by iterating such maps.

Now, the simplicial case arises by considering the covering $S=\bigcup_{i=4}^{n} T_{i}$, where

$$
T_{i}=\left\{s_{1}, s_{2}, s_{3}, s_{i}\right\} \quad \text { for } \quad 4 \leq i \leq n .
$$

This defines a map

$$
f_{\triangle}: \mathfrak{M}_{0, S} \longrightarrow \prod_{i=4}^{n} \mathfrak{M}_{0, T_{i}}
$$

which satisfies condition (2.49) and is a non-degenerate coordinate system for the same reasons as above (namely $\left|T_{i} \cap T_{j}\right|=3$ for all $i \neq j$ ). We call $f_{\triangle}$ a system of simplicial coordinates on $\mathfrak{M}_{0, S}$. It is easy to check that $G_{f_{\triangle}}$ is in bijection with the symmetric group on $\ell$ letters

$$
G_{f_{\triangle}}=\mathfrak{S}\left(\left\{s_{4}, \ldots, s_{n}\right\}\right) \text {. }
$$

As above, we obtain an explicit set of simplicial coordinates by choosing the coordinate $t_{i}=[i+31 \mid 32]: \mathfrak{M}_{0, T_{i+3}} \cong \mathbb{P}^{1} \backslash\{0,1, \infty\}$, for $1 \leq i \leq \ell$. Thus (2.56) can be written

$$
f_{\triangle}=\left(t_{1}, \ldots, t_{\ell}\right): \mathfrak{M}_{0, S} \rightarrow\left(\mathbb{A}^{1} \backslash\{0,1\}\right)^{\ell},
$$

and we retrieve the isomorphism (2.3). As before, the domains $X_{T_{i}, \delta_{i}}$ map to unit intervals $(0,1)$ under $t_{i-3}$, and $X_{S, \delta}$ maps bijectively under $f_{\triangle}$ to the unit simplex $\left\{0<t_{1}<\cdots<t_{\ell}<1\right\}$. In this case, Equation (2.51) states that

$$
\coprod_{\sigma \in \mathfrak{S}\left(\left\{\sigma_{4}, \ldots, \sigma_{n}\right\}\right)} X_{S, \sigma}=f_{\triangle}^{-1}\left(\prod_{i} X_{T_{i}}\right)
$$


i.e., the unit cube $(0,1)^{\ell}$ is tesselated with $\ell$ ! copies of the unit simplex, up to boundary terms.

Now let $k \geq 4, m=k-3$, and set

$$
S_{1}=\bigcup_{i=4}^{k} T_{i}=\left\{s_{1}, s_{2}, s_{3}, \ldots, s_{k-1}, s_{k}\right\} \quad \text { and } \quad S_{2}=\bigcup_{i=k+1}^{n} T_{i}=\left\{s_{1}, s_{2}, s_{3}, s_{k+1}, \ldots, s_{n}\right\} .
$$

We define the simplicial product map $m_{\triangle}$ to be

$$
\begin{aligned}
m_{\triangle}=f_{S_{1}} \times f_{S_{2}}: \mathfrak{M}_{0, S} & \longrightarrow \mathfrak{M}_{0, S_{1}} \times \mathfrak{M}_{0, S_{2}} \\
\left(t_{1}, \ldots, t_{\ell}\right) & \longmapsto\left(\left(t_{1}, \ldots, t_{m}\right),\left(t_{m+1}, \ldots, t_{\ell}\right)\right) .
\end{aligned}
$$

In this case, the set $G_{m_{\triangle}}$ is exactly the set $\mathfrak{S}(m, \ell-m)$ of all possible ways of shuffling together the sets $\left\{s_{4}, \ldots, s_{k}\right\}$ and $\left\{s_{k+1}, \ldots, s_{n}\right\}$ whilst preserving the orderings $s_{4}<\cdots<s_{k}$ and $s_{k+1}<\cdots<s_{n}$. In explicit simplicial coordinates, which involves setting $s_{1}=1$, $s_{2}=\infty, s_{3}=0$, and $s_{i+3}=t_{i}$ for $1 \leq i \leq \ell$, Equation (2.51) is the well-known formula for the decomposition of a product of simplices:

$$
\begin{gathered}
\left\{0<t_{1}<\ldots<t_{m}<1\right\} \times\left\{0<t_{m+1}<\ldots<t_{\ell}<1\right\} \backslash\left\{t_{i}=t_{j}\right\} \cong \\
\coprod_{\sigma \in \mathfrak{S}(m, \ell-m)}\left\{0<t_{\sigma(1)}<\ldots<t_{\sigma(\ell)}<1\right\} .
\end{gathered}
$$

This plays an important role in the shuffle product for iterated integrals. The product maps defined above will be used to generalize such shuffle product formulae in $\S 7.5$.

\section{The reduced bar construction and Picard-Vessiot theory}

The main tool for computing the periods of moduli spaces is a triviality theorem for the cohomology of a variant of the bar construction on the de Rham complex of $\mathfrak{M}_{0, S}$. Although many of the results below hold in considerably greater generality, we consider the complement of an affine hyperplane arrangement $M$, which is more than adequate for our purposes. We first show that the reduced bar construction on $\Omega^{\star}(M)$ defines a Picard-Vessiot extension of its ring of regular functions. This is an abstract algebraic analogue of the ring of iterated integrals over $M$. Then, by showing that the bar construction decomposes as a tensor product over a fibration, we prove that the cohomology of the bar construction is trivial for fibre-type arrangements. This result is also proved for quadratic arrangements in the appendix. Our point of view, using differential Galois theory, is different from classical approaches to this subject $[9,10,31,32,39]$. The main technical idea is the notion of unipotent extensions of differentially simple algebras, which is developed in $\S 3.3$. The example $\mathfrak{M}_{0,5}$ is discussed in $\S 3.8$.

\subsection{Shuffle algebras and non-commutative formal power series}

Let $R$ be a commutative unitary ring. Let $k \geq 1$, let $A=\left\{a_{1}, \ldots, a_{k}\right\}$ denote an alphabet with $k$ symbols, and let $A^{*}$ denote the free non-commutative monoid generated by $A$, i.e., the 
set of all words $w$ in the symbols $a_{i}$, along with the empty word 1 . Let $R\langle A\rangle$ be the free noncommutative $R$-algebra generated by $A$. If $V_{1}$ is the free $R$-module with basis $\left\{a_{1}, \ldots, a_{k}\right\}$, and if we set $V_{m}=V_{1}^{\otimes m}$ and $V_{0}=R$, then clearly

$$
R\langle A\rangle=\bigoplus_{m \geq 0} V_{m}
$$

It is well-known that $R\langle A\rangle$ can be given the structure of a cocommutative graded Hopf algebra. The multiplication law on $R\langle A\rangle$ is given by concatenation of words, and the coproduct $\Gamma: R\langle A\rangle \rightarrow R\langle A\rangle \otimes R\langle A\rangle$ is defined to be the unique coproduct for which the elements of $A$ are all primitive:

$$
\Gamma\left(a_{i}\right)=a_{i} \otimes 1+1 \otimes a_{i} .
$$

The counit $\varepsilon: R\langle A\rangle \rightarrow R$ is given by projection onto the unit word 1. If $|w|$ denotes the number of symbols occuring in a word $w \in A^{*}$, then the antipode map is defined by $w \mapsto(-1)^{|w|} \widetilde{w}$, where the mirror map $w \mapsto \widetilde{w}$ reverses the order of the symbols in each word. One verifies that this defines a graded Hopf algebra structure.

Let $V_{1}^{\vee}$ denote the $R$-module dual to $V_{1}$, and let $A^{\prime}=\left\{a_{1}^{\prime}, \ldots, a_{k}^{\prime}\right\}$ denote the basis dual to $A$. Then $R\left\langle A^{\prime}\right\rangle$, the free tensor algebra over $V_{1}^{\vee}$, is the graded dual of $R\langle A\rangle$, and inherits a commutative Hopf algebra structure by duality. The multiplication law is now given by the shuffle product $\amalg: R\left\langle A^{\prime}\right\rangle \otimes R\left\langle A^{\prime}\right\rangle \rightarrow R\left\langle A^{\prime}\right\rangle$ which is defined recursively by the formulae: $w ш 1=1 ш w=w$, and

$$
a_{i}^{\prime} w_{1} ш a_{j}^{\prime} w_{2}=a_{i}^{\prime}\left(w_{1} ш a_{j}^{\prime} w_{2}\right)+a_{j}^{\prime}\left(a_{i}^{\prime} w_{1} ш w_{2}\right),
$$

for all words $w_{1}, w_{2} \in A^{\prime *}$, and all $a_{i}^{\prime}, a_{j}^{\prime} \in A^{\prime}$. This is a commutative, associative product with no zero divisors. The algebra $\left(R\left\langle A^{\prime}\right\rangle, \amalg\right)$ will be called the free shuffle algebra on the generators $a_{1}^{\prime}, \ldots, a_{k}^{\prime}$. The coproduct is defined by the map

$$
\begin{aligned}
\Delta: R\left\langle A^{\prime}\right\rangle & \rightarrow R\left\langle A^{\prime}\right\rangle \otimes R\left\langle A^{\prime}\right\rangle \\
\Delta(w) & =\sum_{u v=w} u \otimes v,
\end{aligned}
$$

and the antipode is given by the map $w \mapsto(-1)^{|w|} w$. The counit $\varepsilon: R\left\langle A^{\prime}\right\rangle \rightarrow R$ is given by projection onto the graded part of weight 0 , as previously.

Let $R\langle\langle A\rangle\rangle$ and $R\left\langle\left\langle A^{\prime}\right\rangle\right\rangle$ denote the completions of the graded algebras defined above with respect to the augmentation ideals ker $\varepsilon$. These are just the algebras of formal power series in $A, A^{\prime}$ respectively. The Hopf algebra structures $\Delta, \Gamma$, and $\varepsilon$ extend in the natural way to the completed algebras, and we shall denote them by the same symbols.

In addition, we introduce $k$ truncation operators $\partial_{a_{i}^{\prime}}$ for $1 \leq i \leq k$ :

$$
\begin{aligned}
\partial_{a_{i}^{\prime}}: R\left\langle A^{\prime}\right\rangle & \rightarrow R\left\langle A^{\prime}\right\rangle \\
\partial_{a_{i}^{\prime}}\left(a_{j}^{\prime} w\right) & =\delta_{i j} w,
\end{aligned}
$$

for all $a_{j}^{\prime} \in A^{\prime}, w \in A^{\prime *}$, where $\delta_{i j}$ is the Kronecker delta. It is easy to verify that the $\partial_{a_{i}^{\prime}}$ are derivations for the shuffle product, and furthermore, that this determines the shuffle product uniquely if we assume that 1 is the unit. The operators $\partial_{a_{i}^{\prime}}$ are dual to the operators $w \mapsto a_{i} w: R\langle A\rangle \rightarrow R\langle A\rangle$ which affix the letter $a_{i}$ to the left of words $w \in A^{*}$. That $\partial_{a_{i}^{\prime}}$ is a derivation is equivalent to the fact that $a_{i}$ is primitive for the coproduct $\Gamma$ by duality. 


\subsection{Arrangements of hyperplanes and the bar construction}

Consider an arrangement of $N$ hyperplanes $H_{1}, \ldots, H_{N}$ in affine space $\mathbb{A}^{\ell}$. Let $k$ denote a field of characteristic 0 over which the arrangement is defined. For each $1 \leq i \leq N$, choose a linear form $\alpha_{i} \in k\left[x_{1}, \ldots, x_{\ell}\right]$ such that $H_{i}$ is the divisor of zeros of $\alpha_{i}$. Let

$$
\mathcal{O}_{M}=k\left[x_{1}, \ldots, x_{\ell},\left\{\alpha_{i}^{-1}\right\}_{1 \leq i \leq N}\right]
$$

denote the ring of regular functions on the complement $M=\mathbb{A}_{k}^{\ell} \backslash \bigcup_{i} H_{i}$. We set

$$
d=\sum_{i=1}^{\ell} \frac{\partial}{\partial x_{i}} d x_{i} .
$$

Consider the de Rham complex of $\mathcal{O}_{M}$ :

$$
0 \longrightarrow \mathcal{O}_{M} \stackrel{d}{\longrightarrow} \Omega^{1}\left(\mathcal{O}_{M}\right) \stackrel{d}{\longrightarrow} \Omega^{2}\left(\mathcal{O}_{M}\right) \stackrel{d}{\longrightarrow} \ldots \stackrel{d}{\longrightarrow} \Omega^{\ell}\left(\mathcal{O}_{M}\right) \longrightarrow 0,
$$

where $\Omega^{r}\left(\mathcal{O}_{M}\right)=\bigoplus_{1 \leq i_{1}<\cdots<i_{r} \leq N} \mathcal{O}_{M} d x_{i_{1}} \wedge \cdots \wedge d x_{i_{r}}$ is placed in degree $r$. Let $H^{i}\left(\mathcal{O}_{M}\right)$, for $0 \leq i \leq \ell$, denote the corresponding cohomology groups. These are $k$-vector spaces. Since $M$ is affine, it follows that $H^{i}\left(\mathcal{O}_{M}\right)$ coincides with the de Rham hypercohomology of $M$ [30]. Consider the set of algebraic 1-forms:

$$
\omega_{i}=d \log \alpha_{i}, \quad \text { for } 1 \leq i \leq N .
$$

The following theorem is due to Arnold and Brieskorn [43, §5.4].

Theorem 3.1. - The cohomology ring $H^{\star}(M)$ is isomorphic to the graded k-algebra $A$ generated by the forms $\omega_{i}$, for $1 \leq i \leq N$.

In particular, the cohomology classes of the forms $\omega_{1}, \ldots, \omega_{N} \in \Omega^{1}\left(\mathcal{O}_{M}\right)$ are a $k$-basis for $H^{1}\left(\mathcal{O}_{M}\right)$. In this section, all tensor products will be taken over the field $k$ unless specified otherwise. Let $N$ denote the kernel of the exterior product

$$
N=\operatorname{ker}\left(\wedge: H^{1}\left(\mathcal{O}_{M}\right) \otimes H^{1}\left(\mathcal{O}_{M}\right) \longrightarrow H^{2}\left(\mathcal{O}_{M}\right)\right)
$$

We will not require the full strength of Theorem 3.1, only the following corollary.

Corollary 3.2. - If a form $\omega \in A$ is a coboundary $d \phi$, then it is zero.

It follows that $N$ is also the kernel of the map

$$
\wedge: \bigoplus_{1 \leq i, j \leq N} k \omega_{i} \otimes \omega_{j} \longrightarrow \Omega^{2}\left(\mathcal{O}_{M}\right) .
$$

For each positive integer $m \geq 2$, the vector space $V_{m}\left(\mathcal{O}_{M}\right)$ of integrable words in the forms $\omega_{i}$ of weight $m$ is defined to be

$$
V_{m}\left(\mathcal{O}_{M}\right)=\bigcap_{i+j=m-2} H^{1}\left(\mathcal{O}_{M}\right)^{\otimes i} \otimes N \otimes H^{1}\left(\mathcal{O}_{M}\right)^{\otimes j}
$$

This is just the intersection of the kernels of the maps $\wedge_{i}$ for $1 \leq i \leq m-1$ :

$$
\begin{aligned}
\wedge_{i}: H^{1}\left(\mathcal{O}_{M}\right)^{\otimes m} & \longrightarrow H^{1}\left(\mathcal{O}_{M}\right)^{\otimes i-1} \otimes H^{2}\left(\mathcal{O}_{M}\right) \otimes H^{1}\left(\mathcal{O}_{M}\right)^{\otimes m-i-1}, \\
\eta_{1} \otimes \cdots \otimes \eta_{m} & \longmapsto \eta_{1} \otimes \cdots \otimes\left(\eta_{i} \wedge \eta_{i+1}\right) \otimes \cdots \otimes \eta_{m} .
\end{aligned}
$$

$4^{\text {e }}$ SÉRIE - TOME $42-2009-\mathrm{N}^{\mathrm{o}} 3$ 
Its elements can be written as linear combinations of symbols

$$
\sum_{I=\left(i_{1}, \ldots, i_{m}\right)} c_{I}\left[\omega_{i_{1}}\left|\omega_{i_{2}}\right| \ldots \mid \omega_{i_{m}}\right]
$$

where $1 \leq i_{j} \leq N$, and $c_{I} \in k$, which satisfy the integrability condition:

$$
\sum_{I=\left(i_{1}, \ldots, i_{m}\right)} c_{I} \omega_{i_{1}} \otimes \cdots \otimes \omega_{i_{j-1}} \otimes\left(\omega_{i_{j}} \wedge \omega_{i_{j+1}}\right) \otimes \omega_{i_{j+2}} \otimes \cdots \otimes \omega_{i_{m}}=0
$$

for each $1 \leq j \leq m-1$. We set $V_{0}\left(\mathcal{O}_{M}\right)=k$, and $V_{1}\left(\mathcal{O}_{M}\right)=H^{1}\left(\mathcal{O}_{M}\right)=\bigoplus_{i=1}^{N} k \omega_{i}$, and define

$$
V\left(\mathcal{O}_{m}\right)=\bigoplus_{m \geq 0} V_{m}\left(\mathcal{O}_{M}\right)
$$

The vector space of homotopy-invariant iterated integrals is then defined to be

$$
B\left(\mathcal{O}_{M}\right)=\mathcal{O}_{M} \otimes V\left(\mathcal{O}_{M}\right),
$$

with the obvious grading. This is similar to the zero ${ }^{\text {th }}$ cohomology group of Chen's reduced bar complex on $\mathcal{O}_{M}$, which is usually written $H^{0}\left(B\left(\Omega^{\bullet} \mathcal{O}_{M}\right)\right)$, with the difference that it is made up of closed 1-forms only (see [32, 39, 10, 31]).

In order to define a differential on $B\left(\mathcal{O}_{M}\right)$, we let

$$
\Omega^{i} B\left(\mathcal{O}_{M}\right)=\Omega^{i}\left(\mathcal{O}_{M}\right) \otimes_{\mathcal{O}_{M}} B\left(\mathcal{O}_{M}\right)=\Omega^{i}\left(\mathcal{O}_{M}\right) \otimes_{k} V\left(\mathcal{O}_{M}\right),
$$

and define $d: \Omega^{i} B\left(\mathcal{O}_{M}\right) \rightarrow \Omega^{i+1} B\left(\mathcal{O}_{M}\right)$ by the formula

$$
\begin{aligned}
d \sum_{I=\left(i_{1}, \ldots, i_{m}\right)} \phi_{I} \otimes\left[\omega_{i_{1}}\left|\omega_{i_{2}}\right| \ldots \mid \omega_{i_{m}}\right] & =\sum_{I=\left(i_{1}, \ldots, i_{m}\right)}(-1)^{\operatorname{deg} \phi_{I}} \phi_{I} \wedge \omega_{i_{1}} \otimes\left[\omega_{i_{2}}|\ldots| \omega_{i_{m}}\right] \\
& +\sum_{I=\left(i_{1}, \ldots, i_{m}\right)} d \phi_{I} \otimes\left[\omega_{i_{1}}\left|\omega_{i_{2}}\right| \ldots \mid \omega_{i_{m}}\right],
\end{aligned}
$$

where $\phi_{I} \in \Omega^{i}\left(\mathcal{O}_{M}\right)$. It follows from the integrability condition (3.8) that $d^{2}=0$. We can therefore consider the following complex

$$
0 \longrightarrow B\left(\mathcal{O}_{M}\right) \stackrel{d}{\longrightarrow} \Omega^{1} B\left(\mathcal{O}_{M}\right) \stackrel{d}{\longrightarrow} \Omega^{2} B\left(\mathcal{O}_{M}\right) \stackrel{d}{\longrightarrow} \cdots \stackrel{d}{\longrightarrow} \Omega^{\ell} B\left(\mathcal{O}_{M}\right) \longrightarrow 0,
$$

where $\Omega^{i} B\left(\mathcal{O}_{M}\right)$ is placed in degree $i$. Its cohomology will be denoted $H_{\mathrm{DR}}^{i}\left(B\left(\mathcal{O}_{M}\right)\right)$. By Definition (3.9), $V\left(\mathcal{O}_{M}\right)$ is contained in the free $\mathcal{O}_{M}$ shuffle algebra generated by $V_{1}\left(\mathcal{O}_{M}\right)$, which is a commutative graded Hopf algebra:

$$
V\left(\mathcal{O}_{M}\right) \subset k\left\langle\omega_{1}, \ldots, \omega_{N}\right\rangle .
$$

The product $\amalg$ is the shuffle product defined in (3.1), and the coproduct $\Delta$ was defined in (3.2). One can verify that $V\left(\mathcal{O}_{M}\right)$ is preserved by $ш$ and $\Delta$, and is therefore a graded Hopf subalgebra of $\mathcal{O}_{M}\left\langle\omega_{1}, \ldots, \omega_{N}\right\rangle$.

COROLlary 3.3. - $B\left(\mathcal{O}_{M}\right)$ is a commutative graded algebra for the shuffle product $u$, and has a natural coproduct $\Delta: B\left(\mathcal{O}_{M}\right) \rightarrow B\left(\mathcal{O}_{M}\right) \otimes_{\mathcal{O}_{M}} B\left(\mathcal{O}_{M}\right)$. 


\subsection{Unipotent extensions of differentially simple algebras}

Let $k$ be a field of characteristic 0 , and let $R$ denote a commutative, unitary $k$-algebra with $\ell$ commuting derivations $\partial_{1}, \ldots, \partial_{\ell}$. Its de Rham complex begins as follows:

$$
0 \longrightarrow R \longrightarrow \bigoplus_{1 \leq i \leq \ell} R \longrightarrow \bigoplus_{1 \leq i<j \leq \ell} R \longrightarrow \ldots
$$

where the first map is given by $f \mapsto\left(\partial_{i} f\right)_{i}$, and the second map sends $\left(f_{1}, \ldots, f_{\ell}\right)$ to $\left(\partial_{i} f_{j}-\partial_{j} f_{i}\right)_{i<j}$. The ring of constants of $R$ is the $k$-algebra:

$$
H^{0}(R)=\bigcap_{i=1}^{\ell} \operatorname{ker} \partial_{i} .
$$

Definition 3.4. - We say that $R$ is differentially simple if $H^{0}(R)=k$, and if $R$ is a simple module over its ring of differential operators $R\left[\partial_{1}, \ldots, \partial_{\ell}\right]$.

Recall that a differential ideal of $R$ is an ideal $I \subset R$ such that $\partial_{i} I \subset I$ for all $1 \leq i \leq \ell$. It is immediate that $R$ is differentially simple if and only if it has no differential ideals apart from 0 and $R$. An equivalent condition is that for every non-zero $r \in R$, there exists an operator $D_{r} \in R\left[\partial_{1}, \ldots, \partial_{\ell}\right]$ such that $D_{r} r=1$. This is the analogue of the notion of a field in differential algebra.

Now let us assume that $R$ is differentially simple. Let $B$ be a differential $k$-algebra containing $R$, with differentials we also denote by $\partial_{1}, \ldots, \partial_{\ell}$.

Definition 3.5. - We say that $B$ is unipotent if $H^{0}(B)=k$, and if there exists a filtration by $R\left[\partial_{1}, \ldots, \partial_{\ell}\right]$-subalgebras $W^{i} B$ of $B$ :

$$
R=W^{0} B \subset W^{1} B \subset \cdots \subset W^{i+1} B \subset \cdots \subset B,
$$

such that $B=\bigcup W^{i} B$, and $W^{i+1} B$ is generated, as an algebra over $W^{i} B$, by finitely many elements $y$ such that $\partial_{1} y, \ldots, \partial_{\ell} y \in W^{i} B$.

In other words, $B$ is obtained by adding successive primitives to $R$ with respect to the operators $\partial_{1}, \ldots, \partial_{\ell}$. The following lemma is a variant of a well-known result concerning extensions of differential fields by adjoining primitives.

Lemma 3.6. - Let $R$ be a differentially simple $k$-algebra, and let $r_{1}, \ldots, r_{\ell} \in R$ such that $\partial_{i} r_{j}=\partial_{j} r_{i}$ for all $1 \leq i, j \leq \ell$. On the polynomial ring $R[y]$, we extend the derivations $\partial_{1}, \ldots, \partial_{\ell}$ by setting

$$
\partial_{i} y=r_{i} \in R \quad \text { for } 1 \leq i \leq \ell .
$$

The extended operators $\partial_{i}$ commute and are unique. Suppose that no element $u \in R$ satisfies $\partial_{i} u=r_{i}$ (i.e., the class of $\left(r_{1}, \ldots, r_{\ell}\right)$ is non-zero in $\left.H^{1}(R)\right)$. Then $R[y]$ is differentially simple.

Proof. - Let $I$ be a differential ideal in $R[y]$, and suppose that $f(y) \in I$ is a polynomial in $y$ of minimal degree $n \geq 1$ :

$$
f(y)=a_{n} y^{n}+a_{n-1} y^{n-1}+\cdots+a_{0} \in I,
$$

$4^{\text {e }}$ SÉRIE - TOME $42-2009-\mathrm{N}^{\mathrm{o}} 3$ 
where $a_{i} \in R, a_{n} \neq 0$. Since $R$ is differentially simple, there exists an operator $D \in R\left[\partial_{i}\right]$ such that $D a_{n}=1$. After applying this operator to the equation above, we may assume that $a_{n}=1$. On applying $\partial_{i}$, we obtain

$$
\left(n r_{i}+\partial_{i} a_{n-1}\right) y^{n-1}+\cdots+\left(a_{1} r_{i}+\partial_{i} a_{0}\right) \in I .
$$

By the minimality of $f(y)$, this polynomial is identically 0 , so the set of equations $\partial_{i} u=r_{i}$, for $1 \leq i \leq \ell$, already has a solution $u=-a_{n-1} / n \in R$. This contradicts the assumption, and proves that $R[y]$ has no non-trivial differential ideals.

REMARK 3.7. - Since $R[y]$ is differentially simple, it has no non-trivial quotients. For any differential $R$-algebra $R[\eta]$, where $\partial_{i} \eta \in R$ for $1 \leq i \leq \ell$, and $\eta$ satisfies the conditions of the lemma, the element $\eta$ is therefore transcendental.

COROLlary 3.8. - Let $B$ denote a unipotent extension of $R$, where $R$ is differentially simple. Then $B$ is a polynomial algebra, and every differential $R$-subalgebra of $B$ is differentially simple.

Proof. - Let $A$ denote a differential $R$-subalgebra of $B$. We can formally add primitives $y_{1}, \ldots, y_{p}, \ldots$ to $R$, where $y_{p} \in A$, to obtain a sequence of differential algebras

$$
R \subset R\left[y_{1}\right] \subset R\left[y_{1}, y_{2}\right] \subset \cdots \subset A=R\left[y_{1}, \ldots, y_{p}, \ldots\right] .
$$

We can assume that each inclusion is strict, i.e., $y_{p+1}$ is not in $R\left[y_{1}, \ldots, y_{p}\right]$ for each $p \geq 0$. Let

$$
\partial_{i} y_{p+1}=r_{p+1, i} \in R\left[y_{1}, \ldots, y_{p}\right] .
$$

Since the ring of constants of $B$ is $k$, it follows that the primitive $y_{p+1}$ is the unique solution to the equations $\partial_{i} u=r_{p+1, i}$ for $1 \leq i \leq \ell$ in $B$, up to some constant in $k$. Applying the previous lemma inductively, we deduce that $R\left[y_{1}, \ldots, y_{p}\right]$ is differentially simple and pure transcendent for all $p \geq 1$. It follows that $A$ is differentially simple, and that $A$ is a polynomial algebra.

Definition 3.9. - Let $B$ denote a unipotent extension of a differentially simple $k$-algebra $R$. We say that $B$ is a unipotent closure of $R$ if

$$
H^{0}(B)=k, \quad \text { and } \quad H^{1}(B)=0 .
$$

A unipotent closure is closed under the operation of taking 1-primitives: for all $f_{1}, \ldots, f_{\ell} \in B$ such that $\partial_{i} f_{j}=\partial_{j} f_{i}$ for all $1 \leq i, j \leq \ell$, there exists a primitive $F \in B$ such that $\partial_{1} F=f_{1}, \ldots, \partial_{\ell} F=f_{\ell}$.

Definition 3.10. - A pointed differential $k$-algebra $(R, \varepsilon)$ is a differential $k$-algebra $R$ and a $k$-linear homomorphism of algebras $\varepsilon: R \rightarrow k$. Now suppose that $R$ is differentially simple. We define $\mathfrak{u p}(R, \varepsilon)$ to be the category of unipotent pointed extensions of $(R, \varepsilon)$. Its objects are $\left(B, \varepsilon^{\prime}\right)$, where $B$ is a unipotent extension of $R$, such that the composition 
$R \rightarrow B \stackrel{\varepsilon^{\prime}}{\rightarrow} k$ coincides with $\varepsilon: R \rightarrow k$. A morphism $\phi$ from $\left(B_{1}, \varepsilon_{1}\right)$ to $\left(B_{2}, \varepsilon_{2}\right)$, is given by a commutative diagram:

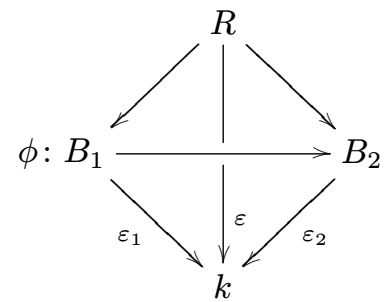

Any object $B \in \mathfrak{u p}(R, \varepsilon)$ is differentially simple by the previous corollary. It follows that morphisms in $\mathfrak{u p}(R, \varepsilon)$ are necessarily injective.

Lemma 3.11. - Morphisms in $\mathfrak{u p}(R, \varepsilon)$ are unique.

Proof. - Consider two morphisms $\phi, \phi^{\prime}:\left(B_{1}, \varepsilon_{1}\right) \rightarrow\left(B_{2}, \varepsilon_{2}\right)$ of pointed unipotent algebras over $(R, \varepsilon)$. If $\partial_{i}$, for $1 \leq i \leq \ell$, are the differentials on $R$, we denote their extensions to $B_{1}$ and $B_{2}$ by the same symbols. Let $W^{\bullet} B_{1}$ denote a filtration on $B_{1}$ as in Definition 3.5, and suppose by induction that $\phi=\phi^{\prime}$ on $W^{p} B_{1}$. Let $y \in W^{p+1} B$ such that $\partial_{i} y \in W^{p} B_{1}$ for all $1 \leq i \leq \ell$. Then

$$
\partial_{i}\left(\phi-\phi^{\prime}\right)(y)=\left(\phi-\phi^{\prime}\right)\left(\partial_{i} y\right)=0, \quad \text { for all } 1 \leq i \leq \ell,
$$

and therefore $\phi(y)-\phi^{\prime}(y) \in H^{0}\left(B_{2}\right)=k$. Since $\varepsilon_{2} \phi(y)=\varepsilon_{2} \phi^{\prime}(y)=\varepsilon_{1} y$, it follows that $\phi(y)=\phi^{\prime}(y)$. Thus $\phi=\phi^{\prime}$ on $W^{p+1} B$ and the uniqueness follows by induction.

Proposition 3.12. - Let $\left(R,\left\{\partial_{i}\right\}_{1 \leq i \leq \ell}, \varepsilon\right)$ and $\left(R^{\prime},\left\{\partial_{i}^{\prime}\right\}_{1 \leq i \leq \ell}, \varepsilon^{\prime}\right)$ denote two differentially simple pointed $k$-algebras, and let $\phi:(R, \varepsilon) \longrightarrow\left(R^{\prime}, \varepsilon^{\prime}\right)$ be a non-zero differential homomorphism. Let $\left(U, \varepsilon^{\prime}\right)$ be a unipotent closure of $\left(R^{\prime}, \varepsilon^{\prime}\right)$, and let $(B, \varepsilon)$ be any unipotent extension of $(R, \varepsilon)$. Then there is a unique morphism of differential algebras $\phi_{*}: B \longrightarrow U$ which extends $\phi$ and which is necessarily injective, such that the following diagram commutes:

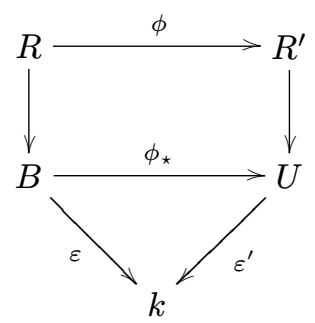

The map $\phi_{*}$ preserves any given filtrations on $B$ and $U$, i.e., $\phi\left(W^{p} B\right) \subset W^{p} U$ for all $p \geq 0$. If, furthermore, $H^{1}(B)=0$ and $\phi$ is an isomorphism, then $\phi_{*}$ is also an isomorphism.

Proof. - Suppose by induction that $\phi_{*}$ has been defined on $W^{p} B$. Since $B$ is unipotent, $W^{p+1} B$ is generated by elements $y$ such that $\partial_{i} y \subset W^{p} B$. For such $y$,

$$
\partial_{j}^{\prime} \phi_{*}\left(\partial_{i} y\right)=\phi_{*}\left(\partial_{j} \partial_{i} y\right)=\phi_{*}\left(\partial_{i} \partial_{j} y\right)=\partial_{i}^{\prime} \phi_{*}\left(\partial_{j} y\right) .
$$

Since $H^{1}(U)=0$, there exists $f \in U$ such that $\partial_{i}^{\prime} f=\phi_{*}\left(\partial_{i} y\right)$ for all $1 \leq i \leq \ell$. We extend the definition of $\phi_{*}$ by setting $\phi_{*}(y)=f+k_{y}$, where the constant of integration $k_{y} \in k$ is chosen 
such that $k_{y}+\varepsilon^{\prime}(f)=\varepsilon(y)$. By the previous lemma, $y$ is transcendent, and therefore $\phi_{*}$ is well-defined. We obtain a map $\phi_{*}$ on the whole of $B$ by induction. The previous corollary implies that $B$ is differentially simple. It follows that $\phi_{*}$ is injective because its kernel is a differential ideal in $B$ not equal to $B$ itself. The fact that $\phi_{*}$ preserves the filtrations is clear from the construction.

Now suppose that $\phi$ is an isomorphism and that $H^{1}(B)=0$. Applying the same construction to $\phi^{-1}$, we obtain a map $\left(\phi^{-1}\right)_{*}: U \rightarrow B$. Because of the uniqueness of morphisms in $\mathfrak{u p}(R, \varepsilon), \phi_{*}\left(\phi^{-1}\right)_{*}$ is the identity, and therefore $\phi_{*}$ is an isomorphism.

Corollary 3.13. - A pointed unipotent closure $(U, \varepsilon)$ over $(R, \varepsilon)$ is a final object in $\mathfrak{u p}(R, \varepsilon)$, i.e., every unipotent extension $(U, \varepsilon) \rightarrow\left(B, \varepsilon^{\prime}\right)$ is an isomorphism.

We can therefore speak of the unipotent closure $U$ of a pointed differentially simple ring $(R, \varepsilon)$ whenever it exists. Since $U$ is a union of polynomial algebras (Corollary 3.8), it necessarily has a $k$-valued point over $\varepsilon$.

Definition 3.14. - If $U$ is the unipotent closure of a differentially simple $k$-algebra $R$, let $\operatorname{Gal}(U / R)$ be the group of differential automorphisms $\phi: U \rightarrow U$ over $R$.

It follows from the definitions that $\operatorname{Gal}(U / R)$ is a pro-unipotent group. Now let $\epsilon: R \rightarrow k$ denote a $k$-valued point on $\operatorname{Spec} R$. The set of $k$-valued points $\left\{\phi \in \operatorname{Hom}_{k}(U, k):\left.\phi\right|_{R}=\varepsilon\right\}$ on $\operatorname{Spec} U$ lying above $\varepsilon$, is a principal homogeneous space over $\operatorname{Gal}(U / R)$. There is thus a complete analogy between the theory of unipotent differentially simple extensions and the theory of covering spaces.

\subsection{Base points at infinity}

We need to repeat the theory of unipotent closures in the case where the base points are at infinity. In order to do this, we need to generalise the notion of a $k$-valued point for certain differential algebras.

Definition 3.15. - Let $k$ be a field. We define

$$
k\left\{\epsilon_{1}, \ldots, \epsilon_{\ell}\right\}=k\left[\left[\epsilon_{1}, \ldots, \epsilon_{\ell}\right]\right]\left[\frac{1}{\epsilon_{1}}, \ldots, \frac{1}{\epsilon_{\ell}}\right],
$$

to be the differential $k$-algebra of Laurent series in $\epsilon_{i}$, equipped with $\ell$ commuting differentials $\partial_{\epsilon_{i}}$, for $1 \leq i \leq \ell$. Now define the extension

$$
U\left\{\epsilon_{1}, \ldots, \epsilon_{\ell}\right\}=k\left\{\epsilon_{1}, \ldots, \epsilon_{\ell}\right\}\left[L_{\epsilon_{1}}, \ldots, L_{\epsilon_{\ell}}\right],
$$

where $L_{\epsilon_{i}}$ is the formal logarithm of $\epsilon_{i}$, i.e., $\partial_{\epsilon_{i}} L_{\epsilon_{i}}=\epsilon_{i}^{-1}$ for $1 \leq i \leq \ell$.

The ring of constants of $k\left\{\epsilon_{1}, \ldots, \epsilon_{\ell}\right\}$ is $k$, and the extension $U\left\{\epsilon_{1}, \ldots, \epsilon_{\ell}\right\}$ is easily verified to be a unipotent closure of $k\left\{\epsilon_{1}, \ldots, \epsilon_{\ell}\right\}$, since $H^{0}\left(U\left\{\epsilon_{1}, \ldots, \epsilon_{\ell}\right\}\right)=k$, and $H^{1}\left(U\left\{\epsilon_{1}, \ldots, \epsilon_{\ell}\right\}\right)=0$. 
Definition 3.16. - Let $R$ be a differentially simple $k$-algebra with $\ell$ commuting differentials $\partial_{1}, \ldots, \partial_{\ell}$. We define a $k\left\{\epsilon_{1}, \ldots, \epsilon_{\ell}\right\}$-point on $R$ to be a $k$-linear homomorphism

$$
p: R \longrightarrow k\left\{\epsilon_{1}, \ldots, \epsilon_{\ell}\right\}
$$

which satisfies

$$
p \partial_{i}=\partial_{\epsilon_{i}} p, \quad \text { for all } \quad 1 \leq i \leq \ell .
$$

A $k\left\{\epsilon_{1}, \ldots, \epsilon_{\ell}\right\}$-point $p: R \rightarrow k\left\{\epsilon_{1}, \ldots, \epsilon_{\ell}\right\}$ defines an ordinary $k$-valued point if it factorises through $R \rightarrow k\left[\left[\epsilon_{1}, \ldots, \epsilon_{\ell}\right]\right]$ :

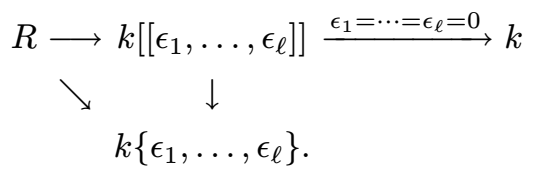

At the other extreme, we say that the point $p$ is at infinity if $\epsilon_{1}^{-1}, \ldots, \epsilon_{\ell}^{-1} \in \operatorname{Im} p$.

Example 3.17. - Consider the case where $k=\mathbb{Q}$, and $R=\mathbb{Q}[x, 1 / x, 1 /(1-x)]$ with differential $\partial / \partial x$. This corresponds to the projective line minus three points $\mathbb{P}^{1} \backslash\{0,1, \infty\}=$ $\mathbb{A}^{1} \backslash\{0,1\}$. The set of $k$-valued points on $R$ is the set $k \backslash\{0,1\}$. Each $k\{\epsilon\}$-point

$$
p: \mathbb{Q}\left[x, \frac{1}{x}, \frac{1}{1-x}\right] \longrightarrow k\{\epsilon\}
$$

satisfies $\partial_{\epsilon} p(x)=p\left(\partial_{x} x\right)=1$, and takes $x$ to $\epsilon+c$, where $c \in k$. The set of $k\{\epsilon\}$-points is therefore the set $k$. In this case, there are just two points at infinity, given by the maps $p_{\lambda}: R \rightarrow k\{\epsilon\}$, where $\lambda=0,1$; defined as follows:

$$
\begin{aligned}
x & \mapsto \epsilon+\lambda \\
\frac{1}{x-\lambda} & \mapsto \frac{1}{\epsilon} .
\end{aligned}
$$

More generally, every corner of the Stasheff polytope $\bar{X}_{S, \delta} \subset \mathfrak{M}_{0, S}^{\delta}$ defines a base-point at infinity on the ring $\mathcal{O}\left(\mathfrak{M}_{0, S}\right)$. Given a triangulation $\alpha \in \chi_{S, \delta}^{\ell}$ of the $n$-gon $(S, \delta)$, a set of vertex coordinates $x_{1}^{\alpha}, \ldots, x_{\ell}^{\alpha}(\S 2.6)$ gives rise to a map

$$
\left(\mathcal{O}\left(\mathfrak{M}_{0, S}\right), \partial / \partial x_{i}^{\alpha}\right) \longrightarrow k\left\{\epsilon_{1}, \ldots, \epsilon_{\ell}\right\}
$$

which sends $x_{i}^{\alpha}$ to $\epsilon_{i}$ for $1 \leq i \leq \ell$.

A point at infinity corresponds to a point which is the intersection of a number of normal crossing divisors, and will play the role of a tangential base point.

Definition 3.18. - Let $R$ denote any differentially simple $k$-algebra, with derivations $\partial_{1}, \ldots, \partial_{\ell}$. We define a logarithmic Laurent expansion to be a homomorphism of differential $k$-algebras:

$$
\phi: R \longrightarrow U\left\{\epsilon_{1}, \ldots, \epsilon_{\ell}\right\}
$$

There is a natural map $\lambda: U\left\{\epsilon_{1}, \ldots, \epsilon_{\ell}\right\} \longrightarrow k$ which projects on to the constant coefficient in the logarithmic Laurent series. It factorises through

$$
U\left\{\epsilon_{1}, \ldots, \epsilon_{\ell}\right\} \longrightarrow k\left\{\epsilon_{1}, \ldots, \epsilon_{\ell}\right\} \longrightarrow k,
$$


where the first map sends $L_{\epsilon_{i}}$ to 0 for $1 \leq i \leq \ell$, and the second map picks out the constant term in the Laurent expansion

$$
\sum_{i_{1}, \ldots, i_{\ell} \geq-N} a_{i_{1}, \ldots, i_{\ell}} \epsilon_{1}^{i_{1}} \ldots \epsilon_{\ell}^{i_{\ell}} \quad \mapsto \quad a_{0, \ldots, 0}
$$

The map $\lambda$ has a certain number of formal properties, which we will not require explicitly. Given a logarithmic Laurent expansion $\phi: R \rightarrow U\left\{\epsilon_{1}, \ldots, \epsilon_{\ell}\right\}$, we define the map of constants of $\phi$ to be the ( $k$-linear, additive) map

$$
\lambda \circ \phi: R \rightarrow k .
$$

Lemma 3.19. - Let $R$ be a differentially simple $k$-algebra, and let $p: R \rightarrow k\left\{\epsilon_{1}, \ldots, \epsilon_{\ell}\right\}$ denote a $k\left\{\epsilon_{1}, \ldots, \epsilon_{\ell}\right\}$-point. Let $B$ denote a unipotent extension of $R$. Consider any logarithmic Laurent expansion $\phi: B \rightarrow U\left\{\epsilon_{1}, \ldots, \epsilon_{\ell}\right\}$ over the point $p$, i.e., such that the following diagram commutes:

$$
\begin{gathered}
B \stackrel{\phi}{\longrightarrow} U\left\{\epsilon_{1}, \ldots, \epsilon_{\ell}\right\} \\
\uparrow \\
R \stackrel{p}{\longrightarrow} k\left\{\epsilon_{1}, \ldots, \epsilon_{\ell}\right\} .
\end{gathered}
$$

Then $\phi$ is uniquely determined by its map of constants $\lambda \circ \phi: B \rightarrow k$.

Proof. - This follows immediately using the method of proof of Proposition 3.12.

A map of constants amounts to choosing a constant of integration for each successive primitive in a unipotent extension $B$ of $R$. We can now copy the results of the previous sections for base points at infinity.

Definition 3.20. - Let $R$ denote a differentially simple $k$-algebra, and let $p: R \rightarrow k\left\{\epsilon_{1}, \ldots, \epsilon_{\ell}\right\}$ be a $k\left\{\epsilon_{1}, \ldots, \epsilon_{\ell}\right\}$-point. Let $\mathfrak{u t}(R, p)$ denote the category of pointed unipotent extensions of $(R, p)$, whose objects are unipotent $R$-algebras $(B, \phi)$, where $\phi: B \rightarrow U\left\{\epsilon_{1}, \ldots, \epsilon_{\ell}\right\}$ is a logarithmic Laurent expansion (or, equivalently, the corresponding map of constants). Morphisms are defined in a similar manner to the category $\mathfrak{u p}$.

The proof of Lemma 3.11 and Proposition 3.12 go through without any difficulty.

Proposition 3.21. - Morphisms are unique in $\mathfrak{u t}(R, p)$, and a unipotent closure $U$ of $R$ is a final object in the category $\mathfrak{u t}(R, p)$.

If $U$ is the unipotent closure of $(R, p)$, where $p$ is a $k\left\{\epsilon_{1}, \ldots, \epsilon_{\ell}\right\}$-point, then

$$
\left\{\phi: U \rightarrow U\left\{\epsilon_{1}, \ldots, \epsilon_{\ell}\right\},\left.\phi\right|_{R}=p\right\}
$$

is a principal homogeneous space over $\operatorname{Gal}(U / R)$. 


\subsection{One-dimensional fibrations and their relative unipotent closures}

Let $R$ denote a differentially simple $k$-algebra, with commuting differentials $\partial_{1}, \ldots, \partial_{\ell}$. Suppose that we are given $N$ elements $f_{1}, \ldots, f_{N} \in R$ which satisfy the condition

$$
\frac{1}{f_{i}-f_{j}} \in R \quad \text { for all } 1 \leq i<j \leq N .
$$

Consider the $R$-algebra

$$
\widehat{R}=R\left[y, \frac{1}{y-f_{1}}, \ldots, \frac{1}{y-f_{N}}\right]
$$

equipped with the derivation $\partial_{y}$ which is the unique $R$-linear derivation satisfying $\partial_{y} y=1$. Clearly $\partial_{i}, \partial_{y}$ commute for all $1 \leq i \leq \ell$. Consider the free shuffle algebra generated by the symbols $\omega_{1}, \ldots, \omega_{N}$ over $\widehat{R}$ :

$$
U_{\widehat{R} / R}=\widehat{R} \otimes_{k} k\left\langle\omega_{1}, \ldots, \omega_{N}\right\rangle,
$$

and let us extend the definition of $\partial_{y}$ to $U_{\widehat{R} / R}$ by setting

$$
\partial_{y}=\partial_{y} \otimes 1+\sum_{i=1}^{N} \frac{1}{y-f_{i}} \otimes \partial_{\omega_{i}},
$$

where the left truncation operators $\partial_{\omega_{i}}$ were defined in $\S 3.1$. This makes $U_{\widehat{R} / R}$ into a differential $R$-algebra. A similar algebra was considered in [41], and a specific case is studied in detail in $\S 5$. The symbol $\omega_{i}$ represents the formal $\operatorname{logarithm} \log \left(y-f_{i}\right)$, for $1 \leq i \leq N$. By analogy with the bar construction, we will write $\left[\omega_{i_{1}}|\ldots| \omega_{i_{m}}\right]$ for the tensor $\omega_{i_{1}} \otimes \cdots \otimes \omega_{i_{m}}$. The following proposition states that $U_{\widehat{R} / R}$ is a relative unipotent closure over the base $R$.

Proposition 3.22. $-H^{0}\left(U_{\widehat{R} / R}\right)=R$ and $H^{1}\left(U_{\widehat{R} / R}\right)=0$.

Proof. - It is a simple exercise to show that the ring of constants of $U_{\widehat{R} / R}$ is $R$. The argument is given in the proof of Lemma 3.31, and works in complete generality. The fact that $H^{1}\left(U_{\widehat{R} / R}\right)$ vanishes is equivalent to the existence of primitives with respect to $\partial_{y}$ over the base $R$. The key observation is the following identity, which is valid in $U_{\widehat{R} / R}$, by assumption (3.16):

$$
\frac{1}{\left(y-f_{i}\right)\left(y-f_{j}\right)}=\frac{1}{f_{i}-f_{j}}\left(\frac{1}{y-f_{i}}-\frac{1}{y-f_{j}}\right) \text {. }
$$

Using this, we can decompose elements of $\widehat{R}$ into partial fractions. It suffices, therefore, to find primitives of expressions of the form

$$
\frac{1}{\left(y-f_{i}\right)^{n}}\left[\omega_{i_{1}}|\ldots| \omega_{i_{m}}\right]
$$

where $n \in \mathbb{Z}$, and $1 \leq i_{1}, \ldots, i_{m} \leq N$. If $n=-1$, a primitive is given by

$$
\left[\omega_{i}\left|\omega_{i_{1}}\right| \ldots \mid \omega_{i_{m}}\right]
$$

by definition. For other values of $n$, we can reduce to this case by integrating by parts and using induction. It follows that every element in $U_{\widehat{R} / R}$ has a primitive with respect to $\partial_{y}$. 
Note that there is no integrability condition to be verified because the fibres of the map Spec $\widehat{R} \rightarrow$ Spec $R$ are one-dimensional. We now show how to differentiate the symbols $\left[\omega_{i_{1}}|\ldots| \omega_{i_{m}}\right]$ with respect to the operators $\partial_{1}, \ldots, \partial_{\ell}$ of the base ring $R$ (differentiation under an iterated integral). To do this, consider an $R$-linear map

$$
p: \widehat{R} \longrightarrow R\{\epsilon\}=R[[\epsilon]]\left[\frac{1}{\epsilon}\right]
$$

which satisfies $p \partial_{y}=\partial_{\epsilon} p$. Then there is a unique logarithmic Laurent expansion:

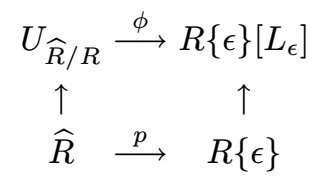

such that the map of constants is zero on the generators of $U_{\widehat{R} / R}$, i.e.,

$$
\begin{aligned}
\lambda \circ \phi: U_{\widehat{R} / R} & \longrightarrow R \\
{\left[\omega_{i_{1}}|\ldots| \omega_{i_{m}}\right] } & \longmapsto 0 .
\end{aligned}
$$

This follows from the inductive method of proof of Proposition 3.12: if $w=\left[\omega_{i_{1}}|\ldots| \omega_{i_{m}}\right]$, and $\phi\left(\partial_{y} w\right)=a$ has already been defined, then $\phi(w)$ is defined to be a primitive of $a$ with respect to $\partial_{\epsilon}$. The constant of integration is normalised by the condition $\lambda(\phi(w))=0$, since the map (3.21) is $R$-linear.

Proposition 3.23. - The action of the differential operators $\partial_{i}$ on $R$, for $1 \leq i \leq \ell$, extend uniquely to $U_{\widehat{R} / R}$ in such a way that the $\partial_{i}$ commute with each other, and such that:

$$
\left[\partial_{i}, \phi\right]=\left[\partial_{i}, \partial_{y}\right]=0 \quad \text { for all } 1 \leq i \leq \ell .
$$

For each element $w=\left[\omega_{i_{1}}|\ldots| \omega_{i_{m}}\right] \in W^{m} U_{\widehat{R} / R}, \partial_{i} w \in W^{m-1} U_{\widehat{R} / R}$. It follows that $U_{\widehat{R} / R}$ is a unipotent differential algebra with respect to all the operators $\partial_{1}, \ldots, \partial_{\ell}, \partial_{y}$.

Proof. - The map $\phi$ is injective, since $U_{\widehat{R} / R}$ is differentially simple, and therefore the action of the operators $\partial_{i}$ on $U_{\widehat{R} / R}$ are induced from $R\{\epsilon\}\left[L_{\epsilon}\right]$ by restriction. More precisely, suppose by induction that the action of the operators $\partial_{i}$ have already been defined on $W^{p} U_{\widehat{R} / R}$, for some $p \geq 0$. Let $w \in W^{p+1} U_{\widehat{R} / R}$ such that $\partial_{y} w \in W^{p} U_{\widehat{R} / R}$. If we view $U_{\widehat{R} / R}$ as a subalgebra of $R\{\epsilon\}\left[L_{\epsilon}\right]$, then we can write

$$
\partial_{y} \partial_{i} w=\partial_{i} \partial_{y} w \in U_{\widehat{R} / R}
$$

The element $\partial_{i} w$, which is a priori in $R\{\epsilon\}\left[L_{\epsilon}\right]$, in fact lies in $U_{\widehat{R} / R}$. This is because it is a primitive of $\partial_{i} \partial_{y} w \in U_{\widehat{R} / R}$, and we know that $H^{1}\left(U_{\widehat{R} / R}\right)=0$, and $H^{0}\left(R\{\epsilon\}\left[L_{\epsilon}\right]\right)=R$. More explicitly, if $w=\left[\omega_{i_{1}}|\ldots| \omega_{i_{m}}\right]$, then we define

$$
\partial_{i} w=(1-\lambda \circ \phi) A,
$$

where $A$ is any solution in $U_{\widehat{R} / R}$ to $\partial_{y} A=\partial_{i} \partial_{y} w$. The fact that the operators $\partial_{i}$ decrease the weight of each such element $w$ is easily proved by induction and is left to the reader. 
For each $1 \leq i \leq N$, there is a unique $R$-linear map

$$
\begin{aligned}
p: \widehat{R} & \rightarrow R\{\epsilon\} \\
y-f_{i} & \mapsto \epsilon,
\end{aligned}
$$

such that $\partial_{\epsilon} p=p \partial_{y}$. It satisfies:

$$
p\left(\frac{1}{y-f_{j}}\right)=\frac{1}{f_{i}-f_{j}} \sum_{k \geq 0} \frac{\epsilon^{k}}{\left(f_{j}-f_{i}\right)^{k}} \quad \text { for all } j \neq i .
$$

Corollary 3.24. - Suppose that $U_{R}$ is the unipotent closure of $R$. Then the algebra $U_{R} \otimes_{R} U_{\widehat{R} / R}$ is the unipotent closure of $\widehat{R}$.

Proof. - By choosing any map $p$ given by Equation (3.22) above, we obtain a differential $\widehat{R}\left[\partial_{1}, \ldots, \partial_{\ell}, \partial_{y}\right]$-structure on $U_{R} \otimes_{R} U_{\widehat{R} / R}$. It is clear that a tensor product of unipotent algebras is unipotent, and that $H^{0}\left(U_{R} \otimes_{R} U_{\widehat{R} / R}\right)=k$. Since the operator $\partial_{y}$ is zero on $U_{R}$, it follows that $H^{1}\left(U_{R} \otimes_{R} U_{\widehat{R} / R}\right)=0$. Concretely, in order to find 1-primitives in this algebra, first take a primitive with respect to $\partial_{y}$ and then adjust the constant of integration in $U_{R}$ using the fact that $H^{1}\left(U_{R}\right)=0$.

By iterating the previous corollary, we deduce that any differentially simple algebra $R$ which is of fibre-type (i.e., an iterated sequence of fibrations) has an explicit unipotent closure which is a tensor product of shuffle algebras.

TheOREM 3.25. - Let $R$ denote a differentially simple $k$-algebra, which can be expressed as a finite series of extensions of the type (3.17) satisfying (3.16):

$$
k=R_{0} \subset R_{1} \subset \cdots \subset R_{n}=R,
$$

where

$$
R_{t}=R_{t-1}\left[y_{t},\left(\frac{1}{y_{t}-f_{t, i}}\right)_{1 \leq i \leq N_{t}}\right],
$$

and $f_{t, i}-f_{t, j}$ is invertible in $R_{t-1}$ for all $1 \leq i<j \leq N_{t}$, and all $t=1, \ldots, n$. Then the unipotent closure $U_{R}$ of $(R, p)$ exists, and is isomorphic (as an algebra) to the tensor product of free shuffle algebras on $N_{t}$ generators, for $1 \leq t \leq n$ :

$$
U_{R} \cong R \otimes_{k} \bigotimes_{t=1}^{n} k\left\langle\omega_{t, 1}, \ldots, \omega_{t, N_{t}}\right\rangle .
$$

Its differential structure is uniquely determined by such a tensor decomposition.

Proof. - This follows immediately from the previous corollary by induction. The differential structure is determined by the construction in Proposition 3.23.

We therefore have an explicit description of the algebraic structure of the unipotent closure of $R$ for any $R$ which is of fibre type. Note that there may be several natural isomorphisms of the form (3.25), even after fixing base-points.

$4{ }^{\mathrm{e}}$ SÉRIE - TOME $42-2009-\mathrm{N}^{\mathrm{o}} 3$ 


\subsection{Iterated integrals}

Let $\mathcal{O}_{M}$ denote the ring of regular functions on an affine hyperplane arrangement as considered in $\S 3.2 . \mathcal{O}_{M}$ is a differential algebra with $\ell$ commuting differentials $\partial / \partial x_{1}, \ldots, \partial / \partial x_{\ell}$. Suppose that $I \subset \mathcal{O}_{M}$ is any non-zero differential ideal. It must contain a polynomial $P \in k\left[x_{1}, \ldots, x_{\ell}\right]$, since we can multiply by suitable powers of the hyperplane equations $\alpha_{i}$ to clear denominators. It is clear that there exists a polynomial $D_{P}$ in the $\partial / \partial x_{i}$ such that $D_{P} P=1$, and therefore $I=\mathcal{O}_{M}$. It follows that $\mathcal{O}_{M}$ is differentially simple.

Theorem 3.26. - The de Rham cohomology of $B\left(\mathcal{O}_{M}\right)$ satisfies:

$$
H_{\mathrm{DR}}^{0}\left(B\left(\mathcal{O}_{M}\right)\right)=k, \quad \text { and } H_{\mathrm{DR}}^{1}\left(B\left(\mathcal{O}_{M}\right)\right)=0,
$$

and $B\left(\mathcal{O}_{M}\right)$ is the unipotent closure of $\mathcal{O}_{M}$. It follows that every differential $\mathcal{O}_{M}$-subalgebra of $B\left(\mathcal{O}_{M}\right)$ is differentially simple, and $B\left(\mathcal{O}_{M}\right)$ is a polynomial algebra.

The proof of this theorem is postponed until $\S 3.7$.

REMARK 3.27. - The theorem in fact holds in much greater generality. Let $F$ denote any differential algebra such that $H^{1}(F) \cong \bigoplus_{i=1}^{N} k \omega_{i}$, where $\omega_{i} \in \Omega^{1}(F)$ satisfy

$$
\left(\bigoplus_{i, j} k \omega_{i} \wedge \omega_{j}\right) \cap d \Omega^{1}(F)=0 .
$$

If $k$ is the field of constants of $F$, and if $B(F)$ is defined as in $\S 3.2$, then it is clear from the proof $(\S 3.7)$, that $H^{0}(B(F))=k$ and $H^{1}(B(F))=0$. Furthermore, when $F$ is differentially simple, every differential $F$-subalgebra of $B(F)$ is differentially simple, and $B(F)$ is a polynomial algebra.

We now recall the definition of Chen's iterated integrals, which will give an isomorphism of the abstract algebra $B(F)$ with an algebra of multi-valued functions. Let $\widehat{M}$ be a universal covering for $M$, and let $p: \widehat{M} \rightarrow M$ denote the projection map. Let $b \in M$ denote a base point for $M$. Given any smooth path $\gamma:[0,1] \rightarrow M$ beginning at $b$, and holomorphic 1-forms $\eta_{1}, \ldots, \eta_{m} \in \Omega^{1}(M)$, the iterated integral of the word $\eta_{m} \ldots \eta_{1}$ (note the reversed order of symbols) along $\gamma$ is defined by

$$
\int_{\gamma} \eta_{1} \ldots \eta_{m}=\int_{0<t_{1}<\cdots<t_{m}<1} \gamma^{*} \eta_{1}\left(t_{1}\right) \wedge \cdots \wedge \gamma^{*} \eta_{m}\left(t_{m}\right) .
$$

One can show using the calculus of variations [10] that the iterated integral of a linear combination of forms $f=\sum_{I} c_{I} \omega_{i_{1}} \ldots \omega_{i_{m}}$ only depends on the homotopy class of $\gamma$ if and only if the integrability condition (3.8) is satisfied. In this case, an iterated integral varies holomorphically as a function of the endpoint $z=\gamma(1)$ of $\gamma$, and therefore defines a holomorphic function on the universal covering $\widehat{M}$. We can realise $\Omega^{*}\left(\mathcal{O}_{M}\right)$ as an algebra of differential forms on $\widehat{M}$ by taking the pull-back along the covering map $p: \widehat{M} \rightarrow M$. When we refer to a multi-valued function (or form) on $M$ it will be a linear combination of such iterated integrals with coefficients in $\mathcal{O}_{M}$ (resp. $\Omega^{*}\left(\mathcal{O}_{M}\right)$ ) (compare the multi-valued de Rham complex defined in [33]).

Lemma $3.28([32,10,31])$. - Let $\eta_{1}, \ldots, \eta_{l} \in \Omega^{1}(M)$. 
1. Let $1 \leq m \leq l$, and let $\mathfrak{S}(m, l-m)$ denote the set of $(m, l-m)$-shuffles defined in $\S 2.10$. Then the shuffle product formula holds:

$$
\int_{\gamma} \eta_{1} \ldots \eta_{m} \int_{\gamma} \eta_{m+1} \ldots \eta_{l}=\sum_{\sigma \in \mathfrak{S}(m, l-m)} \int_{\gamma} \eta_{\sigma(1)} \ldots \eta_{\sigma(l)} .
$$

2. Let $\gamma_{z}:[0,1] \rightarrow M$ denote a smooth family of paths such that $\gamma_{z}(0)=b$, and $\gamma_{z}(1)=z \in M$. If $\sum_{I} c_{I} \omega_{i_{1}} \ldots \omega_{i_{m}}$ satisfies the integrability condition (3.8), we have:

$$
\frac{d}{d z} \int_{\gamma_{z}} \sum_{I} c_{I} \omega_{i_{m}} \ldots \omega_{i_{1}}=\sum_{I} c_{I} \omega_{i_{1}} \int_{\gamma_{z}} \omega_{i_{m}} \ldots \omega_{i_{2}} .
$$

Definition 3.29. - Let $L_{b}(M)$ denote the $\mathcal{O}_{M}$-module generated by all such homotopyinvariant iterated integrals on $\widehat{M}$. We write $\Omega^{i}\left(L_{b}(M)\right)=L_{b}(M) \otimes_{\mathcal{O}_{M}} \Omega^{i}\left(\mathcal{O}_{M}\right)$.

By the previous lemma, $\Omega^{*}\left(L_{b}(M)\right)$ is a differential algebra, and there is a map:

$$
\begin{aligned}
\rho_{b}: \Omega^{*} B\left(\mathcal{O}_{M}\right) & \stackrel{\sim}{\longrightarrow} \Omega^{*} L_{b}(\widehat{M}) \\
\sum_{I} \phi_{I}\left[\omega_{i_{1}}|\ldots| \omega_{i_{m}}\right] & \longmapsto \sum_{I} \phi_{I} \int_{\gamma} \omega_{i_{m}} \ldots \omega_{i_{1}},
\end{aligned}
$$

which is a surjective map of differential algebras by (3.11). As above, $\gamma$ denotes a smooth path beginning at the point $b \in M$. The kernel of $\rho_{b}$ is a differential ideal, and therefore must reduce to zero since $B\left(\mathcal{O}_{M}\right)$ is differentially simple. Therefore (3.26) is an isomorphism.

Corollary 3.30. - If $\left\{e_{i}\right\}$ is a basis for $B\left(\mathcal{O}_{M}\right)$ over $\mathcal{O}_{M}$, then the functions $\rho_{b}\left(e_{i}\right)$ are linearly independent over $\mathcal{O}_{M}$. All algebraic relations between the functions $\rho_{b}\left(e_{i}\right)$ are determined by the shuffle product.

One can determine a basis for $B\left(\mathcal{O}_{M}\right)$ in the fibre-type case (see $\left.\S 6.2\right)$.

\subsection{Proof of Theorem 3.26}

We first show that the ring of constants of $B\left(\mathcal{O}_{M}\right)$ is $k$. For any $\psi \in B\left(\mathcal{O}_{M}\right), n \geq 0$, we write $\psi_{n}=\operatorname{gr}_{n}^{w} \psi$ for its graded part of weight $n$.

LeMma 3.31. - $H_{\mathrm{DR}}^{0}\left(B\left(\mathcal{O}_{M}\right)\right)=k$.

Proof. - Let $\psi \in B\left(\mathcal{O}_{M}\right)$ of weight $m \geq 1$ such that $d \psi=0$. We write

$$
\psi_{r}=\sum_{I=\left(i_{1}, \ldots, i_{r}\right)} f_{I}\left[\omega_{i_{1}}|\ldots| \omega_{i_{r}}\right] \quad \text { for } 0 \leq r \leq m,
$$

where each $f_{I} \in \mathcal{O}_{M}$. Then the graded weight $m$ part of $d \psi$ is zero:

$$
(d \psi)_{m}=\sum_{|I|=m} d f_{I}\left[\omega_{i_{1}}|\ldots| \omega_{i_{m}}\right]=0 .
$$

Therefore $d f_{I}=0$ and so $f_{I} \in H_{\mathrm{DR}}^{0}\left(\mathcal{O}_{M}\right)=k$ for all ordered sets $I$ such that $|I|=m$. The weight $m-1$ part of $d \psi$ is also zero:

$$
(d \psi)_{m-1}=\sum_{|I|=m} f_{I} \omega_{i_{1}}\left[\omega_{i_{2}}|\ldots| \omega_{i_{r}}\right]+\sum_{J=\left(i_{2}, \ldots, i_{m}\right)} d f_{J}\left[\omega_{i_{2}}|\ldots| \omega_{i_{m}}\right]=0
$$


which implies that

$$
f_{i_{1}, i_{2}, \ldots, i_{m}} \omega_{i_{1}}+d f_{i_{2}, \ldots, i_{m}}=0 \quad \text { for all } i_{2}, \ldots, i_{m} .
$$

Therefore the forms $f_{i_{1}, \ldots, i_{m}} \omega_{i_{1}}$ are exact for all $i_{1}, \ldots, i_{m}$. But since we have shown that $f_{i_{1}, \ldots, i_{m}} \in k$ is constant, this can only occur if $f_{i_{1}, \ldots, i_{m}}=0$. This implies that the weight of $\psi$ is at most $m-1$, which contradicts the initial assumption. Therefore, any $\psi$ such that $d \psi=0$ is of weight 0 , and lies in $\mathcal{O}_{M}$. Hence $\psi \in H_{\mathrm{DR}}^{0}\left(\mathcal{O}_{M}\right)=k$.

The following lemma states that we can replace a closed 1-form in $B\left(\mathcal{O}_{M}\right)$ with an element in its cohomology class of strictly lower weight.

Lemma 3.32. - Let $\psi \in \Omega^{1}\left(B\left(\mathcal{O}_{M}\right)\right)$ be an element of weight $m$ such that $d \psi=0$. Then there exists $\theta \in B\left(\mathcal{O}_{M}\right)$ such that $\kappa=\psi-d \theta$ is of weight at most $m-1$.

Proof. - Let

$$
\psi=\sum_{r=0}^{m} \sum_{I=\left(i_{1}, \ldots, i_{r}\right)} \phi_{I}\left[\omega_{i_{1}}|\ldots| \omega_{i_{r}}\right]
$$

where $\phi_{I} \in \Omega^{1} \mathcal{O}_{M}$ for all indexing sets $I$. Since $d \psi=0$, we deduce that

$$
0=\sum_{|I|=m} d \phi_{I}\left[\omega_{i_{1}}|\ldots| \omega_{i_{m}}\right]-\sum_{|I|=m} \phi_{I} \wedge \omega_{i_{1}}\left[\omega_{i_{2}}|\ldots| \omega_{i_{m}}\right]+\sum_{r=0}^{m-1} d\left(\psi_{r}\right) .
$$

This implies firstly that $d \phi_{I}=0$ for all sets $I$ with $|I|=m$, and secondly that

$$
\sum_{r=0}^{m-1} d\left(\psi_{r}\right)-\sum_{I=\left(i_{1}, \ldots, i_{m}\right)} \phi_{I} \wedge \omega_{i_{1}}\left[\omega_{i_{2}}|\ldots| \omega_{i_{m}}\right]=0 .
$$

Taking the graded part of this equation of weight $m-1$, we deduce that

$$
-\sum_{I=\left(i_{1}, \ldots, i_{m}\right)} \phi_{I} \wedge \omega_{i_{1}}\left[\omega_{i_{2}}|\ldots| \omega_{i_{m}}\right]+\sum_{i_{2}, \ldots, i_{m}} d \phi_{i_{2}, \ldots, i_{m}}\left[\omega_{i_{2}}|\ldots| \omega_{i_{m}}\right]=0
$$

and so

$$
\sum_{i_{1}} \phi_{i_{1}, \ldots, i_{m}} \wedge \omega_{i_{1}}=d \phi_{i_{2}, \ldots, i_{m}}
$$

for all $I=\left(i_{1}, \ldots, i_{m}\right)$. We have shown that $\phi_{I}$ is closed for $|I|=m$, so we can write

$$
\phi_{I}=\sum_{j} \alpha_{I, j} \omega_{j}+d g_{I}
$$

where $\alpha_{I, j} \in k$, and $g_{I} \in \mathcal{O}_{M}$. Substituting into (3.28) above, we have

$$
\sum_{i_{1}, j} \alpha_{i_{1}, \ldots, i_{m}, j} \omega_{j} \wedge \omega_{i_{1}}+\sum_{i_{1}} d g_{i_{1}, \ldots, i_{m}} \wedge \omega_{i_{1}}=d \phi_{i_{2}, \ldots, i_{m}},
$$

for all $i_{2}, \ldots, i_{m}$. The corollary to Theorem 3.1 implies that any linear combination of exterior products of forms $\omega_{i}$ which is exact, is necessarily zero. Using the fact that $d g_{i_{1}, \ldots, i_{m}} \wedge \omega_{i_{1}}=d\left(g_{i_{1}, \ldots, i_{m}} \wedge \omega_{i_{1}}\right)$ is exact, we have

$$
\sum_{i_{1}, j} \alpha_{i_{1}, \ldots, i_{m}, j} \omega_{j} \wedge \omega_{i_{1}}=0, \quad \text { for all } i_{2}, \ldots, i_{m} .
$$


Let

$$
\theta_{1}=\sum_{I=\left(i_{1}, \ldots, i_{m}\right)} \sum_{j} \alpha_{I, j}\left[\omega_{j}\left|\omega_{i_{1}}\right| \ldots \mid \omega_{i_{m}}\right]
$$

Since the integrability condition (3.8) is homogeneous with respect to the weight, the integrability of $\psi$ implies the integrability of $\psi_{m}=\sum_{|I|=m} \phi_{I}\left[\omega_{i_{1}}|\ldots| \omega_{i_{m}}\right]$. This is equivalent to a number of linear equations of the form $\sum_{|I|=m} \lambda_{I} \phi_{I}=0$, where $\lambda_{I} \in k$. Using the decomposition (3.29), and the fact that $\operatorname{Im}\left(\bigoplus_{i<j} k \omega_{i} \wedge \omega_{j} \rightarrow \Omega^{1}\left(\mathcal{O}_{M}\right)\right)$ and $d \mathcal{O}_{M}$ are complementary spaces (this follows from Theorem (3.1)), we deduce that

$$
\sum_{I=\left(i_{1}, \ldots, i_{m}\right)} \sum_{j} \alpha_{I, j} \omega_{j}\left[\omega_{i_{1}}|\ldots| \omega_{i_{m}}\right]
$$

is integrable, as is $\sum_{|I|=m} d g_{I}\left[\omega_{i_{1}}|\ldots| \omega_{i_{m}}\right]$. By adding constants, we can assume that the primitives $g_{I}$ of $d g_{I}$ satisfy the same linear equations $\sum_{|I|=m} \lambda_{I} g_{I}=0$. This ensures that

$$
\theta_{2}=\sum_{|I|=m} g_{I}\left[\omega_{i_{1}}|\ldots| \omega_{i_{m}}\right]
$$

satisfies the integrability criterion also. The integrability of $\theta_{1}$ follows from (3.31) and (3.30). We set $\theta=\theta_{1}+\theta_{2} \in B\left(\mathcal{O}_{M}\right)$. By construction, we have

$$
\begin{aligned}
d \theta-\psi & =d\left(\sum_{I=\left(i_{1}, \ldots, i_{m}\right)} \sum_{j} \alpha_{I, j}\left[\omega_{j}\left|\omega_{i_{1}}\right| \ldots \mid \omega_{i_{m}}\right]+g_{I}\left[\omega_{i_{1}}|\ldots| \omega_{i_{m}}\right]\right)-\psi \\
& =\sum_{|I|=m}\left(\sum_{j} \alpha_{I, j} \omega_{j}+d g_{I}\right)\left[\omega_{i_{1}}|\ldots| \omega_{i_{m}}\right]+g_{I} \wedge \omega_{i_{1}}\left[\omega_{i_{2}}|\ldots| \omega_{i_{m}}\right]-\psi \\
& =\sum_{|I|=m} g_{I} \wedge \omega_{i_{1}}\left[\omega_{i_{2}}|\ldots| \omega_{i_{m}}\right]-\left(\psi_{0}+\cdots+\psi_{m-1}\right),
\end{aligned}
$$

which is of weight at most $m-1$, since all terms of weight $m$ cancel by (3.29).

Given a closed form $\psi \in \Omega^{1}\left(\mathcal{O}_{M}\right)$ of weight $m$, we defined an explicit $\theta \in B\left(\mathcal{O}_{M}\right)$ such that $\psi=d \theta+\psi_{1}$, and $\psi_{1}$ is of weight $\leq m-1$. In fact, $\theta$ is of weight at most $m+1$. Applying the lemma repeatedly, we obtain a series of forms $\psi_{1}, \ldots, \psi_{m} \in \Omega^{1}\left(\mathcal{O}_{M}\right)$ and $\theta_{1}, \ldots, \theta_{m} \in B\left(\mathcal{O}_{M}\right)$, where $\psi_{i}$ is of weight at most $m-i$, such that

$$
\psi_{i}=d \theta_{i}+\psi_{i+1} .
$$

At the final stage, $\psi_{m}=d \theta_{m}$. Thus $\psi=d\left(\theta+\theta_{1}+\cdots+\theta_{m}\right)$, and $\theta+\theta_{1}+\cdots+\theta_{m}$ is a primitive of $\psi$ of weight at most $m+1$.

As remarked earlier, the argument in the proof of the lemma can be both generalised and simplified using spectral sequence arguments (see the appendix).

Corollary 3.33. $-H_{\mathrm{DR}}^{1}\left(B\left(\mathcal{O}_{M}\right)\right)=0$.

This completes the proof of Theorem 3.26. The fact that every $\mathcal{O}_{M}$-subalgebra of $B\left(\mathcal{O}_{M}\right)$ is differentially simple, and the fact that $B\left(\mathcal{O}_{M}\right)$ is a polynomial algebra, follows from the results of $\S 3.3$. 


\subsection{Fibrations of hyperplane arrangements}

We recall necessary and sufficient conditions for an affine hyperplane arrangement to decompose as a fibration over an arrangement of smaller dimension [43]. We deduce from the results of $\S 3.5$ that the reduced bar construction has trivial cohomology for fibre-type arrangements.

Let $\mathfrak{H}=\left\{H_{1}, \ldots, H_{N}\right\}$ denote any affine hyperplane arrangement in $\mathbb{A}^{\ell}$. Choose any affine subspace $W \cong \mathbb{A}^{e}$ contained in $\mathbb{A}^{\ell}$ and let $V_{0} \subset \mathbb{A}^{\ell}$ denote a complementary subspace such that

$$
\mathbb{A}^{\ell} \cong V_{0} \oplus W
$$

For each $z \in \mathbb{A}^{e} \cong W$, let $V_{z}=V_{0}+z$ denote the affine space parallel to $V$ passing through the point $z \in W$. The spaces $V_{z}$ define a vertical direction normal to the base $W$. We define the set of vertical hyperplanes to be

$$
\mathfrak{H}^{v}=\left\{H \in \mathfrak{H}: H \text { contains } V_{z} \text { for some } z \in W\right\},
$$

and let $\mathfrak{H}^{h}$ denote the set of all remaining hyperplanes. There is a decomposition

$$
\mathfrak{H}=\mathfrak{H}^{v} \sqcup \mathfrak{H}^{h},
$$

and it is clear that every horizontal hyperplane $H \in \mathfrak{H}^{h}$ intersects each $V_{z}$ properly. Consider the complements

$$
M=\mathbb{A}^{\ell} \backslash \bigcup_{H \in \mathfrak{H}} H, \quad \text { and } \quad M^{\prime}=W \backslash \bigcup_{H \in \mathfrak{H}^{v}} H \cap W .
$$

The linear projection $\mathbb{A}^{\ell} \rightarrow W$ with kernel $V_{0}$ induces a surjective map $p: M \rightarrow M^{\prime}$.

Lemma 3.34. - The map $p$ is a fibration if and only if the following condition holds: for all $H, H^{\prime} \in \mathfrak{H}$ such that $H \cap H^{\prime} \neq \varnothing$, there exists $H^{\prime \prime} \in \mathfrak{H}^{v}$ such that

$$
H^{\prime \prime} \supseteq H \cap H^{\prime} .
$$

The fibre over $z \in M^{\prime}$ is the complement $V_{z} \backslash \cup_{H \in \mathfrak{H}^{h}}\left(H \cap V_{z}\right)$.

The proof is left as an exercise.

Definition 3.35. - An affine hyperplane arrangement is said to be of fibre-type if it can be expressed as an iterated sequence of linear fibrations whose fibres are of dimension 1. Thus there is a sequence of fibrations

$$
M \stackrel{E_{1}}{\longrightarrow} M_{1}, \quad \ldots, \quad M_{\ell-2} \stackrel{E_{\ell-1}}{\longrightarrow} M_{\ell-1},
$$

where $E_{1}, \ldots, E_{\ell-1}$, and $E_{\ell}=M_{\ell-1}$, are of dimension 1 .

We consider in greater detail the case where the dimension of the fibres is 1 . Then each fibre is isomorphic to $\mathbb{A}^{1}$ minus a finite number of points. Let us choose coordinates compatible with the direct sum decomposition $\mathbb{A}^{\ell}=W \oplus V_{0}$. In other words, let $x_{1}, \ldots, x_{\ell-1}$ denote coordinates on $\mathbb{A}^{\ell-1}=W$, and let $y$ denote the vertical coordinate on $\mathbb{A}^{1}=V_{0}$. Let $\mathcal{O}_{M}$ and $\mathcal{O}_{M^{\prime}}$ denote the rings of regular functions on the affine schemes $M$ and $M^{\prime}$ respectively. Let us write the equations of all horizontal hyperplanes in the form $y-f_{i}=0$, where $f_{i} \in \mathcal{O}_{M^{\prime}}$, 


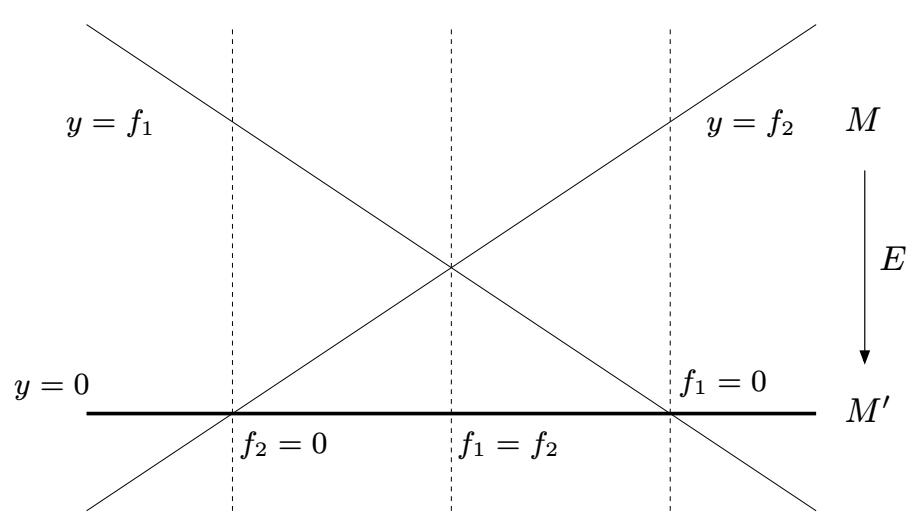

FIgURE 12. An arrangement $M$ in $\mathbb{A}^{\ell}$ which fibres over $M^{\prime} \subset \mathbb{A}^{\ell-1}$ (the thick line at the bottom). A vertical hyperplane (dashed) passes through every point where horizontal hyperplanes intersect.

and $1 \leq i \leq N_{h}$, for some integer $N_{h}$. Thus $H_{i}=\operatorname{ker}\left(y-f_{i}\right)$ for $1 \leq i \leq N_{h}$. By the previous lemma, the fact that $M$ is a fibration is equivalent to the equations (see Figure 12):

$$
\frac{1}{f_{i}-f_{j}} \in \mathcal{O}_{M^{\prime}} \quad \text { for all } \quad 1 \leq i, j \leq N_{h}
$$

We have

$$
\mathcal{O}_{M}=\mathcal{O}_{M^{\prime}}\left[y, \frac{1}{y-f_{1}}, \ldots, \frac{1}{y-f_{N_{h}}}\right] .
$$

We have already shown that the rings $\mathcal{O}_{M}$ and $\mathcal{O}_{M^{\prime}}$ are differentially simple over $k$. We are therefore in the situation considered in $\S 3.5$ (compare (3.16) and (3.17)).

Definition 3.36. - Let us write $\beta_{i}=d y /\left(y-f_{i}\right)$ for $1 \leq i \leq N_{h}$. We define the relative bar construction of $M$ over the base $M^{\prime}$ to be the free $\mathcal{O}_{M}$-shuffle algebra:

$$
B_{M^{\prime}}(E)=\mathcal{O}_{M}\left\langle\beta_{1}, \ldots, \beta_{N_{h}}\right\rangle .
$$

The relative bar construction is a differential $\mathcal{O}_{M}$-algebra with respect to the operator $\partial / \partial y$. Note that since $E$ is of dimension 1, there is no integrability condition. Proposition 3.22 gives:

$$
H^{0}\left(B_{M^{\prime}}(E)\right)=\mathcal{O}_{M^{\prime}}, \text { and } H^{1}\left(B_{M^{\prime}}(E)\right)=0 .
$$

Proposition 3.23 and its corollary imply the following result.

Corollary 3.37. - For each $\mathcal{O}_{M^{\prime}}$-linear map $p: \mathcal{O}_{M} \rightarrow \mathcal{O}_{M^{\prime}}[[\epsilon]][1 / \epsilon]$ which satisfies $p \partial_{y}=\partial_{\epsilon} p$, there is a natural action of the operators $\partial / \partial x_{1}, \ldots, \partial / \partial x_{\ell-1}$ on $B_{M^{\prime}}(E)$, such that the $\partial / \partial x_{i}$ commute with $p$. As a result, $B\left(M^{\prime}\right) \otimes_{\mathcal{O}_{M^{\prime}}} B_{M^{\prime}}(E)$ is the unipotent closure of $\mathcal{O}_{M}$. We deduce that there is an isomorphism of differential $\mathcal{O}_{M}\left[\partial / \partial x_{1}, \ldots, \partial / \partial x_{\ell-1}, \partial / \partial y\right]$ algebras:

$$
B(M) \cong B\left(M^{\prime}\right) \otimes_{\mathcal{O}_{M^{\prime}}} B_{M^{\prime}}(E) .
$$

The following theorem follows by induction. 
THEOREM 3.38. - Let $M$ be a fibre-type affine hyperplane arrangement with fibrations (3.32). There is a (non-unique) isomorphism of differential algebras

$$
B(M) \cong B_{M_{1}}\left(E_{1}\right) \otimes_{\mathcal{O}_{M_{1}}} \cdots \otimes B_{M_{\ell-1}}\left(E_{\ell-1}\right) \otimes_{\mathcal{O}_{M_{\ell-1}}} B\left(E_{\ell}\right) .
$$

COROLlary 3.39. - The de Rham cohomology of the reduced bar construction on a fibretype affine hyperplane arrangement defined over a field $k$ is trivial:

$$
H_{\mathrm{DR}}^{0}(B(M)) \cong k \quad \text { and } \quad H_{\mathrm{DR}}^{i}(B(M))=0 \quad \text { for all } i \geq 1 .
$$

The reason why this result is true is essentially because arrangements of fibre type are rational $K(\pi, 1)$ spaces (see [20], [33]). By (2.3), $\mathfrak{M}_{0, S}$ is a fibre-type affine hyperplane arrangement over $\mathbb{Q}$.

COROLlary 3.40. - In the case of moduli spaces $\mathfrak{M}_{0, S}$ this gives:

$$
H_{\mathrm{DR}}^{0}\left(B\left(\mathfrak{M}_{0, S}\right)\right)=\mathbb{Q}, \quad \text { and } \quad H_{\mathrm{DR}}^{i}\left(B\left(\mathfrak{M}_{0, S}\right)\right)=0 \quad \text { for all } \quad i \geq 1 .
$$

The primitive of a closed form $f \in W^{b} \Omega^{i} B\left(\mathfrak{M}_{0, S}\right)$ is of weight at most $b+1$.

This result can be proved directly using the fact that the hyperplane arrangement $\mathfrak{M}_{0, S}$ is quadratic (see Appendix, Section 9). This is equivalent to Corollary 8.7 in [33], since $\mathfrak{M}_{0, p+2}=Y_{1}^{p}$ in the notation of that paper. The fact that primitives increase the weight by at most one is clear from the definition of the differential (3.11) on $B\left(\mathcal{O}_{M}\right)$.

In the case of the moduli spaces $\mathfrak{M}_{0, S}$, we can make the decomposition of Theorem 3.38 totally canonical by working in cubical coordinates (2.5). The corresponding fibrations are given by the maps $\left(x_{1}, \ldots, x_{\ell}\right) \mapsto\left(x_{1}, \ldots, x_{\ell-1}\right)(\S 2.4)$. Furthermore, there is a base-point at infinity corresponding to the origin, which is compatible with this sequence of fibrations. It is given by the map:

$$
\begin{aligned}
\mathcal{O}\left(\mathfrak{M}_{0, S}\right) \cong \mathbb{Q}\left[\left(x_{i}^{ \pm 1}\right)_{1 \leq i \leq \ell},\left(\frac{1}{1-x_{i} \ldots x_{j}}\right)_{1 \leq i \leq j \leq \ell}\right] & \longrightarrow k\left\{\epsilon_{1}, \ldots, \epsilon_{\ell}\right\} \\
x_{i} & \longmapsto \epsilon_{i} .
\end{aligned}
$$

There is a corresponding logarithmic Laurent expansion over this point, whose map of constants is trivial:

$$
\begin{aligned}
B\left(\mathfrak{M}_{0, S}\right) & \longrightarrow U\left\{\epsilon_{1}, \ldots, \epsilon_{\ell}\right\} \\
\sum_{I=\left(i_{1}, \ldots, i_{m}\right)} c_{I}\left[\omega_{i_{1}}|\ldots| \omega_{i_{m}}\right] & \longmapsto 0 .
\end{aligned}
$$

Because we have fixed a $k\left\{\epsilon_{1}, \ldots, \epsilon_{\ell}\right\}$-point, the isomorphism in Theorem 3.38 is unique.

COROLlaRY 3.41. - In cubical coordinates, there is a canonical isomorphism

$$
B\left(\mathfrak{M}_{0, S}\right) \cong \mathcal{O}\left(\mathfrak{M}_{0, S}\right) \otimes_{\mathbb{Q}} \bigotimes_{k=1}^{\ell} \mathbb{Q}\left\langle\left[d \log x_{k}\right],\left[d \log \left(1-x_{i} \ldots x_{k}\right)\right]_{1 \leq i \leq k}\right\rangle,
$$

where the algebras on the right are free shuffle algebras.

There is a similar decomposition for any set of vertex coordinates $x_{1}^{\alpha}, \ldots x_{\ell}^{\alpha}$, where $\alpha \in \chi_{S, \delta}^{\ell}$ does not contain an internal triangle. 
REMARK 3.42. - In order to compute the periods of $\mathfrak{M}_{0, S}$, we shall only require the fact that $H^{\ell}\left(B\left(\mathfrak{M}_{0, S}\right)\right)=0$, where $\ell=|S|-3$. In cubical coordinates, this is equivalent to finding a primitive to

$$
f d x_{1} \ldots d x_{\ell} \quad \text { for all } \quad f \in B\left(\mathfrak{M}_{0, S}\right) .
$$

We have in fact proved a much stronger result. Corollary 3.41 implies that we can find $F \in B\left(\mathfrak{M}_{0, S}\right)$ such that $\partial F / \partial x_{\ell}=f$. The constant term of $F$ is uniquely determined by the map of constants (3.37). In other words, there is a primitive of the form

$$
F d x_{1} \ldots d x_{\ell-1}
$$

where the weight of $F$ is at most one more than the weight of $f$. The primitive $F$ constructed in this way has the advantage that it is unique.

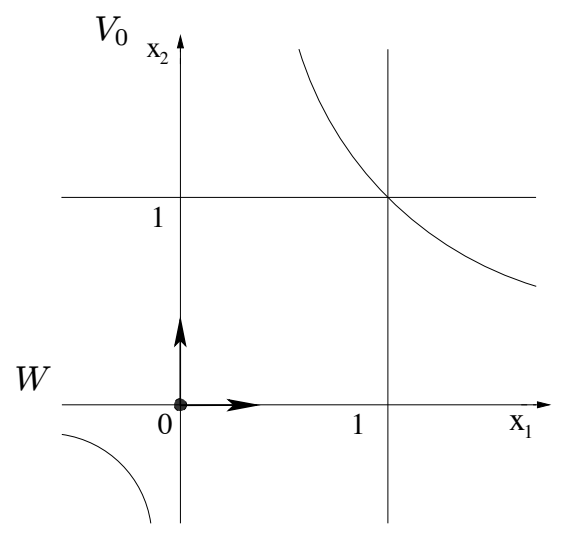

FIGURE 13. In cubical coordinates, there is a natural base point at infinity on $\mathfrak{M}_{0,5}$ corresponding to $(0,0)$.

EXAmple 3.43. - Consider the fibration $\mathfrak{M}_{0,5} \rightarrow \mathfrak{M}_{0,4}$, whose fibres are isomorphic to $\mathbb{A}^{1}$ minus 3 points (fig. 13). In cubical coordinates, we have:

$$
\begin{aligned}
\mathcal{O}_{M^{\prime}} & \cong \mathbb{Q}\left[x, \frac{1}{x}, \frac{1}{1-x}\right], \\
\mathcal{O}_{M} & \cong \mathcal{O}_{M^{\prime}}\left[y, \frac{1}{y}, \frac{1}{1-y}, \frac{x}{1-x y}\right],
\end{aligned}
$$

where the fibration map is the projection onto the $x$-axis:

$$
(x, y) \mapsto x: \mathfrak{M}_{0,5} \rightarrow \mathbb{P}^{1} \backslash\{0,1, \infty\} .
$$

There is a natural $k\left\{\epsilon_{1}, \epsilon_{2}\right\}$-point at the origin which sends

$$
\begin{aligned}
p: \mathcal{O}_{M} & \longrightarrow k\left\{\epsilon_{1}, \epsilon_{2}\right\} \\
x & \longmapsto \epsilon_{1} \\
y & \longmapsto \epsilon_{2},
\end{aligned}
$$

and which maps, for example, $x /(1-x y)$ to $\sum_{i \geq 0} \epsilon_{1}^{i+1} \epsilon_{2}^{i}$. The differential algebra $B\left(M^{\prime}\right)=B\left(\mathbb{P}^{1} \backslash\{0,1, \infty\}\right)$ is the universal algebra of multiple polylogarithms in one 
variable defined in [7], and $B_{M^{\prime}}(E)$ is the relative bar construction over $\mathcal{O}_{M}^{\prime}$. As algebras, each one is the free non-commutative algebra on two (respectively three) symbols:

$$
B\left(M^{\prime}\right)=\mathcal{O}_{M^{\prime}}\left\langle\frac{d x}{x}, \frac{d x}{1-x}\right\rangle, \quad \text { and } \quad B_{M^{\prime}}(E)=\mathcal{O}_{M}\left\langle\frac{d y}{y}, \frac{d y}{1-y}, \frac{x d y}{1-x y}\right\rangle .
$$

Corollary 3.41 gives a canonical isomorphism

$$
B\left(M^{\prime}\right) \otimes_{\mathcal{O}_{M^{\prime}}} B_{M^{\prime}}(E) \stackrel{\sim}{\longrightarrow} B(M),
$$

and enables us to write down a basis of integrable words in $B(M)$. However, the map $B_{M^{\prime}}(E) \rightarrow B(M)$ is far from trivial. For example, it gives

$$
1 \otimes\left[\frac{d y}{1-y} \mid \frac{x d y}{1-x y}\right] \mapsto\left[\frac{d y}{1-y}-\frac{d x}{1-x}-\frac{d x}{x} \mid \frac{x d y+y d x}{1-x y}\right]+\left[\frac{d x}{1-x} \mid \frac{d y}{1-y}\right] .
$$

A similar formula was given in [53] and [32]. It is obvious that the left-hand side is integrable, the right-hand side not so. The left-hand coding can be retrieved from the one on the right by formally setting $d x=0$. The map $B_{M^{\prime}}(E) \rightarrow B(M)$ is canonically normalised in such a way that, apart from all terms of the form $\left[\frac{d y}{y}\right] \amalg \ldots \amalg\left[\frac{d y}{y}\right]$, its image vanishes on setting $d y=y=0$. The logarithmic Laurent expansion of this example is given by the multiple logarithm (see $\S 5.4)$ :

$$
\operatorname{Li}_{1,1}(x, y)=\sum_{0<k<l} \frac{x^{k} y^{l}}{k l},
$$

where we have written $x, y$ instead of $\epsilon_{1}, \epsilon_{2}$. The coding on the left-hand side of the equation above only takes into account the differential equations which $\operatorname{Li}_{1,1}(x, y)$ satisfies with respect to the variable $y$ (which are very simple), the right-hand side encodes the differential equations with respect to both variables $x$ and $y$. The coproduct of $\operatorname{Li}_{1,1}(x, y)$ can be read off the right-hand coding directly:

$$
\begin{aligned}
\Delta \mathrm{Li}_{1,1}(x, y)= & \operatorname{Li}_{1,1}(x, y) \otimes 1+(\log (1-y)-\log (1-x)+\log x) \otimes \log (1-x y) \\
& +\log (1-x) \otimes \log (1-y)+1 \otimes \operatorname{Li}_{1,1}(x, y) .
\end{aligned}
$$

One can compare this with the coproduct for the motivic multiple polylogarithms defined by Goncharov [25].

The integrable words corresponding to the function $\operatorname{Li}_{2,1}(x, y)$ are likewise:

$$
\begin{aligned}
1 \otimes & {\left[\frac{d y}{1-y}\left|\frac{d y}{y}\right| \frac{x d y}{1-x y}\right] \mapsto\left[\frac{d y}{1-y}\left|\frac{d x}{x}+\frac{d y}{y}\right| \frac{x d y+y d x}{1-x y}\right] } \\
& +\left[\frac{d x}{x}\left|\frac{d y}{1-y}-\frac{d x}{1-x}-\frac{d x}{x}\right| \frac{x d y+y d x}{1-x y}\right]+\left[\frac{d x}{x}\left|\frac{d x}{1-x}\right| \frac{d y}{1-y}\right] .
\end{aligned}
$$

We therefore have two different points of view on $B\left(\mathfrak{M}_{0, S}\right)$. On the one hand, there is a direct definition in terms of hyperplane configurations, from which the differential structure and the action of the symmetric group are evident. The problem is that the complexity of the set of integrable words grows rapidly, and the algebraic structure is obscured. On the other hand, using the fibration map above, we have a description of $B\left(\mathfrak{M}_{0, S}\right)$ as a product of free shuffle algebras, from which its algebraic structure is completely evident. But this point of view breaks the symmetry and only part of the differential structure is visible. By exploiting both points of view, one can deduce a lot of information about the structure of $B\left(\mathfrak{M}_{0, S}\right)$. In 
particular, by regarding it as a representation of the symmetric group, one obtains many interesting functional relations between multiple polylogarithms. This will be studied in detail in a future paper.

\section{Manifolds with corners and Fuchsian differential equations}

Let $X$ denote a real analytic manifold with corners. We consider functions on $X$ which have logarithmic singularities along the boundary of $X$, and we define the regularised limit of such a function along components of the boundary $\partial X$. Next, we state and prove a generalised Fuchs theorem in many variables, and show that, in the unipotent case, we obtain solutions on $X$ which are precisely of this type, i.e., which have logarithmic singularities along $\partial X$. Finally, we state a version of Stokes' theorem in the case when $X$ is compact. This requires some regularity results which allow the integration of functions with logarithmic divergences along the boundary of $X$. The example to bear in mind throughout this section is when $X=\bar{X}_{S, \delta}$ is the closed Stasheff polytope defined in $\S 2.7$.

\subsection{Manifolds with corners}

A manifold with corners $X$ is a differentiable manifold whose charts are diffeomorphic to sets of the form

$$
U_{p, q}=\mathbb{R}^{p} \times \mathbb{R}_{+}^{q},
$$

where $\mathbb{R}_{+}=\{x \in \mathbb{R}: x \geq 0\}$, and $p, q \geq 0$ [6]. If $q \geq 1$, the boundary of $U_{p, q}$ is

$$
\partial U_{p, q}=\bigcup_{i+j=q-1} \mathbb{R}^{p} \times \mathbb{R}_{+}^{i} \times\{0\} \times \mathbb{R}_{+}^{j}
$$

which is a union of sets diffeomorphic to $U_{p, q-1}$, and is empty if $q=0$. Let $\partial^{i} U_{p, q}$ denote the successive submanifolds with corners obtained by iteration. There is a stratification

$$
U_{p, q} \supseteq \partial U_{p, q} \supseteq \cdots \supseteq \partial^{q} U_{p, q}
$$

which has the combinatorial structure of a face of a hypercube. There are many different ways to define maps between charts depending on how rigid we wish to make the manifold $X$. We require that derivatives of maps between charts do not vanish along boundary components, and in order for logarithmic regularisation to be well-defined, we must rule out maps of the form $x \mapsto k x: \mathbb{R}_{+} \rightarrow \mathbb{R}_{+}$, where $k \neq 1$.

Definition 4.1. - Let $n=p+q \geq 1$, and let $x_{1}, \ldots, x_{n}$ be coordinates on $\mathbb{R}^{n}$ such that $U_{p, q}=\mathbb{R}^{p} \times \mathbb{R}_{+}^{q}=\left\{x_{1} \geq 0, \ldots, x_{q} \geq 0\right\}$. Let $\mathfrak{S}_{q}$ denote the symmetric group on $q$ letters which permutes the indices $1, \ldots, q$. We define $\operatorname{Hom}_{\mathrm{an}}\left(U_{p, q}, U_{p, q}\right)$ to be the ring of analytic isomorphisms (i.e., whose Jacobian does not vanish anywhere along $U_{p, q}$ ):

$$
\phi=\left(\phi_{1}, \ldots, \phi_{n}\right): U_{p, q} \longrightarrow U_{p, q}
$$

which permute the components of the boundary $\partial U_{p, q}$, i.e.,

$$
\left.\phi_{i}\right|_{x_{\sigma(i)}=0}=0, \quad \text { for } 1 \leq i \leq q,
$$

$4^{\text {e }}$ SÉRIE - TOME $42-2009-\mathrm{N}^{\mathrm{o}} 3$ 
where $\sigma \in \mathfrak{S}_{q}$, and which satisfy

$$
\left.\frac{\partial \phi_{i}}{\partial x_{\sigma(i)}}\right|_{x_{\sigma(i)}=0}=1
$$

In other words, $\phi=\left(x_{\sigma(1)} f_{1}, \ldots, x_{\sigma(q)} f_{q}, \phi_{q+1}, \ldots, \phi_{n}\right)$, where $f_{i}$ are analytic functions such that $f_{i}$ is identically equal to 1 along the boundary component $\left\{x_{\sigma(i)}=0\right\} \subset \partial U_{p, q}$, for $1 \leq i \leq q$. For example, if $n=2$ and $U_{0,2}=\left\{\left(x_{1}, x_{2}\right): x_{1}, x_{2} \geq 0\right\}$, the map $\phi\left(x_{1}, x_{2}\right)=\left(x_{1}+x_{1}^{2} x_{2}, x_{2}+x_{2}^{2}\right)$ is in $\operatorname{Hom}_{\mathrm{an}}\left(U_{0,2}, U_{0,2}\right)$.

We define an analytic manifold with corners to be a manifold with corners whose transition maps lie in $\operatorname{Hom}_{\mathrm{an}}\left(U_{p, q}, U_{p, q}\right)$.

It follows from this definition that any $\phi \in \operatorname{Hom}_{\mathrm{an}}\left(U_{p, q}, U_{p, q}\right)$ preserves the boundary stratification of $U_{p, q}$ (4.1). Any analytic manifold with corners therefore admits a global stratification

$$
X=X_{0} \supseteq X_{1} \supseteq X_{2} \supseteq \cdots \supseteq X_{n},
$$

where each $X_{i}$ is a manifold with corners, and $X_{i+1}=\partial X_{i}$ is the union of the boundary components of $X_{i}$.

Consider the closed Stasheff polytope $X=\bar{X}_{S, \delta}$ contained in $\mathfrak{M}_{0, S}^{\delta}(\mathbb{R})$. Then $X$ is a manifold with corners whose stratification is given by (2.40). To see this, let $0<\varepsilon \ll 1$ denote a small constant, and let $e \in \chi_{S, \delta}^{k}$ denote a $k$-decomposition of the regular $n$-gon $(S, \delta)$, where $k$ can be zero. This can be completed, in a non-unique way, to a full triangulation $\alpha \in \chi_{S, \delta}^{\ell}$. Then $F_{\alpha}$ is a corner contained in the face $F_{e}=\left\{u_{i j}=0:\{i, j\} \in e\right\}$. Define

$$
U_{e, \alpha}(\varepsilon)=\left\{0 \leq u_{i j}<\varepsilon \text { for }\{i, j\} \in e, 0<u_{k l}<1 \text { for }\{k, l\} \in \alpha \backslash e\right\} \subset \mathfrak{M}_{0, S}^{\delta}(\mathbb{R}) .
$$

Since we know that $\left\{u_{i j},\{i, j\} \in \alpha\right\}$ defines a local coordinate system on $\mathfrak{M}_{0, S}^{\delta}(\mathbb{R})$ (Proposition 2.22), $U_{e, \alpha}(\varepsilon)$ is diffeomorphic to a chart $U_{\ell-k, k}=\mathbb{R}^{\ell-k} \times \mathbb{R}_{+}^{k}$ when $\varepsilon$ is sufficiently small. We have (cf. (2.35)):

$$
\bar{X}_{S, \delta}=X_{S, \delta} \cup \bigcup_{k \geq 1} \bigcup_{e \in \chi_{S, \delta}^{k}} U_{e, \alpha}(\varepsilon), \quad \text { for some } \varepsilon>0 .
$$

This proves that $\bar{X}_{S, \delta}$ is indeed an analytic manifold with corners, since all transition maps between boundary components of charts are given by permutations of coordinates. The action of the dihedral group of symmetries on $\bar{X}_{S, \delta}$ is a morphism of analytic manifolds with corners.

\subsection{Logarithmic singularities and regularisation}

We define three sheaves of functions on an analytic manifold with corners $X$ which have singularities along its boundary $\partial X$. They are:

$$
\mathcal{F}^{\text {an }} \subset \mathcal{F}^{\log } \subset \mathcal{F}_{p}^{\log }
$$

where $\mathcal{F}^{\text {an }}$ denotes the sheaf of analytic functions on $X, \mathcal{F}^{\text {log }}$ denotes the sheaf of functions

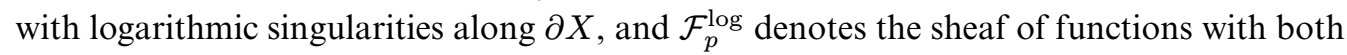
logarithmic singularities and ordinary poles along $\partial X$. 
More precisely, let $p, q \geq 0$ where $n=p+q \geq 1$, and let $x_{1}, \ldots, x_{n}$ be coordinates on $\mathbb{R}^{n}$ such that $U_{p, q}=\mathbb{R}^{p} \times \mathbb{R}_{+}^{q}=\left\{x_{1} \geq 0, \ldots, x_{q} \geq 0\right\}$. Then we define

$$
\mathcal{F}^{\text {an }}\left(U_{p, q}\right) \subset \mathbb{R}\left[\left[x_{1}, \ldots, x_{n}\right]\right],
$$

to be the ring of convergent Taylor series in the variables $x_{1}, \ldots, x_{n}$. Next, we define

$$
\begin{aligned}
& \mathcal{F}^{\log }\left(U_{p, q}\right)=\mathcal{F}^{\text {an }}\left(U_{p, q}\right)\left[\log x_{1}, \ldots, \log x_{q}\right], \\
& \mathcal{F}_{p}^{\log \left(U_{p, q}\right)}=\mathcal{F}^{\text {an }}\left(U_{p, q}\right)\left[x_{1}^{-1}, \ldots, x_{q}^{-1}, \log x_{1}, \ldots, \log x_{q}\right],
\end{aligned}
$$

where $\log x_{i}$ is the principal branch of the logarithm along $\mathbb{R}_{+}$. It follows by a monodromy argument that the functions $\log x_{i}$ are linearly independent over the $\operatorname{ring} \mathcal{F}^{\text {an }}\left(U_{p, q}\right)$. Similar rings of functions in one variable (polynomials in $\log x$ with analytic coefficients) were considered in [42].

For each $1 \leq i \leq n$, let $v_{i}$ denote the valuation map on $\mathcal{F}^{\text {an }}\left(U_{p, q}\right)$ which associates to any function the order of its vanishing along $x_{i}=0$. It extends to a valuation

$$
v_{i}: \mathcal{F}_{p}^{\log }\left(U_{p, q}\right) \longrightarrow \mathbb{Z}
$$

once we have adopted the convention that $v_{i}\left(\log x_{i}\right)=0$.

Lemma 4.2. - Let $X$ denote an analytic manifold with corners. Then $\mathcal{F}^{\text {an }}, \mathcal{F}^{\log }$, and $\mathcal{F}_{p}^{\log }$ define sheaves on $X$, and for each boundary component $D$ of $\partial X$, the valuation map $v_{D}$ on $\mathcal{F}_{p}^{\log }$ is well-defined.

Proof. - Let $\phi \in \operatorname{Hom}_{\mathrm{an}}\left(U_{p, q}, U_{p, q}\right)$. It suffices to check that the composition with $\phi$ preserves $\mathcal{F}^{\log }\left(U_{p, q}\right)$. Let $\phi=\left(\phi_{1}, \ldots, \phi_{n}\right)$. By Definition 4.1, and by permuting the coordinates if necessary, we have

$$
\phi_{i}\left(x_{1}, \ldots, x_{n}\right)=x_{i} f_{i}\left(x_{1}, \ldots, x_{n}\right), \quad \text { for } 1 \leq i \leq q,
$$

where $f_{i} \in \mathcal{F}^{\text {an }}\left(U_{p, q}\right)$. This implies that $f_{i}\left(x_{1}, \ldots, x_{n}\right) \geq 0$ for all $\left(x_{1}, \ldots, x_{n}\right) \in U_{p, q}$, and furthermore, $v_{i}\left(f_{i}\right)=0$. It follows that

$$
\log \left(\phi_{i}\left(x_{1}, \ldots, x_{n}\right)\right)=\log \left(x_{i}\right)+\log \left(f_{i}\left(x_{1}, \ldots, x_{n}\right)\right), \text { for } 1 \leq i \leq q,
$$

where $\log f_{i} \in \mathcal{F}^{\text {an }}\left(U_{p, q}\right)$ is analytic. It follows that $\phi^{*} \mathcal{F}^{\log }\left(U_{p, q}\right) \subset \mathcal{F}^{\log }\left(U_{p, q}\right)$, and, similarly, $\phi^{*} \mathcal{F}_{p}^{\log }\left(U_{p, q}\right) \subset \mathcal{F}_{p}^{\log }\left(U_{p, q}\right)$. The fact that the valuations are well-defined along the components of $\partial X$ follows immediately from (4.4).

We can define the regularised value of a function along boundary components of $X$ by formally setting the functions $\log x_{i}$ to 0 , for $1 \leq i \leq q$, on each chart $U_{p, q}$ of $X$.

Definition 4.3. - Let $f \in \mathcal{F}^{\log }\left(U_{p, q}\right)$, and let $1 \leq l \leq q$. We can write

$$
f=\sum_{I=\left(i_{1}, \ldots, i_{l}\right) \in \mathbb{N}^{l}} f_{I} \log ^{i_{1}} x_{1} \ldots \log ^{i_{l}} x_{l}, \quad \text { where } f_{I} \in \mathcal{F}^{\log }\left(U_{p+l, q-l}\right),
$$

and $f_{I}$ is zero for all but finitely many indices $I$. The regularized value of $f$ along $D=\left\{\left(x_{1}, \ldots, x_{n}\right): x_{1}=\cdots=x_{l}=0\right\} \subset \partial^{l} U_{p, q}$ is defined to be:

$$
\operatorname{Reg}(f, D)=f_{(0, \ldots, 0)}\left(0, \ldots, 0, x_{l+1}, \ldots, x_{q}, x_{q+1}, \ldots, x_{n}\right),
$$

viewed as a function on $D \cong U_{p, q-l}$. By construction, $\operatorname{Reg}(f, D) \in \mathcal{F}^{\log }(D)$. 
Definition-Proposition 4.4. - Let $X$ denote an analytic manifold with corners, and let $D \subset \partial^{l} X$ denote any boundary component of $X$. Then there is a well-defined regularisation map along the component $D$ :

$$
\operatorname{Reg}(\bullet, D): \mathcal{F}^{\log }(X) \longrightarrow \mathcal{F}^{\log }(D) .
$$

Proof. - The transition maps are compatible with logarithmic regularisation by Definition 4.1. Let $\phi \in \operatorname{Hom}_{\mathrm{an}}\left(U_{p, q}, U_{p, q}\right)$. Then, up to permuting coordinates, $\phi$ is of the form $\phi=\left(x_{1}\left(1+x_{1} g_{1}\right), \ldots, x_{q}\left(1+x_{q} g_{q}\right), \phi_{q+1}, \ldots, \phi_{n}\right)$. It follows that

$$
\log \phi_{i}=\log x_{i}+\log \left(1+x_{i} g_{i}\right), \quad \text { for } 1 \leq i \leq q,
$$

and the term $\log \left(1+x_{i} g_{i}\right)$ vanishes at $x_{i}=0$. It follows that logarithmic regularisation along $x_{i}=0$ is well-defined for $1 \leq i \leq q$. By regularising with respect to one variable at a time, it follows that regularisation along an arbitrary boundary component $D \subset \partial^{l} X$ is well-defined also.

Remark 4.5. - Clearly we can extend the regularisation map for polar singularities

$$
\operatorname{Reg}(\bullet, D): \mathcal{F}_{p}^{\log }(X) \longrightarrow \mathcal{F}_{p}^{\log }(D),
$$

by mapping all negative powers of coordinates $x_{1}, \ldots, x_{l}$ to zero also (this is just a map of constants as defined in $\S 3.4$ ).

\subsection{Fuchsian differential equations in several complex variables}

Consider the open complex affine space obtained by complexifying $U_{p, q}$ :

$$
\mathbb{C}^{p+q} \backslash\left\{z_{1} \ldots z_{q}=0\right\}
$$

and let $V_{p, q}$ denote an open polydisk neighbourhood of the origin contained in $\mathbb{C}^{p+q} \backslash\left\{z_{1} \ldots z_{q}=0\right\}$. We require a generalised Fuchs' theorem which we solve locally on the spaces $V_{p, q}$. Let $m \geq 1$, and consider the differential equation:

$$
d F=\Omega F,
$$

where $F$ takes values in the set of $m \times m$ complex matrices $M_{m}(\mathbb{C})$, and where

$$
\Omega=\sum_{i=1}^{p+q} N_{i} \frac{d z_{i}}{z_{i}}+A_{i} d z_{i} .
$$

Here, $N_{i} \in M_{m}(\mathbb{C})$ are constant matrices, and each $A_{i}$ is a holomorphic function on $V_{p, q}$, which takes values in $M_{m}(\mathbb{C})$. Assume that $\Omega$ is integrable, i.e.,

$$
d \Omega=\Omega \wedge \Omega .
$$

This implies, in particular, that the matrices $N_{i}$ commute:

$$
\left[N_{i}, N_{j}\right]=0 \quad \text { for all } \quad 1 \leq i, j \leq n .
$$

Let us write $n=p+q \geq 1$, and suppose that $N_{i}=0$ for all $q+1 \leq i \leq n$. The form $\Omega$ is continuous on $V_{p, q}$. Let us fix branches of the $\operatorname{logarithm} \log z_{i}$ for $i=1, \ldots, q$ on $V_{p, q}$. 
In practice, we will choose a real subspace $\mathbb{R}^{p} \times \mathbb{R}_{+}^{q} \cong U_{p, q} \subset V_{p, q}$ and take the principal branches of the logarithm as previously. The function

$$
D=\exp \left(\sum_{i=1}^{q} N_{i} \log z_{i}\right)
$$

is a well-defined multi-valued function on $V_{p, q}$ because the matrices $N_{i}$ commute. The following result is a generalized Fuchs' theorem in many complex variables. Similar situations have been considered in $[11,52]$.

Theorem 4.6. - Suppose that for each $1 \leq i \leq n$, no pair of eigenvalues of the matrix $N_{i}$ differs by an non-zero integer. Let $H_{0} \in M_{m}(\mathbb{C})$ be any constant matrix. Then (4.5) has a unique solution

$$
F=H D,
$$

where $H: V_{p, q} \rightarrow M_{m}(\mathbb{C})$ is holomorphic and takes the value $H_{0}$ at the origin.

Proof. - The matrix $D$ is invertible, and is a solution to the differential equation

$$
d D=\left(\sum_{i=1}^{n} N_{i} \frac{d z_{i}}{z_{i}}\right) D
$$

It follows that $F=H D$ is a solution of (4.5) if and only if

$$
d H=\sum_{i=1}^{n}\left[N_{i}, H\right] \frac{d z_{i}}{z_{i}}+A_{i} H d z_{i} .
$$

If we write $\partial_{k}=\partial / \partial z_{k}$, then this is equivalent to the set of equations

$$
\partial_{k} H=\left[N_{k}, H\right] \frac{1}{z_{k}}+A_{k} H, \quad \text { for } 1 \leq k \leq n .
$$

A solution $H$ is holomorphic on $V_{p, q}$ if and only if it can be written as a power series

$$
H=\sum_{0 \leq i_{1}, \ldots, 0 \leq i_{n}} H_{\left(i_{1}, \ldots, i_{n}\right)} z_{1}^{i_{1}} \ldots z_{n}^{i_{n}}
$$

where the coefficients $H_{\left(i_{1}, \ldots, i_{n}\right)} \in M_{m}(\mathbb{C})$ satisfy a growth condition. By substituting such a power series expansion into (4.9) and considering the coefficient of $z_{1}^{i_{1}} \ldots z_{n}^{i_{n}}$, we obtain the following recurrence relations:

$$
\left(i_{k}+1-\operatorname{ad}\left(N_{k}\right)\right) H_{\left(i_{1}, \ldots, i_{k}+1, \ldots, i_{n}\right)}=\sum_{0 \leq j_{1} \leq i_{1}, \ldots, 0 \leq j_{n} \leq i_{n}}\left(A_{k}\right)_{\left(j_{1}, \ldots, j_{n}\right)} H_{\left(i_{1}-j_{1}, \ldots, i_{n}-j_{n}\right)}
$$

for each $1 \leq k \leq n$, where $\left(A_{k}\right)_{\left(j_{1}, \ldots, j_{n}\right)}$ are the coefficients in the power series expansion of $A_{k}$. Now consider a matrix $M \in M_{m}(\mathbb{C})$. If we denote the eigenvalues of $M$ by $\alpha_{1}, \ldots, \alpha_{m}$, then the eigenvalues of ad $M$ are $\alpha_{i}-\alpha_{j}$. The assumption on the eigenvalues of $N_{i}$ is therefore equivalent to the invertibility of the operators

$$
\left(m-\operatorname{ad}\left(N_{k}\right)\right) \quad \text { for all } m \in \mathbb{N},
$$

and for each $1 \leq k \leq n$. The operator on the left-hand side of (4.11) is therefore invertible, so we can solve (4.11) iteratively, provided that these equations are compatible. This means that we must show that the two different ways of obtaining $H_{\left(i_{1}, \ldots, i_{k}+1, \ldots, i_{l}+1, \ldots, i_{n}\right)}$ by applying (4.11) first for $k$ and then for $l$, or the other way round, both lead to the same answer. This is

$4^{\text {e }}$ SÉRIE - TOME $42-2009-\mathrm{N}^{\mathrm{o}} 3$ 
equivalent to the integrability of the form $\Omega$. In order to see this, write $\Omega=\sum_{i} \Omega_{i} d z_{i}$, where $\Omega_{i}=R_{i}+A_{i}$, and $R_{i}=N_{i} / z_{i}$, for $1 \leq i \leq n$. The integrability of $\Omega$ and the commutativity of the $R_{i}$ imply the following equations for all $1 \leq i, j \leq n$ :

$$
\begin{aligned}
& \partial_{j} \Omega_{i}=\partial_{i} \Omega_{j}+\left[\Omega_{j}, \Omega_{i}\right], \\
& \partial_{j} R_{i}=\partial_{i} R_{j}+\left[R_{j}, R_{i}\right] .
\end{aligned}
$$

It follows that the expression

$$
\phi_{i j}(M)=\left(\partial_{j} \Omega_{i}+\Omega_{i} \Omega_{j}\right) M-\Omega_{i} M R_{j}-\Omega_{j} M R_{i}+M\left(R_{j} R_{i}-\partial_{j} R_{i}\right)
$$

is symmetric in $i, j$ for all matrices $M \in M_{m}(\mathbb{C})$. But Equations (4.9) are precisely the set of equations $\partial_{i} H=\Omega_{i} H-H R_{i}$ for $1 \leq i \leq n$. It follows, on applying $\partial_{j}$ to each equation, that

$$
\partial_{j} \partial_{i} H=\phi_{i j} H \quad \text { for all } 1 \leq i, j \leq n .
$$

One can check by differentiating a truncated power series expansion for $H$ that the compatibility of the Equations (4.11) up to a given weight is a consequence of the symmetry of the operators $\phi_{k l}$, for all $1 \leq k, l \leq n$. We can therefore solve (4.11) recursively to obtain a solution of (4.8) of the form (4.10). It remains to check that the function $H$ defined in this manner is holomorphic on $V_{p, q}$. Since the series $A_{k}$ for $1 \leq k \leq n$ are holomorphic on the polydisk $V_{p, q}$, there exist constants $r_{1}, \ldots, r_{n}>0$ and a constant $c>0$ such that

$$
\left\|\left(A_{k}\right)_{\left(i_{1}, \ldots, i_{n}\right)}\right\| \leq c r_{1}^{i_{1}} \ldots r_{n}^{i_{n}} \quad \text { for all } 1 \leq k \leq n .
$$

For $m \geq 1$, let $\varepsilon_{m}=\sup _{1 \leq k \leq n}\left\|\left(m-\operatorname{ad} N_{k}\right)\right\|^{-1}$. By the assumption on the eigenvalues of $N_{k}$ and the remarks above, $\varepsilon_{m} \rightarrow 0$ as $m \rightarrow \infty$. It follows from (4.11) that

$$
\left\|H_{\left(i_{1}, \ldots, i_{k}+1, \ldots, i_{n}\right)}\right\| \leq \varepsilon_{i_{k}+1} \sum_{0 \leq j_{l} \leq i_{l}}\left\|\left(A_{k}\right)_{\left(j_{1}, \ldots, j_{n}\right)}\right\|\left\|H_{\left(i_{1}-j_{1}, \ldots, i_{n}-j_{n}\right)}\right\| .
$$

Now let $s_{k}>r_{k}$, for $1 \leq k \leq n$, and let $m$ be sufficiently large such that $\varepsilon_{m} c \prod_{i=1}^{n}\left(\frac{s_{i}}{s_{i}-r_{i}}\right)<1$. Set

$$
e=\sup _{0 \leq i_{1}, \ldots, i_{n} \leq m_{r}} \frac{\left\|H_{\left(i_{1}, \ldots, i_{n}\right)}\right\|}{s_{1}^{i_{1}} \ldots s_{n}^{i_{n}}}<\infty
$$

Let $M \geq m$, and suppose by induction that $\left\|H_{\left(i_{1}, \ldots, i_{n}\right)}\right\| \leq e s_{1}^{i_{1}} \ldots s_{n}^{i_{n}}$ for all $0 \leq i_{1}, \ldots, i_{n} \leq M$. This is true when $M=m$ by the definition of $e$. Then, by applying (4.12), we deduce from (4.13) that

$$
\begin{aligned}
\left\|H_{\left(i_{1}, \ldots, M+1, \ldots, i_{n}\right)}\right\| & \leq \varepsilon_{M+1} \sum_{0 \leq j_{1} \leq i_{1}, \ldots, 0 \leq j_{n} \leq i_{n}} c e\left(\frac{r_{1}}{s_{1}}\right)^{j_{1}} \ldots\left(\frac{r_{n}}{s_{n}}\right)^{j_{n}} s_{1}^{i_{1}} \ldots s_{n}^{i_{n}} \\
& \leq \varepsilon_{M+1} c e \prod_{i=1}^{n}\left(\frac{s_{i}}{s_{i}-r_{i}}\right) s_{1}^{i_{1}} \ldots s_{n}^{i_{n}} \leq e s_{1}^{i_{1}} \ldots s_{n}^{i_{n}} .
\end{aligned}
$$

By induction we deduce that $\left\|H_{\left(i_{1}, \ldots, i_{n}\right)}\right\| \leq e s_{1}^{i_{1}} \ldots s_{n}^{i_{n}}$ for all $\left(i_{1}, \ldots, i_{n}\right)$. This holds for any set of constants $s_{1}, \ldots, s_{n}$ satisfying $s_{i}>r_{i}$, which proves that $H$ is holomorphic on $V_{p, q}$, as required. 
We will be interested in the case where the matrices $N_{i}$ are all nilpotent. It then follows that the matrix

$$
D=\exp \left(\sum_{i=1}^{q} N_{i} \log z_{i}\right)
$$

has coefficients which are polynomials in $\log z_{i}$. Since all eigenvalues of $N_{i}$ are 0 , the condition of the previous theorem is satisfied, and therefore there exists a matrix solution $F$ to Equation (4.5) whose entries $F_{a b}$ are polynomials in $\log z_{1}, \ldots, \log z_{q}$ whose coefficients are convergent Taylor series in $z_{1}, \ldots, z_{n}$ :

$$
F_{a b} \subset \mathbb{C}\left[\left[z_{1}, \ldots, z_{n}\right]\right]\left[\log z_{1}, \ldots, \log z_{q}\right] .
$$

Definition 4.7. - Let $X$ denote an analytic manifold with corners. An integrable 1-form $\Omega$ defined on $X$ is unipotent of Fuchs' type if, locally on each chart of the form $U_{p, q}$, $\Omega$ restricts to a 1 -form of type (4.6), where the matrices $N_{i}$ are nilpotent.

As remarked in $\S 4.2$, there are canonical branches of the functions $\log z_{i}$ on local charts of $X$. The solutions to (4.5) will therefore be real-valued on $X$.

COROLlary 4.8. - Let $\Omega$ be a real-valued unipotent integrable 1-form of Fuchs' type on $X$. Suppose that $X$ is simply connected. Then any solution $\left(F_{a b}\right)$ to (4.5) defined in the neighbourhood of any point $x \in X$ extends over the whole of the interior of $X$. This gives a global solution of (4.5) whose coefficients satisfy $F_{a b} \in \Gamma\left(X, \mathcal{F}^{\log }\right)$.

\subsection{Stokes' theorem with logarithmic singularities}

The key argument in our proof of the main theorem is to apply a version of Stokes' theorem to the manifold with corners $\bar{X}_{S, \delta}$. This requires integrating functions which have logarithmic singularities along the boundary.

Lemma 4.9. - Let $X$ denote a compact analytic manifold with corners of dimension $n$. Let $\psi \in \Omega^{n}(X)$ denote an $n$-form on $X$ whose coefficients lie in $\mathcal{F}_{p}^{\log }$. Then $\psi$ is absolutely integrable on $X$ if and only if $\psi$ has no poles along $\partial X$.

Proof. - If $\psi$ has a pole of order $k \geq 1$ along some component of $\partial X$, then there is a chart on $X$ of the form $U_{p, 1}$ such that $\psi=f d x_{1} \ldots d x_{n}$, where $f$ can be written

$$
f\left(x_{1}, \ldots, x_{n}\right)=\frac{1}{x_{1}^{k}} \sum_{i=0}^{N} f_{i}\left(x_{2}, \ldots, x_{n}\right) \log ^{i} x_{1}+\frac{1}{x_{1}^{k-1}} \sum_{i=0}^{M} g_{i}\left(x_{1}, \ldots, x_{n}\right) \log ^{i} x_{1},
$$

where $f_{i}, g_{i} \in \mathcal{F}^{\text {an }}\left(U_{p, q}\right)$ are analytic on $x_{1}>0, \ldots, x_{n}>0$, and $f_{N}$ is not identically zero. Since the term $\left(\log x_{1}\right)^{N}$ dominates the other powers of $\log x_{1}$ near $x_{1}=0$, it follows by continuity that there is a small box

$$
B(\varepsilon)=\left\{\left(x_{1}, \ldots, x_{n}\right): x_{1} \in[0, \varepsilon], x_{2}-\alpha_{2}, \ldots, x_{n}-\alpha_{n} \in[-\varepsilon, \varepsilon]\right\},
$$

where $\alpha_{2}, \ldots, \alpha_{n}>0$, and a constant $c>0$ such that

$$
|f| \geq \frac{c}{x_{1}^{k}}\left|\log x_{1}\right|^{N}
$$

$4{ }^{\mathrm{e}}$ SÉRIE - TOME $42-2009-\mathrm{N}^{\mathrm{o}} 3$ 
for all $\left(x_{1}, x_{2}, \ldots, x_{n}\right) \in B(\varepsilon)$ whenever $\varepsilon>0$ is sufficiently small. It follows that

$$
\int_{X}|f| d x_{1} \ldots d x_{n} \geq c(2 \varepsilon)^{n-1} \int_{0}^{\varepsilon} \frac{1}{x_{1}^{k}}\left|\log x_{1}\right|^{N} d x_{1}=\infty
$$

and therefore $\psi$ is not absolutely integrable.

Now suppose that $\psi$ has no poles along $\partial X$. Then in each small chart of the form $U_{p, q}$, we can write $\psi=f\left(x_{1}, \ldots, x_{n}\right) d x_{1} \ldots d x_{n}$, where

$$
f\left(x_{1}, \ldots, x_{n}\right)=\sum_{I=\left(i_{1}, \ldots, i_{q}\right)}\left(\log x_{1}\right)^{i_{1}} \ldots\left(\log x_{q}\right)^{i_{q}} f_{I}\left(x_{1}, \ldots, x_{n}\right)
$$

where $f_{I}\left(x_{1}, \ldots, x_{n}\right) \in \mathcal{F}^{\text {an }}\left(U_{p, q}\right)$, and almost all $f_{I}$ are identically zero. But the function $\log x$ is integrable on any interval $[0, t)$, where $t>0$, and since sums and products of integrable functions are integrable, it follows that $f$ is integrable locally. Since $X$ is compact, we can find a finite partition of unity on $X$, and deduce that $f$ is absolutely integrable over the whole of $X$.

We can therefore integrate functions which have at most logarithmic singularities. The following lemma implies that primitives of functions on $X$ which have at most logarithmic singularities extend continuously to $\partial X$. The essential point is that the 1 -form $\log x d x$ on $\mathbb{R}_{+}$has a logarithmic singularity at 0 , but its primitive, $x \log x-x+c$, is continuous at $x=0$.

Lemma 4.10. - Let $X$ be an analytic manifold with corners. Let $\psi \in \Omega^{n}(X)$ have at most logarithmic singularities along $\partial X$, and let $\Psi \in \Omega^{n-1}(X)$ denote a primitive of $\psi$ which has no poles along $\partial X$. Then $\Psi$ is continuous on the interior of $\partial X$.

Proof. - It suffices to prove the result on each chart of $X$ isomorphic to $U_{p, q}$ with coordinates $x_{1}, \ldots, x_{n}$ as above. Let $\psi=f d x_{1} \ldots d x_{n}$, where $f \in \mathcal{F}^{\log }\left(U_{p, q}\right)$. We write $\Psi=\sum_{i=1}^{n}(-1)^{i-1} F_{i} d x_{1} \ldots \widehat{d x}_{i} \ldots d x_{n}$, where $F_{i} \in \mathcal{F}^{\log }\left(U_{p, q}\right)$ for $1 \leq i \leq n$. Let

$$
F_{i}=\sum_{k \geq 0} \log ^{k} x_{i} F_{i, k}
$$

where $F_{i, k} \in \mathcal{F}^{\log }\left(U_{p, q}\right)$ is analytic in the coordinate $x_{i}$ and is zero for all but finitely many indices $k$. Since $\sum_{i=1}^{n} \partial F_{i} / \partial x_{i}=f$, we have

$$
\sum_{i=1}^{n} \sum_{k \geq 1} \frac{k \log ^{k-1} x_{i}}{x_{i}} F_{i, k}\left(x_{1}, \ldots, x_{i-1}, 0, x_{i+1}, \ldots, x_{n}\right) \in \mathcal{F}^{\log }\left(U_{p, q}\right) .
$$

This implies that $F_{i, k}\left(x_{1}, \ldots, x_{i-1}, 0, x_{i+1}, \ldots, x_{n}\right)=0$ for all $1 \leq i \leq n, k \geq 1$, and therefore $F_{i} \in \mathbb{C}\left[\log x_{1}, \ldots, x_{i} \log x_{i}, \ldots, \log x_{q}\right]\left[\left[x_{1}, \ldots, x_{n}\right]\right]$. It follows that

$$
\left.\Psi\right|_{x_{i}=0}=\left.(-1)^{i-1} F_{i}\right|_{x_{i}=0} d x_{1} \ldots \widehat{d x}_{i} \ldots d x_{n}
$$

is continuous for all $1 \leq i \leq n$. Thus $\Psi$ is continuous along the interior of $\partial X$.

We can now state the following version of Stokes' theorem. 
THEOREM 4.11. - Let $X$ denote a compact analytic manifold with corners of dimension $n$. Let $\psi \in \Omega^{n}(X)$ be an $n$-form such that $\psi$ has no poles along $\partial X$, and let $\Psi \in \Omega^{n-1}(X)$ be a primitive of $\psi$ such that $\Psi$ has no poles along $\partial X$ either. Then $\Psi$ extends continuously to $\partial X$, and

$$
\int_{X} \psi=\int_{\partial X} \Psi
$$

where both integrals are finite.

Proof. - Let $U_{p, q}(\varepsilon)=\mathbb{R}^{p} \times \mathbb{R}_{\varepsilon}^{q}$ where $\mathbb{R}_{\varepsilon}=\{x \in \mathbb{R}: x \geq \varepsilon\}$. By Lemma $4.9, \psi$ is integrable on $X$. We know that $\Psi$ extends continuously to $\partial X$ by the previous lemma. On each small chart of $X$ we can apply Stokes' theorem:

$$
\int_{U_{p, q}} \psi=\lim _{\varepsilon \rightarrow 0} \int_{U_{p, q}(\varepsilon)} \psi=\lim _{\varepsilon \rightarrow 0} \int_{\partial U_{p, q}(\varepsilon)} \Psi=\int_{\partial U_{p, q}} \Psi,
$$

and all terms are finite. Since $X$ is compact, we can find a finite partition of unity and apply the above identity locally. The result then follows in exactly the same way as the usual proof of Stokes' theorem.

In the case which interests us, when $X=\bar{X}_{S, \delta}$, we can define the following exhaustion of the polytopes $\bar{X}_{S, \delta}$. For all small $\varepsilon>0$, we set

$$
\bar{X}_{S, \delta}^{\varepsilon}=\left\{u_{i j} \geq \varepsilon, \quad\{i, j\} \in \chi_{S, \delta}\right\} .
$$

The required version of Stokes' theorem is then immediate:

$$
\int_{X_{S, \delta}} \psi=\lim _{\varepsilon \rightarrow 0} \int_{\bar{X}_{S, \delta}^{\varepsilon}} \psi=\lim _{\varepsilon \rightarrow 0} \int_{\partial \bar{X}_{S, \delta}^{\varepsilon}} \Psi=\int_{\partial \bar{X}_{S}} \Psi .
$$

\section{Hyperlogarithms}

We give an explicit description of the constructions in the previous two sections when the dimension is 1 , i.e., when $M$ is the affine line $\mathbb{A}^{1}$ minus $N+1$ fixed points $\sigma_{0}, \ldots, \sigma_{N}$. However, we need to consider iterated integrals whose path of integration has endpoints at one of the removed points $\sigma_{i}$, and so does not necessarily converge. This requires a regularisation procedure which can be solved for all iterated integrals simultaneously by considering their generating series.

\subsection{Hyperlogarithms and differential equations}

Let $N \geq 1$, and let $A=\left\{a_{0}, \ldots, a_{N}\right\}$ be an alphabet with $N+1$ letters. We fix any injective map of sets $j: A \hookrightarrow \mathbb{C}$, and set $\sigma_{0}=j\left(a_{0}\right), \ldots, \sigma_{N}=j\left(a_{N}\right)$. Let $\Sigma$ denote the set $j(A) \cup\{\infty\}$, and let $D=\mathbb{P}^{1}(\mathbb{C}) \backslash \Sigma$ denote the complex plane with the points $\sigma_{k}$ removed. Consider the following formal differential equation:

$$
\frac{\partial}{\partial z} F(z)=\sum_{i=0}^{N} \frac{a_{i}}{z-\sigma_{i}} F(z),
$$

$4^{\mathrm{e}}$ SÉRIE - TOME $42-2009-\mathrm{N}^{\circ} 3$ 
which is an equation of Fuchs type, whose singularities are simple poles in $\Sigma$. Let $F(z)$ be a solution on $D$ taking values in $\mathbb{C}\langle\langle A\rangle\rangle$. If we write

$$
F(z)=\sum_{w \in A^{*}} F_{w}(z) w
$$

then (5.1) is equivalent to the system of equations

$$
\frac{\partial}{\partial z} F_{a_{k} w}(z)=\frac{F_{w}(z)}{z-\sigma_{k}}
$$

for all $0 \leq k \leq N$ and all $w \in A^{*}$, together with the initial equation $\partial F_{1}(z) / \partial z=0$, where 1 denotes the empty word in $A^{*}$. The term $F_{1}(z)$ is therefore constant.

One can construct explicit holomorphic solutions $L_{w}(z)$ to (5.2) on a certain domain $U$ obtained by cutting $\mathbb{C}$. These functions extend by analytic continuation to multi-valued functions on the punctured plane $D$, and can equivalently be regarded as holomorphic functions on a universal covering space $p: \widehat{D} \rightarrow D$. Since no confusion arises, we shall always denote these functions by the same symbol $L_{w}(z)$. For each $0 \leq k \leq N$, choose closed halflines $\ell\left(\sigma_{k}\right) \subset \mathbb{C}$ starting at $\sigma_{k}$, such that no two intersect. Let $U=\mathbb{C} \backslash \bigcup_{\sigma_{k} \in \Sigma} \ell\left(\sigma_{k}\right)$ be the simply-connected open subset of $\mathbb{C}$ obtained by cutting along these half-lines. Fix a branch of $\log \left(z-\sigma_{0}\right)$ on $\mathbb{C} \backslash \ell\left(\sigma_{0}\right)$.

Proposition 5.1. - Equation (5.1) has a unique solution L(z) on U such that

$$
L(z)=f_{0}(z) \exp \left(a_{0} \log \left(z-\sigma_{0}\right)\right)
$$

where $f_{0}(z)$ is a holomorphic function on $\mathbb{C} \backslash \bigcup_{k \neq 0} \ell\left(\sigma_{k}\right)$ which satisfies $f_{0}\left(\sigma_{0}\right)=1$. We write this $L(z) \sim\left(z-\sigma_{0}\right)^{a_{0}}$ as $z \rightarrow \sigma_{0}$. Furthermore, every solution of (5.1) which is holomorphic on $U$ can be written $L(z) C$, where $C \in \mathbb{C}\langle\langle X\rangle\rangle$ is a constant series (i.e., depending only on $\Sigma$, and not on $z$ ).

The proposition can be deduced from Theorem 4.6, and a direct solution is given in Gonzalez-Lorca's thesis [28]. We use another approach here, since we require an explicit formula for the functions $L_{w}(z)$ which is originally due to Poincaré and Lappo-Danilevsky [40, 44]. First, let $A_{c}^{*}$ denote the subset of all words in $A^{*}$ which do not end in the letter $a_{0}$, and let $\mathbb{C}\left\langle A_{c}\right\rangle \subset \mathbb{C}\langle A\rangle$ denote the sub-vector space they generate. It is easy to verify that $\mathbb{C}\left\langle A_{c}\right\rangle$ is preserved by the shuffle product. If $w \in A_{c}^{*}$ and $w \neq 1$, the limiting condition given in the proposition is just $\lim _{z \rightarrow \sigma_{0}} L_{w}(z)=0$. If we write $w=a_{0}^{n_{r}} a_{i_{r}} a_{0}^{n_{r-1}} a_{i_{r-1}} \ldots a_{0}^{n_{1}} a_{i_{1}}$, where $1 \leq i_{1}, \ldots, i_{r} \leq N$, then $L_{w}(z)$ is defined in a neighbourhood of $\sigma_{0}$ by the formula

$$
\sum_{1 \leq m_{1}<\cdots<m_{r}} \frac{(-1)^{r}}{m_{1}^{n_{1}+1} \ldots m_{r}^{n_{r}+1}}\left(\frac{z-\sigma_{0}}{\sigma_{i_{1}}-\sigma_{0}}\right)^{m_{1}}\left(\frac{z-\sigma_{0}}{\sigma_{i_{2}}-\sigma_{0}}\right)^{m_{2}-m_{1}} \cdots\left(\frac{z-\sigma_{0}}{\sigma_{i_{r}}-\sigma_{0}}\right)^{m_{r}-m_{r-1}}
$$

which converges absolutely for $\left|z-\sigma_{0}\right|<\inf \left\{\left|\sigma_{i_{1}}-\sigma_{0}\right|, \ldots,\left|\sigma_{i_{r}}-\sigma_{0}\right|\right\}$. One can easily check that this defines a family of holomorphic functions satisfying the Equations (5.2) in this open disk, and that the limiting condition is trivially satisfied.

The functions $L_{w}(z)$ extend analytically to the whole of $U$ by the recursive integral formula:

$$
L_{a_{k} w}(z)=\int_{\sigma_{0}}^{z} \frac{L_{w}(t)}{t-\sigma_{k}} d t
$$


which is valid for all $0 \leq k \leq N$ and all $w \in A_{c}^{*}$. Since iterated integrals are homomorphisms for the shuffle product (Lemma 3.28), we also have

$$
L_{w}(z) L_{w^{\prime}}(z)=L_{w \amalg w^{\prime}}(z) \quad \text { for all } \quad w, w^{\prime} \in A_{c}^{*},
$$

where $L$ is extended by linearity to all words $w \in \mathbb{C}\left\langle A_{c}\right\rangle$. It follows from the definition of the shuffle product that any word in $A^{*}$ can be uniquely written as a linear combination of shuffles of $a_{0}^{n}$ with words in $A_{c}^{*}$ :

$$
w=\sum_{n \geq 0} a_{0}^{n} \amalg v_{n}, \text { where } v_{n} \in \mathbb{C}\left\langle A_{c}\right\rangle .
$$

We can therefore set

$$
L_{a_{0}}(z)=\log \left(z-\sigma_{0}\right)
$$

and extend the definition of $L_{w}(z)$ to all words $w \in A^{*}$ by demanding that $L_{w}(z)$ satisfy the shuffle relations $L_{w}(z) L_{w}^{\prime}(z)=L_{w ш w^{\prime}}(z)$ for all $w, w^{\prime}$ in $A^{*}$. One verifies that the functions $L_{w}(z)$ can be written in the form (5.4) for all words $w \in A^{*}$, for $w \neq a_{0}^{n}$, and are solutions to (5.2). In order to prove that $f_{0}(z)=L(z) \exp \left(-a_{0} \log \left(z-\sigma_{0}\right)\right)$ is holomorphic at $z=\sigma_{0}$, we use the following lemma.

Lemma 5.2. $-\quad \sum_{i=0}^{n}(-1)^{i} w a_{0}^{n-i} w a_{0}^{i} \equiv 0 \bmod \mathbb{C}\left\langle A_{c}^{*}\right\rangle \quad$ for all $w \in A_{c}^{*}$.

Proof. - Let $\widetilde{\partial}_{a_{0}}$ denote the truncation operator with respect to the letter $a_{0}$ defined in $\S 3.1$, but which acts by truncation on the right, i.e., $\widetilde{\partial}_{a_{0}} w a_{i}=\delta_{0 i} w$, where $\delta_{0 i}$ is the Kronecker delta. It is a derivation with respect to $\amalg$. If we apply it to the left-hand side of the equation, we obtain zero, by the Leibniz formula. This implies that the left-hand side is a linear combination of words not ending in $a_{0}$.

REMARK 5.3. - The operators $\widetilde{\partial}_{a_{i}}$ are related to the 'dérivations étrangères' defined by Écalle [19].

Using the fact that $a_{0}^{\text {mi }}=i ! a_{0}^{i}$, we have

$$
f_{0}(z)=L(z) \exp \left(-a_{0} \log \left(z-\sigma_{0}\right)\right)=\sum_{w \in A^{*}} L_{w}(z) w \sum_{i \geq 0}(-1)^{i} a_{0}^{i} L_{a_{0}^{i}}(z) .
$$

It follows from the previous lemma and the shuffle relations for the functions $L_{w}(z)$, that the coefficient of each word $w a_{0}^{n}$, where $w \in A_{c}^{*}$ and $n \geq 0$, is a linear combination of $L_{w^{\prime}}(z)$, where $w^{\prime} \in A_{c}^{*}$. These are holomorphic at $z=\sigma_{0}$ by construction, and this proves the regularity condition for $f_{0}(z)$.

In order to prove the uniqueness statement in the proposition, let $K(z)$ be any other solution of (5.1) which is holomorphic on $U$. The series $L(z)$ defined above is invertible, as its leading coefficient is the constant function 1 . Let $F(z)=L(z)^{-1} K(z)$. On differentiating the equation $K(z)=L(z) F(z)$, we obtain

$$
\sum_{i=0}^{N} \frac{a_{i}}{z-\sigma_{i}} K(z)=\sum_{i=0}^{N} \frac{a_{i}}{z-\sigma_{i}} L(z) F(z)+L(z) F^{\prime}(z)
$$

by (5.1), and therefore $L(z) F^{\prime}(z)=0$. Since $L(z)$ is invertible, $F^{\prime}(z)=0$, and so $F(z)$ is constant. This completes the proof of the proposition.

$4^{\text {e }}$ SÉRIE - TOME $42-2009-\mathrm{N}^{\mathrm{o}} 3$ 
REMARK 5.4. - The functions $L_{w}(z)$ are known as hyperlogarithms and were originally defined by Poincaré and Lappo-Danilevsky. They were recently resurrected by Aomoto $[1,2,3]$, Écalle [19], and Goncharov [24, 25, 26]. It is clear that $L_{1}(z)=1$, and

$$
\begin{aligned}
& L_{a_{i}^{n}}(z)=\frac{1}{n !} \log ^{n}\left(\frac{z-\sigma_{i}}{\sigma_{0}-\sigma_{i}}\right) \quad \text { if } \quad i \geq 1, \\
& L_{a_{0}^{n}}(z)=\frac{1}{n !} \log ^{n}\left(z-\sigma_{0}\right),
\end{aligned}
$$

for all $n \in \mathbb{N}$. Note that $L_{a_{0}^{n}}(z)$ depends on the choice of branch of $\log \left(z-\sigma_{0}\right)$ which was fixed previously, but that the functions $L_{a_{i}^{n}}(z)$ do not. They are the unique branches which satisfy the limiting condition $L_{a_{i}^{n}}\left(\sigma_{0}\right)=0$.

Given a branch of $\log \left(z-\sigma_{k}\right)$ on $\mathbb{C} \backslash \ell\left(\sigma_{k}\right)$ for each $1 \leq k \leq N$, we obtain by symmetry a solution to (5.1) corresponding to each singularity.

Corollary 5.5. - For every $0 \leq k \leq N$, there exists a unique solution $L^{\sigma_{k}}(z)$ of Equation (5.2) on $U$ such that

$$
L^{\sigma_{k}}(z)=f_{k}(z) \exp \left(a_{k} \log \left(z-\sigma_{k}\right)\right)
$$

where $f_{k}(z)$ is holomorphic on $\mathbb{C} \backslash \bigcup_{i \neq k} \ell\left(\sigma_{i}\right)$ and satisfies $f_{k}\left(\sigma_{k}\right)=1$.

The quotient of any two such solutions is a constant non-commutative series known as a regularised zeta series. Using these series, one can determine the monodromy of hyperlogarithms explicitly ([8]).

\subsection{The bar construction on $\mathbb{P}^{1} \backslash \Sigma$}

In this situation, the variant of the bar construction defined in $\S 3$ is very easy to describe. Let $k$ denote any subfield of $\mathbb{C}$ which contains $\sigma_{0}, \ldots, \sigma_{N}$. The ring of regular functions on $\mathbb{P}^{1} \backslash \Sigma$ is simply

$$
\mathcal{O}_{\Sigma}=k\left[z,\left(\frac{1}{z-\sigma_{j}}\right)_{0 \leq j \leq N}\right]
$$

Since $\mathbb{P}^{1} \backslash \Sigma$ is of dimension one, the integrability condition is trivially satisfied. Let $A^{\vee}=\left\{\psi_{0}, \ldots, \psi_{N}\right\}$, where $\psi_{i}=d \log \left(z-\sigma_{i}\right)$, for $0 \leq i \leq N$. The cohomology classes of the forms $\psi_{i}$ form a $k$-basis for $H^{1}\left(\mathbb{P}^{1} \backslash \Sigma\right)$. Clearly $\psi_{i} \wedge \psi_{j}=0$ for all $0 \leq i, j \leq N$, and therefore $B\left(\mathbb{P}^{1} \backslash \Sigma\right)$ is a shuffle algebra

$$
B\left(\mathbb{P}^{1} \backslash \Sigma\right)=\mathcal{O}_{\Sigma} \otimes_{k} k\left\langle A^{\vee}\right\rangle,
$$

equipped with the derivation

$$
d=\frac{d}{d z} \otimes 1+\sum_{i=0}^{N}\left(\frac{1}{z-\sigma_{i}}\right) \otimes \partial_{\psi_{i}},
$$

where the truncation operators $\partial_{\psi_{i}}$ were defined in $\S 3.1$. Let $L\left(\mathbb{P}^{1} \backslash \Sigma\right)$ denote the $\mathcal{O}_{\Sigma}$-algebra generated by the coefficients of a solution $L$ to (5.1). The analogue of the map (3.26) is the differential homomorphism:

$$
\begin{aligned}
\rho: B\left(\mathbb{P}^{1} \backslash \Sigma\right) & \longrightarrow L\left(\mathbb{P}^{1} \backslash \Sigma\right) \\
w & \longmapsto L_{w}(z),
\end{aligned}
$$


which is the identity on $\mathcal{O}_{\Sigma}$. Theorem 3.26 implies that this map is an isomorphism.

Corollary 5.6. - The functions $L_{w}(z)$, for $w \in A^{*}$, are linearly independent over $\mathcal{O}_{\Sigma}$. Every function in $L\left(\mathbb{P}^{1} \backslash \Sigma\right)$ has a primitive which is unique up to a constant.

The construction of the functions $L_{w}(z)$ used a decomposition of $B\left(\mathbb{P}^{1} \backslash \Sigma\right)$ into convergent and non-convergent parts. This used the fact that the map

$$
(u \otimes v \mapsto u \amalg v): \mathbb{Z}\left\langle A_{c}\right\rangle \otimes \mathbb{Z}\left\langle a_{0}\right\rangle \rightarrow \mathbb{Z}\langle A\rangle
$$

is an isomorphism of algebras. We can therefore define

$$
B_{\sigma_{0}}\left(\mathbb{P}^{1} \backslash \Sigma\right)=\mathcal{O}_{\Sigma}\left\langle A_{c}\right\rangle
$$

to be the sub-algebra of convergent iterated integrals (indexed by words not ending in $a_{0}$ ). It is a differential algebra for the derivation $d$ defined in (5.7). We have

$$
B\left(\mathbb{P}^{1} \backslash \Sigma\right) \cong B_{\sigma_{0}}\left(\mathbb{P}^{1} \backslash \Sigma\right) \otimes_{k\left[z, 1 /\left(z-\sigma_{0}\right)\right]} B\left(\mathbb{P}^{1} \backslash\left\{\sigma_{0}, \infty\right\}\right) .
$$

There is a corresponding decomposition

$$
\left.L\left(\mathbb{P}^{1} \backslash \Sigma\right)\right)=L_{\sigma_{0}}\left(\mathbb{P}^{1} \backslash \Sigma\right) \otimes_{k\left[z, 1 /\left(z-\sigma_{0}\right)\right]} L\left(\mathbb{P}^{1} \backslash\left\{\sigma_{0}, \infty\right\}\right),
$$

where $L\left(\mathbb{P}^{1} \backslash\left\{\sigma_{0}, \infty\right\}\right) \cong k\left[z, 1 /\left(z-\sigma_{0}\right), \log \left(z-\sigma_{0}\right)\right]$. Correspondingly, the hyperlogarithm realisation (5.8) decomposes as a product $\rho=\rho_{\sigma_{0}} \otimes \rho^{\prime}$, where

$$
\rho_{\sigma_{0}}(w)=\int_{\sigma_{0}}^{z} w, \quad \text { for all } w \in A_{c} .
$$

This is a convergent iterated integral, even though the base point $\sigma_{0}$ does not lie in the space $\mathbb{P}^{1} \backslash \Sigma$. The logarithmic divergences are completely determined by the realisation $\rho^{\prime}: B\left(\mathbb{P}^{1} \backslash\left\{\sigma_{0}, \infty\right\}\right) \rightarrow L\left(\mathbb{P}^{1} \backslash\left\{\sigma_{0}, \infty\right\}\right)$, where $\rho^{\prime}\left(a_{0}\right)=\log \left(z-\sigma_{0}\right)$.

REMARK 5.7. - In general, the points $\sigma_{0}, \ldots, \sigma_{N}$ will not be arranged symmetrically. In this case, one needs to do a genuine analytic continuation of the functions $L_{w}(z)$, since the formula (5.3) is not valid outside its radius of convergence. Lappo-Danilevsky described a technique for dealing with this situation, which is described in [8]. This extra complication will not arise in the present context.

\subsection{Quotients of the hyperlogarithm equation}

Now we shall consider the case where the coefficients $a_{i}$ in (5.1) satisfy relations. Therefore, let $A=\left\{a_{0}, \ldots, a_{N}\right\}$ be an alphabet with $N+1$ letters as before, and consider an ideal

$$
I \subset \mathbb{C}\left\langle a_{0}, \ldots, a_{N}\right\rangle .
$$

Typically, $I$ will be generated by commutators of the form $\left[a_{i}, a_{j}\right]$ for $i \neq j$. It defines a closed ideal we also denote by $I$ in the completed algebra $\mathbb{C}\langle\langle A\rangle\rangle$. Let

$$
\pi: \mathbb{C}\langle\langle A\rangle\rangle \longrightarrow \mathbb{C}\langle\langle A\rangle\rangle / I
$$

denote the quotient map. Consider the analogue of Equation (5.1):

$$
\frac{\partial}{\partial z} F(z)=\sum_{i=0}^{N} \frac{\pi\left(a_{i}\right)}{z-\sigma_{i}} F(z)
$$


where, this time, $F$ takes values in the quotient ring $\mathbb{C}\langle\langle A\rangle\rangle / I$. An equation of this type will be called a hyperlogarithm quotient equation.

COROLlary 5.8. - There exists a unique solution $F$ to the hyperlogarithm quotient equation (5.10) with solutions in $\mathbb{C}\langle\langle A\rangle\rangle / I$ such that $F(z) \sim\left(z-\sigma_{0}\right)^{\pi\left(a_{0}\right)}$ as $z \rightarrow \sigma_{0}$.

Proof. - The existence follows immediately from Proposition 5.1, on applying $\pi$ to a solution of (5.1). The uniqueness is proved in the same way.

Now let $L(I)$ denote the $\mathcal{O}_{\Sigma}$-module of functions generated by the coefficients of a solution $F$ to (5.10). It is a differential submodule of $L\left(\mathbb{P}^{1} \backslash \Sigma\right)$. More precisely,

$$
L(I) \cong \mathcal{O}_{\Sigma} \otimes(\mathbb{C}\langle A\rangle / I)^{\vee} \subset \mathcal{O}_{\Sigma} \otimes(\mathbb{C}\langle A\rangle)^{\vee} \cong L\left(\mathbb{P}^{1} \backslash \Sigma\right) .
$$

It follows that the coefficients of solutions to (5.10) are linear combinations of hyperlogarithms. If $I$ is a Hopf ideal, i.e., $\Gamma I \subset 1 \otimes I+I \otimes 1$, then $L(I)$ is an algebra by duality. Theorem 3.26 immediately implies the following corollary.

Corollary 5.9. - Suppose that I is a Hopf ideal. In this case, $L(I)$ is a unipotent extension of $\mathcal{O}_{\Sigma}$. In particular, it is a differentially simple polynomial algebra over $\mathcal{O}_{\Sigma}$ whose ring of constants is $k$.

As an example, consider the equation:

$$
\frac{d F}{d z}=\left(\frac{a_{0}}{z}+\frac{a_{1}}{z-1}\right) F
$$

on $\mathbb{P}^{1} \backslash\{0,1, \infty\}$, and let $I \subset \mathbb{C}\left\langle a_{0}, a_{1}\right\rangle$ denote the ideal generated by $\left[a_{0}, a_{1}\right]$. Then $F=\exp \left(a_{0} \log z+a_{1} \log (z-1)\right)$ is the unique solution satisfying $F \sim \exp \left(a_{0} \log z\right)$ as $z \rightarrow 0$. The differential algebra $L(I)$ is just $\mathbb{C}[z, 1 / z, 1 /(z-1), \log z, \log (z-1)]$.

\subsection{Multiple polylogarithms and hyperlogarithms}

We recall the definition of the multiple polylogarithm functions, which were defined by Goncharov $[23,24,25,26]$. Let $n_{1}, \ldots, n_{r} \in \mathbb{N}$, and consider the power series

$$
\operatorname{Li}_{n_{1}, \ldots, n_{r}}\left(z_{1}, \ldots, z_{r}\right)=\sum_{0<k_{1}<\cdots<k_{r}} \frac{z_{1}^{k_{1}} \ldots z_{r}^{k_{r}}}{k_{1}^{n_{1}} \ldots k_{r}^{n_{r}}},
$$

which converges absolutely for $\left|z_{i}\right| \leq 1$ if $n_{r} \geq 2$ and for $\left|z_{i}\right|<1$ in general.

Now let $\ell \geq 2, x_{1}, \ldots, x_{\ell-1} \in \mathbb{C}$, and set $\Sigma=\left\{\sigma_{0}, \ldots, \sigma_{\ell}, \infty\right\}$, where

$$
\sigma_{0}=0, \quad \sigma_{1}=1, \quad \text { and } \quad \sigma_{i}=\left(x_{\ell-i+1} \ldots x_{\ell-1}\right)^{-1} \quad \text { for } \quad 2 \leq i \leq \ell .
$$

Let $A=\left\{a_{0}, \ldots, a_{\ell}\right\}$ as previously, and let $w=a_{0}^{n_{r}-1} a_{i_{r}} \ldots a_{0}^{n_{1}-1} a_{i_{1}} \in \mathbb{C}\langle A\rangle$, where $1 \leq i_{1}, \ldots, i_{r} \leq \ell$. We suppose that the points $\sigma_{i}$ are distinct and finite (compare (2.5)). Let us consider the points $x_{1}, \ldots, x_{\ell-1}$ as being fixed, and let $x_{\ell} \in \mathbb{P}^{1} \backslash \Sigma$ denote a free variable. By (5.3), the coefficients of the corresponding hyperlogarithm function with respect to $x_{\ell}$, are given near $x_{\ell}=0$ by the formula

$$
\begin{aligned}
L_{w}\left(x_{\ell}\right) & =\sum_{1 \leq m_{1}<\cdots<m_{r}} \frac{(-1)^{r}}{m_{1}^{n_{1}} \ldots m_{r}^{n_{r}}}\left(x_{j_{1}} \ldots x_{\ell}\right)^{m_{1}}\left(x_{j_{2}} \ldots x_{\ell}\right)^{m_{2}-m_{1}} \ldots\left(x_{j_{r}} \ldots x_{\ell}\right)^{m_{r}-m_{r-1}} \\
& =(-1)^{r} \operatorname{Li}_{n_{1}, \ldots, n_{r}}\left(\frac{x_{j_{1}} \ldots x_{\ell}}{x_{j_{2}} \ldots x_{\ell}}, \ldots, \frac{x_{j_{r-1}} \ldots x_{\ell}}{x_{j_{r}} \ldots x_{\ell}}, x_{j_{r}} \ldots x_{\ell}\right),
\end{aligned}
$$


where we have set $j_{k}=\ell-i_{k}+1$ for $1 \leq k \leq r$. It follows that such a multiple polylogarithm, considered as a function of the single variable $x_{\ell}$, is a hyperlogarithm function on $\mathbb{P}^{1} \backslash \Sigma$. The relation between the multiple polylogarithm viewed as a hyperlogarithm in $x_{\ell}$, and the multiple polylogarithm viewed as a function of all its variables, is given by the fibration sequence between moduli spaces $\mathfrak{M}_{0, n}(\S 6.5)$.

\subsection{Multiple zeta values and $\mathbb{P}^{1} \backslash\{0,1, \infty\}$}

In the case where $\sigma_{0}=0$ and $\sigma_{1}=1, D$ is the projective line minus three points. Since $\mathbb{P}^{1} \backslash\{0,1, \infty\}$ also coincides with $\mathfrak{M}_{0,4}$, it is natural to make a change of sign and define $X=\left\{x_{0}, x_{1}\right\}$, where $x_{0}=a_{0}$ and $x_{1}=-a_{1}$. Let $\log z$ denote the principle branch of the logarithm. By Proposition (5.1), the equations

$$
\begin{aligned}
\frac{d L(z)}{d z} & =\left(\frac{x_{0}}{z}+\frac{x_{1}}{1-z}\right) L(z) \\
L(z) & \sim \exp \left(x_{0} \log z\right)
\end{aligned}
$$

have a unique solution $L(z) \in \mathbb{C}\langle\langle X\rangle\rangle$, known as the generating series of multiple polylogarithms in one variable. Its coefficients are written $\operatorname{Li}_{w}(z)$, for $w \in X^{*}$. We have $\mathrm{Li}_{x_{0}}(z)=\log z$ and $\operatorname{Li}_{x_{1}}(z)=-\log (1-z)$. Now consider a word $w \in x_{0} X^{*} x_{1}$ which begins in $x_{0}$ and ends in $x_{1}$. It can be written

$$
w=x_{0}^{n_{r}-1} x_{1} x_{0}^{n_{r-1}-1} x_{1} \ldots x_{0}^{n_{1}-1} x_{1}
$$

where $n_{r} \geq 2$. Equation (5.3) therefore gives a power series expansion:

$$
\operatorname{Li}_{w}(x)=\operatorname{Li}_{n_{1}, \ldots, n_{r}}(1, \ldots, 1, z)=\sum_{0<k_{1}<\cdots<k_{r}} \frac{z^{k_{r}}}{k_{1}^{n_{1}} \ldots k_{r}^{n_{r}}},
$$

which is regular at $z=1$. The numbers $\operatorname{Li}_{w}(1)$ satisfy the shuffle relations by (5.5).

Definition 5.10. - Let $w \in x_{0} X^{*} x_{1}$ as above. The multiple zeta value of weight $n_{1}+\cdots+n_{r}$ and depth $r$ is the real number defined by the convergent sum:

$$
\zeta(w)=\zeta\left(n_{1}, \ldots, n_{r}\right)=\operatorname{Li}_{w}(1)=\sum_{0<k_{1}<\cdots<k_{r}} \frac{1}{k_{1}^{n_{1}} \ldots k_{r}^{n_{r}}}, \quad n_{r} \geq 2 .
$$

The function $\zeta$ extends by linearity to the $\mathbb{Q}$-vector space spanned by $x_{0} X^{*} x_{1}$. We define $\mathcal{Z}$ to be the $\mathbb{Q}$-module generated by the set of all multiple zeta values:

$$
\mathcal{Z}=\mathbb{Q}\left[\zeta(w): w \in x_{0} X^{*} x_{1}\right] .
$$

Because the multiple zeta values satisfy the shuffle relation $\zeta\left(w \omega w^{\prime}\right)=\zeta(w) \zeta\left(w^{\prime}\right)$, and because $\mathbb{Q}\left[w: w \in x_{0} X^{*} x_{1}\right]$ is stable under the shuffle product, $\mathcal{Z} \subset \mathbb{R}$ is an algebra. It is naturally filtered by the weight [51].

It is not difficult to verify that every word $w \in X^{*}$ is a linear combination of shuffles of $x_{0}, x_{1}$ and words $\eta \in x_{0} X^{*} x_{1}$. The map $w \mapsto \zeta(w)$ extends to a unique function on $\mathbb{Z}\langle X\rangle$ which satisfies

$$
\begin{aligned}
& \zeta_{\mathrm{II}}\left(x_{0}\right)=0, \quad \zeta_{\mathrm{II}}\left(x_{1}\right)=0, \\
& \zeta_{\mathrm{II}}\left(w \amalg w^{\prime}\right)=\zeta_{\mathrm{II}}(w) \zeta_{\mathrm{m}}\left(w^{\prime}\right), \quad \text { for all } w, w^{\prime} \in X^{*} .
\end{aligned}
$$


Definition 5.11. - The Drinfeld associator [18] is the non-commutative series

$$
Z^{0,1}=\sum_{w \in X^{*}} \zeta_{\mathrm{m}}(w) w \in \mathcal{Z}\langle\langle X\rangle\rangle
$$

It follows that Drinfeld's associator is precisely the regularised value of $L(z)$ at 1 :

$$
\operatorname{Reg}(L(z), 1)=Z^{0,1} .
$$

\section{The universal algebra of polylogarithms on $\mathfrak{M}_{0, n}$}

In this section, we give an explicit construction of the algebra of all homotopy-invariant iterated integrals on $\mathfrak{M}_{0, S}$ in terms of multiple polylogarithms. By decomposing this algebra as a tensor product of hyperlogarithm algebras, we compute its monodromy in terms of multiple zeta values.

\subsection{The cohomology ring of $\mathfrak{M}_{0, S}$}

Recall that $\mathfrak{M}_{0, S}$ was defined as the quotient of the configuration space of $n=|S|$ distinct points $\left(z_{s}\right)_{s \in S} \in\left(\mathbb{P}^{1}\right)^{S}$, modulo the action of $\mathrm{PSL}_{2}$. Let $i, j, k, l \in S$. The cross-ratio $[i j \mid k l]:\left(\mathbb{P}^{1}\right)_{*}^{S} \rightarrow \mathbb{P}^{1}$ defines a function on $\left(\mathbb{P}^{1}\right)_{*}^{S}$, and since $[i j \mid k l]=1-[i k \mid j l]$, we have:

$$
d \log [i j \mid k l] \wedge d \log [i k \mid j l]=0 .
$$

We introduce the notation

$$
\Delta_{i j}=d \log \left(z_{i}-z_{j}\right)=\frac{d z_{i}-d z_{j}}{z_{i}-z_{j}}, \quad \text { for all } \quad 1 \leq i<j \leq n,
$$

where $\Delta_{i j}=\Delta_{j i}$, and $\Delta_{i i}=0$, for all $1 \leq i, j \leq n$. Equation (6.1) gives a quadratic relation between the $\Delta_{i j}$, which can be simplified as follows. Since $\mathrm{PSL}_{2}(\mathbb{C})$ acts transitively on the projective line $\mathbb{P}^{1}(\mathbb{C})$, and since the cross-ratio is invariant under its action, we can place the point $z_{1}$ at infinity, and it follows that

$$
\mathfrak{M}_{0, S}(\mathbb{C})=\mathbb{C}_{*}^{n-1} / B
$$

where $\mathbb{C}_{*}^{n-1}$ denotes the set of distinct $n-1$-tuples $z_{2}, \ldots, z_{n} \in \mathbb{C}$, and $B \cong \mathbb{C}^{\times} \ltimes \mathbb{C}$ is the subgroup of $\mathrm{PSL}_{2}(\mathbb{C})$ which stabilizes $\infty$. The projection map $\mathbb{C}_{*}^{n-1} \rightarrow \mathfrak{M}_{0, S}(\mathbb{C})$ is a trivial fibration with fibres isomorphic to $B$, and it follows that

$$
H^{\star}\left(\mathbb{C}_{*}^{n-1}\right) \cong H^{\star}\left(\mathfrak{M}_{0, S}(\mathbb{C})\right) \otimes H^{\star}(B)
$$

We can therefore deduce the cohomology of $\mathfrak{M}_{0, S}(\mathbb{C})$ from the structure of $H^{\star}\left(\mathbb{C}_{*}^{n-1}\right)$, which can be described as follows. We apply (6.1) with $l=1$. Using the fact that $z_{1}=\infty$, we deduce that $d \log [i j \mid k l]=\Delta_{i k}-\Delta_{j k}$, and $d \log [i k \mid j l]=\Delta_{i j}-\Delta_{k j}$, viewed as 1 -forms on $\mathbb{C}_{*}^{n-1}$. Then (6.1) yields Arnold's relation:

$$
\Delta_{i j} \wedge \Delta_{j k}+\Delta_{j k} \wedge \Delta_{k i}+\Delta_{k i} \wedge \Delta_{i j}=0,
$$

for any distinct indices $2 \leq i, j, k \leq n$.

THEOREM 6.1 (Arnold [4]). $-H^{\star}\left(\mathbb{C}_{*}^{n-1}\right)$ is the quotient of the free exterior algebra generated by $\Delta_{i j}$ for $2 \leq i, j \leq n$, by the quadratic relations (6.5). 
Now let us fix a dihedral structure $\delta$ on $S$. In $\S 2$ we defined 1-forms

$$
\omega_{i j}=d \log u_{i j}, \quad \text { for }\{i, j\} \in \chi_{S, \delta} .
$$

Their cohomology classes $\left[\omega_{i j}\right]$ form a basis for $H^{1}\left(\mathfrak{M}_{0, S}(\mathbb{C})\right)$. Recall the definition of $N$ (§3.2) as the kernel of the exterior product:

$$
N=\operatorname{ker}\left(\wedge: H^{1}\left(\mathfrak{M}_{0, S}(\mathbb{C})\right) \otimes H^{1}\left(\mathfrak{M}_{0, S}(\mathbb{C})\right) \longrightarrow H^{2}\left(\mathfrak{M}_{0, S}(\mathbb{C})\right)\right)
$$

Proposition 6.2. - $N$ is spanned by the following elements:

$$
\left(\sum_{\{i, j\} \in A}\left[\omega_{i j}\right]\right) \otimes\left(\sum_{\{k, l\} \in B}\left[\omega_{k l}\right]\right)
$$

where $A, B \subset \chi_{S, \delta}$ are any two sets of chords which cross completely (§2.2). The cohomology ring $H^{\star}\left(\mathfrak{M}_{0, S}(\mathbb{C})\right)$ is isomorphic to the free exterior algebra generated by $\left[\omega_{i j}\right]$, for $\{i, j\} \in \chi_{S, \delta}$, modulo the image of elements of the form (6.6).

Proof. - First we regard each dihedral coordinate $u_{i j}$ as a function on $\left(\mathbb{P}^{1}\right)_{*}^{n}$. By the defining equations (2.10), we have $1-\prod_{a \in A} u_{a}=\prod_{b \in B} u_{b}$ for all sets of chords $A, B \subset \chi_{S, \delta}$ which cross completely. This implies that $d \log \prod_{A} u_{a} \wedge d \log \prod_{B} u_{b}=0$, which is precisely

$$
\left(\sum_{\{i, j\} \in A}\left[\omega_{i j}\right]\right) \wedge\left(\sum_{\{k, l\} \in B}\left[\omega_{k l}\right]\right)=0 .
$$

Furthermore, every instance of (6.1) occurs in this way, since each cross ratio $[i j \mid k l]$ can be written as a product $\prod_{A} u_{a}$ or its inverse, by Lemma 2.2. We now place $z_{1}=\infty$ as above, and view the corresponding relations on $\mathbb{C}_{*}^{n-1}$. By Arnold's theorem, this implies that (6.6) generates the set of all relations on $\mathbb{C}_{*}^{n-1}$. In particular, (6.6) generates $N$, since by $(6.4), H^{\star}\left(\mathfrak{M}_{0, S}(\mathbb{C})\right) \subset H^{\star}\left(\mathbb{C}_{*}^{n-1}\right)$ and so any relation satisfied by the $\omega_{i j}$ is also satisfied in $H^{\star}\left(\mathbb{C}_{*}^{n-1}\right)$.

Now, since $B \cong \mathbb{C}^{\times} \ltimes \mathbb{C}$ is homotopy equivalent to a circle, $H^{*}(B)$ is the exterior algebra generated by a single cohomology class which we denote $\beta \in H^{1}(B)$. It follows from (6.4) that $H^{*}\left(\mathfrak{M}_{0, S}(\mathbb{C})\right)$ is the subalgebra of $H^{*}\left(\mathbb{C}^{n-1}\right)$ of degree 0 in $\beta$. We deduce from Arnold's theorem that $H^{*}\left(\mathfrak{M}_{0, S}(\mathbb{C})\right)$ is the quotient of the free exterior algebra generated by a basis of $H^{1}\left(\mathfrak{M}_{0, S}(\mathbb{C})\right)$, modulo $N$.

Similar results have been obtained by Getzler [22].

REMARK 6.3. - The quadratic relations (6.5) are equivalent to the existence of the dilogarithm function, in the following sense. Let $f=[i j \mid k l]$. Then identity (6.5) is precisely the integrability of the element

$$
[d \log f \mid d \log (1-f)] \in W^{2} B\left(\mathfrak{M}_{0, S}\right) .
$$

The iterated integral ( $\S 3.6$ ) corresponding to this element is the function $\operatorname{Li}_{2}(f)$.

$4^{\text {e }}$ SÉRIE - TOME $42-2009-\mathrm{N}^{\mathrm{o}} 3$ 


\subsection{The universal algebra of polylogarithms on $\mathfrak{M}_{0, S}$}

Recall that in simplicial coordinates (2.3), the space $\mathfrak{M}_{0, S}$ is the open complement of an affine hyperplane arrangement. Its ring of regular functions is

$$
\mathcal{O}\left(\mathfrak{M}_{0, S}\right)=\mathbb{Q}\left[u_{i j}, u_{i j}^{-1}\right] \cong \mathbb{Q}\left[\left(t_{i}\right)_{1 \leq i \leq \ell},\left(\frac{1}{t_{i}}\right)_{1 \leq i \leq \ell},{\frac{1}{\left(1-t_{i}\right)}}_{1 \leq i \leq \ell}, \frac{1}{\left(t_{i}-t_{j}\right)}{ }_{1 \leq i<j \leq \ell}\right],
$$

which is a differential algebra with respect to the partial differential operators $\partial / \partial t_{i}$. We defined the abstract algebra of homotopy-invariant iterated integrals on $\mathfrak{M}_{0, S}$ using the reduced bar construction in $\S 3.2$.

Definition 6.4. - The universal algebra of polylogarithms on $\mathfrak{M}_{0, S}$ is the differential graded algebra $B\left(\mathfrak{M}_{0, S}\right)=B\left(\mathcal{O}\left(\mathfrak{M}_{0, S}\right)\right)$.

Recall that $B\left(\mathfrak{M}_{0, S}\right)$ is the unipotent closure of $\mathcal{O}\left(\mathfrak{M}_{0, S}\right)$, and that its de Rham cohomology is trivial, i.e., $H_{\mathrm{DR}}^{0}\left(B\left(\mathfrak{M}_{0, S}\right)\right) \cong \mathbb{Q}$, and $H_{\mathrm{DR}}^{i}\left(B\left(\mathfrak{M}_{0, S}\right)\right)=0$ for all $i \geq 1$. The structure of $B\left(\mathfrak{M}_{0, S}\right)$ is particularly rich: it has a natural Hopf algebra structure over $\mathcal{O}\left(\mathfrak{M}_{0, S}\right)$, and also carries an action of the symmetric group $\mathfrak{S}(S)$ by functoriality. The graded pieces of the set of indecomposable elements in $B\left(\mathfrak{M}_{0, S}\right)$ of fixed weight yield very interesting finitedimensional representations of $\mathfrak{S}(S)$. Correspondingly, there is an action by the subgroup of dihedral symmetries $D_{2 n}$ of the $n$-gon $(S, \delta)$. This action is evident from the symmetric description of $H^{1}\left(\mathfrak{M}_{0, S}\right)$ and $N$ in terms of the forms $\omega_{i j}$, for $\{i, j\} \in \chi_{S, \delta}$, given in Proposition 6.2.

Now if we pass to cubical coordinates, we can split $B\left(\mathfrak{M}_{0, S}\right)$ as a tensor product of shuffle algebras, and subsequently decompose it into convergent and non-convergent pieces. First, recall that we defined a base point at infinity (3.37), corresponding to the origin $x_{1}=\cdots=x_{\ell}=0$, which is locally a normal crossing divisor. The base point is given by the map $\mathcal{O}\left(\mathfrak{M}_{0, S}\right) \rightarrow k\left\{\epsilon_{1}, \ldots, \epsilon_{\ell}\right\}$ which maps $x_{i}$ to $\epsilon_{i}$, for $1 \leq i \leq \ell$. The projection map $\left(x_{1}, \ldots, x_{\ell}\right) \mapsto\left(x_{1}, \ldots, x_{\ell-1}\right)$ defines a linear fibration $\mathfrak{M}_{0,\left\{s_{1}, \ldots, s_{n}\right\}} \rightarrow \mathfrak{M}_{0,\left\{s_{2}, \ldots, s_{n}\right\}}$, which forgets the point marked $s_{1}$. In the notations of $\S 2.4$, it corresponds to the choice of sets $T_{1}=\left\{s_{n}, s_{1}, s_{2}, s_{3}\right\}, T_{2}=\left\{s_{2}, s_{3}, \ldots, s_{n-1}\right\}$, and $T_{1} \cap T_{2}=\left\{s_{2}, s_{3}\right\}$. By iterating in this manner, we obtain a sequence of fibrations: $\mathfrak{M}_{0,\left\{s_{i}, \ldots, s_{n}\right\}} \longrightarrow \mathfrak{M}_{0,\left\{s_{i+1}, \ldots, s_{n}\right\}}$, obtained by forgetting the marked point $s_{i}$, for $i=1, n, n-1, \ldots, 4$. By applying Theorem 3.38 to these fibrations, we deduce that there is a canonical isomorphism:

$$
B\left(\mathfrak{M}_{0, S}\right) \cong \bigotimes_{i=1}^{n-3} B_{\mathfrak{M}_{0, \Sigma_{i}}}\left(\mathbb{P}^{1} \backslash \Sigma_{i}\right),
$$

where $\Sigma_{i}=\left\{s_{2}, s_{3} \ldots, s_{n-i+1}\right\}$. Each algebra $B\left(\mathbb{P}^{1} \backslash \Sigma_{i}\right)$ is a universal algebra of hyperlogarithms, and is a free shuffle algebra on $n-i-1$ generators by $\S 5.2$.

CoRollary 6.5. - $B\left(\mathfrak{M}_{0, S}\right)$ is isomorphic, as a $\mathcal{O}\left(\mathfrak{M}_{0, S}\right)$-algebra, to the tensor product of the free shuffle algebras on $2,3, \ldots, n-2$ generators.

Using results of Radford, one can write down a basis of any free shuffle algebra in terms of Lyndon words (see [46]). The corollary implies that a basis of $B\left(\mathfrak{M}_{0, S}\right)$ is given by tensor products of Lyndon words. 
We can now decompose each component of (6.7) into convergent and non-convergent parts. We can define the subalgebra $B_{\mathfrak{M}_{0, \Sigma_{i}}}^{\prime}\left(\mathbb{P}^{1} \backslash \Sigma_{i}\right) \subset B_{\mathfrak{M}_{0, \Sigma_{i}}}\left(\mathbb{P}^{1} \backslash \Sigma_{i}\right)$ of convergent words in a similar manner to (5.9). We can then define

$$
B_{0}\left(\mathfrak{M}_{0, S}\right)=\mathcal{O}\left(\mathfrak{M}_{0, S}\right) \otimes \bigotimes_{i=1}^{n-3} B_{\mathfrak{M}_{0, \Sigma_{i}}}^{\prime}\left(\mathbb{P}^{1} \backslash \Sigma_{i}\right)
$$

Then $B\left(\mathfrak{M}_{0, S}\right)$ decomposes as a commutative tensor product

$$
B\left(\mathfrak{M}_{0, S}\right) \cong B_{0}\left(\mathfrak{M}_{0, S}\right) \otimes_{\mathbb{Q}} \mathbb{Q}\left[\left[d \log x_{1}\right], \ldots,\left[d \log x_{\ell}\right]\right] .
$$

The algebra on the right is the free commutative (polynomial) algebra on generators $\left[\omega_{2} i+3\right]=\left[d \log x_{i}\right]$ for $i=1, \ldots, \ell$.

Lemma 6.6. - The subalgebra $B_{0}\left(\mathfrak{M}_{0, S}\right) \subset B\left(\mathfrak{M}_{0, S}\right)$ is generated as a vector space by the set of integrable words, no element of which ends in a symbol $\omega_{2 k}$, for $4 \leq k \leq n$.

Proof. - Let $A$ denote the $\mathcal{O}\left(\mathfrak{M}_{0, S}\right)$-subalgebra of $B\left(\mathfrak{M}_{0, S}\right)$ generated by the set of all integrable words (i.e. which satisfy (3.8))

$$
\sum_{I} c_{I}\left[\omega_{i_{1} j_{1}}|\ldots| \omega_{i_{r} j_{r}}\right], \quad c_{I} \in \mathbb{Q},
$$

where $\left\{i_{r}, j_{r}\right\} \notin\{\{2,4\}, \ldots,\{2, n\}\}$. It is clear that $A \subset B_{0}\left(\mathfrak{M}_{0, S}\right)$ is a differential subalgebra. Furthermore, one easily checks that every element $a \in \Omega^{1}\left(\mathfrak{M}_{0, S}\right) \otimes_{\mathcal{O}_{\mathfrak{M}_{0, S}}} A$ of weight at least 1 has a primitive in $A$. This follows from the proof of Theorem 3.26 or the argument given in the appendix, since taking primitives involves adding symbols to the left of each word. Using the techniques of $\S 3$, Proposition 3.12, it follows immediately that the map $A \rightarrow B_{0}\left(\mathfrak{M}_{0, S}\right)$ is surjective.

Likewise, for every vertex $v \in V^{\delta}$, the set of vertex coordinates at $v$ defines a base point at infinity, and (by considering the action of the differential Galois group of $U\left\{\epsilon_{1}, \ldots, \epsilon_{\ell}\right\}$ over $k\left\{\epsilon_{1}, \ldots, \epsilon_{\ell}\right\}$, for example), one defines a subalgebra of convergent words $B_{v, \delta}\left(\mathfrak{M}_{0, S}\right)$ such that

$$
B\left(\mathfrak{M}_{0, S}\right) \cong B_{v, \delta}\left(\mathfrak{M}_{0, S}\right) \otimes_{\mathbb{Q}} \mathbb{Q}\left[\omega_{i_{1} j_{1}}\right] \otimes_{\mathbb{Q}} \cdots \otimes_{\mathbb{Q}} \mathbb{Q}\left[\omega_{i_{\ell} j_{\ell}}\right],
$$

where $\left\{i_{1}, j_{1}\right\}, \ldots,\left\{i_{\ell}, j_{\ell}\right\} \in F_{v}$ are the chords occurring in the triangulation corresponding to $v$. As above, $B_{v, \delta}\left(\mathfrak{M}_{0, S}\right)$ corresponds to the set of all integrable words which do not terminate in any symbol $\omega_{i_{k} j_{k}}$. The case $B_{0}\left(\mathfrak{M}_{0, S}\right)$ corresponds to the vertex whose triangulation is $\{\{2,4\}, \ldots,\{2, n\}\}$. This is just the point $x_{1}=\cdots=x_{\ell}=0$ in cubical coordinates.

\subsection{The dihedral connection on $\mathfrak{M}_{0, S}$}

There is a canonical differential equation on $\mathfrak{M}_{0, S}$ whose solutions can be expressed in terms of multiple polylogarithms. Let $\mathbb{Z}\left\langle\delta_{i j}\right\rangle$ denote the free non-commutative Hopf algebra generated by the symbols $\delta_{i j}=\delta_{j i}$, for $\{i, j\} \in \chi_{S, \delta}$, where $\delta_{i j}$ is primitive (see $\S 3.1$ ). It is convenient to set $\delta_{i i}=\delta_{i i+1}=0$ for all indices $i \in \mathbb{Z} / n \mathbb{Z}$. Consider the following formal 1-form on $\mathfrak{M}_{0, S}$ :

$$
\Omega_{S, \delta}=\sum_{\{i, j\} \in \chi_{S, \delta}} \delta_{i j} \frac{d u_{i j}}{u_{i j}}
$$


The form $\Omega_{S, \delta}$ is integrable if and only if $d \Omega_{S, \delta}=\Omega_{S, \delta} \wedge \Omega_{S, \delta}$. Since $\Omega_{S, \delta}$ is closed, this reduces to $\Omega_{S, \delta} \wedge \Omega_{S, \delta}=0$. We define the dihedral infinitesimal braid relations to be the identities:

$$
\left[\delta_{i-1 j}+\delta_{i j-1}-\delta_{i-1 j-1}-\delta_{i j}, \delta_{k-1 l}+\delta_{k l-1}-\delta_{k-1 l-1}-\delta_{k l}\right]=0,
$$

for all $i, j, k, l \in S$.

For each $1 \leq i, j \leq n$, consider a set of formal symbols $t_{i j}$, where $t_{i i}=0$ and $t_{i j}=t_{j i}$. The Knizhnik-Zamolodchikov (KZ) form on $\left(\mathbb{P}_{*}^{1}\right)^{n}$ is the 1-form:

$$
\Omega_{K Z_{n}}=\sum_{1 \leq i<j \leq n} t_{i j} \Delta_{i j}
$$

where $\Delta_{i j}=\Delta_{j i}$ is given by (6.2). Let us assume that

$$
\sum_{k=1}^{n} t_{k l}=0 \quad \text { for all } 1 \leq l \leq n
$$

This variant of the KZ-equation has been considered by Ihara, amongst others. It corresponds to the usual KZ-equation on $\mathbb{C}_{*}^{n-1}$, except that it has an extra set of symbols at infinity, and one extra relation which kills the center of the braid algebra. One can prove that $\Omega_{K Z_{n}}$ is integrable if and only if the following single relation holds:

$$
\left[t_{i j}, t_{k l}\right]=0 \quad \text { for all } i, j, k, l \text { distinct. }
$$

One verifies by computing $\left[t_{i j}, \sum_{l=1}^{n} t_{k l}\right]=0$, that this is equivalent to the usual infinitesimal braid relations:

$$
\begin{aligned}
{\left[t_{i j}, t_{k l}\right] } & =0, \\
{\left[t_{i j}, t_{i k}+t_{j k}\right] } & =0,
\end{aligned}
$$

which hold for all distinct indices $2 \leq i, j, k, l \leq n$. Now, by (2.6), we have $\omega_{i j}=d \log u_{i j}=$ $\Delta_{i j+1}+\Delta_{i+1 j}-\Delta_{i+1 j+1}-\Delta_{i j}$, for $\{i, j\} \in \chi_{S, \delta}$. If we write

$$
\Omega_{K Z_{n}}=\Omega_{S, \delta}
$$

then this is equivalent to the identities

$$
t_{i j}=\delta_{i j-1}+\delta_{i-1 j}-\delta_{i-1 j-1}-\delta_{i j}
$$

for all $1 \leq i, j \leq n$, as is easily verified. Since $\delta_{i i}=\delta_{i+1}=0$ for $1 \leq i \leq n$, then (6.14) implies that

$$
\begin{aligned}
& t_{i j}=\delta_{i-1 j} \quad \text { if } j=i+1, \\
& t_{i j}=\delta_{i-1 j}-\delta_{i-1 j-1}-\delta_{i j} \quad \text { if } \quad j=i+2 .
\end{aligned}
$$

The following lemma implies that the set of equations (6.14) are invertible over $\mathbb{Z}$.

Lemma 6.7. - For all $1 \leq i<j \leq n$,

$$
\delta_{i j}=\sum_{i<a<b \leq j} t_{a b}
$$


Proof. - By Equation (6.15), $\delta_{i-1 i+1}=t_{i+1}$ for $1<i<n$. Substituting into (6.16) gives $\delta_{i-1 i+2}=t_{i i+2}+t_{i+1}+t_{i+1 i+2}$. Let $m \geq 4$, and suppose by induction that (6.17) holds for all $0<j-i \leq m-1$. Then for $j-i=m-1$, (6.14) gives

$$
\begin{aligned}
\delta_{i-1 j} & =t_{i j}+\delta_{i j}+\delta_{i-1 j-1}-\delta_{i j-1}, \\
& =t_{i j}+\sum_{i<a<b \leq j} t_{a b}+\sum_{i-1<a<b \leq j-1} t_{a b}-\sum_{i<a<b \leq j-1} t_{a b}, \\
& =t_{i j}+\sum_{i<a<b \leq j} t_{a b}+\sum_{i<b \leq j-1} t_{i b}=\sum_{i-1<a<b \leq j} t_{a b} .
\end{aligned}
$$

This proves (6.17) when $j-i=m$. The result follows by induction.

Now if we substitute the expressions (6.14) for $t_{i j}$ and $t_{k l}$ in terms of $\delta_{a b}$ in Equation (6.12), then we obtain (6.9). This proves the following result.

Proposition 6.8. - The form $\Omega_{S, \delta}$ is integrable if and only if the dihedral braid relations (6.9) hold.

Lemma 6.9. - The dihedral braid relations imply that

$$
\left[\delta_{i j}, \delta_{k l}\right]=0
$$

for all chords $\{i, j\},\{k, l\} \in \chi_{S, \delta}$ which do not cross.

Proof. - Without loss of generality, we can assume that $1 \leq i<j<k<l \leq n$. Then, by identity (6.12),

$$
\left[\delta_{i j}, \delta_{k l}\right]=\left[\sum_{i<a<b \leq j} t_{a b}, \sum_{k<c<d \leq l} t_{c d}\right]=0
$$

since all sets of four indices $\{a, b, c, d\}$ occurring in the summation are distinct.

Example 6.10. - In the case $S=\{1,2,3,4,5\}$, relation (6.9) with $i=2, j=4, k=3$, $l=5$ implies that $\left[\delta_{14}-\delta_{13}-\delta_{24}, \delta_{25}-\delta_{35}-\delta_{24},\right]=0$. By (6.18) this gives the following five-term relation:

$$
\left[\delta_{13}, \delta_{24}\right]+\left[\delta_{24}, \delta_{35}\right]+\left[\delta_{35}, \delta_{41}\right]+\left[\delta_{41}, \delta_{52}\right]+\left[\delta_{52}, \delta_{13}\right]=0
$$

This is dual to the functional equation of the dilogarithm.

Definition 6.11. - Let $R$ denote a commutative unitary ring, and let $I$ denote the ideal in $R\left\langle\delta_{i j}:\{i, j\} \in \chi_{S, \delta}\right\rangle$ generated by the dihedral relations (6.9) above. The dihedral braid algebra over $R$ is the free non-commutative $R$-algebra

$$
\mathfrak{B}_{S, \delta}(R)=R\left\langle\delta_{i j}:\{i, j\} \in \chi_{S, \delta}\right\rangle / I \text {. }
$$

This is a co-commutative graded Hopf algebra over $R(\S 3.1)$, where $\operatorname{deg} \delta_{i j}=1$. The product is the concatenation product, and the coproduct $\Gamma$ is the unique coproduct with respect to which the generators $\delta_{i j}$ are primitive ( $I$ is a Hopf ideal because it is generated by commutators of primitive elements). It is the universal enveloping algebra of the free Lie algebra generated by the symbols $\delta_{i j}$, subject to relation (6.9). As in $\S 3.1$, its completion is the $R$-Hopf algebra

$$
\widehat{\mathfrak{B}}_{S, \delta}(R)=R\left\langle\left\langle\delta_{i j}:\{i, j\} \in \chi_{S, \delta},\right\rangle\right\rangle / \widehat{I}
$$


where $\widehat{I}$ is the closed ideal generated by $I$. It follows from the previous calculations that $\mathfrak{B}_{S, \delta}(R)$ is just the free non-commutative $R$-algebra generated by the symbols $t_{i j}$, for $1 \leq i, j \leq n$, which satisfy (6.11), modulo the relations (6.12). This is isomorphic to the ordinary infinitesimal braid algebra modulo its center. The difference here is that we have fixed a set of generators for this algebra which depend on the dihedral structure $\delta$.

Let $\widehat{\mathfrak{M}}_{0, S}$ be a universal covering space for $\mathfrak{M}_{0, S}$, and let $p: \widehat{\mathfrak{M}}_{0, S} \rightarrow \mathfrak{M}_{0, S}$ denote the projection map. A multi-valued function on $\mathfrak{M}_{0, S}$ is defined to be a holomorphic function on $\widehat{\mathfrak{M}}_{0, S}$. Since the integrability conditions are satisfied in $\mathfrak{B}_{S, \delta}(\mathbb{C})$ we can consider the following formal differential equation on $\widehat{\mathfrak{M}}_{0, S}$ :

$$
d L=\Omega_{S, \delta} L .
$$

A solution $L$ takes values in $\widehat{\mathfrak{B}}_{S, \delta}(\mathbb{C})$. Its coefficients are multi-valued functions on $\mathfrak{M}_{0, S}$. We can fix a solution to (6.22) by specifying its value at a point of $\mathfrak{M}_{0, S}$, or its limiting value at an intersection of boundary divisors. It suffices to define solutions at intersections of divisors of maximal codimension. Therefore, we define

$$
V^{\delta}=\left\{\alpha \in \chi_{S, \delta}^{\ell}\right\}
$$

to be the set of all triangulations of the $n$-gon. By $\S 2.7$, each such triangulation determines a unique vertex of the associahedron $\bar{X}_{S, \delta}$. For each vertex $v \in V^{\delta}$, let $F_{v}=\left\{\{i, j\} \in \chi_{S, \delta}\right.$ : $\left.u_{i j}(v)=0\right\}$ denote the set of faces of the associahedron $\bar{X}_{S, \delta}$ which meet at $v$. Let $\log \left(u_{i j}\right)$, for $\{i, j\} \in \chi_{S, \delta}$, denote the principal branch of the logarithm on $u_{i j}>0$ (see $\S 4$.2).

Theorem 6.12. - Let $v \in V^{\delta}$. There exists a unique solution $L_{v, \delta}$ to (6.22) such that in a neighbourhood of $v$,

$$
L_{v, \delta}(\underline{u})=f_{v, \delta}(\underline{u}) \prod_{\{i, j\} \in F_{v}} \exp \left(\delta_{i j} \log \left(u_{i j}\right)\right),
$$

where $f_{v, \delta}(\underline{u}) \in \widehat{\mathfrak{B}}_{S, \delta}(\mathbb{C})$ extends to a holomorphic function in the neighbourhood of $v \in \mathfrak{M}_{0, S}^{\delta}$, and takes the value 1 at $v$. The function $f_{v, \delta}(\underline{u})$ extends holomorphically to an open neighbourhood of the interior of every face $F$ meeting $v$.

REMARK 6.13. - The product $\prod_{\{i, j\} \in F_{v}} \exp \left(\delta_{i j} \log \left(u_{i j}\right)\right)$ is well-defined, because by (6.18), the symbols $\delta_{i j}$ and $\delta_{k l}$ commute whenever $\{i, j\}$ and $\{k, l\}$ do not cross, and no two chords $\{i, j\},\{k, l\} \in F_{v}$ can cross because $F_{v}$ is a triangulation of the $n$-gon $(S, \delta)$.

Proof. - Let $\mathfrak{B}_{S, \delta}^{>0}(\mathbb{C}) \subset \mathfrak{B}_{S, \delta}(\mathbb{C})$ denote the kernel of the counit $\varepsilon: \mathfrak{B}_{S, \delta}(\mathbb{C}) \rightarrow \mathbb{C}$. For each integer $N \geq 1$, define

$$
W_{N}=\mathfrak{B}_{S, \delta}(\mathbb{C}) /\left(\mathfrak{B}_{S, \delta}^{>0}(\mathbb{C})\right)^{N+1} .
$$

If we write $\delta_{i j}$ for the map which acts by left multiplication by the symbol $\delta_{i j}$, for each $\{i, j\} \in \chi_{S, \delta}$, then each $\delta_{i j}$ is a nilpotent operator on the space $W_{N}$.

In $\S 4.1$ we showed that $\bar{X}_{S, \delta}$ is a manifold with corners by constructing a specific atlas $\left\{U_{e}(\varepsilon)\right\}$. We will show that $\Omega_{S, \delta}$ defines a unipotent equation of Fuchs' type on each chart (Definition 4.7), and apply the results of $\S 4.3$. Therefore, let $\alpha \in \chi_{S, \delta}^{k}$ denote a partial decomposition of the $n$-gon, where $1 \leq k \leq \ell$. To $\alpha$ corresponds the face $F_{\alpha}$ of $\bar{X}_{S, \delta}$. Choose 
any complete triangulation $\alpha^{\prime} \in \chi_{S, \delta}^{\ell}$ which contains $\alpha$. By Proposition (2.22), the vertex coordinates

$$
\left\{x_{i}^{\alpha^{\prime}}: 1 \leq i \leq \ell\right\}=\left\{u_{i j}:\{i, j\} \in \alpha^{\prime}\right\}
$$

form a system of normal coordinates in a neighbourhood of $F_{\alpha}$. We can therefore write

$$
\Omega_{S, \delta}=\sum_{\{i, j\} \in \alpha^{\prime}} \delta_{i j} \frac{d u_{i j}}{u_{i j}}+A_{i j} d u_{i j},
$$

where $A_{i j}$ are holomorphic functions in a neighbourhood of $F_{\alpha}$. Since the operators $\delta_{i j}$ are nilpotent on $W_{N}$, and since the open neighbourhoods of every face $F_{\alpha}$ (including $F_{\varnothing}=X_{S, \delta}$ ) cover $\bar{X}_{S, \delta}$, it follows that (6.22) is unipotent of Fuchs' type, as required.

By Theorem 4.6, we can find a local solution $L_{v, \delta}^{(N)}$ to $(6.22)$ with values in $W_{N}(\mathbb{C})$, which satisfies the asymptotic condition stated above. By Corollary 4.8 , this solution extends globally over the whole Stasheff polytope $\bar{X}_{S, \delta}$. The theorem follows on taking the limit as $N$ tends to infinity, since $\widehat{\mathfrak{B}}_{S, \delta}(\mathbb{C})=\lim _{\leftarrow} \mathfrak{B}_{S, \delta}(\mathbb{C}) /\left(\mathfrak{B}_{S, \delta}^{>0}(\mathbb{C})\right)^{N}$.

Any such solution $L_{v, \delta}$ to (6.22) extends by analytic continuation to give a multi-valued function on the whole of $\mathfrak{M}_{0, S}$. By construction, the theorem defines a unique real-valued branch on the interior of the associahedron $X_{S, \delta}$. It is convenient to write the asymptotic boundary condition

$$
L_{v, \delta} \sim \prod_{\{i, j\} \in F_{v}} u_{i j}^{\delta_{i j}} \text { near } v
$$

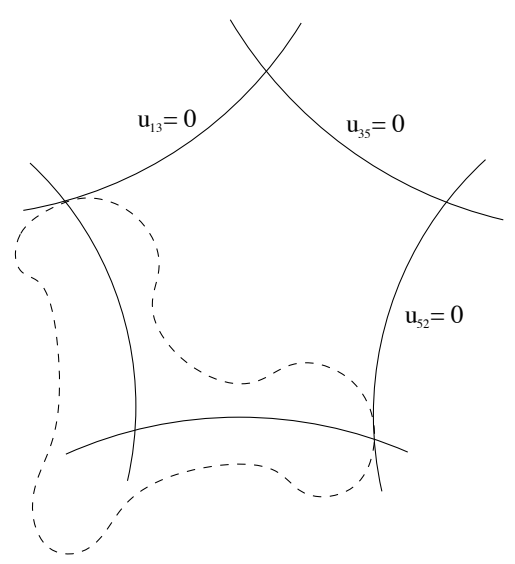

Figure 14. A local picture of $\overline{\mathfrak{M}}_{0,5}(\mathbb{R})$ and a cell $X_{5, \delta_{i}}$. The dashed region depicts the set of real points of a domain of holomorphy for the regularised function $f_{v, \delta}$ given in Theorem 6.12 .

REMARK 6.14. - The formal equation (6.22) is a homogeneous version of the KnizhnikZamolodchikov equation on $\mathbb{C}_{*}^{n-1}$. Drinfeld studied solutions to the $\mathrm{KZ}$ equation on $\mathbb{C}_{*}^{3}, \mathbb{C}_{*}^{4}$ with prescribed asymptotics in certain zones [18], which were subsequently generalised by Kapranov [36]. Such a zone is determined by a permutation on $n-1$ letters, plus a bracketing on the set with $n-1$ letters. Combinatorially, a permutation on $n-1$ letters corresponds to 
a cyclic structure on $n$ letters, and a bracketing corresponds to a triangulation of an $n$-gon, i.e., a vertex in the associahedron of dimension $n-3$ (fig. 11). Kapranov interpreted each zone as the region near a corner at infinity in a certain two-fold cover $\widetilde{S}$ of the compactified real moduli space:

$$
\widetilde{S} \rightarrow \overline{\mathfrak{M}}_{0, S}(\mathbb{R}) .
$$

In the previous theorem, we have constructed a canonical solution $L_{v, \delta}$ near each corner on $\overline{\mathfrak{M}}_{0, S}(\mathbb{R})$. Therefore, each solution $L_{v, \delta}$ corresponds to exactly two Drinfeld-Kapranov zones. One way to see this is that the manifold $\widetilde{S}$ is obtained by gluing together a set of associahedra which are parametrized by the set of all cyclic, rather than dihedral structures. The number of cyclic structures on $S$, where $|S|=n$ is exactly $(n-1)$ !. As an example, let us consider the simplest case $\mathfrak{M}_{0,4}$. Then Equation (6.22) reads:

$$
\frac{d L}{d x}=\left(\frac{\delta_{13}}{x}+\frac{\delta_{24}}{x-1}\right) L .
$$

Consider the solution $L_{0^{+}}(x)$ which satisfies $L_{0^{+}}(x) \sim \exp \left(\delta_{13} \log x\right)$ as $x \rightarrow 0^{+}$. Remembering that $x=u_{13}=[12 \mid 43]$, and after placing the point $z_{4}$ at $\infty$, we obtain $x=\left(z_{2}-z_{3}\right) /\left(z_{1}-z_{3}\right)$. If $0<x<1$, then either $z_{1}<z_{2}<z_{3}$ or $z_{3}<z_{2}<z_{1}$. These are the two cyclic structures on $\left\{z_{1}, z_{2}, z_{3}, \infty\right\}$ which map to the dihedral structure $\delta$ corresponding to the cell $[0,1]=\bar{X}_{S, \delta}$. The zone $0<x \ll 1$ therefore corresponds to the pair of zones $z_{1}\left(z_{2} z_{3}\right)$ and $\left(z_{3} z_{2}\right) z_{1}$ in Kapranov's notations.

\subsection{Functoriality with respect to projection maps}

It is well-known that solutions to the KZ-equation decompose as products of hyperlogarithm equations by considering fibration maps between configuration spaces. One obtains a more general decomposition result for the homogeneous equation (6.22), by using the projection maps considered in $\S 2.4$. First, we fix a dihedral structure $\delta$ on $S$ and choose a chord $\{i, j\} \in \chi_{S, \delta}$. Recall from $\S 2.4$, that if we set $T_{1}=\left\{s_{j+1}, \ldots, s_{i}, s_{i+1}\right\}$, and $T_{2}=\left\{s_{i}, s_{i+1}, \ldots, s_{j}\right\}$, and denote the induced dihedral structures by $\delta_{1}, \delta_{2}$, then $T_{1} \cap T_{2}=\left\{s_{i}, s_{i+1}\right\}$, and there is a projection map:

$$
f_{T_{1}} \times f_{T_{2}}: \mathfrak{M}_{0, S}^{\delta} \longrightarrow \mathfrak{M}_{0, T_{1}}^{\delta_{1}} \times \mathfrak{M}_{0, T_{2}}^{\delta_{2}} .
$$

By Lemma 2.6, this map has a section whose image is the divisor $D_{i j}=\left\{u_{i j}=0\right\}$ :

$$
i: \mathfrak{M}_{0, T_{1}}^{\delta_{1}} \times \mathfrak{M}_{0, T_{2}}^{\delta_{2}} \stackrel{\sim}{\longrightarrow} D_{i j} \subset \mathfrak{M}_{0, S}^{\delta} .
$$

In order to fix solutions of (6.22), let $v \in V^{\delta}$ be a vertex of the polytope $\bar{X}_{S, \delta}$ such that $v \in D_{i j}$, i.e., $u_{i j}(v)=0$. By projecting down, we obtain vertices in $\bar{X}_{T_{k}, \delta_{k}}$ :

$$
v_{k}=f_{T_{k}}(v) \in \bar{X}_{T_{k}, \delta_{k}} \quad \text { for } k=1,2 .
$$

If $v$ is given by a triangulation $\alpha \in \chi_{S, \delta}^{\ell}$ of the $n$-gon $S$, then $v_{1}, v_{2}$ are given by the restrictions $\alpha_{1}, \alpha_{2}$ of this triangulation to $T_{1}$ and $T_{2}$ respectively (compare fig. 4). As sets of chords, we have $\alpha=\alpha_{1} \sqcup \alpha_{2} \sqcup\{i, j\}$. We need an extra technical condition that the only chord in $\alpha$ emanating from the vertex $(j)$ is the chord $\{i, j\}$ (the dihedral coordinate corresponding to such a chord would not be preserved since $f_{T_{1}}$ and $f_{T_{2}}$ contract one of the edges $j$ or $j+1$ ). Let

$$
i_{T_{1}}: \mathfrak{M}_{0, T_{1}}^{\delta_{1}} \longrightarrow \mathfrak{M}_{0, T_{1}}^{\delta_{1}} \times \mathfrak{M}_{0, T_{2}}^{\delta_{2}} \longrightarrow D_{i j}
$$


denote the map which sends $x$ to $i\left(x, v_{2}\right) \in D_{i j}$. Define a map $i_{T_{2}}$ similarly, and set

$$
\pi_{k}=i_{T_{k}} \circ f_{T_{k}} \quad \text { for } k=1,2 .
$$

Then $\pi_{k}$ is a projection map

$$
\pi_{k}: \mathfrak{M}_{0, S}^{\delta} \rightarrow D_{\alpha_{k} \sqcup\{i, j\}} \quad \text { for } k=1,2,
$$

because $f_{T_{k}} \circ i_{T_{k}}$ is the identity. We can write these maps explicitly in vertex coordinates $x_{1}^{\alpha}, \ldots, x_{\ell}^{\alpha}$, which form a local system of coordinates on $\mathfrak{M}_{0, S}^{\delta}(\mathbb{C})$ (see $\left.\S 2.6\right)$. We can choose an ordering on $\alpha$ such that $D_{\alpha_{2}}=\left\{x_{1}^{\alpha}=\cdots=x_{m-1}^{\alpha}=0\right\}, u_{i j}=x_{m}^{\alpha}$, and $D_{\alpha_{1}}=\left\{x_{m+1}^{\alpha}=\cdots=x_{\ell}^{\alpha}=0\right\}$ for some $m$. In that case, we have

$$
f_{T_{1}} \times f_{T_{2}}:\left(x_{1}^{\alpha}, \ldots, x_{\ell}^{\alpha}\right) \longrightarrow\left(\left(x_{1}^{\alpha}, \ldots, x_{m-1}^{\alpha}\right),\left(x_{m+1}^{\alpha}, \ldots, x_{\ell}^{\alpha}\right)\right),
$$

and

$$
\begin{aligned}
& \pi_{1}:\left(x_{1}^{\alpha}, \ldots, x_{\ell}^{\alpha}\right) \longmapsto\left(x_{1}^{\alpha}, \ldots, x_{m-1}^{\alpha}, 0, \ldots, 0\right) \\
& \pi_{2}:\left(x_{1}^{\alpha}, \ldots, x_{\ell}^{\alpha}\right) \longmapsto\left(0, \ldots, 0, x_{m+1}^{\alpha}, \ldots, x_{\ell}^{\alpha}\right) .
\end{aligned}
$$

We shall write $\partial / \partial u_{i j}$ to denote partial differentiation with respect to the vertex coordinate $x_{m}^{\alpha}=u_{i j}$ in a neighbourhood of $v \in \mathfrak{M}_{0, S}^{\delta}(\mathbb{C})$.

Let $L_{v, \delta}$ denote the unique solution to (6.22) on $\mathfrak{M}_{0, S}$ given by Theorem 6.12 . We define

$$
L_{k}=L \circ \pi_{k}, \quad \text { for } k=1,2 .
$$

Then the functions $L_{k}: \widehat{\mathfrak{M}}_{0, S} \rightarrow \widehat{\mathfrak{B}}_{S, \delta}(\mathbb{C})$ satisfy the differential equation

$$
d L_{k}=\Omega_{k} L_{k}, \quad \text { for } k=1,2,
$$

where $\Omega_{k}$ is given by:

$$
\Omega_{k}=\left(\pi_{k}\right)_{*} \Omega_{S, \delta}=\sum_{\{a, b\} \in \chi_{T_{k}, \delta_{k}}} \delta_{a b} \frac{d u_{a b}}{u_{a b}}, \quad \text { for } k=1,2 .
$$

By construction, the solutions $L_{k}$ satisfy the asymptotic condition

$$
L_{k}=f_{k}\left(\sum_{\{a, b\} \in \alpha_{k}} \delta_{a b} \log u_{a b}\right), \quad \text { for } k=1,2,
$$

where $f_{k}$ is holomorphic in a neighbourhood of $v_{k}$ on $D_{\alpha_{k}}$, where it takes the value 1 . It is clear that $\Omega_{k}$ and $L_{k}$ only involve the symbols $\delta_{a b}$ where $\{a, b\} \in \chi_{T_{k}, \delta_{k}}$. Since no chord in $\chi_{T_{1}, \delta_{1}}$ crosses any chord in $\chi_{T_{2}, \delta_{2}}$, it follows from (6.18) that $L_{1}$ and $L_{2}$ commute. Likewise, we have $\left[L_{1}, \Omega_{2}\right]=\left[L_{2}, \Omega_{1}\right]=\left[\Omega_{1}, \Omega_{2}\right]=0$. The three series $L_{v, \delta}, L_{1}, L_{2}$ are all formal power series in $\widehat{B}_{S, \delta}(\mathbb{C})$ whose coefficients are multi-valued functions on $\mathfrak{M}_{0, S}$. They are related as follows.

Proposition 6.15. - Let $\{i, j\} \in \chi_{S, \delta}$ be any chord, and let $v \in V^{\delta}$ such that $u_{i j}(v)=0$. With the notations above, there is a decomposition

$$
L_{v, \delta}=h L_{1} L_{2}
$$

where $h$ is the unique solution in $\widehat{B}_{S, \delta}(\mathbb{C})$ to the hyperlogarithm quotient equation

$$
\frac{\partial h}{\partial u_{i j}}=\left(\sum_{\{k, l\} \in \chi_{S, \delta}} \delta_{k l} \frac{\partial \log u_{k l}}{\partial u_{i j}}\right) h,
$$


which satisfies the boundary condition

$$
h=g \exp \left(\delta_{i j} \log u_{i j}\right)
$$

where $g$ is a holomorphic function of $u_{i j}$ in a neighbourhood of 0 , and $\left.g\right|_{u_{i j}=0}$ is the constant function 1.

Proof. - Define a formal power series $h \in \widehat{B}_{S, \delta}(\mathbb{C})$ by the equation $h=L_{v, \delta} L_{2}^{-1} L_{1}^{-1}$. If we differentiate this equation, we deduce that

$$
\Omega_{S, \delta} L_{v, \delta}=d h L_{1} L_{2}+h \Omega_{1} L_{1} L_{2}+h \Omega_{2} L_{1} L_{2},
$$

where we have used the fact that $\left[\Omega_{2}, L_{1}\right]=0$. It follows that

$$
d h=\Omega_{S, \delta} h-h\left(\Omega_{1}+\Omega_{2}\right) .
$$

By definition, the functions $L_{k}$ do not depend on the variable $u_{i j}$, i.e., $\partial L_{k} / \partial u_{i j}=0$, for $k=1,2$. Therefore $\partial h / \partial u_{i j}=\partial L_{v, \delta} / \partial u_{i j} L_{2}^{-1} L_{1}^{-1}$, and (6.22) implies that

$$
\frac{\partial h}{\partial u_{i j}}=\left(\sum_{\{k, l\} \in \chi_{S, \delta}} \frac{\delta_{k l}}{u_{k l}} \frac{\partial u_{k l}}{\partial u_{i j}}\right) h .
$$

By definition of the solution $L_{v, \delta}$ and Equations (6.26), we have

$h=f_{v, \delta} \exp \left(\sum_{\{a, b\} \in \alpha} \delta_{a b} \log u_{a b}\right) \exp \left(-\sum_{\{a, b\} \in \alpha_{2}} \delta_{a b} \log u_{a b}\right) f_{2}^{-1} \exp \left(-\sum_{\{a, b\} \in \alpha_{1}} \delta_{a b} \log u_{a b}\right) f_{1}^{-1}$,

where $f_{v, \delta}$ is holomorphic in a neighbourhood of $v$, and $\alpha_{1}, \alpha_{2}, \alpha$ are the triangulations of $T_{1}, T_{2}, S$ corresponding to $v_{1}, v_{2}, v$ respectively. Since $L_{1}$ and $L_{2}$ commute,

$$
h=f_{v, \delta} \exp \left(\delta_{i j} \log u_{i j}\right) f_{2}^{-1} f_{1}^{-1}=f_{v, \delta} f_{2}^{-1} f_{1}^{-1} \exp \left(\delta_{i j} \log u_{i j}\right) .
$$

Let $g=f_{v, \delta} f_{2}^{-1} f_{1}^{-1}=f_{v, \delta} f_{1}^{-1} f_{2}^{-1}$, which is holomorphic in the neighbourhood of $v$. In order to complete the proof, it suffices to show that the function $\left.g\right|_{u_{i j}=0}$ is the constant function 1 . This, along with the differential equation for $h$, will determine $h$ uniquely. Let $G$ denote the restriction of $g$ to the divisor $D_{i j}=\left\{u_{i j}=0\right\}$. We already know by construction that $G(v)=1$. Since $g$ is holomorphic in the neighbourhood of $u_{i j}=0, G$ satisfies a differential equation which is obtained by projecting (6.30) onto $u_{i j}=0$, which amounts to pulling back $\Omega_{S, \delta}$ by $\left(\pi_{1} \times \pi_{2}\right)_{*}$. By definition,

$$
\left.\Omega_{S, \delta}\right|_{D_{i j}}=\Omega_{1}+\Omega_{2}
$$

Equation (6.30) therefore restricts to give the following differential equation for $G$ :

$$
d G=\left[\Omega_{1}+\Omega_{2}, G\right],
$$

where $G(v)=1$. This equation only has constant solutions. To see this, consider the conjugate $H=\left(L_{1} L_{2}\right)^{-1} G L_{1} L_{2}$. Substituting into (6.31) gives $d H=0$. Therefore $H$ is the constant function 1 , and so the same is true of $G$, which completes the proof.

One can verify from the definitions that the map $f_{T_{k}}$ induces a map

$$
\left(f_{T_{k}}\right)_{*}: \mathfrak{B}_{S, \delta}(\mathbb{C}) \longrightarrow \mathfrak{B}_{T_{k}, \delta_{k}}(\mathbb{C}), \quad \text { for } k=1,2,
$$


which sends $\delta_{a b}$ to zero for all chords $\{a, b\}$ which are not in $\chi_{T_{k}, \delta_{k}}$, and is the identity on $\delta_{a b}$ for all chords $\{a, b\} \in \chi_{T_{k}, \delta_{k}}$. This also follows immediately from the fact that $\Omega_{k}=\left(\pi_{k}\right)_{*} \Omega_{S, \delta}$ is integrable. We can then consider

$$
\left(f_{T_{k}}\right)_{*} L_{k}: \mathfrak{M}_{0, T_{k}} \longrightarrow \widehat{\mathfrak{B}}_{T_{k}, \delta_{k}}(\mathbb{C}) \quad \text { for } k=1,2 .
$$

By (6.26) and the uniqueness part of Theorem 6.12, we conclude that

$$
L_{v_{k}, \delta_{k}}=\left(f_{T_{k}}\right)_{*} L_{k} \quad \text { for } k=1,2 .
$$

In conclusion, a solution $L_{v, \delta}$ to (6.22) on $\mathfrak{M}_{0, S}$ is equivalent to a pair of solutions $L_{v_{k}, \delta_{k}}$ on $\mathfrak{M}_{0, T_{k}}$ for $k=1,2$ plus a solution to the hyperlogarithm quotient equation (6.28). Note that many of the terms in the differential equation (6.28) vanish.

Example 6.16. - Consider the case $\mathfrak{M}_{0,6}$, and let $\{i, j\}=\{2,5\}$ (see fig. 3 ). Then $T_{1}=\{2,3,4,5\}$ and $T_{2}=\{6,1,2,3\}$. We shall work in cubical coordinates, and write $\left(x_{1}, x_{2}, x_{3}\right)=(x, y, z)$. Then $u_{24}=x, u_{25}=y$, and $u_{26}=z$. The map $\mathfrak{M}_{0,6} \rightarrow D_{25} \cong \mathfrak{M}_{0, T_{1}} \times \mathfrak{M}_{0, T_{2}}$ is given by projecting onto the divisor $y=0$. Therefore $L_{1}(x, y, z)=L(x, 0,0)$, and $L_{2}(x, y, z)=L(0,0, z)$ and we have:

$$
\begin{aligned}
& d L_{1}=\left(\delta_{24} \frac{d x}{x}+\delta_{35} \frac{d x}{x-1}\right) L_{1}, \\
& d L_{2}=\left(\delta_{26} \frac{d z}{z}+\delta_{15} \frac{d z}{z-1}\right) L_{2} .
\end{aligned}
$$

Thus $L_{1}, L_{2}$ are generating series of multiple polylogarithms in one variable (but note that there is a difference in sign in [7]). On the other hand, $h$ is the unique solution to

$$
\begin{aligned}
\frac{\partial h}{\partial y} & =\left(\frac{\delta_{25}}{y}+\frac{t_{56}}{y-1}+\frac{t_{46}}{y-x^{-1}}+\frac{t_{15}}{y-z^{-1}}+\frac{t_{14}}{y-(x z)^{-1}}\right) h, \\
h & \sim \exp \left(\delta_{25} \log y\right) \quad \text { as } y \rightarrow 0,
\end{aligned}
$$

where, according to (6.14), $t_{56}=\delta_{46}, t_{15}=\delta_{14}-\delta_{15}-\delta_{46}, t_{46}=\delta_{36}-\delta_{46}-\delta_{35}$, and $t_{14}=\delta_{13}+\delta_{46}-\delta_{14}-\delta_{36}$. By (6.13), there are commutation relations

$$
\left[t_{14}, t_{56}\right]=0, \quad \text { and } \quad\left[t_{15}, t_{46}\right]=0 .
$$

Therefore (6.32) is a hyperlogarithm quotient equation on the punctured affine line $\mathbb{P}^{1} \backslash\left\{0,1, \infty, x^{-1}, z^{-1},(x z)^{-1}\right\}$. Compare Remark 2.11.

By applying the proposition repeatedly, we obtain an explicit decomposition of the generating series $L_{v, \delta}$ as products of hyperlogarithms. Let us apply the proposition in the case where $\{i, j\}=\{2, n\}$. In cubical coordinates, $u_{2 n}=x_{\ell}$, and one can check that $h$ is the unique function satisfying

$$
\begin{aligned}
\frac{\partial h}{\partial x_{\ell}} & =\left(\frac{\delta_{2 n}}{x_{\ell}}+\frac{\delta_{1 n-1}}{x_{\ell}-1}+\sum_{i=1}^{\ell-1} \frac{\delta_{i+3 n}+\delta_{i+21}-\delta_{i+2 n}-\delta_{i+31}}{x_{\ell}-\left(x_{i} \ldots x_{\ell-1}\right)^{-1}}\right) h, \\
h & \sim \exp \left(\delta_{2 n} \log x_{\ell}\right) \quad \text { as } \quad x_{\ell} \rightarrow 0,
\end{aligned}
$$

where, as usual, $\delta_{i i}=\delta_{i-1 i}=0$ by convention. The function $\log x$ is the unique branch satisfying $\log 1=0$. This defines a multi-valued function on $\mathbb{P}^{1} \backslash \Sigma$ where $\Sigma=\left\{\sigma_{0}, \ldots, \sigma_{\ell}\right\}$, with

$$
\sigma_{0}=0, \quad \sigma_{1}=1, \quad \sigma_{2}=x_{\ell-1}^{-1}, \quad \ldots, \quad \sigma_{\ell-1}=\left(x_{1} \ldots x_{\ell-1}\right)^{-1} .
$$


The series $h \exp \left(-\delta_{2 n} \log x_{\ell}\right)$ is holomorphic in the neighbourhood of $x_{\ell}=0$. In this case, the projection map $f_{T_{1}} \times f_{T_{2}}$ is a fibration. It follows that $h$ is a hyperlogarithm equation (i.e., there are no relations between the coefficients in (6.33)). Notice also that the coefficient $\delta_{i+3 n}+\delta_{i+21}-\delta_{i+2 n}-\delta_{i+31}$ is just $t_{1 i+3}$.

By substituting the above values of $\sigma_{i}$ into the formula (5.3), we deduce that the coefficients of the formal power series $h$ are the multiple polylogarithms (§5.4):

$$
\operatorname{Li}_{n_{1}, \ldots, n_{r}}\left(\frac{x_{j_{1}} \ldots x_{\ell}}{x_{j_{2}} \ldots x_{\ell}}, \ldots, \frac{x_{j_{r-1}} \ldots x_{\ell}}{x_{j_{r}} \ldots x_{\ell}}, x_{j_{r}} \ldots x_{\ell}\right),
$$

where $1 \leq j_{1}, \ldots, j_{r} \leq \ell$ are any indices. By applying the proposition inductively, we obtain an explicit decomposition of $L_{v, \delta}$ in terms of hyperlogarithm generating series $h$.

Corollary 6.17. $-L_{v, \delta}$ is a product of hyperlogarithm generating series. Its coeffcients are sums of products of multiple polylogarithms of the form (6.34) with the functions $\log x_{1}, \ldots, \log x_{\ell}$.

\subsection{Regularised zeta series and monodromy}

The monodromy of the KZ equation was first computed by Drinfeld [18]. We shall follow the argument given in [34] for $\mathfrak{M}_{0,4} \cong \mathbb{P}^{1} \backslash\{0,1, \infty\}$ (see also [28]). Consider two vertices $u, v \in V^{\delta}$. By Theorem 6.12, each vertex defines a generating series of multi-valued functions $L_{u, \delta}, L_{v, \delta}$ on $\mathfrak{M}_{0, S}$. The ratio of any two solutions to (6.22) is a constant series.

Definition 6.18. - The regularised zeta series corresponding to $u, v \in V^{\delta}$ is

$$
Z^{u, v}=\left(L_{u, \delta}(x)\right)^{-1} L_{v, \delta}(x) \in \widehat{\mathfrak{B}}_{S, \delta}(\mathbb{C}),
$$

for any $x \in X_{S, \delta}$, i.e., $x=\left(u_{i j}\right)$, where $0<u_{i j}<1$.

Since $L_{v, \delta}$ is real-valued on $X_{S, \delta}, Z^{u, v} \in \widehat{\mathfrak{B}}_{S, \delta}(\mathbb{R})$ has real coefficients. Clearly

$$
Z^{u, v} Z^{v, w}=Z^{u, w}
$$

for all $u, v, w \in V^{\delta}$, and in particular, $Z^{u, v} Z^{v, u}=1$. The zeta series describe the limiting behaviour of a solution of (6.22) near the boundary of $\bar{X}_{S, \delta}$.

Lemma 6.19. - For all $u, v \in V^{\delta}$,

$$
Z^{u, v}=\lim _{x \rightarrow u} \prod_{\{i, j\} \in F_{u}} \exp \left(-\delta_{i j} \log u_{i j}\right) L_{v, \delta}(x),
$$

where $x=\left(u_{i j}\right) \in X_{S, \delta}$.

Proof. - Let $x=\left(u_{i j}\right)$ and let $x \rightarrow u$ along a path in $\bar{X}_{S, \delta}$. By Theorem 6.12,

$$
L_{v, \delta}(x)=L_{u, \delta}(x) Z^{u, v}=f_{u, \delta}\left(\prod_{\{i, j\} \in F_{u}} \exp \left(\delta_{i j} \log u_{i j}\right)\right) Z^{u, v}
$$

which implies that

$$
Z^{u, v}=\lim _{x \rightarrow u}\left(\prod_{\{i, j\} \in F_{u}} \exp \left(-\delta_{i j} \log u_{i j}\right)\right) f_{u, \delta}^{-1} L_{v, \delta}(x) .
$$

But $f_{u, \delta}^{-1}$ is a non-commutative series which is holomorphic in a neighbourhood of $u$ where it takes the value 1 . We can write $f_{u, \delta}^{-1}=1+g(x)$, where $g$ is holomorphic and vanishes at 
$x=u$. Since $z \log ^{n} z \rightarrow 0$ as $z \rightarrow 0$ for all $n \in \mathbb{N}$, and since $L_{v, \delta}(x)$ has at most logarithmic singularities at $x=u$, we deduce that only the constant term 1 in $f_{u, \delta}^{-1}$ gives a non-zero contribution in the limit, which proves the result.

THEOREM 6.20. - The coefficients of the series $Z^{u, v}$ are multiple zeta values:

$$
Z^{u, v} \in \mathcal{Z}\left\langle\left\langle\delta_{i j}:\{i, j\} \in \chi_{S, \delta}\right\rangle\right\rangle / I \quad \text { for all } u, v \in V^{\delta},
$$

where I denotes the closed ideal generated by the dihedral braid relations (6.9).

Proof. - By the relations (6.35), it suffices to compute the coefficients of $Z^{u, v}$, where $u, v$ are adjacent corners of $\bar{X}_{S, \delta}$. In other words, $u, v$ are given by triangulations $\alpha, \beta \in \chi_{S, \delta}^{\ell}$ which differ by one chord only. Let us write $\left\{a, a^{\prime}\right\}=\alpha \backslash \alpha \cap \beta$ and $\left\{b, b^{\prime}\right\}=\beta \backslash \alpha \cap \beta$. Since $D_{\alpha \cap \beta}$ is of dimension 1, there is an isomorphism

$$
i_{u v}: \mathfrak{M}_{0, T}^{\delta^{\prime}} \stackrel{\sim}{\longrightarrow} D_{\alpha \cap \beta} \subset \mathfrak{M}_{0, S}^{\delta},
$$

where $|T|=4$, which maps the cell $\bar{X}_{T, \delta^{\prime}}$ onto the 1-dimensional face of $\bar{X}_{S, \delta}$ which connects $u$ and $v$. Consider the solution $L_{u, \delta}$ to (6.22) given by Theorem 6.12. We can identify $\mathfrak{M}_{0, T}$ with $\mathbb{P}^{1} \backslash\{0,1, \infty\}$ and $X_{T, \delta^{\prime}}$ with the interval $(0,1)$ in such a way that $i_{u v}(0)=u$ and $i_{u v}(1)=v$. The pull-back $F=\left(i_{u v}\right)_{*} L_{u, \delta}$ then satisfies the differential equation:

$$
\begin{aligned}
\frac{d F}{d x} & =\left(\frac{\delta_{u}}{x}+\frac{\delta_{v}}{x-1}\right) F, \\
F & \sim \exp \left(\delta_{u} \log x\right) \quad \text { as } x \rightarrow 0,
\end{aligned}
$$

on $\mathbb{P}^{1} \backslash\{0,1, \infty\}$. This follows from Proposition 6.15 . Here, $\delta_{u}, \delta_{v}$ are the dihedral symbols corresponding to the chords $\left\{a, a^{\prime}\right\}$, and $\left\{b, b^{\prime}\right\}$. We have

$$
Z^{u, v}=\operatorname{Reg}\left(L_{u, \delta}, v\right)=\operatorname{Reg}(F, 1)=Z^{0,1}\left(\delta_{u}, \delta_{v}\right) .
$$

It follows from the calculations in $\S 5.5$ that the coefficients of $Z^{u, v}$ lie in $\mathcal{Z}$.

As an example, consider the case $\mathfrak{M}_{0,5}$. Then $X_{5, \delta}$ is a pentagon with vertices $v_{1}, v_{2}, . ., v_{5}$ in order. It follows from (6.35) that $Z^{v_{1} v_{2}} Z^{v_{2} v_{3}} Z^{v_{3} v_{4}} Z^{v_{4} v_{5}} Z^{v_{5} v_{1}}=1$. Applying (6.38), we deduce the pentagonal relation due to Drinfeld [18]:

$$
Z^{0,1}\left(\delta_{25}, \delta_{14}\right) Z^{0,1}\left(\delta_{24}, \delta_{13}\right) Z^{0,1}\left(\delta_{14}, \delta_{35}\right) Z^{0,1}\left(\delta_{13}, \delta_{25}\right) Z^{0,1}\left(\delta_{35}, \delta_{24}\right)=1 \in \widehat{\mathfrak{B}}_{5, \delta}(\mathcal{Z}) .
$$

REMARK 6.21. - One can prove the previous theorem directly using Corollary 6.17. In cubical coordinates, the coefficients of $L_{v, \delta}$ are sums of products of logarithms with the multiple polylogarithms $\operatorname{Li}_{n_{1}, \ldots, n_{r}}\left(\frac{x_{j_{1}} \ldots x_{\ell}}{x_{j_{2}} \ldots x_{\ell}}, \ldots, \frac{x_{j_{r}-1} \ldots x_{\ell}}{x_{j_{r}} \ldots x_{\ell}}, x_{j_{r}} \ldots x_{\ell}\right)$. By taking suitable limits in such coordinate systems, one can deduce that the coefficients of each regularised zeta series are multiple zeta values.

We can compute the monodromy of a solution $L_{v, \delta}(x)$ to (6.22) explicitly in terms of the zeta series defined above. First, let us define

$$
\pi_{1}^{\delta}\left(\mathfrak{M}_{0, S}\right)=\pi_{1}\left(\mathfrak{M}_{0, S}, X_{S, \delta}\right)
$$

to be the fundamental group of $\mathfrak{M}_{0, S}$ relative to the set $X_{S, \delta}$, which can be taken as a base point because it is contractible. For each $\{i, j\} \in \chi_{S, \delta}$, let

$$
\gamma_{i j} \in \pi_{1}^{\delta}\left(\mathfrak{M}_{0, S}\right)
$$


denote a small path which winds once around the face $D_{i j}=\left\{u_{i j}=0\right\}$ in the positive direction, i.e., such that

$$
\int_{\gamma_{i j}} \frac{d u_{i j}}{u_{i j}}=+2 \pi i
$$

For each $\{i, j\} \in \chi_{S, \delta}$, let $\mathcal{M}_{i j}$ denote the monodromy operator given by analytic continuation of functions along a loop which is homotopy equivalent to $\gamma_{i j}$. The operators $\mathcal{M}_{i j}$ commute with multiplication and differentiation. It follows that the $\mathcal{M}_{i j}$, for $\{i, j\} \in \chi_{S, \delta}$, act on $L_{v, \delta}(x)$ by right multiplication by constant series.

Proposition 6.22. - Let $\{i, j\} \in \chi_{S, \delta}$, and let $v \in V^{\delta}$ denote any vertex of $\bar{X}_{S, \delta}$. Choose any vertex $w \in V^{\delta}$ which lies on the face $D_{i j}$, i.e., $u_{i j}(w)=0$. Then for all $x \in X_{S, \delta}$,

$$
\mathcal{M}_{i j} L_{v, \delta}(x)=L_{v, \delta}(x) Z^{v, w} e^{2 i \pi \delta_{i j}} Z^{w, v} .
$$

Proof. - By Theorem 6.12,

$$
L_{w, \delta}(x)=f_{w}(x) \prod_{\{k, l\} \in F_{w}} \exp \left(\delta_{k l} \log u_{k l}\right),
$$

where $f_{w}(x)$ is holomorphic in a neighbourhood of $w \in \mathfrak{M}_{0, S}^{\delta}(\mathbb{C})$ which contains the interior of the face $D_{i j}$. By analytic continuation along a small loop $\gamma_{i j}$ which is contained in this neighbourhood and winds once around $D_{i j}$, we deduce that

$$
\mathcal{M}_{i j} L_{w, \delta}(x)=L_{w, \delta}(x) \exp \left(2 i \pi \delta_{i j}\right) .
$$

It follows from the definition of the zeta series that $L_{v, \delta}(x)=L_{w, \delta}(x) Z^{w, v}$ for all $x \in X_{S, \delta}$. Since the quotient $\left(L_{v, \delta}(x)\right)^{-1} L_{w, \delta}(x) Z^{w, v}$ is the constant function 1 , which is single-valued, the same equation must also hold for all $x$ in the universal covering space of $\mathfrak{M}_{0, S}(\mathbb{C})$. Therefore,

$$
\mathcal{M}_{i j} L_{v, \delta}(x)=\mathcal{M}_{i j} L_{w, \delta}(x) Z^{w, v}=L_{w, \delta}(x) e^{2 i \pi \delta_{i j}} Z^{w, v}=L_{v, \delta}(x) Z^{v, w} e^{2 i \pi \delta_{i j}} Z^{w, v} .
$$

The previous lemma holds for any pair of vertices $w, w^{\prime} \in V^{\delta}$ which meets $D_{i j}$. We immediately deduce that the following identity holds in $\widehat{\mathfrak{B}}_{S, \delta}(\mathbb{C})$ :

$$
Z^{v, w} e^{2 i \pi \delta_{i j}} Z^{w, v}=Z^{v, w^{\prime}} e^{2 i \pi \delta_{i j}} Z^{w^{\prime}, v} .
$$

This identity in fact follows from the commutation relation (6.18). It follows from the previous theorem that the monodromy of $\mathfrak{M}_{0, S}$ can be completely expressed in terms of multiple zeta values, and the constant $2 \pi i$.

COROLlary 6.23. - The monodromy ring of $\mathfrak{M}_{0, S}$ is $\mathcal{Z}[2 \pi i]$. 


\subsection{Regularisation of polylogarithms on $\mathfrak{M}_{0, S}$}

Definition 6.24. - For any $v \in V^{\delta}$, let $L^{v, \delta}\left(\mathfrak{M}_{0, S}\right)$ denote the $\mathcal{O}\left(\mathfrak{M}_{0, S}\right)$-module generated by the coefficients of the solution $L_{v, \delta}$ to (6.22) given by Theorem 6.12. Now let us define

$$
L_{\mathcal{Z}}^{\delta}\left(\mathfrak{M}_{0, S}\right)=L^{v, \delta}\left(\mathfrak{M}_{0, S}\right) \otimes_{\mathbb{Q}} \mathcal{Z} .
$$

It does not depend, up to isomorphism, on the choice of the vertex $v$ by Lemma 6.19 and Theorem 6.20. We write $\Omega^{k} L^{v, \delta}\left(\mathfrak{M}_{0, S}\right)=L^{v, \delta}\left(\mathfrak{M}_{0, S}\right) \otimes_{\mathcal{O}\left(\mathfrak{M}_{0, S}\right)} \Omega^{k}\left(\mathfrak{M}_{0, S}\right)$ and $\Omega^{k} L_{\mathcal{Z}}^{\delta}\left(\mathfrak{M}_{0, S}\right)=\Omega^{k} L^{v, \delta}\left(\mathfrak{M}_{0, S}\right) \otimes_{\mathbb{Q}} \mathcal{Z}$, for $k \geq 0$.

Since $\mathcal{Z}$ is filtered by the weight, we deduce a natural weight filtration on $L_{\mathcal{Z}}^{\delta}\left(\mathfrak{M}_{0, S}\right)$ which we denote by $W \cdot$. It follows immediately that any dihedral symmetry $\sigma \in D_{2 n}$ of the $n$-gon $(S, \delta)$ induces an isomorphism of filtered algebras

$$
\sigma_{*}: L_{\mathcal{Z}}^{\delta}\left(\mathfrak{M}_{0, S}\right) \stackrel{\sim}{\rightarrow} L_{\mathcal{Z}}^{\delta}\left(\mathfrak{M}_{0, S}\right) .
$$

Each algebra $L^{v, \delta}\left(\mathfrak{M}_{0, S}\right)$ is in fact a graded Hopf algebra $(\S 6.7)$, although we lose the grading when we pass to $L_{\mathcal{Z}}^{\delta}\left(\mathfrak{M}_{0, S}\right)$, because it is not yet known whether $\mathcal{Z}$ is graded by the weight. The following theorem shows that the function theory of multiple polylogarithms is dictated by the geometry of the Stasheff polytopes $\bar{X}_{S, \delta}$.

Theorem 6.25. - Let $\{i, j\} \in \chi_{S, \delta}$. For any function $f \in L_{\mathcal{Z}}^{\delta}\left(\mathfrak{M}_{0, S}\right)$, let $\operatorname{Reg}\left(f, D_{i j}\right)$ denote the regularised restriction of $f$ to the divisor $D_{i j}$, which maps not only logarithmic, but also polar singularities to zero. As in Lemma 2.6, let $T_{1} \cup T_{2}=S$ denote the partition corresponding to the chord $e=\{i, j\}$, such that

$$
D_{i j} \cong \overline{\mathfrak{M}}_{0, T_{1} \cup e}^{\delta_{1}} \times \overline{\mathfrak{M}}_{0, T_{2} \cup e}^{\delta_{2}} .
$$

Then there is an isomorphism of filtered algebras:

$$
\operatorname{Reg}\left(L_{\mathcal{Z}}^{\delta}\left(\mathfrak{M}_{0, S}\right), D_{i j}\right) \cong L_{\mathcal{Z}}^{\delta_{1}}\left(\mathfrak{M}_{0, T_{1}}\right) \otimes_{\mathcal{Z}} L_{\mathcal{Z}}^{\delta_{2}}\left(\mathfrak{M}_{0, T_{2}}\right)
$$

Proof. - Let us choose any vertex $v \in V^{\delta}$ such that $u_{i j}(v)=0$. The algebra $L^{v, \delta}\left(\mathfrak{M}_{0, S}\right) \otimes_{\mathbb{Q}} \mathcal{Z}$ is generated by the coefficients of the generating series $L_{v, \delta}(x)$ over $\mathcal{Z}$. By Proposition 6.15 , there is a decomposition

$$
L_{v, \delta}=h L_{1} L_{2}
$$

where $L_{1}, L_{2}$ can be viewed as solutions of (6.22) on $\mathfrak{M}_{0, T_{1}}$ and $\mathfrak{M}_{0, T_{2}}$ respectively, and do not depend on $u_{i j}$. Since the series $h \exp \left(-\delta_{i j} \log u_{i j}\right)$ is holomorphic in $u_{i j}$ and is the constant function 1 along $D_{i j}=\left\{u_{i j}=0\right\}$, we have $\operatorname{Reg}\left(h, D_{i j}\right)=1$. Therefore

$$
\operatorname{Reg}\left(L_{v, \delta}, D_{i j}\right)=L_{1} L_{2} .
$$

Likewise, for any coefficient $f$ of $L_{v, \delta}$, and any $k \in \mathbb{Z}$,

$$
\operatorname{Reg}\left(\frac{f}{u_{i j}^{k}}, D_{i j}\right) \in L^{v_{1}, \delta_{1}}\left(\mathfrak{M}_{0, T_{1}}\right) \otimes_{\mathbb{Q}} L^{v_{2}, \delta_{2}}\left(\mathfrak{M}_{0, T_{2}}\right)
$$

where $v_{1}, v_{2}$ are the images of $v$ defined in $\S 6.5$, and $\delta_{1}, \delta_{2}$ are the induced dihedral structures on $T_{1}, T_{2}$. This proves that there is an isomorphism of filtered algebras $\operatorname{Reg}\left(L^{v, \delta}\left(\mathfrak{M}_{0, S}\right), D_{i j}\right) \cong L^{v_{1}, \delta_{1}}\left(\mathfrak{M}_{0, T_{1}}\right) \otimes_{\mathbb{Q}} L^{v_{2}, \delta_{2}}\left(\mathfrak{M}_{0, T_{2}}\right)$. On taking the tensor product with $\mathcal{Z}$, we obtain the statement of the theorem. 
The theorem states that if we restrict a multiple polylogarithm of weight $m$ to the divisor $u_{i j}=0$, then we obtain a linear combination of products of multiple zeta values and multiple polylogarithms such that the total weight is at most $m$.

\subsection{The regularised realisation of polylogarithms}

Let $S=\left\{s_{1}, \ldots, s_{n}\right\}$ with the obvious dihedral structure $\delta$, and let $v \in V^{\delta}$. We can now define a realisation of $B\left(\mathfrak{M}_{0, S}\right)$ which is regularised at $v$. Let us first suppose that $v$ corresponds to the vertex $x_{1}=x_{2}=\cdots=x_{\ell}=0$ in cubical coordinates, in order to exploit the decomposition of $B\left(\mathfrak{M}_{0, S}\right)$ as a product of shuffle algebras. The corresponding triangulation of the $n$-gon $(S, \delta)$ consists of all chords $\{\{2,4\}, \ldots,\{2, n\}\}$. The projection map onto $x_{\ell}=0$ gives a fibration

$$
\mathfrak{M}_{0,\left\{s_{1}, \ldots, s_{n}\right\}} \longrightarrow \mathfrak{M}_{0,\left\{s_{2}, \ldots, s_{n}\right\}} .
$$

Correspondingly, we proved that there is a decomposition

$$
B\left(\mathfrak{M}_{0, S}\right)=B\left(\mathfrak{M}_{0, S^{\prime}}\right) \otimes_{\mathcal{O}\left(\mathfrak{M}_{0, S^{\prime}}\right)} B_{\mathfrak{M}_{0, S^{\prime}}}\left(\mathbb{P}^{1} \backslash \Sigma\right),
$$

where $S^{\prime}=\left\{s_{2}, \ldots, s_{n}\right\}$, and $\Sigma$ is given in $\S 5.4$. Now let $v^{\prime}$ denote the vertex corresponding to the restricted triangulation $\alpha^{\prime}$ of $S^{\prime}$ with induced dihedral structure $\delta^{\prime}$. By Proposition 6.15, there is a decomposition $L_{v, \delta}=h L_{v^{\prime}, \delta^{\prime}}$, where $h$ is a hyperlogarithm equation on $\mathbb{P}^{1} \backslash \Sigma$ in the variable $x_{\ell}$. We deduce that

$$
L^{v, \delta}\left(\mathfrak{M}_{0, S}\right)=L^{v^{\prime}, \delta^{\prime}}\left(\mathfrak{M}_{0, S^{\prime}}\right) \otimes_{\mathcal{O}\left(\mathfrak{M}_{0, S^{\prime}}\right)} L_{\mathfrak{M}_{0, S^{\prime}}}\left(\mathbb{P}^{1} \backslash \Sigma\right),
$$

where $L_{\mathfrak{M}_{0, S^{\prime}}}\left(\mathbb{P}^{1} \backslash \Sigma\right)$ denotes the $\mathcal{O}\left(\mathfrak{M}_{0, S^{\prime}}\right)$-algebra generated by the coefficients of $h$. From the realisation $(5.8)$ we obtain a realisation:

$$
\rho_{S^{\prime}}: B_{\mathfrak{M}_{0, S^{\prime}}}\left(\mathbb{P}^{1} \backslash \Sigma\right) \stackrel{\sim}{\longrightarrow} L_{\mathfrak{M}_{0, S^{\prime}}}\left(\mathbb{P}^{1} \backslash \Sigma\right),
$$

which is regularised at $x_{\ell}=0$. It is an isomorphism of graded $\mathcal{O}\left(\mathfrak{M}_{0, S^{\prime}}\right)\left[\partial / \partial x_{\ell}\right]$-algebras. If we iterate this argument, we obtain two analogous decompositions

$$
\begin{aligned}
B\left(\mathfrak{M}_{0, S}\right) & =\bigotimes_{1 \leq i \leq \ell} B_{\mathfrak{M}_{0, S_{i}}}\left(\mathbb{P}^{1} \backslash \Sigma_{i}\right) \\
L^{v, \delta}\left(\mathfrak{M}_{0, S}\right) & =\bigotimes_{1 \leq i \leq \ell} L_{\mathfrak{M}_{0, S^{\prime}}}\left(\mathbb{P}^{1} \backslash \Sigma_{i}\right),
\end{aligned}
$$

for some subsets $S_{1} \subsetneq S_{2} \subsetneq \cdots \subsetneq S_{\ell} \subsetneq S$ where $\left|S_{1}\right|=3$, and $\Sigma_{i} \cong S_{i}$. Taking the tensor product of the fibre-wise isomorphisms (6.43), we obtain a map

$$
\rho_{v, \delta}: B\left(\mathfrak{M}_{0, S}\right) \longrightarrow L^{v, \delta}\left(\mathfrak{M}_{0, S}\right) .
$$

THEOREM 6.26. - The map $\rho_{v, \delta}$ is an isomorphism of differential graded algebras. It follows that every $\mathcal{O}\left(\mathfrak{M}_{0, S}\right)$-differential subalgebra of $L^{v, \delta}\left(\mathfrak{M}_{0, S}\right)$ is differentially simple, and that $L^{v, \delta}\left(\mathfrak{M}_{0, S}\right)$ is a polynomial algebra. Furthermore,

$$
H^{0}\left(L^{v, \delta}\left(\mathfrak{M}_{0, S}\right)\right)=\mathbb{Q} \quad \text { and } \quad H^{i}\left(L^{v, \delta}\left(\mathfrak{M}_{0, S}\right)\right)=0 \quad \text { for all } i \geq 1 .
$$

The primitive of a closed form $f \in W^{b} \Omega^{k} L^{v, \delta}\left(\mathfrak{M}_{0, S}\right)$ is of weight at most $b+1$. 
Proof. - The proof of Theorem 3.38 implies that the differential structure of the algebras $B\left(\mathfrak{M}_{0, S}\right)$ and $L^{v, \delta}\left(\mathfrak{M}_{0, S}\right)$ are uniquely determined from the tensor decompositions (6.44), since we have a fixed base point at infinity corresponding to $v$. It follows that $\rho_{v, \delta}$ is a map of differential graded algebras. The fact that it is an isomorphism then follows immediately from Corollary 3.13. The rest of the theorem is a consequence of Theorem 3.26 and Corollary 3.40 .

We obtain a similar decomposition for every vertex $v \in V^{\delta}$.

COROLlaRY 6.27. - For each $v \in V^{\delta}$, there is a canonical realisation

$$
\rho_{v, \delta}: B\left(\mathfrak{M}_{0, S}\right) \longrightarrow L^{v, \delta}\left(\mathfrak{M}_{0, S}\right)
$$

which is regularised at the vertex $v$.

The map $\rho_{v, \delta}$ can be defined directly as follows. Recall from $\S 6.2$ that there is a decomposition $B\left(\mathfrak{M}_{0, S}\right) \cong B_{v, \delta}\left(\mathfrak{M}_{0, S}\right) \otimes \mathbb{Q}\left[\left[\omega_{i_{1} j_{1}}\right], \ldots,\left[\omega_{i_{\ell} j_{\ell}}\right]\right]$ into convergent and non-convergent words, where $\left\{i_{1}, j_{1}\right\}, \ldots,\left\{i_{\ell}, j_{\ell}\right\}$ are the set of chords in the triangulation of the $n$-gon corresponding to $v$. Then $\rho_{v, \delta}$ is the unique homomorphism such that

$$
\begin{aligned}
\rho_{v, \delta}\left(\left[\omega_{i_{k} j_{k}}\right]\right) & =\log u_{i_{k} j_{k}} \quad \text { for all } 1 \leq k \leq \ell, \\
\rho_{v, \delta}\left(\sum_{I} c_{I}\left[\omega_{i_{1}}|\ldots| \omega_{i_{n}}\right]\right) & =\sum_{I} c_{I} \int_{\gamma} \omega_{i_{n}} \ldots \omega_{i_{1}}
\end{aligned}
$$

for all $\sum_{I} c_{I}\left[\omega_{i_{1}}|\ldots| \omega_{i_{n}}\right] \in B_{v, \delta}\left(\mathfrak{M}_{0, S}\right)$, where $\gamma$ is a smooth path such that $\gamma(0)=v$ and $\gamma(1)=z \in \mathfrak{M}_{0, S}(\mathbb{C})$. Such an iterated integral converges, since $B_{v, \delta}\left(\mathfrak{M}_{0, S}\right)$ is spanned by the set of integrable words no element of which ever ends in a symbol $\omega_{i_{k} j_{k}}$ for $1 \leq k \leq \ell$. The integrability condition (3.8) ensures that it only depends on the homotopy class of $\gamma$ and therefore defines a multi-valued function on $\mathfrak{M}_{0, S}(\mathbb{C})$. Correspondingly, there is a decomposition

$$
L^{v, \delta}\left(\mathfrak{M}_{0, S}\right)=L_{c}^{v, \delta}\left(\mathfrak{M}_{0, S}\right) \otimes_{\mathbb{Q}} \mathbb{Q}\left[\log u_{i_{k} j_{k}}: 1 \leq k \leq \ell\right],
$$

where $L_{c}^{v, \delta}\left(\mathfrak{M}_{0, S}\right)=\rho_{v, \delta}\left(B_{v, \delta}\left(\mathfrak{M}_{0, S}\right)\right)$ is the algebra generated by the coefficients of $f_{v, \delta}$ (defined in Theorem 6.12). They are holomorphic in a neighbourhood of $v$.

\section{Period integrals on $\overline{\mathfrak{M}}_{0, n}(\mathbb{R})$ and generalised shuffle products}

Given a regular algebraic $n-3$-form on $\mathfrak{M}_{0, S}$, we give necessary and sufficient conditions for its integral over a fundamental cell $X_{S, \delta}$ to converge. We obtain a formula for the order of vanishing of any such form along any given divisor on $\overline{\mathfrak{M}}_{0, S} \backslash \mathfrak{M}_{0, S}$. Finally, we show how the double shuffle relations for multiple zeta values are a special case of generalised multiplicative structures on the set of all period integrals. 
7.1. - The set of all regular algebraic $\ell$-forms on $\mathfrak{M}_{0, S}$ can be written in terms of a canonical dihedrally-invariant form which we construct as follows. Let $\delta$ be a fixed dihedral structure on $S$, and correspondingly, write $S=\left\{s_{1}, \ldots, s_{n}\right\}$. First we define the following form on $\left(\mathbb{P}^{1}\right)_{*}^{n}$, where the indices are taken modulo $n$ :

$$
\widetilde{\omega}_{S, \delta}=\bigwedge_{j=1}^{n} \frac{d z_{j}}{z_{j}-z_{j+2}}
$$

The forms $\widetilde{\omega}_{S, \delta}$ are $\mathrm{PSL}_{2}(\mathbb{C})$-invariant, since if we set

$$
z_{i}^{\prime}=\frac{\alpha z_{i}+\beta}{\gamma z_{i}+\delta}, \quad \text { for } 1 \leq i \leq n, \quad \text { where } \quad\left(\begin{array}{l}
\alpha \beta \\
\gamma \delta
\end{array}\right) \in \mathrm{PSL}_{2}(\mathbb{C})
$$

then $d z_{i}^{\prime}=\left(\gamma z_{i}+\delta\right)^{-2} d z_{i}$ and $z_{i}^{\prime}-z_{j}^{\prime}=\left(\gamma z_{i}+\delta\right)^{-1}\left(\gamma z_{j}+\delta\right)^{-1}\left(z_{i}-z_{j}\right)$, and therefore $\widetilde{\omega}_{S, \delta}$ is unchanged on replacing each $z_{i}$ by $z_{i}^{\prime}$. In order to define a form on $\mathfrak{M}_{0, S}$, consider the quotient map $p:\left(\mathbb{P}_{*}^{1}\right)^{n} \rightarrow \mathfrak{M}_{0, S}$, which has fibres $\mathrm{PSL}_{2}$. Let $v$ denote a fixed non-zero algebraic invariant 3 -form on $\mathrm{PSL}_{2}(\mathbb{C})$ which is defined over $\mathbb{Q}$. This is uniquely determined up to a non-zero rational multiple. Then there exists a unique form $\omega_{S, \delta}$ on $\mathfrak{M}_{0, S}$ such that

$$
p^{*}\left(\omega_{S, \delta}\right) \wedge v=\widetilde{\omega}_{S, \delta}
$$

The form $\omega_{S, \delta}$ is defined over $\mathbb{Q}$, and is $D_{2 n}$-invariant by construction. In simplicial coordinates (2.3), and using the $\mathrm{PSL}_{2}(\mathbb{C})$-invariance of $(7.1)$, we can normalise the rational coefficient of $\omega_{S, \delta}$ such that:

$$
\omega_{S, \delta}=\frac{d t_{1} \wedge \cdots \wedge d t_{\ell}}{t_{2}\left(t_{3}-t_{1}\right)\left(t_{4}-t_{2}\right) \cdots\left(t_{\ell}-t_{\ell-2}\right)\left(1-t_{\ell-1}\right)},
$$

if $\ell \geq 2$, and $\omega_{S, \delta}=d t_{1}$ if $\ell=1$. In dihedral coordinates, one has $\omega_{S, \delta}=d u_{24}$ if $\ell=1$, and if $\ell \geq 2$, one can write (7.2) using (2.9) as follows:

$$
\omega_{S, \delta}=\frac{d u_{24} \wedge d u_{25} \wedge \cdots \wedge d u_{2 n-1} \wedge d u_{2 n}}{\left(1-u_{24} u_{25}\right)\left(1-u_{25} u_{26}\right) \ldots\left(1-u_{2 n-1} u_{2 n}\right)} .
$$

The latter representation is not unique because of the various relations between the functions $u_{i j}$ and their differentials. The form $\omega_{S, \delta}$ clearly defines a meromorphic form on the compactification $\overline{\mathfrak{M}}_{0, S}$. For any boundary divisor $D \subset \overline{\mathfrak{M}}_{0, S} \backslash \mathfrak{M}_{0, S}$ we denote by $\operatorname{ord}_{D} \omega_{S, \delta}$ the order of vanishing of $\omega_{S, \delta}$ along $D$.

Lemma 7.1. - The form $\omega_{S, \delta}$ has neither zeros nor poles on $\mathfrak{M}_{0, S}^{\delta} \backslash \mathfrak{M}_{0, S}$.

Proof. - In cubical coordinates, $\omega_{S, \delta}$ has the representation:

$$
\omega_{S, \delta}=\frac{d x_{1} \wedge \cdots \wedge d x_{\ell}}{\left(1-x_{1} x_{2}\right) \ldots\left(1-x_{\ell-1} x_{\ell}\right)} .
$$

It is clear that $\omega_{S, \delta}$ is not identically zero nor infinite along the divisors $x_{i}=0$, for $1 \leq i \leq \ell$. In other words, the order of vanishing of $\omega_{S, \delta}$ is zero along the divisor $u_{2 i}=0$ for each $4 \leq i \leq n$. But since $\omega_{S, \delta}$ is $D_{2 n}$-invariant, it follows that the order of vanishing of $\omega_{S, \delta}$ is zero along all divisors at finite distance $u_{i j}=0$, where $\{i, j\} \in \chi_{S, \delta}$. 
In other words, given any fixed dihedral structure $\delta$ on $S$, we can define $\omega_{S, \delta}$ to be the unique (up to multiplication by $\mathbb{Q}^{\times}$) non-zero volume form on $\mathfrak{M}_{0, S}(\mathbb{R})$ which has no zeros or poles at finite distance. Equivalently, it has no zeros or poles on the boundary of the closed Stasheff polytope $\bar{X}_{S, \delta}$.

It follows from the fact that $\mathcal{O}\left(\mathfrak{M}_{0, S}\right)=\mathbb{Q}\left[u_{i j}^{ \pm 1}:\{i, j\} \in \chi_{S, \delta}\right]$, that every algebraic volume form on $\mathfrak{M}_{0, S}^{\delta}(\mathbb{R})$ can be written as a linear combination of forms

$$
\prod_{\{i, j\} \in \chi_{S, \delta}} u_{i j}^{\alpha_{i j}} \omega_{S, \delta}, \quad \text { where } \alpha_{i j} \in \mathbb{Z} \quad \text { for each }\{i, j\} \in \chi_{S, \delta}
$$

Now suppose that we are given a collection of coefficients $\alpha=\left(\alpha_{i j}\right)_{\{i, j\} \in \chi_{S, \delta}}$ which are all non-negative. We define the following family of period integrals:

$$
I_{S, \delta}\left(\alpha_{i j}\right)=\int_{\bar{X}_{S, \delta}} \prod_{\{i, j\} \in \chi_{S, \delta}} u_{i j}^{\alpha_{i j}} \omega_{S, \delta} .
$$

The integral is finite because each function $u_{i j}$ is continuous and bounded on the compact set $\bar{X}_{S, \delta}$. Since $\omega_{S, \delta}$ is positive on $\bar{X}_{S, \delta}$ and invariant under the action of $D_{2 n}$, it follows that $I_{S, \delta}\left(\alpha_{i j}\right)$ is also positive, and we have a dihedral transformation formula:

$$
I_{S, \delta}\left(\alpha_{i j}\right)=I_{S, \delta}\left(\alpha_{\sigma(i) \sigma(j)}\right) \quad \text { for all } \sigma \in D_{2 n} .
$$

These integrals can be written explicitly in simplicial and cubical coordinates.

Lemma 7.2. - In cubical coordinates, we have the following formula:

$$
I_{S, \delta}\left(\alpha_{i j}\right)=\int_{[0,1]^{\ell}} \prod_{i=1}^{\ell} x_{i}^{a_{i}}\left(1-x_{i}\right)^{b_{i}} \prod_{1 \leq i<j \leq \ell}\left(1-x_{i} x_{i+1} \ldots x_{j}\right)^{c_{i j}} d x_{1} \ldots d x_{\ell},
$$

where the indices $a_{i}, b_{i}, c_{i j} \in \mathbb{Z}$ are given by:

$$
\begin{aligned}
a_{i} & =\alpha_{2 i+3}, \\
b_{i} & =\alpha_{i+2 i+4}, \\
c_{i i+1} & =\alpha_{i+2 i+5}-\alpha_{i+2 i+4}-\alpha_{i+3 i+5}-1, \\
c_{i j} & =\alpha_{i+3 j+3}+\alpha_{i+2 j+4}-\alpha_{i+2 j+3}-\alpha_{i+3 j+4}, \quad \text { if } j \geq i+2 .
\end{aligned}
$$

Proof. - In cubical coordinates, the domain of integration is $\bar{X}_{S, \delta} \cong[0,1]^{\ell}$, and the only factors that occur in the denominator of $\omega_{S, \delta}$ are $\left(1-x_{i} x_{i+1}\right)$ by (7.4). Using the definition of the cross-ratios $u_{i j}$, we can rewrite the function

$$
f=\prod_{\{i, j\} \in \chi_{S, \delta}} u_{i j}^{\alpha_{i j}}= \pm \prod_{1 \leq p<q \leq n}\left(z_{p}-z_{q}\right)^{s_{p q}}
$$

where the indices $s_{p q}$ are given by $s_{p q}=\alpha_{p-1 q}+\alpha_{p q-1}-\alpha_{p-1 q-1}-\alpha_{p q}$, and where we set $\alpha_{i i+1}=\alpha_{i i}=0$. In cubical coordinates, we have $z_{1}=1, z_{2}=\infty, z_{3}=0$, and $z_{i+3}=x_{i} \ldots x_{\ell}$, for $1 \leq i \leq \ell$. If we put the various elements together, we obtain the formulae for $b_{i}$ and $c_{i j}$ given above. The formulae for $a_{i}$ are easily deduced using the fact that $x_{1}=u_{24}, \ldots, x_{\ell}=u_{2 n}$. 
Special sub-families of these integrals were considered in [55], [21], [54], where it was also conjectured that they are expressible in terms of multiple zeta values. It is easy to verify that the change of variables matrix given by (7.9) is invertible over $\mathbb{Z}$.

Similarly, in simplicial coordinates one can verify that

$$
I_{S, \delta}\left(\alpha_{i j}\right)=\int_{\Delta} \prod_{i=1}^{\ell} t_{i}^{a_{i}^{\prime}}\left(1-t_{i}\right)^{b_{i}^{\prime}} \prod_{1 \leq i<j \leq \ell}\left(t_{j}-t_{i}\right)^{c_{i j}^{\prime}} \frac{d t_{1} \ldots d t_{\ell}}{t_{2}\left(t_{3}-t_{1}\right) \ldots\left(t_{\ell}-t_{\ell-2}\right)\left(1-t_{\ell-1}\right)},
$$

where $\Delta=\left\{0<t_{1}<\cdots<t_{\ell}<1\right\}$ denotes the unit simplex, and where

$$
\begin{aligned}
a_{i}^{\prime} & =\alpha_{3 i+2}+\alpha_{2 i+3}-\alpha_{3 i+3}-\alpha_{2 i+2}, & & 1 \leq i \leq \ell \\
b_{i}^{\prime} & =\alpha_{n i+3}+\alpha_{1 i+2}-\alpha_{n i+2}-\alpha_{1 i+3}, & & 1 \leq i \leq \ell \\
c_{i j}^{\prime} & =\alpha_{i+2 j+3}+\alpha_{i+3 j+2}-\alpha_{i+3 j+3}-\alpha_{i+2 j+2}, & & 1 \leq i<j \leq \ell
\end{aligned}
$$

where we set $\alpha_{i i+1}=\alpha_{i i}=0$ as above. Once again, it is not difficult to verify that the corresponding change of variables matrix is invertible over $\mathbb{Z}$ (this is implied by Equation (6.17)).

\subsection{Relative periods and mixed Hodge structures}

Let $n=|S|=\ell+3$, and let $A, B$ denote two sets of divisors at infinity on $\overline{\mathfrak{M}}_{0, S} \backslash \mathfrak{M}_{0, S}$, where we assume that $A \cap B$ is of codimension at least 2, i.e., $A$ and $B$ have no shared irreducible components. Consider the relative cohomology group

$$
H^{\ell}\left(\overline{\mathfrak{M}}_{0, S} \backslash A, B \backslash B \cap A\right),
$$

which has a canonical mixed Hodge structure [12]. Since the divisor $A \cup B$ is globally normal crossing, this can be computed using the techniques of [29], [50], and it is easily verified that it is of Tate type. Goncharov and Manin construct an object in the abelian category of mixed Tate motives $\mathrm{MT}(\mathbb{Q})$ over $\mathbb{Q}[14]$, whose Hodge realisation is the mixed Hodge structure (7.10). They then show that this motive is unramified over $\mathbb{Z}$. We shall write the corresponding motive and mixed Hodge structure with the same symbol, because the Hodge realisation functor is fully faithful over $\mathbb{Q}$ ([14], proposition 2.9). Suppose that we are given a relative homology cycle

$$
\left[\Delta_{B}\right] \in H_{\ell}\left(\overline{\mathfrak{M}}_{0, S}, B\right)
$$

We can assume that this class is represented by a smooth compact real submanifold with corners $\Delta_{B}$ whose codimension- $k$ boundary is contained in the $k$-stratum of $B$. More precisely, if $B$ consists of irreducible components $B_{i}$, for $1 \leq i \leq N$, then

$$
\partial^{k} \Delta_{B}=\Delta_{B} \cap \bigcap_{i_{1}, \ldots, i_{k}} B_{i_{1}} \cap \cdots \cap B_{i_{k}}
$$

which may be empty. Suppose that we are given an algebraic $\ell$-form $\Omega_{A}$ on $\mathfrak{M}_{0, S}$ which is defined over $\mathbb{Q}$ and whose singularities are contained in $A$. Then the relative period integral of $\Omega_{A}$ along $\Delta_{B}$ is defined to be

$$
\int_{\Delta_{B}} \Omega_{A} \in \mathbb{C}
$$


By a version of Cauchy's theorem, this integral is invariant under continuous deformations of $\Delta_{B}$ relative to $B$. We can thus assume that $\Delta_{B}$ is disjoint from $A$, and therefore the integral is bounded, since $\Omega_{A}$ is continuous on $\Delta_{B}$, which is compact. Note that the integral depends on the relative cohomology classes of $\Omega_{A}$ in $H^{\ell}\left(\overline{\mathfrak{M}}_{0, S} \backslash A, B \backslash B \cap A\right)$, and $\Delta_{B}$ in $H_{\ell}\left(\overline{\mathfrak{M}}_{0, S} \backslash A, B \backslash B \cap A\right)$.

Lemma 7.3. - $\operatorname{gr}_{0}^{W} H_{\ell}\left(\overline{\mathfrak{M}}_{0, S}, B\right)$ is spanned by the homology classes of a number of cells $X_{S, \delta}$, where $\delta$ is in a certain set of dihedral structures which depends upon $B$.

Proof. - The relative cohomology group $H_{\ell}\left(\overline{\mathfrak{M}}_{0, S}, B\right)$ can be computed using the spectral sequence of the complex

$$
\overline{\mathfrak{M}}_{0, S} \leftarrow \bigsqcup_{i} B_{i} \leftleftarrows \bigsqcup_{i, j} B_{i, j} \leftleftarrows \cdots \leftleftarrows \bigsqcup_{|I|=\ell} B_{I},
$$

where $B$ is the union of a set of divisors $B_{i}$, and $B_{I}=\bigcap_{i \in I} B_{i}$. The spectral sequence degenerates on the $E^{2}$ level and it follows that

$$
\operatorname{gr}_{0}^{W} H_{\ell}\left(\overline{\mathfrak{M}}_{0, S}, B\right) \cong \operatorname{ker}\left(\bigoplus_{|I|=\ell} H_{0}\left(B_{I}\right) \longrightarrow \bigoplus_{|J|=\ell-1} H_{0}\left(B_{J}\right)\right)
$$

In the simplicial complex defined by $B$, this is just $\operatorname{ker}\left(\mathbb{C}^{p} \rightarrow \mathbb{C}^{e}\right)$ where $p$ is the number of points, i.e., $\ell$-fold intersections of divisors, and $e$ is the number of edges, i.e., $\ell-1$-fold intersections. This also computes the number of independent cells in $\overline{\mathfrak{M}}_{0, S}(\mathbb{R})$ bounded by $B \cap \overline{\mathfrak{M}}_{0, S}(\mathbb{R})$. Since $\mathfrak{M}_{0, S}(\mathbb{R})$ is tesselated by the cells $X_{S, \delta}$ (Lemma 2.26), they must generate $\operatorname{gr}_{0}^{W} H_{\ell}\left(\overline{\mathfrak{M}}_{0, S}, B\right)$.

Lemma 7.4. - Every relative period integral over a union of cells $\bar{X}_{S, \delta_{i}}$ is a $\mathbb{Q}$-linear combination of $I_{S, \delta}\left(\alpha_{i j}\right)$, where the $\alpha_{i j}$ are all non-negative.

Proof. - Fix a dihedral structure $\delta$ on $S$. Any such integral $I$ can be written:

$$
I=\sum_{i=1}^{N} \int_{\bar{X}_{S, \delta_{i}}} \omega=\int_{\bar{X}_{S, \delta}} \sum_{i=1}^{N} \sigma_{i}^{*}(\omega),
$$

where $\omega \in \Omega^{\ell}\left(\mathfrak{M}_{0, S}\right)$ is regular, and where $\sigma_{i}$ is an element of $\mathfrak{S}(S)$ which maps the dihedral structure $\delta_{i}$ onto $\delta$. The right-hand side can be written

$$
I=\int_{\bar{X}_{S, \delta}} f \omega_{S, \delta}
$$

where $f \in \mathbb{Q}\left[u_{i j}, u_{i j}^{-1}\right]$ is a regular function on $\mathfrak{M}_{0, S}$. Note that by Lemma 4.9 this integral converges absolutely if and only if $f \omega_{S, \delta}$ has no poles along $\partial \bar{X}_{S, \delta}$. Since $\partial \bar{X}_{S, \delta}$ is the union of divisors $D_{i j}=\left\{u_{i j}=0\right\}$, and since $\operatorname{ord}_{D_{i j}} f \omega_{S, \delta}=\operatorname{ord}_{D_{i j}} f$ (by Lemma 7.1), this implies that $f \in \mathbb{Q}\left[u_{i j}\right]$. Since $f$ is a polynomial in the $u_{i j}$, it can be written as a linear combination of monomials with positive exponents, or in other words, $I$ is a finite $\mathbb{Q}$-linear combination of integrals $I_{S, \delta}\left(\alpha_{i j}\right)$, with $\alpha_{i j}$ all non-negative. 
In order to rephrase the above in motivic terms, we need to recall the notion of framings from [25, 26], [5]. Let $m \geq 0$ denote an integer. An $m$-framing on a mixed Tate motive (or its Hodge realisation) is given by two morphisms

$$
v: \mathbb{Q}(-m) \rightarrow \operatorname{gr}_{2 m}^{W} M \quad \text { and } \quad f: \mathbb{Q}(0) \rightarrow\left(\operatorname{gr}_{0}^{W} M\right)^{\vee} .
$$

A morphism between two framed mixed Tate motives $(M, v, f)$ and $\left(M^{\prime}, v^{\prime}, f^{\prime}\right)$ is a morphism $M \rightarrow M^{\prime}$ which respects the framings. This generates an equivalence relation, and one can show that the equivalence classes of $m$-framed mixed Tate motives form an abelian group. The framings on the motive (7.10) were defined in [27] as follows. There are isomorphisms

$$
\begin{aligned}
\operatorname{gr}_{0}^{W} H_{\ell}\left(\overline{\mathfrak{M}}_{0, S} \backslash A, B \backslash B \cap A\right) & \cong \operatorname{gr}_{0}^{W} H_{\ell}\left(\overline{\mathfrak{M}}_{0, S}, B\right), \\
\operatorname{gr}_{2 \ell}^{W} H^{\ell}\left(\overline{\mathfrak{M}}_{0, S} \backslash A, B \backslash B \cap A\right) & \cong \operatorname{gr}_{2 \ell}^{W} H^{\ell}\left(\overline{\mathfrak{M}}_{0, S} \backslash A\right) .
\end{aligned}
$$

Therefore, the classes $\left[\Delta_{B}\right] \in \operatorname{gr}_{0}^{W} H_{\ell}\left(\overline{\mathfrak{M}}_{0, S}, B\right)$ and $\left[\Omega_{A}\right] \in \operatorname{gr}_{2 \ell}^{W} H^{\ell}\left(\overline{\mathfrak{M}}_{0, S} \backslash A\right)$ define an $\ell$-framing on (7.10). Note that these framings could be zero.

We introduce a simplified variant of the above motives. Let $\delta$ denote a fixed dihedral structure on $S$ and let $D_{\delta}$ denote the set of divisors at finite distance in $\mathfrak{M}_{0, S}^{\delta}$. These are the affine varieties which bound the fundamental cell $\bar{X}_{S, \delta}$. Let $\omega \in \Omega^{\ell}\left(\mathfrak{M}_{0, S}\right)$ denote an algebraic $\ell$-form with no singularities along $D_{\delta}$, which is defined over $\mathbb{Q}$. Let us define the $\ell$-framed mixed Tate motive:

$$
m_{S, \delta}(\omega)=\left(H^{\ell}\left(\mathfrak{M}_{0, S}^{\delta}, D_{\delta}\right),\left[\bar{X}_{S, \delta}\right],[\omega]\right),
$$

equipped with the framings given by the class of the fundamental cell $\Delta_{B}=\bar{X}_{S, \delta}$, and the class of $\omega$. The framed motives $m_{S, \delta}(\omega)$ are more convenient to work with because the varieties $\mathfrak{M}_{0, S}^{\delta}$ are affine, and we do not need to keep track of the divisor data at infinity. Lemmas 7.3 and 7.4 imply that the framed mixed Tate motive $\left(H^{\ell}\left(\overline{\mathfrak{M}}_{0, S} \backslash A, B \backslash B \cap A\right),\left[\Delta_{B}\right],\left[\Omega_{A}\right]\right)$, is equivalent to a linear combination of motives:

$$
m_{S, \delta}\left(f \omega_{S, \delta}\right), \quad \text { where } f=\prod_{\{i, j\} \in \chi_{S, \delta}} u_{i j}^{\alpha_{i j}} .
$$

The equivalence is given by natural inclusion maps between moduli spaces, the action of the symmetric group, and the additivity of framed objects with respect to their framings.

\subsection{Formulae for the divisor of singularities}

In order to compute the divisor of singularities of an arbitrary form (7.5), it suffices to compute the order of the canonical form $\omega_{S, \delta}$ along each divisor at infinity. This is easily done by exploiting the action of the symmetric group.

Proposition 7.5. - Let $|S|=n=\ell+3$, and let $D$ denote the divisor given by the stable partition $S^{1} \cup S^{2}=S$ (Proposition 2.35). Then

$$
\operatorname{ord} \omega_{S, \delta}=\frac{\ell-1}{2}-\frac{1}{2} \sum_{i \in \mathbb{Z} / n \mathbb{Z}} \mathbb{I}_{D}(i, i+2),
$$

where the notation $\mathbb{I}_{D}$ is defined by Equation (2.45) in $\S 2.8$. 
Proof. - Let $k \geq 2$ denote the number of elements in $S^{1}$. Let $\sigma$ denote a permutation $\sigma \in \mathfrak{S}(n)$ such that $\sigma^{-1}\left(S^{1}\right)=\{1,2, \ldots, k\}$. By (7.1), we have

$$
\sigma^{*}\left(\widetilde{\omega}_{S, \delta}\right)= \pm \prod_{i \in \mathbb{Z} / n \mathbb{Z}}\left(\frac{z_{i}-z_{i+2}}{z_{\sigma(i)}-z_{\sigma(i+2)}}\right) \widetilde{\omega}_{S, \delta}
$$

By passing to the quotient $p:\left(\mathbb{P}^{1}\right)_{*}^{S} \rightarrow \mathfrak{M}_{0, S}$, we have

$$
\operatorname{ord}_{D} \omega_{S, \delta}=\operatorname{ord}_{D} \sigma^{*}\left(\omega_{S, \delta}\right)-\operatorname{ord}_{D} f
$$

where the function

$$
f=\prod_{i \in \mathbb{Z} / n \mathbb{Z}}\left(\frac{z_{i}-z_{i+2}}{z_{\sigma(i)}-z_{\sigma(i+2)}}\right)
$$

is homogeneous and $\mathrm{PSL}_{2}(\mathbb{C})$-invariant by the remarks in $\S 7.1$. It can therefore be written as a product of cross-ratios and is a well-defined function on $\mathfrak{M}_{0, S}$. Now $\operatorname{ord}_{D} \sigma^{*}\left(\omega_{S, \delta}\right)=$ $\operatorname{ord}_{\{1, \ldots, k\}} \omega_{S, \delta}=0$, since the divisor given by the stable partition $\{1, \ldots, k\} \cup\{k+1, \ldots, n\}$ is $D_{k n}=\left\{u_{k n}=0\right\}$, and we know by Lemma 7.1 that $\omega_{S, \delta}$ has no zeros or poles at finite distance. Therefore

$$
\operatorname{ord}_{D} \omega_{S, \delta}=-\operatorname{ord}_{D} f
$$

and it suffices to compute the zeros and poles of $f$. Recall from Corollary 2.36 that

$$
\operatorname{ord}_{D} \frac{\left(z_{i}-z_{k}\right)\left(z_{j}-z_{l}\right)}{\left(z_{i}-z_{l}\right)\left(z_{j}-z_{k}\right)}=\frac{1}{2}\left[\mathbb{I}_{D}(i, k)+\mathbb{I}_{D}(j, l)-\mathbb{I}_{D}(i, l)-\mathbb{I}_{D}(j, k)\right] .
$$

We deduce that

$$
\operatorname{ord}_{D} f=\frac{1}{2} \sum_{i \in \mathbb{Z} / n \mathbb{Z}}\left(\mathbb{I}_{D}(i, i+2)-\mathbb{I}_{D}(\sigma(i), \sigma(i+2))\right) .
$$

But $\{\sigma(i), \sigma(i+2)\} \subset S^{1}$ if and only if $\{i, i+2\} \subset\{1, \ldots, k\}$. The number of such pairs is exactly $k-2$. Likewise, the number of $i$ such that $\{\sigma(i), \sigma(i+2)\} \subset S^{2}$ is $n-k-2$. It follows that the second quantity in the sum directly above is $n-4=\ell-1$. This completes the proof on substituting into (7.14).

We immediately deduce the following formula for the order of vanishing of an arbitrary form along any divisor $D \subset \overline{\mathfrak{M}}_{0, S} \backslash \mathfrak{M}_{0, S}$. Let

$$
f=\prod_{\{i, j\} \in \chi_{S, \delta}} u_{i j}^{\alpha_{i j}}, \quad \alpha_{i j} \in \mathbb{Z}
$$

Corollary 7.6. - Let $D$ and $f$ be as above. Then

$$
\begin{aligned}
2 \operatorname{ord}_{D} f \omega_{S, \delta}= & \sum_{\{i, j\} \in \chi_{S, \delta}} \alpha_{i j}\left[\mathbb{I}_{D}(i, j+1)+\mathbb{I}_{D}(i+1, j)-\mathbb{I}_{D}(i, j)-\mathbb{I}_{D}(i+1, j+1)\right] \\
& +(\ell-1)-\sum_{i \in \mathbb{Z} / n \mathbb{Z}} \mathbb{I}_{D}(i, i+2) .
\end{aligned}
$$

Proof. - This follows immediately from the additivity of $\operatorname{ord}_{D}$ and the fact that $2 \operatorname{ord}_{D} u_{i j}=2 \operatorname{ord}_{D}[i i+1 \mid j+1 j]=\mathbb{I}_{D}(i, j+1)+\mathbb{I}_{D}(i+1, j)-\mathbb{I}_{D}(i, j)-\mathbb{I}_{D}(i+1, j+1)$. 
Note that along each divisor at finite distance $D_{i j}=\left\{u_{i j}=0\right\}$, where $\{i, j\} \in \chi_{S, \delta}$, we clearly have $\operatorname{ord}_{D}(f)=\alpha_{i j}$. In total, there are as many boundary divisors $D \in \overline{\mathfrak{M}}_{0, S}$ as there are partitions of $S$ into two sets, each containing at least two elements. These number $2^{n-1}-n-1$, but there are only $n(n-3) / 2$ parameters $\alpha_{i j}$, which implies that there are many relations between the quantities $\operatorname{ord}_{D} f$, for varying $D$. The following lemma gives an alternative approach for computing the orders of functions along divisors.

Lemma 7.7. - Let $D$ be the divisor of $\overline{\mathfrak{M}}_{0, S} \backslash \mathfrak{M}_{0, S}$ corresponding to a stable partition $S_{1} \cup S_{2}=S$. For each two-element subset $T=\left\{s_{i}, s_{j}\right\} \subset S_{1}$, let $D_{T}$ denote the divisor given by the partition $T$ and its complement $S \backslash T$. Then for any function $f \in \mathbb{Q}\left(\mathfrak{M}_{0, S}\right)$,

$$
\operatorname{ord}_{D} f=\sum_{T \subset S_{1},|T|=2} \operatorname{ord}_{D_{T}} f .
$$

Proof. - It suffices to verify the formula for the function $f=u_{i j}$, where $\{i, j\} \in \chi_{S, \delta}$, since it is compatible with products. By Corollary 2.36,

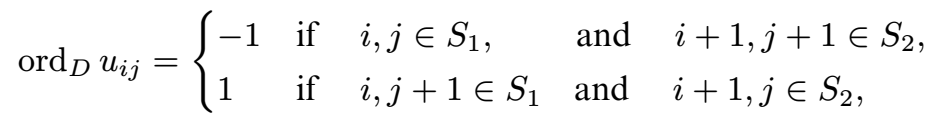

and is 0 otherwise. One can check that the identity holds for two-element subsets of $S_{1} \cap\{i, i+1, j, j+1\}$, from which it follows in general. For example, if $S_{1} \cap\{i, i+1, j, j+1\}=$ $\{i, i+1, j+1\}$, then $\operatorname{ord}_{D} u_{i j}=0$ and this is equal to

$$
\operatorname{ord}_{D_{\{i, i+1\}}} u_{i j}+\operatorname{ord}_{D_{\{i, j+1\}}} u_{i j}+\operatorname{ord}_{D_{\{i+1, j+1\}}} u_{i j}=0+1-1 .
$$

\subsection{Singularities of the Kontsevich multiple zeta value forms}

There is a special set of $\ell$-forms on $\mathfrak{M}_{0, S}$ corresponding to the iterated integral representations of multiple zeta values due to Kontsevich. Let $n \geq 5$. We apply the previous proposition to compute the divisor of singularities of each such form and retrieve one of the results of [27]. Let $\underline{\epsilon}=\left(\epsilon_{1}, \ldots, \epsilon_{\ell}\right)$ where $\epsilon_{1}, \ldots, \epsilon_{\ell} \in\{0,1\}$. We define

$$
\gamma_{i}=3-2 \epsilon_{i} \in\{1,3\}, \quad \text { for } 1 \leq i \leq \ell,
$$

and set

$$
\Omega(\underline{\epsilon})=\left[\begin{array}{llll}
5 & n \mid 3 & 2
\end{array}\right]\left[\begin{array}{lll}
2 & n \mid 1 & 3
\end{array}\right]^{\varepsilon_{\ell}} \prod_{i=1}^{\ell-1}\left[\begin{array}{lll}
i+5 & \gamma_{i} \mid i+3 & 2
\end{array}\right] \omega_{S, \delta} .
$$

The term in the product corresponding to $i=\ell-1$ requires explanation. We define $\left[n+1 \gamma_{\ell-1} \mid n-12\right]=\left[\begin{array}{lll}1 & 3 \mid n-1 & 2\end{array}\right]$ if $\gamma_{\ell-1}=3$, and define it to be 1 if $\gamma_{\ell-1}=1$. We can write this expression in explicit simplicial coordinates (2.3) by setting $z_{1}=1, z_{2}=\infty$ and $z_{3}=0$. If we define $t_{\ell+1}=1$, one can verify using (7.2) that

$$
\Omega(\underline{\epsilon})=\frac{t_{2}}{t_{\ell}}\left(\frac{t_{\ell}}{t_{\ell}-1}\right)^{\varepsilon_{\ell}} \prod_{i=1}^{\ell-1}\left(\frac{t_{i+2}-t_{i}}{\epsilon_{i}-t_{i}}\right) \omega_{S, \delta}=\bigwedge_{i=1}^{\ell} \frac{d t_{i}}{\epsilon_{i}-t_{i}} .
$$


Let $X=\left\{x_{0}, x_{1}\right\}$ be an alphabet with two letters as considered in $\S 5.5$. Assume that $\epsilon_{1}=1$ and $\epsilon_{\ell}=0$, and define a word $w=x_{\epsilon_{\ell}} \ldots x_{\epsilon_{1}} \in x_{0} X^{*} x_{1}$. Let $r_{\epsilon}=\sum_{i=1}^{\ell} \epsilon_{i}$. It follows from (2.38) and a well-known formula for $\zeta(w)$ that

$$
\int_{X_{S, \delta}} \Omega(\underline{\epsilon})=\int_{0<t_{1}<\cdots<t_{\ell}<1} \bigwedge_{i=1}^{\ell} \frac{d t_{i}}{\epsilon_{i}-t_{i}}=(-1)^{\ell-r} \zeta(w)
$$

The integral converges if and only if $\epsilon_{1}=1$ and $\epsilon_{\ell}=0$. It follows that every multiple zeta value of weight $\ell$ occurs as a relative period of $\mathfrak{M}_{0, \ell+3}(\mathbb{R})$ [27].

Lemma 7.8. - Let $\underline{\epsilon}=\left(\epsilon_{1}, \ldots, \epsilon_{\ell}\right)$ with $\epsilon_{i} \in\{0,1\}$ for all $1 \leq i \leq \ell$. Then

$$
2 \operatorname{ord}_{D} \Omega(\underline{\epsilon})=\ell-1+\sum_{i=1}^{\ell}\left[\mathbb{I}_{D}\left(2, \gamma_{i}\right)-\mathbb{I}_{D}\left(i+3, \gamma_{i}\right)\right]-\sum_{k \neq 2} \mathbb{I}_{D}(2, k)-\mathbb{I}_{D}(1,3),
$$

where $\gamma_{i} \in\{1,3\}$ is defined in (7.16).

Proof. - First we assume that $\epsilon_{\ell}=0$, and therefore $\gamma_{\ell}=3$. It follows from (7.17) that

$$
\begin{aligned}
2 \operatorname{ord}_{D} \Omega(\underline{\epsilon})= & \sum_{i=1}^{\ell-1} \mathbb{I}_{D}(i+3, i+5)+\mathbb{I}_{D}\left(2, \gamma_{i}\right)-\mathbb{I}_{D}(2, i+5)-\mathbb{I}_{D}\left(i+3, \gamma_{i}\right) \\
& +\mathbb{I}_{D}(3,5)+\mathbb{I}_{D}(2, n)-\mathbb{I}_{D}(2,5)-\mathbb{I}_{D}(3, n)+\operatorname{ord}_{D} \omega_{S, \delta}
\end{aligned}
$$

and the formula stated above follows on substituting the expression for $\operatorname{ord}_{D} \omega_{S, \delta}$ given in Proposition 7.5. In the case where $\epsilon_{\ell}=1, \gamma_{\ell}=1$, a similar formula for $\Omega(\underline{\epsilon})$ holds except that one must multiply by an extra cross-ratio $[2 n \mid 13]$. This contributes

$$
\mathbb{I}_{D}(2,1)-\mathbb{I}_{D}(n, 1)-\left(\mathbb{I}_{D}(2,3)-\mathbb{I}_{D}(n, 3)\right)
$$

in the expression above, and this is precisely what is required for the formula to hold in this case also.

Let $D \subset \overline{\mathfrak{M}}_{0, S} \backslash \mathfrak{M}_{0, S}$ be the divisor corresponding to a stable partition $S_{1} \cup S_{2}$ of $S$. Then, up to permuting the sets $S_{1}$ and $S_{2}, D$ is one of the following four types, where $A \cup B$ is a partition of $\left\{s_{4}, \ldots, s_{n}\right\}$ :

1. $S_{1}=\left\{s_{1}, s_{2}, s_{3}\right\} \cup A, S_{2}=B$.

2. $S_{1}=\left\{s_{1}, s_{3}\right\} \cup A, \quad S_{2}=\left\{s_{2}\right\} \cup B$.

3. $S_{1}=\left\{s_{1}, s_{2}\right\} \cup A, \quad S_{2}=\left\{s_{3}\right\} \cup B$.

4. $S_{1}=\left\{s_{2}, s_{3}\right\} \cup A, \quad S_{2}=\left\{s_{1}\right\} \cup B$.

Corollary 7.9. - Let $A_{0}=A \cap\left\{s_{i+3}\right.$ for $1 \leq i \leq \ell$ such that $\left.\epsilon_{i}=0\right\}, A_{1}=A \backslash A_{0}$, and define $B_{0}, B_{1}$, similarly. Then, according to each of the cases above,

$$
\operatorname{ord}_{D} \Omega(\underline{\epsilon})= \begin{cases}|B|-2 & \text { if } D \text { is as in case (1), } \\ -1 & \text { if } D \text { is as in case (2), } \\ \left|B_{1}\right|-1 & \text { if } D \text { is as in case (3), } \\ \left|B_{0}\right|-1 & \text { if } D \text { is as in case (4). }\end{cases}
$$


Proof. - In case (1), the formula stated in the previous lemma gives, term by term,

$$
2 \operatorname{ord}_{D}(\Omega(\underline{\epsilon}))=\ell-1+\ell-|A|-(|A|+2)-1,
$$

and the formula follows, since $\ell=|A|+|B|$. In case (2), it gives

$$
2 \operatorname{ord}_{D}(\Omega(\underline{\epsilon}))=\ell-1+0-|A|-|B|-1=-2 .
$$

In case (3), we have $S_{1}=\left\{s_{1}, s_{2}\right\} \cup A_{0} \cup A_{1}$ and $S_{2}=\left\{s_{3}\right\} \cup B_{0} \cup B_{1}$. The formula in the previous lemma gives, term by term:

$$
2 \operatorname{ord}_{D}(\Omega(\underline{\epsilon}))=\ell-1+\left(\left|A_{1}\right|+\left|B_{1}\right|\right)-\left(\left|B_{0}\right|+\left|A_{1}\right|\right)-\left(1+\left|A_{0}\right|+\left|A_{1}\right|\right)-0,
$$

but since $\ell=\left|A_{0}\right|+\left|A_{1}\right|+\left|B_{0}\right|+\left|B_{1}\right|$, this is just $2\left|B_{1}\right|-2$, as required. The formula for case (4) follows by symmetry.

In case (1), we must have $\left|S_{2}\right|=|B| \geq 2$, otherwise the partition $S_{1} \cup S_{2}$ is not stable, so no singularity ever occurs along such a divisor. It follows that the divisors of singularities of $\Omega(\underline{\epsilon})$ are precisely those divisors of type (2), and those of type (3) (resp. (4)) for which $B_{1}$ (resp. $\left.B_{0}\right)$ is empty. Let us set $s_{1}=1, s_{2}=\infty$, and $s_{3}=0$, as usual. Then the divisors of type (2) correspond to the divisors which are called 'type $\infty$ ' in [27]. The divisors of type (3) for which there is a pole are partitions of the form $\{1, \infty\} \cup A$ and $\{0\} \cup B$, where $B=B_{0}$ and hence $B \subset\left\{s_{i+3}: \epsilon_{i}=0\right\}$. These are exactly the divisors of 'type 0' according to [27]. Similarly, our type (4) above corresponds to 'type 1' and the previous result implies Proposition 3.1 of [27]. Note that the above proof only uses the action of the symmetric group and does not use any blow-ups.

\subsection{Generalised products and the double shuffle relations}

In $\S 2.10$ we considered non-degenerate coordinate systems

$$
f=\prod_{i=1}^{k} f_{T_{i}}: \mathfrak{M}_{0, S} \longrightarrow \prod_{i=1}^{k} \mathfrak{M}_{0, T_{i}}
$$

where the sets $T_{i}$ cover $S$ and the dimensions satisfy (2.49). Since $\mathfrak{M}_{0, T_{i}}$ is affine of algebraic dimension $\left|T_{i}\right|-3$, the Künneth formula gives an isomorphism

$$
\bigotimes_{i=1}^{k} H^{\left|T_{i}\right|-3}\left(\mathfrak{M}_{0, T_{i}}\right) \cong H^{|S|-3}\left(\prod_{i=1}^{k} \mathfrak{M}_{0, T_{i}}\right) .
$$

We deduce the existence of a multiplication map for forms:

$$
f^{*}: \bigotimes_{i=1}^{k} H^{\left|T_{i}\right|-3}\left(\mathfrak{M}_{0, T_{i}}\right) \longrightarrow H^{|S|-3}\left(\mathfrak{M}_{0, S}\right)
$$

This in turn gives a product formula for period integrals on the spaces $\mathfrak{M}_{0, S}$. If $S$ has dihedral structure $\delta$, then it induces dihedral structures $\delta_{i}$ on $T_{i}$. Recall that the fundamental domains $\prod_{i=1}^{k} X_{T_{i}, \delta_{i}}$ and $X_{S, \delta}$ are related by the set $G_{f}$ defined in (2.50) via the formula (2.51).

Corollary 7.10. - Let $\omega_{i} \in H^{\left|T_{i}\right|-3}\left(\mathfrak{M}_{0, T_{i}}\right)$, for $1 \leq i \leq k$. Then

$$
\prod_{i=1}^{k} \int_{X_{T_{i}, \delta_{i}}} \omega_{i}=\sum_{\gamma \in G_{f}} \int_{X_{S, \gamma}} f^{*}\left(\omega_{1} \otimes \cdots \otimes \omega_{k}\right) .
$$


It follows that a product of period integrals on real moduli spaces is itself a period of real moduli spaces.

ReMark 7.11. - We know that $f$ extends to give a map $\mathfrak{M}_{0, S}^{\delta} \longrightarrow \prod_{i=1}^{k} \mathfrak{M}_{0, T_{i}}^{\delta_{i}}$. The previous corollary therefore implies the following multiplication formula for the framed mixed Tate motives defined in $\S 7.2$ :

$$
\bigotimes_{i=1}^{k} m_{T_{i}, \delta_{i}}\left(\omega_{i}\right)=\bigoplus_{\gamma \in G_{f}} m_{S, \gamma}\left(f^{*}\left(\omega_{1} \otimes \cdots \otimes \omega_{k}\right)\right) .
$$

We can apply the product formula above to the set of multiple zeta forms $\Omega(\underline{\epsilon})$ defined in $\S 7.4$. As in $\S 5.5$, let $X$ denote an alphabet with two letters $\left\{x_{0}, x_{1}\right\}$. Let $w=x_{\epsilon_{m}} \ldots x_{\epsilon_{1}}$ and $w^{\prime}=x_{\epsilon_{\ell}} \ldots x_{\epsilon_{m+1}}$ denote two words in $x_{0} X^{*} x_{1}$, where $\epsilon_{i} \in\{0,1\}$, such that $\epsilon_{1}=\epsilon_{m+1}=1$ and $\epsilon_{m}=\epsilon_{\ell}=0$. Let us write $\underline{\epsilon}=\left(\epsilon_{1}, \ldots, \epsilon_{m}\right)$ and $\underline{\epsilon}^{\prime}=\left(\epsilon_{m+1}, \ldots, \epsilon_{\ell}\right)$. Recall the simplicial product map defined in $\S 2.10$ :

$$
m_{\triangle}: \mathfrak{M}_{0, S} \longrightarrow \mathfrak{M}_{0, S_{1}} \times \mathfrak{M}_{0, S_{2}},
$$

where $S_{1}=\left\{s_{1}, s_{2}, \ldots, s_{m+3}\right\}$ and $S_{2}=\left\{s_{1}, s_{2}, s_{3}, s_{m+4}, \ldots, s_{n}\right\}$. We deduce that

$$
\int_{X_{S_{1}, \delta_{1}}} \Omega(\underline{\epsilon}) \int_{X_{S_{2}, \delta_{2}}} \Omega\left(\underline{\epsilon}^{\prime}\right)=\sum_{\gamma \in G_{m_{\triangle}}} \int_{X_{S, \gamma}} m_{\triangle}^{*}\left(\Omega(\underline{\epsilon}) \otimes \Omega\left(\underline{\epsilon}^{\prime}\right)\right) .
$$

Recall from $\S 2.7$ that $G_{m_{\triangle}}$ is the set of $(m, \ell-m)$-shuffles, and that, in simplicial coordinates, $X_{S, \delta}$ is the unit simplex. We therefore deduce that

$$
\begin{gathered}
\int_{0<t_{1}<\cdots<t_{m}<1} \bigwedge_{i=1}^{m} \frac{d t_{i}}{\epsilon_{i}-t_{i}} \times \int_{0<t_{m+1}<\cdots<t_{\ell}<1} \bigwedge_{i=m+1}^{\ell} \frac{d t_{i}}{\epsilon_{i}-t_{i}} \\
=\sum_{\sigma \in \mathfrak{S}(m, \ell-m)} \int_{0<t_{\sigma(1)}<\cdots<t_{\sigma(\ell)}<1} \bigwedge_{i=1}^{\ell} \frac{d t_{i}}{\epsilon_{i}-t_{i}},
\end{gathered}
$$

which, by (7.19), gives the shuffle product formula:

$$
\zeta(w) \zeta\left(w^{\prime}\right)=\sum_{\sigma \in \mathfrak{S}(m, \ell-m)} \zeta\left(x_{\sigma\left(\epsilon_{\ell}\right)} x_{\sigma\left(\epsilon_{\ell-1}\right)} \ldots x_{\sigma\left(\epsilon_{1}\right)}\right)=\zeta\left(w \omega w^{\prime}\right) .
$$

Now let us see what happens in the case of the cubical product map (2.54):

$$
m_{\square}: \mathfrak{M}_{0, S} \longrightarrow \mathfrak{M}_{0, S_{1}} \times \mathfrak{M}_{0, S_{2}},
$$

where $S_{1}=\left\{s_{2}, s_{3}, \ldots, s_{m+4}\right\}$ and $S_{2}=\left\{s_{m+4}, \ldots, s_{n}, s_{1}, s_{2}, s_{3}\right\}$. We deduce that

$$
\int_{X_{S_{1}, \delta_{1}}} \Omega(\underline{\epsilon}) \int_{X_{S_{2}, \delta_{2}}} \Omega\left(\underline{\epsilon}^{\prime}\right)=\int_{X_{S, \delta}} m_{\square}^{*}\left(\Omega(\underline{\epsilon}) \otimes \Omega\left(\underline{\epsilon}^{\prime}\right)\right),
$$

since in this case $G_{m_{\square}}$ is the single element $\{\delta\}$. In cubical coordinates, each fundamental cell is a hypercube, and thus we obtain the formula:

$$
\int_{[0,1]^{m}} \Omega_{c}(\underline{\epsilon}) \int_{[0,1]^{\ell-m}} \Omega_{c}\left(\underline{\epsilon}^{\prime}\right)=\int_{[0,1]^{\ell}} \Omega_{c}(\underline{\epsilon}) \Omega_{c}\left(\underline{\epsilon}^{\prime}\right)
$$


where

$$
\Omega_{c}\left(\epsilon_{1}, \ldots, \epsilon_{m}\right)=\bigwedge_{i=1}^{m} \frac{d\left(x_{i} \ldots x_{\ell}\right)}{\epsilon_{i}-x_{i} \ldots x_{\ell}} .
$$

One can write the product $\Omega_{c}(\underline{\epsilon}) \Omega_{c}\left(\underline{\epsilon}^{\prime}\right)$ as a sum of terms $\Omega_{c}\left(\underline{\epsilon}^{\prime \prime}\right)$ either using an identity due to Cartier (see [55]) or using a power series expansion due to Goncharov ([24], Lemma 9.6). We use the latter approach. Let $\eta_{i}=(1,0, \ldots, 0)$ denote a 1 followed by a sequence $i-1$ zeros. Then

$$
\Omega_{c}\left(\epsilon_{1}, \ldots, \epsilon_{m}\right)=\Omega_{c}\left(\eta_{n_{1}}, \ldots, \eta_{n_{r}}\right)=\sum_{0 \leq k_{1}<\cdots<k_{r}} y_{1}^{k_{1}} \ldots y_{r}^{k_{r}} d x_{1} \ldots d x_{m}
$$

where $y_{1}=x_{1} \ldots x_{n_{1}}, y_{2}=x_{n_{1}+1} \ldots x_{n_{2}}, \ldots, y_{r}=x_{n_{r}+1} \ldots x_{m}$. Expanding $\Omega_{c}\left(\underline{\epsilon}^{\prime}\right)$ in a similar way, we obtain

$$
\begin{aligned}
\Omega_{c}(\underline{\epsilon}) \Omega_{c}\left(\underline{\epsilon}^{\prime}\right) & =\sum_{0 \leq k_{1}<\cdots<k_{r}} y_{1}^{k_{1}} \ldots y_{r}^{k_{r}} \sum_{0 \leq k_{r+1}<\cdots<k_{t}} y_{r+1}^{k_{r+1}} \ldots y_{t}^{k_{t}} d x_{1} \ldots d x_{\ell} \\
& =\sum_{\sigma \in \bar{\Sigma}_{m, \ell-m}} \sum_{\sigma_{*}\left(k_{1}, \ldots, k_{t}\right)} y_{1}^{k_{1}} \ldots y_{t}^{k_{t}} d x_{1} \ldots d x_{\ell}=\sum_{\sigma \in \bar{\Sigma}_{m, \ell-m}} \Omega_{c}\left(\sigma\left(\underline{\epsilon}, \underline{\epsilon}^{\prime}\right)\right),
\end{aligned}
$$

where $\bar{\Sigma}_{m, \ell-m}$ is the set of stuffles in the stuffle product for quasi-symmetric power series [35], and $\sigma_{*}\left(k_{1}, \ldots, k_{t}\right)$ is the corresponding domain of summation. Substituting this into (7.24), we deduce the stuffle product formula (see [51]):

$$
\zeta(w) \zeta\left(w^{\prime}\right)=\zeta\left(w \star w^{\prime}\right)
$$

Note, however, that the variables $x_{1}, \ldots, x_{\ell}$ in the expressions $\Omega_{c}\left(\sigma\left(\underline{\epsilon}, \underline{\epsilon^{\prime}}\right)\right)$ above can appear in the 'wrong order'. Thus (7.26) is proved after allowing permutations of the variables $x_{1}, \ldots, x_{\ell}$, which clearly preserves the unit cube $[0,1]^{r}$, and does not affect the integrals. The map which permutes the set of cubical coordinates $x_{1}, \ldots, x_{\ell}$, however, does not preserve the boundary components of the moduli space $\mathfrak{M}_{0, n}$ starting from $n \geq 6$.

REMARK 7.12. - This approach can be used to derive any number of elementary products between multiple zeta values. To make such a product explicit, one needs to fix a rule for decomposing a product of $\ell$-forms into a sum of $\ell$-forms of a preferred type (for example, $\Omega(\underline{\varepsilon})$ or $\Omega_{c}(\underline{\varepsilon})$ in the above examples). The motivic origin of such a product formula follows immediately from Remark 7.11 above (note that this does not quite suffice to prove the motivic nature of the classical stuffle product (7.26) due to complications coming from the permutation of cubical coordinates). We see by looking at the sets $G_{m_{\square}}$ and $G_{m_{\triangle}}$, that the shuffle and stuffle product formulae are extreme cases of a range of intermediary product formulae, obtained by shuffling together two subsets of $\left\{s_{3}, \ldots, s_{\ell}\right\}$ relative to $s_{1}=1, s_{2}=\infty$, and $s_{3}=0$. Such modular products will be studied elsewhere. 


\subsection{Product formulae for integrals of generalised polylogarithms}

More generally, we can apply the product formulae to convergent iterated integrals of functions of arbitrary weight, rather than just regular algebraic forms. If $f$ is a nondegenerate coordinate system $(\S 2.10)$, there is a commutative diagram

$$
\begin{array}{ccc}
f^{*}: \bigotimes_{i=1}^{k} B\left(\mathfrak{M}_{0, T_{i}}\right) & \longrightarrow & B\left(\mathfrak{M}_{0, S}\right) \\
\downarrow l & \downarrow l \\
\bigotimes_{i=1}^{k} L^{v_{i}, \delta_{i}}\left(\mathfrak{M}_{0, T_{i}}\right) & \longrightarrow L^{v, \delta}\left(\mathfrak{M}_{0, S}\right),
\end{array}
$$

where $f^{*}$ is a map of differential graded algebras. The vertices $v, v_{1}, \ldots, v_{k}$ are chosen such that $f(v)=\left(v_{1}, \ldots, v_{k}\right) \in \prod_{i=1}^{k} \mathfrak{M}_{0, T_{i}}^{\delta_{i}}$. The vertical maps are given by canonical regularisation maps $\rho_{v, \delta}, \rho_{v_{i}, \delta_{i}}$ defined in $\S 6$.7. Since each $B\left(\mathfrak{M}_{0, T_{i}}\right)$ is differentially simple, and since the map $f_{T_{i}}^{*}$ is non-zero for each $1 \leq i \leq k$, it follows that $f^{*}$ is injective. The horizontal map along the bottom is given by composition and multiplication of functions:

$$
\left(p_{1}, \ldots, p_{k}\right) \mapsto p_{1} \circ f_{T_{1}} \times \cdots \times p_{k} \circ f_{T_{k}} .
$$

In the same way as $\S 7.5$, we deduce the product formula:

$$
\prod_{i=1}^{k} \int_{\bar{X}_{T_{i}, \delta_{i}}} p_{i}=\sum_{\gamma \in G_{f}} \int_{X_{S, \gamma}} \prod_{i=1}^{k} p_{i} \circ f_{T_{i}},
$$

where we suppose that all integrals are convergent. In this way, we can obtain product formulae for generalised period integrals (integrals of polylogarithms).

In $\S 6.6$ we defined an action of the dihedral group of symmetries on the space of functions $L_{\mathcal{Z}}^{\delta}\left(\mathfrak{M}_{0, S}\right)$. We therefore have the following formula for any function $f \in L^{v, \delta}\left(\mathfrak{M}_{0, S}\right)$ such that the integral converges:

$$
\int_{\bar{X}_{S, \delta}} f=\int_{\bar{X}_{S, \delta}} \sigma^{*} f \quad \text { for all } \sigma \in D_{2 n} .
$$

If we combine dihedral symmetries with such product formulae, we have much freedom for manipulating integrals of generalised polylogarithms over $\bar{X}_{S, \delta}$. In particular, we can replace a period integral over any given face of the Stasheff polytope $\bar{X}_{S, \delta}$ with one over a face of fixed combinatorial type.

Lemma 7.13. - Let $F_{0} \subset \partial \bar{X}_{S, \delta}$ denote a fixed face of the Stasheff polytope $\bar{X}_{S, \delta}$ which corresponds to a short chord $\{i, i+1\}$ in the $n$-gon $(S, \delta)$. Given any other face $F \subset \partial \bar{X}_{S, \delta}$, and any form $\omega \in \Omega^{\ell-1} L_{\mathcal{Z}}^{\left.\delta\right|_{F}}(F)$, there exists another form $\omega^{\prime} \in \Omega^{\ell-1} L_{\mathcal{Z}}^{\left.\delta\right|_{F_{0}}}\left(F_{0}\right)$ such that

$$
\int_{F} \omega=\int_{F_{0}} \omega^{\prime}
$$

where the weight of $\omega$ is less than or equal to the weight of $\omega^{\prime}$.

Proof. - By using a product (7.27) we can replace the integral of $\omega$ over $F \cong \bar{X}_{k, \delta_{1}} \times \bar{X}_{n-1-k, \delta_{2}}$ with an integral over a face of $\partial \bar{X}_{S, \delta}$ of combinatorial type $\bar{X}_{n-1, \delta^{\prime}}$. Since the group of dihedral symmetries $D_{2 n}$ acts transitively on the set of all such faces, we can replace this with an integral over the face $F_{0}$ by applying (7.28).

$4{ }^{\mathrm{e}}$ SÉRIE - TOME $42-2009-\mathrm{N}^{\mathrm{o}} 3$ 


\subsection{Examples of period integrals in small dimensions}

First of all, consider the case $\mathfrak{M}_{0, S}^{\delta}(\mathbb{R})$, where $S=\left\{s_{1}, \ldots, s_{5}\right\}$, with the obvious dihedral structure which we denote $\delta$. We shall work in cubical coordinates $\left(x_{1}, x_{2}\right)$, which we write $(x, y)$. The set of chords $\chi_{S, \delta}$ is $\{13,24,35,41,52\}$, and the dihedral coordinates are

$$
u_{13}=1-x y, \quad u_{24}=x, \quad u_{35}=\frac{1-x}{1-x y}, \quad u_{41}=\frac{1-y}{1-x y}, \quad u_{52}=y .
$$

The domain $\bar{X}_{S, \delta}$ is bounded by the five sets of equations $u_{i j}=0, u_{i+1 j+1}=u_{i-1 j-1}=1$ for each pair $\{i, j\} \in \chi_{S, \delta}$, and these form the sides of a pentagon whose interior is $X_{S, \delta}$. In all, there are ten stable partitions of the set $\left\{s_{1}, \ldots, s_{5}\right\}$, which means that there are another five divisors at infinity given by the five equations $u_{i j}=\infty, u_{i-2 j-2}=u_{i+2} j+2=0$, where $\{i, j\} \in S$. These too form a pentagon.

The volume form $\omega_{S, \delta}=d \log u_{13} \wedge d \log u_{24}=(1-x y)^{-1} d x d y$, and every period integral on $\bar{X}_{S, \delta}$ is a sum of integrals

$$
I_{\chi_{S, \delta}}\left(\alpha_{i j}\right)=\int_{X_{S, \delta}} u_{13}^{\alpha_{13}} u_{24}^{\alpha_{24}} u_{35}^{\alpha_{35}} u_{41}^{\alpha_{41}} u_{52}^{\alpha_{52}} \omega_{S, \delta}
$$

which in cubical coordinates is just

$$
I_{5}(h, i, j, k, l)=\int_{0}^{1} \int_{0}^{1} \frac{x^{h}(1-x)^{i} y^{k}(1-y)^{j}}{(1-x y)^{i+j-l}} \frac{d x d y}{1-x y},
$$

where we have set $\alpha_{24}=h, \alpha_{35}=i, \alpha_{41}=j, \alpha_{52}=k, \alpha_{13}=l$. This exactly coincides with the family of integrals first defined by Dixon, and studied by Rhin and Viola [47, 17]. The dihedral group $D_{10}$ preserves the integral and permutes the indices $\{h, i, j, k, l\}$. It is generated by a cyclic rotation of order five $\tau_{5}$, and a reflection of order two $\sigma_{5}$, where

$$
\tau_{5}(x, y)=\left(1-x y, \frac{1-y}{1-x y}\right), \quad \sigma_{5}(x, y)=(y, x) .
$$

REMARK 7.14. - By combining the action of the dihedral symmetry group $D_{12}$ on $\mathfrak{M}_{0,6}$ and the product formula for integrals on $\mathfrak{M}_{0,4} \times \mathfrak{M}_{0,5}$ one can deduce the "hypergeometric transformation formula' for the integrals above. This remarkable identity was discovered by Dixon in 1905, and was exploited by Rhin and Viola to obtain the best irrationality measures for $\zeta(2)$ known to date. It is:

$$
\frac{1}{j ! k !} I(h, i, j, k, l)=\frac{1}{(k+l-i) !(i+j-l) !} I(h, i, k+l-i, i+j-l, l) .
$$

Before proving this identity, first observe that the real period integral $I_{4}$ on $\mathfrak{M}_{0,4}$ is the following beta integral:

$$
I_{4}\left(\alpha_{13}, \alpha_{24}\right)=\int_{0}^{1} x^{\alpha_{24}}(1-x)^{\alpha_{13}} d x=\frac{\alpha_{13} ! \alpha_{24} !}{\left(\alpha_{13}+\alpha_{24}+1\right) !} \in \mathbb{Q} .
$$

Now consider the case $\mathfrak{M}_{0,6}$. In cubical coordinates $(x, y, z)=\left(x_{1}, x_{2}, x_{3}\right)$ we have:

$$
\begin{gathered}
u_{13}=1-x y z, u_{24}=x, u_{35}=\frac{1-x}{1-x y}, u_{46}=\frac{(1-x y z)(1-y)}{(1-x y)(1-y z)}, u_{51}=\frac{1-z}{1-y z}, u_{62}=z, \\
u_{14}=\frac{1-y z}{1-x y z}, \quad u_{25}=y, \quad u_{36}=\frac{1-x y}{1-x y z} .
\end{gathered}
$$


Let $h, i, j, k, l, m, r, s, t$ be any nine non-negative integers. Let $I_{6}(h, i, j, k, l, m ; r, s, t)$ denote the period integral of weight three on $\mathfrak{M}_{0,6}$ which is therefore given by

$$
\int_{0}^{1} \int_{0}^{1} \int_{0}^{1} \frac{x^{h}(1-x)^{i} y^{t}(1-y)^{j} z^{l}(1-z)^{k}}{(1-x y)^{i+j-r}(1-y z)^{j+k-s}(1-x y z)^{r+s-j-m}} \frac{d x d y d z}{(1-x y)(1-y z)} .
$$

The dihedral group of symmetries $D_{12}$ for $\mathfrak{M}_{0,6}$ is generated by a cyclic permutation we denote $\tau_{6}=(h i j k l m)(r s t)$, and the reflection $\sigma_{6}=(h l)(i k)(r s)$. In the degenerate case where $r+s=j+m$ and $r=i+j+1$, the terms $(1-x y)$ and $(1-x y z)$ vanish in the integrand and (7.33) splits as a product $I_{4}(h, i) I_{5}(l, k, j, t, s)$. This is precisely a cubical product. Similarly, if the terms $(1-y z)$ and $(1-x y z)$ vanish, then we obtain a different splitting of the integral.

Proof of (7.30). Let $h, i, j, k, l$ be non-negative integers. We can assume without loss of generality that $i<l$. Set $\alpha=k+l-i$, and $\beta=i-l-1$. Consider

$$
I_{4}(\alpha, \beta) I_{5}(h, i, j, k, l)=I_{6}(h, i, j, \beta, \alpha, l+\beta+1 ; l, j+\beta+1, k) .
$$

We apply the cyclic permutation $\tau_{6}^{4}$ and use the fact that $\alpha+\beta+1=k, \alpha+i=k+l$ to obtain a different splitting. This gives

$$
I_{6}(j, \beta, \alpha, l+\beta+1, h, i ; k, l, j+\beta+1)=I_{4}(j, \beta) I_{5}(h, l+\beta+1, \alpha, j+\beta+1, l) .
$$

Replacing the $I_{4}$ terms with factorials using (7.31), we obtain the identity (7.30).

These examples illustrate how many important identities between multiple zeta values and Euler integrals can be proved by simple geometric considerations on moduli spaces $\mathfrak{M}_{0, n}$. Kontsevich and Zagier have made the very general and ambitious conjecture that every identity between periods can be proved using three elementary operations on integrals: changes of variables, the linearity of integration, and Stokes' theorem. In our situation, we have an infinite family of period integrals, but we have a fixed set of algebraic operations which we can perform on these integrals (e.g., the action of dihedral symmetries and the multiplication rules we defined above). It would be interesting to see which of the many known identities between multiple zeta values can be proved by using just these operations.

\section{Calculation of the periods of $\mathfrak{M}_{0, n}$}

We prove that the integral of a convergent algebraic $\ell$-form over an associahedron $X_{S, \delta}$ can be written as a linear combination of multiple zeta values of weight at most $\ell$. The key to the argument is the interplay between logarithmic singularities (which are permitted), and polar singularities (which are forbidden), along the boundaries of the associahedron $\bar{X}_{S, \delta}$.

\subsection{Pole-free primitives}

In order to apply Stokes' theorem to the manifold with corners $\bar{X}_{S, \delta}$, we need to verify that the algebra of generalised polylogarithms on $\mathfrak{M}_{0, S}^{\delta}$ satisfies the required properties. 
First of all, it follows from the regularization results of $\S 6.7$ that the coefficients of the generating series of generalized polylogarithms $L_{v, \delta}(z)$ have at most logarithmic singularities along the boundary of the Stasheff polytope $\bar{X}_{S, \delta}$. This implies that

$$
L_{\mathcal{Z}}^{\delta}\left(\mathfrak{M}_{0, S}\right) \subset \Gamma\left(\bar{X}_{S, \delta}, \mathcal{F}_{p}^{\log }\right) .
$$

Theorem 6.26 tells us that primitives exist in $L_{\mathcal{Z}}^{\delta}\left(\mathfrak{M}_{0, S}\right)$. One difficulty, however, is that primitives of $n$-forms on a manifold of dimension $n$ are not unique, and we may inadvertently introduce extra poles, which would give rise to divergent integrals. We show how to remove these extra poles below. In order to do this, we define

$$
L_{\mathcal{Z}}^{\delta,+}\left(\mathfrak{M}_{0, S}\right)=L_{\mathcal{Z}}^{\delta}\left(\mathfrak{M}_{0, S}\right) \cap \Gamma\left(\bar{X}_{S, \delta}, \mathcal{F}^{\log }\right)
$$

to be the sub-algebra of polylogarithms on $\mathfrak{M}_{0, S}$ which have at most logarithmic singularities on the boundary faces of the Stasheff polytope $\bar{X}_{S, \delta}$. Observe that every generalised polylogarithm has a canonical branch on $X_{S, \delta}$, and is therefore a well-defined, real-valued function.

Proposition 8.1. - Let $f \in W^{k} \Omega^{\ell} L_{\mathcal{Z}}^{\delta,+}\left(\mathfrak{M}_{0, S}\right)$. There exists a pole-free primitive

$$
F \in W^{k+1}\left(\Omega^{\ell-1} L_{\mathcal{Z}}^{\delta,+}\left(\mathfrak{M}_{0, S}\right)\right)
$$

such that $d F=f$, which implies that the restriction of $F$ to $\partial \bar{X}_{S, \delta}$ is continuous. In other words, the conditions of Theorem 4.11 hold.

Proof. - We know from Theorem 6.26 that $L_{\mathcal{Z}}^{\delta}\left(\mathfrak{M}_{0, S}\right)$ has trivial de Rham cohomology. It follows that we can find a primitive $G \in \Omega^{\ell-1} L_{\mathcal{Z}}^{\delta}\left(\mathfrak{M}_{0, S}\right)$ for $f$, of weight at most $k+1$, which may have polar singularities along $\partial \bar{X}_{S, \delta}$.

In order to remove spurious poles in $G$, we work on a single chart of $\bar{X}_{S, \delta}$ at a time. Therefore, let $e \in \chi_{S, \delta}^{q}$ denote a partial decomposition of the $n$-gon $(S, \delta)$, and let $\alpha \in \chi_{S, \delta}^{\ell}$ be a full triangulation which contains $e$. For every small $\varepsilon>0$, recall that there is a chart $U_{e}(\varepsilon)$ (see (4.2)), which has local (vertex) coordinates $x_{1}^{\alpha}, \ldots, x_{\ell}^{\alpha}$, which are canonical up to permutations. Recall that $U_{e}(\varepsilon) \cong U_{p, q}$ where $p+q=\ell(\S 4)$. It contains the face $F_{e}=\left\{u_{i j}=0:\{i, j\} \in e\right\}$, and we can assume, by reordering the coordinates if necessary, that $F_{e}=\left\{x_{1}^{\alpha}=\cdots=x_{q}^{\alpha}=0\right\}$. We remove polar singularities with respect to each coordinate $x_{1}^{\alpha}, \ldots, x_{q}^{\alpha}$ in turn. First, there is a decomposition $G=G_{p}+G^{\prime}$, where $G^{\prime}$ has at most logarithmic singularities in $x_{1}^{\alpha}$, and $G_{p}$ is the divergent part of $G$ along $\left\{x_{1}^{\alpha}=0\right\}$ :

$$
G_{p}=\sum_{a \geq 0, b \geq 1} \frac{\log ^{a} x_{1}^{\alpha}}{\left(x_{1}^{\alpha}\right)^{b}} g_{a, b}\left(x_{2}^{\alpha}, \ldots, x_{\ell}^{\alpha}\right) \omega_{a, b}+\sum_{c \geq 1} \log ^{c} x_{1}^{\alpha} h_{c}\left(x_{2}^{\alpha}, \ldots, x_{\ell}^{\alpha}\right) d x_{2}^{\alpha} \ldots d x_{\ell}^{\alpha},
$$

where $g_{a, b}\left(x_{2}^{\alpha}, \ldots, x_{\ell}^{\alpha}\right), h_{c}\left(x_{2}^{\alpha}, \ldots, x_{\ell}^{\alpha}\right) \in \mathcal{F}_{p}^{\log }\left(U_{p, q-1}\right)$, and where $\omega_{a, b}$ are any $\ell-1$ forms $\sum_{i} a_{i} d x_{1}^{\alpha} \ldots \widehat{d x_{i}^{\alpha}} \ldots d x_{\ell}^{\alpha}$, where $a_{i} \in \mathbb{R}$. By differentiating this expression, and using the fact that $d G=f$ has no poles, it is easy to verify that $d G_{p}=0$ (in other words, poles can only get worse on differentiating). Therefore $d G^{\prime}=f$, and so $G^{\prime}$ is a primitive of $f$ which has no poles along $x_{1}^{\alpha}=0$. Using the fact that $L_{\mathcal{Z}}^{\delta}\left(\mathfrak{M}_{0, S}\right)$ is closed under differentiation with respect to $x_{i}^{\alpha}$, and closed under taking regularised limits at $x_{i}^{\alpha}=0$, for $1 \leq i \leq \ell$ (Theorem 6.25), one can easily check that $G^{\prime}$ lies in $L_{\mathcal{Z}}^{\delta}\left(\mathfrak{M}_{0, S}\right)$, i.e., $G^{\prime}$ is still a generalised polylogarithm. Repeating this argument for $x_{1}^{\alpha}, \ldots, x_{q}^{\alpha}$, in turn, we obtain a primitive of $f$ with no poles on the local chart $U_{\alpha}(\varepsilon)$. The whole argument can then be repeated on each local chart of $X_{S, \delta}$, 
and we end up with a primitive $F$ of $f$ which has no poles anywhere along $\partial \bar{X}_{S, \delta}$. This is because, whenever we remove a polar singularity along the divisor $D_{i j},\{i, j\} \in \chi_{S, \delta}$, no new poles are created along any other boundary component $D_{k l}$, where $\{k, l\} \in \chi_{S, \delta}$, and the total weight is not increased. This proves the proposition. The fact that the restriction of $F$ to each component of the boundary is continuous follows from Lemma 4.10.

In $\S 8.3$ we show how to construct canonical primitives which are automatically free of poles along $\partial \bar{X}_{S, \delta}$.

\subsection{Proof of the main theorem}

Let $S$ denote a set of order $n=\ell+3$ with a fixed dihedral structure $\delta$.

THEOREM 8.2. - For all sets of indices $\alpha_{i j} \geq 0$,

$$
I_{S, \delta}\left(\alpha_{i j}\right)=\int_{\bar{X}_{S, \delta}} \prod_{\{i, j\} \in \chi_{S, \delta}} u_{i j}^{\alpha_{i j}} \omega_{S, \delta} \in W^{\ell} \mathcal{Z} .
$$

Proof. - The proof is by induction and by repeated application of Stokes' theorem (Theorem 4.11). We write $S=S_{n}=\left\{s_{1}, \ldots, s_{n}\right\}$. First observe that the regular $\ell$-form

$$
f_{0}=\prod_{\{i, j\} \in \chi_{S, \delta}} u_{i j}^{\alpha_{i j}} \omega_{S, \delta} \in W^{0}\left(\Omega^{\ell} L_{\mathcal{Z}}^{\delta}\left(\mathfrak{M}_{0, S}\right)\right)
$$

has no poles on the compact set $\bar{X}_{S, \delta}$. Let us write

$$
S_{i}=\left\{s_{1}, \ldots, s_{i}\right\} \quad \text { for all } \quad 3 \leq i \leq n,
$$

and let $\delta_{b}$ denote the dihedral structure on $S_{b} \subset S$ induced by $\delta$. This is equivalent to choosing a nested sequence of sub-faces of $\bar{X}_{S, \delta}$ in its stratification. Let $0 \leq b \leq \ell$, and suppose by induction that there exists an $\ell-b$ form

$$
f_{b} \in W^{b}\left(\Omega^{\ell-b} L_{\mathcal{Z}}^{\delta,+}\left(\mathfrak{M}_{0, S_{n-b}}\right)\right),
$$

which has no poles on $\bar{X}_{S_{n-b}, \delta_{n-b}}$, such that

$$
I_{S, \delta}\left(\alpha_{i j}\right)=\int_{\bar{X}_{S_{n-b}, \delta_{n-b}}} f_{b} .
$$

By Proposition 8.1, there exists a primitive $P \in \Omega^{\ell-b-1} L_{\mathcal{Z}}^{\delta,+}\left(\mathfrak{M}_{0, S_{n-b}}\right)$ of weight at most $b+1$, which has no poles in $\bar{X}_{S_{n-b}, \delta_{n-b}}$, and is continuous on the interior of $\partial \bar{X}_{S_{n-b}, \delta_{n-b}}$. By the version of Stokes' formula stated in Theorem 4.11,

$$
I_{S, \delta}\left(\alpha_{i j}\right)=\int_{\bar{X}_{S_{n-b}, \delta_{n-b}}} f_{b}=\int_{\partial \bar{X}_{S_{n-b}, \delta_{n-b}}} P .
$$

By the geometry of the Stasheff polytopes ( $(2.2)$, we know that

$$
\partial \bar{X}_{S_{n-b}, \delta_{n-b}}=\bigcup_{\{i, j\} \in \chi_{S_{n-b}, \delta_{n-b}}} F_{i j}
$$

where $F_{i j}=F_{i j}\left(\bar{X}_{S_{n-b}, \delta_{n-b}}\right)$ is the face corresponding to the chord $\{i, j\}$, and therefore

$$
I_{S, \delta}\left(\alpha_{i j}\right)=\left.\sum_{\{i, j\} \in \chi_{S_{n-b}, \delta_{n-b}}} \int_{F_{i j}} P\right|_{F_{i j}} .
$$


Given a chord $\{i, j\} \in \chi_{S_{n-b}, \delta_{n-b}}$, there exists a partition of $S_{n-b}=T_{1} \cup T_{2}$ such that $F_{i j} \cong \bar{X}_{T_{1} \cup\{e\}, \delta_{1}} \times \bar{X}_{T_{2} \cup\{e\}, \delta_{2}}$, where $e$ corresponds to the chord $\{i, j\}$ (Equation (2.39)) and $\delta_{1}, \delta_{2}$ are the induced dihedral structures. By Theorem 6.25, we have

$$
\left.P\right|_{F_{i j}} \in W^{b+1} \Omega^{\ell-b-1}\left(L_{\mathcal{Z}}^{\delta_{1}}\left(\mathfrak{M}_{0, T_{1} \cup\{e\}}\right) \otimes L_{\mathcal{Z}}^{\delta_{2}}\left(\mathfrak{M}_{0, T_{2} \cup\{e\}}\right)\right) .
$$

By Lemma 7.13 , there exists $g_{i j} \in W^{b+1}\left(\Omega^{\ell-b-1} L_{\mathcal{Z}}^{\delta}\left(\mathfrak{M}_{0, S_{n-b-1}}\right)\right)$ such that

$$
\left.\int_{\bar{X}_{T_{1} \cup\{e\}, \delta_{1}} \times \bar{X}_{T_{2} \cup\{e\}, \delta_{2}}} P\right|_{F_{i j}}=\int_{\bar{X}_{S_{n-b-1}, \delta_{n-b-1}}} g_{i j} .
$$

Thus each integral in the sum (8.3) can be written as an integral over the fixed face $\bar{X}_{S_{n-b-1}, \delta_{n-b-1}}$ by applying product formulae and using dihedral symmetries. Since $\left.P\right|_{F_{i j}}$ is continuous with at most logarithmic singularities along $\partial F_{i j}$, it follows that $g_{i j} \in \Omega^{\ell-b-1}\left(L_{\mathcal{Z}}^{\delta,+}\left(\mathfrak{M}_{0, S_{n-b-1}}\right)\right)$. Taking the sum over all $\{i, j\} \in \chi_{S_{n-b-1}, \delta_{n-b-1}}$ in (8.3), we obtain a form $f_{b+1} \in W^{b+1}\left(\Omega^{\ell-b-1} L_{\mathcal{Z}}^{\delta,+}\left(\mathfrak{M}_{0, S_{n-b-1}}\right)\right)$ such that

$$
I_{S, \delta}\left(\alpha_{i j}\right)=\int_{\bar{X}_{S_{n-b-1}, \delta_{n-b-1}}} f_{b+1} .
$$

This completes the induction step. At the final stage of the induction, we deduce that $I_{S, \delta}$ is given by evaluating a multiple polylogarithm in one variable in $W^{\ell} L_{\mathcal{Z}}^{\delta}\left(\mathfrak{M}_{0,4}\right)$ at a single point. We conclude (see $\S 5.5)$ that $I_{S, \delta}\left(\alpha_{i j}\right) \in W^{\ell} \mathcal{Z}$.

Note that it is not strictly necessary in the course of the above proof to use the product formula (Lemma 7.13). This replaces the sum of a product of integrals with a single integral at each stage, and only serves to simplify notations. Lemma 7.4 implies the following result.

COROLlaRY 8.3. - Every relative period integral over a union of cells $X_{S, \delta_{i}}$ can be written as a linear combination of multiple zeta values of weight at most $\operatorname{dim} \mathfrak{M}_{0, S}(\mathbb{R})$.

\subsection{Canonical primitives - an algorithmic approach}

The existence of primitives uses the fact that $\mathfrak{M}_{0, S}$ is a fibre-type hyperplane arrangement. By exploiting the hyperlogarithm fibration, we can find canonical primitives, as in Remark 3.42 , which have no spurious poles. This gives rise to a simplified series of integrals occurring in the proof of Theorem 8.2 above, and yields an effective algorithm for computing period integrals on $\mathfrak{M}_{0, S}$ algebraically.

Let $f \in W^{b} \Omega^{\ell} L_{\mathcal{Z}}^{\delta,+}\left(\mathfrak{M}_{0, S}\right)$. Working in cubical coordinates, we can write $f=g\left(x_{1}, \ldots, x_{\ell}\right) d x_{1} \ldots d x_{\ell}$, where $g \in L_{\mathcal{Z}}^{\delta,+}\left(\mathfrak{M}_{0, S}\right)$ is of weight at most $b$. Recall that by Remark 3.42, there exists a primitive $F \in W^{b+1} \Omega^{\ell-1} L_{\mathcal{Z}}^{\delta}\left(\mathfrak{M}_{0, S}\right)$ such that

$$
F=G\left(x_{1}, \ldots, x_{\ell}\right) d x_{1} \ldots d x_{\ell-1}
$$

where $\partial G / \partial x_{\ell}=g$. More concretely, let $S=\left\{s_{1}, \ldots, s_{n}\right\}$ and let $S^{\prime}=\left\{s_{2}, \ldots, s_{n}\right\}$. Recall from $\S 6.7$ that the hyperlogarithm fibration given by projection onto $x_{\ell}=0$ :

$$
\mathfrak{M}_{0, S} \longrightarrow \mathfrak{M}_{0, S^{\prime}}
$$

gives rise to a decomposition of filtered algebras

$$
L_{\mathcal{Z}}^{\delta}\left(\mathfrak{M}_{0, S}\right) \cong L_{\mathfrak{M}_{0, S^{\prime}}}\left(\mathbb{P}^{1} \backslash \Sigma\right) \otimes_{\mathcal{O}\left(\mathfrak{M}_{0, S^{\prime}}\right)} L_{\mathcal{Z}}^{\delta^{\prime}}\left(\mathfrak{M}_{0, S^{\prime}}\right)
$$


where $\Sigma=\left\{0,1, x_{\ell-1}^{-1}, \ldots,\left(x_{1} \ldots x_{\ell-1}\right)^{-1}\right\}$ as in $\S 5.4$, and $\delta^{\prime}$ is the induced dihedral structure on $S^{\prime}$. We can therefore write the function $g$ as a finite sum of products

$$
g\left(x_{1}, \ldots, x_{\ell}\right)=\sum_{i} a_{i}\left(x_{\ell}\right) b_{i}\left(x_{1}, \ldots, x_{\ell-1}\right),
$$

where each $b_{i} \in L_{\mathcal{Z}}^{\delta^{\prime}}\left(\mathfrak{M}_{0, S^{\prime}}\right)$ is a function of $\ell-1$ variables $x_{1}, \ldots, x_{\ell-1}$ only, and each $a_{i}$, considered as a function of the single variable $x_{\ell}$, is a hyperlogarithm with singularities in $\Sigma \cup \infty$. We can assume that the $a_{i}$ are linearly independent. The weight of each product of $a_{i}\left(x_{\ell}\right)$ and $b_{i}\left(x_{1}, \ldots, x_{\ell-1}\right)$ is at most $b$. Now by Proposition 3.22, each function $a_{i}\left(x_{\ell}\right)$ has a primitive (with respect to the variable $x_{\ell}$ ) which we denote

$$
A_{i}\left(x_{\ell}\right) \in L_{\mathfrak{M}_{0, S^{\prime}}}\left(\mathbb{P}^{1} \backslash \Sigma\right)
$$

which is of weight at most one more than the weight of $a_{i}\left(x_{\ell}\right)$. We can choose the constant of integration in such a way that $A_{i}\left(x_{\ell}\right)$ either vanishes at 0 , or is $\log ^{k}\left(x_{\ell}\right)$ for some $k \geq 1$ (see $\S 5.2$ ). In the latter case, $a_{i}\left(x_{\ell}\right)$, and hence $g\left(x_{1}, \ldots, x_{\ell}\right)$ would have a pole at the origin, so this cannot occur (this is precisely the argument in the proof of Lemma 4.10). It follows that the function

$$
F=\sum_{i} A_{i}\left(x_{\ell}\right) b_{i}\left(x_{1}, \ldots, x_{\ell-1}\right) d x_{1} \ldots d x_{\ell-1}
$$

is a primitive of $f$, and is identically zero on all faces of $\bar{X}_{S, \delta}$ except the single face given by $x_{\ell}=1$. The primitive $F$ has no poles since this would contradict the convergence of the integral by Lemma 4.9. It is therefore continuous on the interior of this face, and we can apply Stokes' theorem directly. This approach to the induction step in the proof of the main theorem has the advantage that it does not involve any regularisation, or having to apply a product formula (Lemma 7.13).

\subsection{Taylor expansions of Selberg integrals and multi-beta functions}

The method of proof of Theorem 8.2 works much more generally, and enables us to compute integrals of arbitrary generalised polylogarithms on $\mathfrak{M}_{0, S}$, which are allowed logarithmic singularities along the boundary of the domain of integration.

Theorem 8.4. - Let $f \in W^{k} L_{\mathcal{Z}}^{\delta,+}\left(\mathfrak{M}_{0, S}\right)$ denote a generalised polylogarithm on $\mathfrak{M}_{0, S}$ of weight at most $k$, which has no poles along $\partial \bar{X}_{S, \delta}$. Then

$$
I(f)=\int_{\bar{X}_{S, \delta}} f \omega_{S, \delta} \in W^{\ell+k} \mathcal{Z} .
$$

The proof is identical to the proof of Theorem 8.2. Note that the integrand is always welldefined on the real domain $X_{S, \delta}$ at each stage of the induction. If we apply this theorem in the case where $f$ is an $\mathcal{O}\left(\mathfrak{M}_{0, S}\right)$-linear combination of products of powers of logarithms, then we deduce the following corollary.

COROLLARY 8.5. - Let $\left\{s_{i j}\right\}$ denote a set of complex parameters. It follows from the calculations in $\S 4$ that the following integral, viewed as a function of the variables $s_{i j}$, is holomorphic in the region $\operatorname{Re} s_{i j}>-1$ :

$$
\beta_{S, \delta}\left(\left\{s_{i j}\right\}\right)=\int_{\bar{X}_{S, \delta}} \prod_{\{i, j\} \in \chi, \delta} u_{i j}^{s_{i j}} \omega_{S, \delta} .
$$


The coefficients of its Taylor expansion (with respect to the variables $s_{i j}$ ) at any integral point $s_{i j} \in \mathbb{Z}$, where $s_{i j} \geq 0$ for all $\{i, j\} \in \chi_{S, \delta}$, are multiple zeta values.

Similar kinds of results have been obtained by Terasoma [49]. The integral (8.4) defines a multi-beta function, since in the case $\mathfrak{M}_{0,4}$ it reduces to the ordinary beta function. It satisfies many functional identities coming from the dihedral relations (2.10), the product maps (7.27), and also the action of the dihedral symmetry group, and would merit further study.

\subsection{Computation of relative periods of the moduli spaces $\mathfrak{M}_{0, S}$}

Let $A, B$ denote two sets of divisors in $\overline{\mathfrak{M}}_{0, S} \backslash \mathfrak{M}_{0, S}$ which do not share any irreducible components $(\S 7.2)$, and consider a period integral of the form (7.12), whose integrand is an algebraic $\ell$-form on $\overline{\mathfrak{M}}_{0, S} \backslash A$ defined over $\mathbb{Q}$. We sketch a proof of the following theorem in this case. Using hypercohomological methods, one should be able to deduce with a little extra work that all the periods of $H^{\ell}\left(\overline{\mathfrak{M}}_{0, S} \backslash A, B \backslash B \cap A\right)$, for any $A, B$, are expressible in terms of multiple zeta values and $2 i \pi$.

TheOREM 8.6. - Any such period of $H^{\ell}\left(\overline{\mathfrak{M}}_{0, S} \backslash A, B \backslash B \cap A\right)$ is a $\mathbb{Q}$-linear combination of multiple zeta values and the constant $2 i \pi$, of total weight at most $\ell$.

Let $\Delta_{B} \subset \mathfrak{M}_{0, S}(\mathbb{C})$ denote any real smooth compact submanifold with corners of dimension $\ell$, whose boundary is contained in the set of complex points of $B$. Let $\omega \in \Omega^{\ell}\left(\overline{\mathfrak{M}}_{0, S} \backslash A\right)$ be an algebraic $\ell$-form as above. We can assume that $\Delta_{B}$ is disjoint from $A$, and that $\Delta_{B}$ is stratified by $B$ according to (7.11). Let $A$ be a union of distinct divisors $A_{i}$, for $1 \leq i \leq N$.

By decomposing the relative homology class $\left[\Delta_{B}\right] \in H_{\ell}\left(\overline{\mathfrak{M}}_{0, S} \backslash A, B \backslash B \cap A\right)$ into different pieces, we can first consider the case when $\Delta_{B}$ does not wind non-trivially around any component $A_{i}$ of $A$. In this case, write $X=\Delta_{B}$, and observe that the argument given in $\S 8.2$ goes through as before. In other words, we can take primitives in the algebra of polylogarithms $L_{\mathcal{Z}}^{\delta}\left(\mathfrak{M}_{0, S}\right)$, and repeatedly apply Stokes' formula (Theorem 4.11) to the manifold with corners $X$, and proceed by induction. Note that, although $X$ is not necessarily simplyconnected, the argument goes through as long as the functions we integrate remain singlevalued along $X$. Since $X$ does not wind around $A$, we can ensure that this is the case. This proves that

$$
\int_{X} \omega \in W^{\ell} \mathcal{Z}
$$

In the case when $\Delta_{B}$ winds around some component of $A$, we apply a residue formula and induction. To make this precise, let $A_{i}^{c}=\bigcup_{j \neq i} A_{j}$ for all $1 \leq i \leq \ell$, and consider the residue map

$$
H^{\ell}\left(\overline{\mathfrak{M}}_{0, S} \backslash A, B \backslash B \cap A\right) \longrightarrow \bigoplus_{i=1}^{N} H^{\ell-1}\left(A_{i} \backslash\left(A_{i} \cap A_{i}^{c}\right),\left(B \cap A_{i}\right) \backslash\left(B \cap A_{i} \cap A_{i}^{c}\right)\right),
$$

and its dual map

$$
H_{\ell}\left(\overline{\mathfrak{M}}_{0, S} \backslash A, B \backslash B \cap A\right) \longleftarrow \bigoplus_{i=1}^{N} H_{\ell-1}\left(A_{i} \backslash\left(A_{i} \cap A_{i}^{c}\right),\left(B \cap A_{i}\right) \backslash\left(B \cap A_{i} \cap A_{i}^{c}\right)\right) .
$$

Suppose that $\left[\Delta_{B}\right] \in H_{\ell}\left(\overline{\mathfrak{M}}_{0, S} \backslash A, B \backslash B \cap A\right) \quad$ is the image of a class $[Y] \in H_{\ell-1}\left(A_{i} \backslash\left(A_{i} \cap A_{i}^{c}\right),\left(B \cap A_{i}\right) \backslash\left(B \cap A_{i} \cap A_{i}^{c}\right)\right)$, for some $1 \leq i \leq N$, where 
$Y \subset A_{i} \backslash\left(A_{i} \cap A_{i}^{c}\right)$ is a smooth compact submanifold with corners of dimension $\ell-1$, i.e., a tubular neighbourhood of $A_{i}$. By taking the residue along $A_{i}$, we get:

$$
\int_{\Delta_{B}} \omega=\left.2 i \pi \int_{Y} \operatorname{Res} \omega\right|_{A_{i}} .
$$

The corresponding period is therefore $2 \pi i$ times a period of $H_{\ell-1}\left(A_{i} \backslash\left(A_{i} \cap A_{i}^{c}\right)\right.$, $\left.\left(B \cap A_{i}\right) \backslash\left(B \cap A_{i} \cap A_{i}^{c}\right)\right)$. Since $A_{i}$ is itself isomorphic to a product of moduli spaces, we can repeat the argument inductively. We conclude that, in all cases,

$$
\int_{\Delta_{B}} \omega \in W^{\ell} \mathcal{Z}[2 i \pi]
$$

where $\mathcal{Z}[2 i \pi]$ has the natural filtration which gives $2 i \pi$ weight 1 .

\subsection{Some simple examples}

In the following examples, it is convenient to work in cubical coordinates $x_{1}, \ldots, x_{\ell}$. At each stage we take canonical primitives (as described in $\S 8.3$ ) with respect to $x_{1}$ or $x_{\ell}$. This is because the projection maps onto $x_{1}=0$ or $x_{\ell}=0$ are fibrations ( $(2.4)$, and so we can use the method of partial fractions to find primitives. At each stage, one can re-confirm (using dihedral coordinates) that the primitives have no poles along the boundary of the domain of integration $\bar{X}_{S, \delta}$. First, assume $|S|=5$, that is $S=\left\{s_{1}, \ldots, s_{5}\right\}$. We compute

$$
I_{1}=\int_{\bar{X}_{S, \delta}} \omega_{S, \delta}=\int_{0}^{1} \int_{0}^{1} \frac{d x d y}{1-x y} .
$$

Following $\S 8.3$, we take the primitive of $\omega_{S, \delta}$ with respect to the variable $y$. This is

$$
F=-\log (1-x y) \frac{d x}{x}
$$

which vanishes at $y=0$ as required. In dihedral coordinates (7.29), this is $F=-\log u_{13} d \log u_{24}$, which has no poles at finite distance. Then

$$
\int_{\bar{X}_{S, \delta}} \omega_{S, \delta}=\sum_{\{i, j\} \in \chi_{S, \delta}} \int_{D_{i j}}-\log u_{13} d \log u_{24} .
$$

The only face on which the form does not vanish is the face $D_{14}$ which is defined by $u_{14}=0$, $u_{25}=u_{35}=1$, which implies that $u_{13}=1-u_{24}$ by (2.10). We obtain

$$
\int_{\bar{X}_{S, \delta}} \omega_{S, \delta}=\int_{0}^{1}-\log (1-x) \frac{d x}{x} .
$$

Notice that the form $\log (1-x) d x / x$ is continuous on the interval $[0,1)$ but has a logarithmic singularity at $x=1$. It has a unique primitive which vanishes at 0 , namely $\operatorname{Li}_{2}(x)$, which is now bounded at $x=1$ by Lemma 4.10 . We conclude that

$$
I_{1}=\int_{\bar{X}_{S, \delta}} \omega_{S, \delta}=\left[\operatorname{Li}_{2}(x)\right]_{0}^{1}=\zeta(2)
$$

Now let $|S|=6$. Consider the following integral on $\mathfrak{M}_{0,6}$ :

$$
I_{2}=\int_{0 \leq t_{1} \leq t_{2} \leq t_{3} \leq 1} \frac{d t_{1}}{1-t_{1}} \frac{d t_{2}}{t_{2}} \frac{d t_{3}}{t_{3}-t_{1}}=\int_{[0,1]^{3}} \frac{d x d y d z}{(1-x y z)(1-x y)}=\int_{\bar{X}_{S, \delta}} u_{14} \omega_{S, \delta} .
$$


The last formula shows that $I$ converges. Working in cubical coordinates, we have

$$
I_{2}=\int_{[0,1]^{2}}\left[\frac{-\log (1-x y z)}{x y}\right]_{0}^{1} \frac{d x d y}{1-x y}=\int_{[0,1]^{2}} \frac{-\log (1-x y)}{x y} \frac{d x d y}{1-x y} .
$$

Using partial fractions with respect to the variable $y$,

$$
I_{2}=\int_{[0,1]^{2}} \frac{-\log (1-x y)}{x y}-\frac{\log (1-x y)}{1-x y} d x d y=\int_{0}^{1} \frac{1}{z}\left[\operatorname{Li}_{2}(x y)+\frac{1}{2} \log ^{2}(1-x y)\right]_{0}^{1} d x .
$$

We conclude that

$$
I_{2}=\int_{0}^{1} \frac{\operatorname{Li}_{2}(x)}{x}+\frac{\log ^{2}(1-x)}{2 x} d x=\left[\operatorname{Li}_{3}(x)+\operatorname{Li}_{1,2}(x)\right]_{0}^{1}=\zeta(3)+\zeta(1,2) .
$$

At each stage one can verify that the canonical primitives we have used above do not introduce any new poles along the boundary of the associahedron $\partial \bar{X}_{S, \delta}$. For example, the first primitive can be written using (7.32):

$$
\frac{-\log (1-x y z)}{x y} \frac{d x d y}{1-x y}=-\frac{\log \left(u_{13}\right)}{u_{24} u_{25}} \omega_{5, \delta}
$$

where $\omega_{5, \delta}=(1-x y)^{-1} d x d y$ is the pull-back of the canonical 2-form on $D_{26} \subset \mathfrak{M}_{0, S}^{\delta}$ along the map $(x, y, z) \mapsto(x, y): \mathfrak{M}_{0,6} \rightarrow \mathfrak{M}_{0,5}$. It has no poles at finite distance by definition (Lemma 7.1). Furthermore, we know by (2.10) that $1-u_{13}=u_{24} u_{25} u_{26}$, so (8.7) has no poles along $\bar{X}_{S, \delta}$ as required.

We give one final example for $\mathfrak{M}_{0,7}$, where the calculation is given entirely in cubical coordinates, and is still sufficiently simple to be checked by hand. Consider

$$
I_{3}=\int_{[0,1]^{4}} \frac{x(1-x) y(1-y) z(1-z) w(1-w)}{(1-x y z w)^{2}} d x d y d z d w
$$

After integration with respect to $w$, we have

$$
I_{3}=\int_{[0,1]^{3}} \frac{(1-x)(1-y)(1-z)}{(x y z)^{2}}\left[-2 x y z+(2-x y z) \operatorname{Li}_{1}(x y z)\right] d x d y d z .
$$

A further integration with respect to $z$ gives

$$
I_{3}=\int_{[0,1]^{2}} \frac{(1-x)(1-y)}{(x y)^{2}}\left[5 x y-(3-3 x y) \operatorname{Li}_{1}(x y)-(2+x y) \operatorname{Li}_{2}(x y)\right] d x d y .
$$

At the following stage, one obtains:

$$
I_{3}=\int_{[0,1]} \frac{(1-x)}{x^{2}}\left[-16 x+(9-9 x) \operatorname{Li}_{1}(x)+(5+4 x) \operatorname{Li}_{2}(x)+(2-x) \operatorname{Li}_{3}(x)\right] d x .
$$

Finally, this gives:

$$
I_{3}=55-30 \zeta(2)-2 \zeta(3)-3 \zeta(4)
$$

REMARK 8.7. - It would be interesting to determine the values of the simplest possible integrals

$$
\int_{X_{S, \delta}} \omega_{S, \delta}
$$

explicitly in terms of multiple zeta values, for all $n=|S|$. 


\section{Appendix}

Let $M$ be the complement of an affine hyperplane configuration, as defined in $\S 3$, and let $F$ denote its ring of regular functions. In the case where the de Rham cohomology ring $H^{\star}(F)$ only has quadratic relations, we can prove directly that all higher cohomology groups of $B(F)$ vanish.

THEOREM 9.1. - Let $F$ be the ring of regular functions on an affine hyperplane arrangement $M$ such that $H^{\star}(F)$ is a quadratic algebra. Then

$$
H_{\mathrm{DR}}^{0}(B(F))=k, \quad \text { and } \quad H_{\mathrm{DR}}^{i}(B(F))=0 \quad \text { for all } i \geq 1 \text {. }
$$

Proof. - Let $A \subset \Omega^{*}(F)$ denote the algebra generated by the 1-forms $\omega_{1}, \ldots, \omega_{N} \in H^{1}(F)$, where $\omega_{i}=d \log \alpha_{i}$ as defined in $\S 3.2$. Let $V_{1}(F)=\bigoplus_{i=1}^{N} k \omega_{i}$. Let $T$ denote the free tensor algebra over $V_{1}$. We can view $A$ as a quotient algebra of $T$ of the form $A=T / T Q T$, where $Q$ consists of finitely many quadratic relations $q_{1}, \ldots, q_{t}$ of the form

$$
q_{l}=\sum_{i, j} \lambda_{i j}^{l} \omega_{i} \otimes \omega_{j} \quad \text { for } 1 \leq l \leq t .
$$

Now recall that $\Omega^{\star}(B(F))=\Omega^{\star}(F) \otimes \bigoplus_{m \geq 0} V_{m}(F)$. The weight filtration on $\Omega^{\star}(B(F)$ ), defined by $W_{m} \Omega^{i} B(F)=\Omega^{i}(F) \otimes \bigoplus_{j=0}^{m} V_{j}(F)$, gives rise to a spectral sequence with $E_{0}$ terms

$$
E_{0}^{p, q}(B(F))=\Omega^{p+q}(F) \otimes V_{p}(F),
$$

which is bounded below and exhaustive, and therefore converges to the cohomology of $B(F)$. Consider the differential subalgebra $A(F)=A \otimes \bigoplus_{m \geq 0} V_{m}(F)$ of $\Omega^{\star}(B(F))$. It also defines a spectral sequence with $E_{1}$ terms

$$
E_{1}^{p, q}(A(F))=H^{p+q}(A) \otimes V_{p}(F) \cong H^{p+q}(F) \otimes V_{p}(F)=E_{1}^{p, q}(B(F)) .
$$

It follows that $H^{i}(B(F)) \cong H^{i}(A(F))$ for all $i \geq 0$, and it suffices to show that $H^{\star}(A(F))$ is trivial. Therefore consider an element

$$
f=\sum_{I=\left(i_{1}, \ldots, i_{m}\right)} \sum_{J=\left(j_{1}, \ldots, j_{n}\right)} \alpha_{I, J} \omega_{j_{1}} \wedge \cdots \wedge \omega_{j_{n}}\left[\omega_{i_{1}}|\ldots| \omega_{i_{m}}\right] \in A \otimes V_{m}(F),
$$

such that $d f=0$. This implies that

$$
\sum_{i_{1}, J} \alpha_{I, J} \omega_{j_{1}} \wedge \cdots \wedge \omega_{j_{n}} \wedge \omega_{i_{1}}=0 \quad \text { for all } i_{2}, \ldots, i_{m}
$$

Because $A$ is quadratic, this expression (viewed in the tensor algebra $T$ ) decomposes as a sum of relations of the form $w_{1} q^{l} w_{2}$, where $w_{1}, w_{2} \in T$. If $w_{2}$ is of degree $\neq 0$ in $T$, the corresponding relation is already zero in $f$. We deduce that $f$ is a sum of terms

$$
\sum_{I=\left(i_{2}, \ldots, i_{m}\right)} \sum_{l=1}^{t} \sum_{i, j} \lambda_{i j}^{l} \omega_{I, l} \wedge \omega_{i}\left[\omega_{j}\left|\omega_{i_{1}}\right| \ldots \mid \omega_{i_{m}}\right],
$$

where $\omega_{I, l} \in A$. Each such expression has a primitive

$$
\sum_{I=\left(i_{2}, \ldots, i_{m}\right)} \sum_{l=1}^{t} \sum_{i, j} \omega_{I, l} \lambda_{i j}^{l}\left[\omega_{i}\left|\omega_{j}\right| \omega_{i_{1}}|\ldots| \omega_{i_{m}}\right] .
$$

$4^{\text {e }}$ SÉRIE - TOME $42-2009-\mathrm{N}^{\mathrm{o}} 3$ 
This is integrable: it maps to 0 under $\wedge_{k}$ for $k \geq 2$, because $f$ is integrable, and it maps to 0 under $\wedge_{1}$ because of the quadratic relations (9.1). It follows that every element $f \in A(F)$ of weight $\geq 1$ such that $d f=0$ has a primitive. It is easy to write down a primitive of any element of weight 0 . Thus $H^{i}(A(F))=0$ for all $i \geq 1$, which completes the proof of the theorem.

\section{Index of notations}

We list the most frequently used notations, along with the section in which they are first defined. The integers $n=\ell+3$, where $\ell \geq 0$, are fixed.

\section{Section 2}

$\delta \quad$ A dihedral structure on a set $S$ with $n$ elements.

$\left(\mathbb{P}^{1}\right)_{*}^{n} \quad$ The set of $n$ distinct points in the projective line.

$\mathfrak{M}_{0, S} \quad$ The moduli space of curves of genus 0 with points marked by $S$.

$\mathfrak{M}_{0, S}^{\delta} \quad$ The dihedral extension of $\mathfrak{M}_{0, S}$.

$\overline{\mathfrak{M}}_{0, S} \quad$ The full compactification of $\mathfrak{M}_{0, S}$.

$\mathcal{O}\left(\mathfrak{M}_{0, S}\right) \quad$ The ring of regular functions on $\mathfrak{M}_{0, S}$.

$\chi_{S, \delta} \quad$ The set of all chords in the $n$-gon $(S, \delta)$.

$\chi_{S, \delta}^{k} \quad$ The set of all partial $k$-triangulations of $(S, \delta)$.

$\{i, j\} \sim_{\times}\{k, l\}$ The chords $\{i, j\},\{k, l\} \in \chi_{S, \delta}$ cross.

$\left\{u_{i j}:\{i, j\} \in \chi_{S, \delta}\right\}$ The set of dihedral coordinates on $\mathfrak{M}_{0, S}$.

$\left(x_{1}, \ldots, x_{\ell}\right) \quad$ The set of cubical coordinates on $\mathfrak{M}_{0, S}$.

$\left(t_{1}, \ldots, t_{\ell}\right) \quad$ The set of simplicial coordinates on $\mathfrak{M}_{0, S}$.

$\left(x_{1}^{\alpha}, \ldots, x_{\ell}^{\alpha}\right)$ The set of vertex coordinates corresponding to $\alpha \in \chi_{S, \delta}^{\ell}$.

$X_{S, \delta} \quad$ The open associahedron $X_{S, \delta} \subset \mathfrak{M}_{0, S}(\mathbb{R})$.

$\bar{X}_{S, \delta} \quad$ The closed associahedron $\bar{X}_{S, \delta} \subset \mathfrak{M}_{0, S}^{\delta}(\mathbb{R})$.

$F_{i j} \quad$ The face of $\bar{X}_{S, \delta}$ corresponding to the chord $\{i, j\} \in \chi_{S, \delta}$.

$F_{\alpha} \quad$ The intersection of faces $\bigcap_{\{i, j\} \in \alpha} F_{i j}$ corresponding to $\alpha \in \chi_{S, \delta}^{k}$.

$f_{T} \quad$ The forgetful map $f_{T}: \mathfrak{M}_{0, S} \rightarrow \mathfrak{M}_{0, T}$.

$m_{\square} \quad$ The cubical multiplication map.

$m_{\triangle} \quad$ The simplicial multiplication map.

\section{Section 3}

$A \quad$ An alphabet.

$\mathbb{Z}\langle A\rangle \quad$ The free tensor algebra on $A$.

ш The shuffle product.

$\Delta \quad$ The coproduct on the shuffle algebra.

$\varepsilon \quad$ The counit on the shuffle algebra.

$\partial_{a} \quad$ The operator acting by truncation on the left.

$M=\mathbb{A}^{\ell} \backslash \cup_{i=1}^{N} H_{i}$ The complement of an affine hyperplane arrangement.

$\mathcal{O}_{M} \quad$ The ring of regular functions on $M$.

$B\left(\mathcal{O}_{M}\right)=B(M)$ The reduced bar construction on $M$.

$B_{\mathcal{O}_{M^{\prime}}}(E) \quad$ The relative bar construction on $E$ with coefficients in $\mathcal{O}_{M^{\prime}}$.

$k\left\{\epsilon_{1}, \ldots, \epsilon_{\ell}\right\}$ The differential $k$-algebra of Laurent series in $\epsilon_{1}, \ldots, \epsilon_{\ell}$.

$U\left\{\epsilon_{1}, \ldots, \epsilon_{\ell}\right\}$ The differential $k$-algebra of logarithmic Laurent series.

$\mathfrak{u p}(R, \varepsilon) \quad$ The category of unipotent pointed extensions of $(R, \varepsilon)$. 


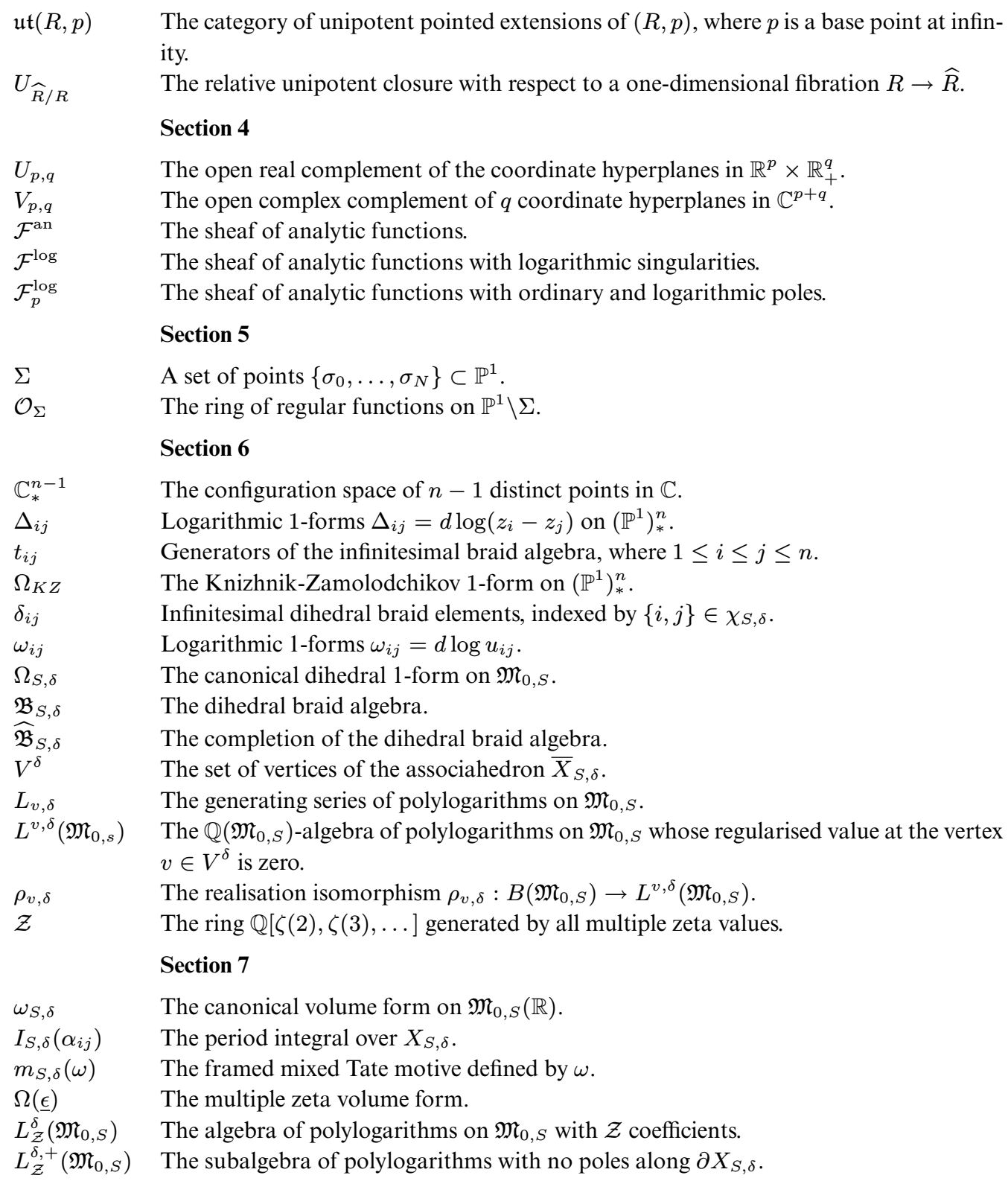

\section{REFERENCES}

[1] K. Аомото, Fonctions hyperlogarithmiques et groupes de monodromie unipotents, $J$. Fac. Sci. Univ. Tokyo Sect. IA Math. 25 (1978), 149-156.

[2] K. Аомото, Addition theorem of Abel type for hyper-logarithms, Nagoya Math. J. 88 (1982), 55-71.

[3] К. Аомото, Special values of hyperlogarithms and linear difference schemes, Illinois J. Math. 34 (1990), 191-216. 
[4] V. I. Arnol'D, The cohomology ring of the group of dyed braids, Mat. Zametki 5 (1969), 227-231.

[5] A. A. Beilinson, A. B. Goncharov, V. V. Schechtman, A. N. Varchenko, Aomoto dilogarithms, mixed Hodge structures and motivic cohomology of a pair of triangles in the plane, in Grothendieck Festschrift, 86, Birkhäuser, 1990, 135-171.

[6] A. Borel, J-P. Serre, Corners and arithmetic groups, Comment. Math. Helv. 48 (1973), 436-491.

[7] F. C. S. Brown, Polylogarithmes multiples uniformes en une variable, C. R. Math. Acad. Sci. Paris 338 (2004), 527-532.

[8] F. C. S. Brown, Single-valued hyperlogarithms and unipotent differential equations, preprint.

[9] K. T. Chen, Extension of $C^{\infty}$ function algebra by integrals and Malcev completion of $\pi_{1}$, Advances in Math. 23 (1977), 181-210.

[10] K. T. Chen, Iterated path integrals, Bull. Amer. Math. Soc. 83 (1977), 831-879.

[11] P. Deligne, Équations différentielles à points singuliers réguliers, Lecture Notes in Math., Vol. 163, Springer, 1970.

[12] P. Deligne, Théorie de Hodge. III, Publ. Math. I.H.É.S. 44 (1974), 5-77.

[13] P. Deligne, Le groupe fondamental de la droite projective moins trois points, in Galois groups over Q (Berkeley, CA, 1987), Math. Sci. Res. Inst. Publ. 16, Springer, 1989, 79-297.

[14] P. Deligne, A. B. Goncharov, Groupes fondamentaux motiviques de Tate mixte, Ann. Sci. École Norm. Sup. 38 (2005), 1-56.

[15] S. L. Devadoss, Tessellations of moduli spaces and the mosaic operad, in Homotopy invariant algebraic structures (Baltimore, MD, 1998), Contemp. Math. 239, Amer. Math. Soc., 1999, 91-114.

[16] S. L. Devadoss, Combinatorial equivalence of real moduli spaces, Notices Amer. Math. Soc. 51 (2004), 620-628.

[17] A. C. Dixon, On a certain double integral, Proc. London Math. Soc. 2 (1905), 8-15.

[18] V. G. Drinfel'D, On quasitriangular quasi-Hopf algebras and on a group that is closely connected with Gal $(\overline{\mathbf{Q}} / \mathbf{Q})$, Leningrad Math. Journal 2 (1991), 829-860.

[19] J. ÉCALLE, Singularités non abordables par la géométrie, Ann. Inst. Fourier (Grenoble) 42 (1992), 73-164.

[20] M. FALK, R. Randell, The lower central series of a fiber-type arrangement, Invent. Math. 82 (1985), 77-88.

[21] S. Fischler, Groupes de Rhin-Viola et intégrales multiples, J. Théor. Nombres Bordeaux 15 (2003), 479-534.

[22] E. Getzler, Operads and moduli spaces of genus 0 Riemann surfaces, in The moduli space of curves (Texel Island, 1994), Progr. Math. 129, Birkhäuser, 1995, 199-230.

[23] A. B. Goncharov, The dihedral Lie algebras and Galois symmetries of $\pi_{1}^{(l)}\left(\mathbb{P}^{1}-\right.$ $\left.\left(\{0, \infty\} \cup \mu_{N}\right)\right)$, Duke Math. J. 110 (2001), 397-487.

[24] A. B. Goncharov, Multiple $\zeta$-values, Galois groups, and geometry of modular varieties, in European Congress of Mathematics, Vol. I (Barcelona, 2000), Progr. Math. 201, Birkhäuser, 2001, 361-392. 
[25] A. B. Goncharov, Multiple polylogarithms and mixed Tate motives, preprint arXiv:math.AG/0103059.

[26] A. B. Goncharov, Periods and mixed motives, preprint arXiv:math.AG/0202154.

[27] A. B. Goncharov, Y. I. Manin, Multiple $\zeta$-motives and moduli spaces $\overline{\mathscr{M}}_{0, n}$, Compos. Math. 140 (2004), 1-14.

[28] J. Gonzalez-Lorca, Série de Drinfel'd, monodromie et algèbres de Hecke, Thèse de doctorat, École Normale Supérieure, 1998.

[29] P. GRIfFiths, W. SCHMid, Recent developments in Hodge theory: a discussion of techniques and results, in Discrete subgroups of Lie groups and applicatons to moduli (Internat. Colloq., Bombay, 1973), Oxford Univ. Press, 1975, 31-127.

[30] A. Grothendieck, On the de Rham cohomology of algebraic varieties, Publ. Math. I.H.É.S. 29 (1966), 95-103.

[31] R. M. Hain, The geometry of the mixed Hodge structure on the fundamental group, in Algebraic geometry, Bowdoin, 1985 (Brunswick, Maine, 1985), Proc. Sympos. Pure Math. 46, Amer. Math. Soc., 1987, 247-282.

[32] R. M. Hain, Classical polylogarithms, in Motives (Seattle, WA, 1991), Proc. Sympos. Pure Math. 55, Amer. Math. Soc., 1994, 3-42.

[33] R. M. Hain, R. MacPherson, Higher logarithms, Illinois J. Math. 34 (1990), 392475.

[34] M. Hoang Ngoc, M. Petitot, J. Van Den Hoeven, Shuffle algebra and polylogarithms, in Formal Power Series and Algebraic Combinatorics 1998, Toronto.

[35] M. E. Hoffman, Quasi-shuffle products, J. Algebraic Combin. 11 (2000), 49-68.

[36] M. M. Kapranov, The permutoassociahedron, Mac Lane's coherence theorem and asymptotic zones for the KZ equation, J. Pure Appl. Algebra 85 (1993), 119-142.

[37] V. G. Knizhnik, A. B. Zamolodchikov, Current algebra and Wess-Zumino model in two dimensions, Nuclear Phys. B 247 (1984), 83-103.

[38] T. Kohno, Série de Poincaré-Koszul associée aux groupes de tresses pures, Invent. Math. 82 (1985), 57-75.

[39] E. R. Kolchin, Differential algebra and algebraic groups, Academic Press, 1973, Pure and Applied Mathematics, Vol. 54.

[40] I. Lappo-DanilevskiI, Mémoires sur la théorie des systèmes des équations différentielles linéaires, Chelsea, New York, 1953.

[41] A. R. MAgID, Lectures on differential Galois theory, University Lecture Series 7, Amer. Math. Soc., 1994.

[42] L. Boutet de Monvel, Polylogarithmes, http://www.math.jussieu.fr/ boutet.

[43] P. Orlik, H. Terao, Arrangements of hyperplanes, Grund. Math. Wiss. 300, Springer, 1992.

[44] H. Poincaré, Sur les groupes d'équations linéaires, Acta Mathematica 4 (1884).

[45] G. Racinet, Doubles mélanges des polylogarithmes multiples aux racines de l'unité, Publ. Math. Inst. Hautes Études Sci. 95 (2002), 185-231.

[46] D. E. RADFORD, A natural ring basis for the shuffle algebra and an application to group schemes, J. Algebra 58 (1979), 432-454.

$4^{\text {e }}$ SÉRIE - TOME $42-2009-\mathrm{N}^{\mathrm{o}} 3$ 
[47] G. Rhin, C. Viola, On a permutation group related to $\zeta(2)$, Acta Arith. 77 (1996), 23-56.

[48] J. D. Stasheff, Homotopy associativity of $H$-spaces. I, Trans. Amer. Math. Soc. 108 (1963), 275-292.

[49] T. Terasoma, Selberg integrals and multiple zeta values, Compositio Math. 133 (2002), $1-24$.

[50] C. Voisin, Hodge theory and complex algebraic geometry. II, Cambridge Studies in Advanced Mathematics 77, Cambridge University Press, 2003.

[51] M. Waldschmidt, Valeurs zêta multiples. Une introduction, J. Théor. Nombres Bordeaux 12 (2000), 581-595.

[52] M. Yoshida, Fuchsian differential equations, Aspects of Mathematics, E11, Friedr. Vieweg \& Sohn, 1987.

[53] J. ZhaO, Multiple polylogarithms: analytic continuation, monodromy, and variations of mixed Hodge structures, in Contemporary trends in algebraic geometry and algebraic topology (Tianjin, 2000), Nankai Tracts Math. 5, World Sci. Publ., River Edge, NJ, 2002, 167-193.

[54] S. A. Zlobin, Integrals that can be represented as linear forms of generalized polylogarithms, Mat. Zametki [Math. Notes] 71 (2002), 782-787 [711-716].

[55] W. Zudilin, Well-poised hypergeometric transformations of Euler-type multiple integrals, J. London Math. Soc. 70 (2004), 215-230.

\author{
Francis C. S. BROWN \\ I.M.J. \& Université Paris VII \\ Département de Mathématiques \\ UMR 7586 du CNRS \\ Case 7012 \\ 2 place Jussieu \\ 75251 Paris Cedex 05, France \\ E-mail: brown@math.jussieu.fr
}

